\title{
COMPACTIFICATIONS OF SUBSCHEMES OF INTEGRAL MODELS OF SHIMURA VARIETIES
}

\author{
KAI-WEN LAN ${ }^{1}$ and BENOÎT STROH ${ }^{2}$ \\ ${ }^{1}$ University of Minnesota, Minneapolis, MN 55455, USA; \\ email: kwlan@math.umn.edu \\ ${ }^{2}$ C.N.R.S. and Institut de Mathématiques de Jussieu-Paris Rive Gauche, \\ 75252 Paris Cedex 05, France; \\ email: benoit.stroh@imj-prg.fr
}

Received 23 October 2016; accepted 13 July 2018

\begin{abstract}
We study several kinds of subschemes of mixed characteristic models of Shimura varieties which admit good (partial) toroidal and minimal compactifications, with familiar boundary stratifications and formal local structures, as if they were Shimura varieties in characteristic zero. We also generalize Koecher's principle and the relative vanishing of subcanonical extensions for coherent sheaves, and Pink's and Morel's formulas for étale sheaves, to the context of such subschemes.
\end{abstract}

2010 Mathematics Subject Classification: 11G18 (primary); 11G15, $11 \mathrm{~F} 75$ (secondary)

\section{Introduction}

Integral models of Shimura varieties and their compactifications have played crucially important roles in many recent developments in algebraic number theory. In most of these developments, it is desirable to have certain decompositions of their special fibers into disjoint unions of locally closed subsets, which allowed mathematicians to reduce or relate their questions to some simpler building blocks.

For example, in PEL-type cases without factors of type D, we have the $p$-rank strata and Newton strata for all models, the Oort central leaves and EkedahlOort strata for models above hyperspecial levels at $p$, and the Kottwitz-Rapoport strata for models above parahoric levels at $p$, on the reductions modulo $p$ of all integral models we consider. When the level and ramification at $p$ are mild, these

(C) The Author(s) 2018. This is an Open Access article, distributed under the terms of the Creative Commons Attribution-NonCommercialNoDerivatives licence (http://creativecommons.org/licenses/by-nc-nd/4.0/), which permits noncommercial re-use, distribution, and reproduction in any medium, provided the original work is unaltered and is properly cited. The written permission of Cambridge University Press must be obtained for commercial re-use or in order to create a derivative work. 
are known to form stratifications in the precise sense that closures of strata are again unions of strata. In general, they still form stratifications in a weaker sense that suitable unions form closed subsets, which are sufficiently useful for many applications.

On the other hand, it is also desirable to have nice total or partial compactifications of the integral models. For a long time, it was mainly the good reduction integral models or some parahoric variants of them which had been considered at all (see the introductions of [36, 78], and [79]). Nevertheless, in recent works by Madapusi Pera (see [50]) and us (with more elementary arguments; see [38, 41], and [43], and also [1] and [70]), a general principle has emerged-the difficulties in the construction of compactifications and in the construction of normal integral models with nice local properties are essentially disjoint from each other.

Roughly speaking, the first goal of this article is to uniformly construct good partial toroidal and minimal compactifications for many nice locally closed subsets or subschemes, without any detailed knowledge of their local properties, but with a long list of nice properties as if they were Shimura varieties in characteristic zero.

We have several motivations for this.

Firstly, while preparing our previous article [45], we observed that the supports of nearby cycles over the integral models of PEL-type or Hodge-type Shimura varieties we consider, even in the trivial coefficient case, enjoy some intriguing nice features near the toroidal and minimal boundary, which make it possible to talk about good toroidal and minimal compactifications of such supports. (We emphasize that in PEL-type cases we do allow arbitrarily high levels at $p$, for which no theory of local models is currently available.) Based on earlier experience of one of us (see $[\mathbf{8 0}, \mathbf{8 2}]$ ), we soon realized that the same can be said for several other kinds of subsets or subschemes of the integral models we consider, including the above-mentioned $p$-rank strata, Newton strata, Oort central leaves, Ekedahl-Oort strata, and Kottwitz-Rapoport strata (at least in PEL-type cases).

Secondly, in Boxer's recent work [12] on generalized Hasse invariants on Ekedahl-Oort strata, he introduced the notion of well-positioned subschemes near the boundaries of toroidal and minimal compactifications of the good reduction integral models constructed in [36], and used it to show that the Ekedahl-Oort strata extend to affine subschemes of the minimal compactifications. We observed that a slightly weaker notion than Boxer's naturally generalizes to cover many other interesting situations, including everything we mentioned in the previous paragraph. We shall call them well-positioned subsets or subschemes, from now on. 
Thirdly, we can generalize many useful results concerning the coherent and étale cohomology of integral models of Shimura varieties to the context of well-positioned subsets or subschemes - these can be considered the second goal of this article. They not only have many potential applications, but also clarify what were really needed in their original proofs. For example, for coherent cohomology, we can generalize Koecher's principle for the global sections of canonical extensions of automorphic bundles over partial toroidal compactifications, and the vanishing of higher direct images of subcanonical extensions of automorphic bundles under the canonical morphisms from the partial toroidal compactifications to the partial minimal compactifications, even when they are far from normal. For étale cohomology, under some technical assumptions, we can generalize Pink's formula (see [72]) for the pullbacks to boundary strata of the derived direct images of automorphic étale sheaves, under the canonical morphisms from Shimura varieties to the minimal compactifications; and also Morel's formula (see [58, 59, 61]) for the analogues for middle perversity extensions instead of derived direct images.

Here is an outline of this article.

In Section 2, we introduce and study the notions of well-positioned subsets and subschemes of the integral models of PEL-type or Hodge-type Shimura varieties we consider, and of their partial toroidal and minimal compactifications. In Section 2.1, we review the integral models we consider, and give a qualitative description of their toroidal and minimal compactifications. In Section 2.2, we introduce the well-positioned subsets and subschemes, and prove some basic lemmas. In Section 2.3, we construct the partial toroidal and minimal compactifications for all well-positioned subsets and subschemes, and include some basic consequences. For example, we show that the local properties of the partial toroidal compactifications are as nice as the given well-positioned subset (with its reduced subscheme structure) or subscheme. In Section 2.4, we show that the consideration of well-positioned subsets and subschemes, and their partial compactifications, are functorial in nature and compatible with Hecke actions. In Section 2.5, we present the generalizations of Koecher's principle and the vanishing of higher direct images alluded above.

In Section 3, we study many examples of well-positioned subsets and subschemes. We start with the seemingly trivial examples of pullbacks and fibers in Section 3.1. Then we proceed with the more interesting examples of the $p$-rank strata in Section 3.2, the Newton strata in Section 3.3, the Oort central leaves in Section 3.4, the Ekedahl-Oort strata in Section 3.5, and the KottwitzRapoport strata in Section 3.6, all in PEL-type cases (and often without factors of type D), by first introducing them as locally closed subsets, and then showing that they are well positioned as in Section 2.2, and hence admit partial toroidal 
and minimal compactifications as in Section 2.3. We have chosen to present these examples in PEL-type cases, often without factors of type D, because the theories are most complete and well understood in these cases. (Nevertheless, see $[48,85]$, for example, for some recent developments for Hodge-type Shimura varieties with hyperspecial levels at $p$.) The definitions we have used are not necessarily the ones of the greatest elegance, novelty, or historical importance, but rather ones that are most amenable to the consideration of semi-abelian degenerations near the boundary. (We apologize to any experts whose works we might have failed to highlight or even mention.) In Section 3.7, we study the supports of nearby cycles, and show that they are well positioned, under an assumption that is satisfied at a cofinal system of levels. The upshot is that, with little knowledge beyond the definitions, we can show that many subsets or subschemes are well positioned. Then they automatically admit partial toroidal and minimal compactifications with familiar properties, just like their ambient integral models of Shimura varieties, and they enjoy all the nice features we have abstractly established in Section 2.

In Section 4, we introduce and study the notion of well-positioned étale sheaves and complexes over the partial toroidal compactifications of the wellpositioned subsets introduced in Section 2. In Sections 4.1 and 4.2, we give their definitions and study their general properties, together with some examples. In Sections 4.3, 4.4, and 4.5, we present the generalizations of Pink's and Morel's formulas alluded above, and also a variant of Mantovan's formula with boundary terms (different from our previous generalization in [45]).

We emphasize that our results apply even when we have essentially no knowledge of the well-positioned subsets or subschemes being considered. For example, consider any good reduction $p$-integral model introduced by Kottwitz in [32, Section 5] in PEL-type cases not of type D, consider the intersection of a Newton and an Ekedahl-Oort strata on its characteristic $p$ special fiber, and consider just one irreducible component of its pullback to principal level $p^{2018}$, which we denote by $\mathrm{Y}$. We know essentially nothing about $\mathrm{Y}$, but we can still show that it is well positioned, that the (generally infinite-dimensional) coherent cohomology of its partial toroidal compactification $Y_{\Sigma}^{\text {tor }}$ still satisfies our generalization of Koecher's principle, and that the intersection complex of $Y_{\Sigma}^{\text {tor }}$ is well positioned and satisfies our generalization of Morel's formula over $Y^{\min }$.

We shall follow [36, Notation and Conventions] unless otherwise specified. While for practical reasons we cannot explain everything we need from the various constructions of toroidal and minimal compactifications we need, we recommend the reader to make use of the reasonably detailed index and table of contents there, when looking for the numerous definitions. For references to [36], the reader should also consult the errata available on the author's website for 
corrections to some known errors and imprecisions. We sometimes use materials in [45] without thoroughly reviewing them.

\section{Well-positioned subsets and subschemes of good integral models}

2.1. Background setting. Let $p>0$ be a rational prime number. Let us repeat [45, Assumption 2.1] as follows:

ASSUMPTION 2.1.1. Let $\mathrm{X}_{\mathcal{H}} \rightarrow \mathrm{S}$ be a scheme over the spectrum of a discrete valuation ring $R_{0}$ of mixed characteristics $(0, p)$, which is the pullback of one the following integral models in the literature: (The various notations $\mathrm{S}_{0}, \overrightarrow{\mathrm{S}}_{0}$, and so forth below are those in the works we cited, which we freely use, but mostly only in proofs.)

(Sm) A smooth integral model

$$
\mathrm{M}_{\mathcal{H}^{\square}} \rightarrow \mathrm{S}_{0}=\operatorname{Spec}\left(\mathcal{O}_{F_{0},(\square)}\right)
$$

defined as a moduli of abelian schemes with PEL structures at a neat level $\mathcal{H}^{\square} \subset \mathrm{G}\left(\hat{\mathbb{Z}}^{\square}\right)$, as in [36, Chs 1, 2, and 7], with $p \in \square$ and $\mathcal{H}=\mathcal{H}^{\square} \times$ $\prod_{q \in \square} \mathrm{G}\left(\mathbb{Z}_{q}\right)$. (When $\square=\{p\}$, it is shown in [36, Proposition 1.4.3.4] that the definition in [36, Section 1.4.1] by isomorphism classes agrees with the one in [36, Section 1.4.2] by $\mathbb{Z}_{(p)}^{\times}$-isogeny classes, the latter being Kottwitz's definition in [32, Section 5].)

(Nm) A flat integral model

$$
\overrightarrow{\mathrm{M}}_{\mathcal{H}} \rightarrow \overrightarrow{\mathrm{S}}_{0}=\operatorname{Spec}\left(\mathcal{O}_{F_{0},(p)}\right)
$$

of a moduli $\mathrm{M}_{\mathcal{H}} \rightarrow \mathrm{S}_{0}=\operatorname{Spec}\left(F_{0}\right)$ at a neat level $\mathcal{H} \subset \mathrm{G}(\hat{\mathbb{Z}})$ (essentially the same as above, but with $\square=\emptyset$ ) defined by taking normalizations over certain auxiliary good reduction models as in [38, Section 6] (which allow bad reductions due to arbitrarily high levels, ramifications, polarization degrees, and collections of isogenies). (In this case, we also allow $F_{0}$ to be a finite extension of the reflex field, with $M_{\mathcal{H}}$ and others replaced with their pullbacks.) For simplicity, we shall assume that, in the choice of the collection $\left\{\left(g_{\mathrm{j}}, L_{\mathrm{j}},\langle\cdot, \cdot\rangle_{\mathrm{j}}\right)\right\}_{\mathrm{j} \in \mathrm{J}}$ in [38, Section 2], we have $g_{\mathrm{j}}=1$ for all $\mathrm{j} \in \mathrm{J}$ and $\left(L_{\mathrm{j}_{0}},\langle\cdot, \cdot\rangle_{\mathrm{j}_{0}}\right)=\left(p^{r_{0}} L, p^{-2 r_{0}}\langle\cdot, \cdot\rangle\right)$ for some $\mathrm{j}_{0} \in \mathrm{J}$ and some $r_{0} \in \mathbb{Z}$. (This simplifying assumption imposes no restriction on the integral models we consider.) 
(Spl) A flat integral model

$$
\overrightarrow{\mathrm{M}}_{\mathcal{H}}^{\mathrm{spl}} \rightarrow \operatorname{Spec}\left(\mathcal{O}_{K}\right)
$$

of $\mathrm{M}_{\mathcal{H}} \otimes_{F_{0}} K \rightarrow \operatorname{Spec}(K)$ defined by taking normalizations as in [43, Section 2.4] over the splitting models defined by Pappas-Rapoport as in [68, Section 15]. (By taking normalizations, we mean we also allow $\mathcal{H}$ to be arbitrarily higher levels, not just the same levels considered in [68, Section 15].) For simplicity, we shall assume that, in the choice of the collection $\left\{\left(g_{\mathrm{j}}, L_{\mathrm{j}},\langle\cdot, \cdot\rangle_{\mathrm{j}}\right)\right\}_{\mathrm{j} \in \mathrm{J}}$ in [43, Choices 2.2.9], we have $g_{\mathrm{j}}=1$ for all $\mathrm{j} \in \mathrm{J}$ and $\left(L_{\mathrm{j}_{0}},\langle\cdot, \cdot\rangle_{\mathrm{j}_{0}}\right)=\left(p^{r_{0}} L, p^{-2 r_{0}}\langle\cdot, \cdot\rangle\right)$ for some $\mathrm{j}_{0} \in \mathrm{J}$ and some $r_{0} \in \mathbb{Z}$. (Again, this simplifying assumption imposes no restriction on the integral models we consider.)

(Hdg) A flat integral model

$$
\mathscr{S}_{K} \rightarrow \operatorname{Spec}\left(\mathscr{O}_{E,(v)}\right)
$$

in the notation of [50, Introduction] at a neat level $K$. For consistency with the notation in other cases, we shall denote $K, E$, and $\mathscr{S}_{K}$ as $\mathcal{H}, F_{0}$, and $\mathrm{M}_{\mathcal{H}}$, respectively, in what follows. Essentially by construction, there exists some auxiliary good reduction Siegel moduli $\mathrm{M}_{\mathcal{H}_{\text {aux }}} \rightarrow \operatorname{Spec}\left(\mathbb{Z}_{(p)}\right)$ in Case $(\mathrm{Sm})$ above, with a finite morphism $\mathrm{M}_{\mathcal{H}} \rightarrow \mathrm{M}_{\mathcal{H}_{\text {aux }}} \otimes_{\mathbb{Z}_{(p)}} \mathcal{O}_{F_{0},(v)}$ extending a closed immersion $\mathrm{M}_{\mathcal{H}} \otimes_{\mathbb{Z}} \mathbb{Q} \rightarrow \mathrm{M}_{\mathcal{H}_{\text {aux }}} \otimes_{\mathbb{Z}} F_{0}$.

In all cases, there is some group functor $\mathrm{G}$ over $\operatorname{Spec}(\mathbb{Z})$, and some reflex field $F_{0}$.

- In Cases $(\mathrm{Sm}),(\mathrm{Nm})$, and $(\mathrm{Spl})$, the integral models are defined by (among other data) an integral PEL datum $\left(\mathcal{O}, \star, L,\langle\cdot, \cdot\rangle, h_{0}\right)$ (cf. [36, Definition 1.2.1.3]), which defines the group functor $\mathrm{G}$ as in [36, Definition 1.2.1.6], and the reflex field $F_{0}$ as in [36, Definition 1.2.5.4]. For technical reasons, we shall insist that [36, Condition 1.4.3.10] is satisfied. Moreover, we shall assume as in [38, end of Section 2], [41, Theorem 6.1], and [43, Choices 2.2.10] that the image of $\mathcal{H} \subset \mathrm{G}(\hat{\mathbb{Z}})$ under the canonical homomorphism $\mathrm{G}(\hat{\mathbb{Z}}) \rightarrow \mathrm{G}\left(\hat{\mathbb{Z}}^{p}\right)$ is also neat. (In Case (Sm), this follows from the assumption that $\mathcal{H}^{\square} \subset \mathrm{G}\left(\hat{\mathbb{Z}}^{\square}\right)$ is neat.)

- In Case (Hdg), we still have an integral PEL datum defining the auxiliary good reduction Siegel moduli $\mathrm{M}_{\mathcal{H}_{\text {aux }}}$, which we abusively denote as $(\mathcal{O}, \star, L,\langle\cdot, \cdot\rangle$, $h_{0}$ ) (with $\mathcal{O}=\mathbb{Z}$, without 'aux' in the notation), which also defines a group functor $\mathrm{G}_{\text {aux }}$ with an injective homomorphism $\mathrm{G} \rightarrow \mathrm{G}_{\text {aux }}$. Moreover, we shall assume as in [50, Section 4.1.1] that $K=\mathcal{H}$ is of the form $\mathcal{H}=\mathcal{H}^{p} \mathcal{H}_{p}$ for some $\mathcal{H}^{p} \subset \mathrm{G}\left(\mathbb{A}^{\infty, p}\right)$ and $\mathcal{H}_{p} \subset \mathrm{G}\left(\mathbb{Q}_{p}\right)$, and that $\mathcal{H}^{p}$ is also neat. (The neatness of $\mathcal{H}^{p}$ is not explicitly emphasized in [50, Section 4.1.1], but is implicitly needed 
in the construction by normalizations in [50, Section 3.7.1], where $K=\mathcal{H}$ is assumed to be contained in some neat open compact $K^{\ddagger}=\mathcal{H}_{\text {aux }} \subset \mathrm{G}_{\text {aux }}\left(\mathbb{A}^{\infty}\right)$ of the form $\mathcal{H}_{\text {aux }}^{p} \mathcal{H}_{\text {aux }, p}$ with $\mathcal{H}_{\text {aux }}^{p} \subset \mathrm{G}_{\text {aux }}\left(\mathbb{A}^{\infty, p}\right)$ and $\mathcal{H}_{\text {aux }, p}=\mathrm{G}_{\text {aux }}\left(\mathbb{Z}_{p}\right) \subset$ $\mathrm{G}_{\text {aux }}\left(\mathbb{Q}_{p}\right)$. Since $\mathrm{G}_{\text {aux }}\left(\mathbb{Z}_{p}\right)$ is not neat, the neatness of $\mathcal{H}_{\text {aux }}$ forced $\mathcal{H}_{\text {aux }}^{p}$ and hence $\mathcal{H}^{p}$ to be also neat.)

Except in Case $(\mathrm{Sm})$, we allow the level $\mathcal{H} \subset \mathrm{G}\left(\mathbb{A}^{\infty}\right)$ to be arbitrarily high at $p$; that is, given any open compact subgroup of $G\left(\mathbb{Q}_{p}\right)$, there exists some $\mathcal{H}$ we allow whose image under the canonical homomorphism $\mathrm{G}\left(\mathbb{A}^{\infty}\right) \rightarrow \mathrm{G}\left(\mathbb{Q}_{p}\right)$ is smaller.

We shall say that we are in Case $(\mathrm{Sm}),(\mathrm{Nm}),(\mathrm{Spl})$, or $(\mathrm{Hdg})$ depending on the case in Assumption 2.1.1 from where $\mathrm{X}_{\mathcal{H}} \rightarrow \mathrm{S}$ is pulled back.

For each $X_{\mathcal{H}} \rightarrow S$ as in Assumption 2.1.1, we have good toroidal and minimal compactifications $\mathrm{X}_{\mathcal{H}, \Sigma}^{\text {tor }} \rightarrow \mathrm{S}$ and $\mathrm{X}_{\mathcal{H}}^{\text {min }} \rightarrow \mathrm{S}$, whose qualitative properties we summarized axiomatically in [45, Proposition 2.2], based on the constructions in $[36,38,41,43]$, and [50], which we also repeat as follows, for the sake of clarity:

Proposition 2.1.2. Let $\mathrm{X}_{\mathcal{H}} \rightarrow \mathrm{S}$ be as above. Then there is a canonical minimal compactification

$$
J_{\mathbf{X}_{\mathcal{H}}^{\min }}: \mathrm{X}_{\mathcal{H}} \hookrightarrow \mathrm{X}_{\mathcal{H}}^{\min }
$$

over $\mathrm{S}$, together with a canonical collection of toroidal compactifications

$$
J_{X_{\mathcal{H}, \Sigma}^{\text {tor }}}: \mathbf{X}_{\mathcal{H}} \hookrightarrow X_{\mathcal{H}, \Sigma}^{\text {tor }}
$$

over S, labeled by certain compatible collections $\Sigma$ of cone decompositions, satisfying the following properties:

(1) The structural morphism $\mathrm{X}_{\mathcal{H}}^{\min } \rightarrow \mathrm{S}$ is proper. For each $\Sigma$, there is a proper surjective structural morphism

$$
\oint_{\mathcal{H}, \Sigma}: X_{\mathcal{H}, \Sigma}^{\text {tor }} \rightarrow X_{\mathcal{H}}^{\min }
$$

which is compatible with $J_{\chi_{\mathcal{H}}^{\min }}$ and $J_{\chi_{\mathcal{H}, \Sigma}^{\mathrm{tor}}}$ in the sense that

$$
J_{\mathrm{X}_{\mathcal{H}}^{\min }}=\oint_{\mathcal{H}, \Sigma} \circ J_{\mathbf{X}_{\mathcal{H}, \Sigma}^{\mathrm{tor}}} .
$$

(2) The scheme $\mathrm{X}_{\mathcal{H}}^{\min }$ admits a stratification by locally closed subschemes $\mathbf{Z}$ flat over $\mathrm{S}$, each of which is isomorphic to an analogue of $\mathrm{X}_{\mathcal{H}}$ (in Cases $(\mathrm{Sm})$, $(\mathrm{Nm})$, or $(\mathrm{Spl})$ ) or a finite quotient of it (in Case (Hdg)). Moreover, the same incidence relation among strata holds on each fiber over $\mathrm{S}$. 
(3) Each $\Sigma$ is a set $\left\{\Sigma_{\mathrm{Z}}\right\}_{\mathrm{Z}}$ of cone decompositions $\Sigma_{\mathrm{Z}}$ with the same index set as that of the strata of $\mathrm{X}_{\mathcal{H}}^{\min }$-The elements of this index set can be called the cusp labels for $\mathrm{X}_{\mathcal{H}}$. For simplicity, we shall suppress such cusp labels and denote the associated objects with subscripts given by the strata $\mathrm{Z}$.

(4) For each stratum $\mathrm{Z}$, the cone decomposition $\Sigma_{\mathrm{Z}}$ is a cone decomposition of some $\mathbf{P}$, where $\mathbf{P}$ is the union of the interior $\mathbf{P}^{+}$of a homogeneous selfadjoint cone (see [4, Ch. 2]) and its rational boundary components, which is admissible with respect to some arithmetic group $\Gamma$ acting on $\mathbf{P}$ (and hence also on $\Sigma_{\mathrm{Z}}$ ). (For example, in the case of Siegel moduli, each $\mathbf{P}^{+}$can be identified with the space of $r \times r$ symmetric positive definite pairings for some integer $r$, and $\mathbf{P}$ can be identified with the space of $r \times r$ symmetric positive semidefinite pairings with rational radicals.) Then $\Sigma_{\mathrm{Z}}$ has a subset $\Sigma_{\mathrm{Z}}^{+}$forming a cone decomposition of $\mathbf{P}^{+}$. If $\tau$ is a cone in $\Sigma_{\mathrm{Z}}$ that is not in $\Sigma_{\mathbf{Z}}^{+}$, then there exist a stratum $\mathbf{Z}^{\prime}$ of $\mathbf{X}_{\mathcal{H}}^{\min }$, whose closure in $\mathbf{X}_{\mathcal{H}}^{\min }$ contains $\mathbf{Z}$, and a cone $\tau^{\prime}$ in $\Sigma_{Z^{\prime}}^{+}$, whose $\Gamma^{\prime}$-orbit is uniquely determined by the $\Gamma$-orbit of $\tau$ (where $\Gamma^{\prime}$ is the analogous arithmetic group acting on $\Sigma_{Z^{\prime}}$ ).

We may and we shall assume that $\Sigma$ is smooth and projective, and that, for each $\mathrm{Z}$ and $\sigma \in \Sigma_{\mathrm{Z}}^{+}$, its stabilizer $\Gamma_{\sigma}$ in $\Gamma$ is trivial.

(5) For each $\Sigma$, the associated $\mathrm{X}_{\mathcal{H}, \Sigma}^{\text {tor }}$ admits a stratification by locally closed subschemes $\mathrm{Z}_{[\sigma]}$ flat over $\mathrm{S}$, labeled by the strata $\mathrm{Z}$ of $\mathrm{X}_{\mathcal{H}}^{\min }$ and the orbits $[\sigma] \in \Sigma_{\mathbf{Z}}^{+} / \Gamma$. The stratifications of $\mathbf{X}_{\mathcal{H}, \Sigma}^{\text {tor }}$ and $\mathbf{X}_{\mathcal{H}}^{\min }$ are compatible with each other in a precise sense, which we summarize as follows: The preimage of a stratum $\mathbf{Z}$ of $\mathbf{X}_{\mathcal{H}}^{\min }$ is the (set-theoretic) disjoint union of the strata $\mathbf{Z}_{[\sigma]}$ of $\mathrm{X}_{\mathcal{H}, \Sigma}^{\text {tor }}$ with $[\sigma] \in \Sigma_{\mathrm{Z}}^{+} / \Gamma$. If $\tau$ is a face of a representative $\sigma$ of $[\sigma]$, which is identified (as in the property (4) above) with the $\Gamma^{\prime}$-orbit $\left[\tau^{\prime}\right]$ of some cone $\tau^{\prime}$ in $\Sigma_{Z^{\prime}}^{+}$, where $Z^{\prime}$ is a stratum whose closure in $\mathrm{X}_{\mathcal{H}}^{\min }$ contains $\mathrm{Z}$, then $\mathrm{Z}_{[\sigma]}$ is contained in the closure of $\mathrm{Z}_{\left[\tau^{\prime}\right]}$. The same incidence relation among strata holds on each fiber over $\mathrm{S}$.

(6) For each stratum $\mathrm{Z}$ of $\mathrm{X}_{\mathcal{H}}^{\min }$, there is a proper surjective morphism

$$
C \rightarrow \mathrm{Z}
$$

from a normal scheme which is flat over S, together with a morphism

$$
\Xi \rightarrow C
$$

of schemes which is a torsor under the pullback of a split torus $E$ with some character group $\mathbf{S}$ over $\operatorname{Spec}(\mathbb{Z})$, so that we have

$$
\Xi \cong \underline{\operatorname{Spec}}_{\mathscr{O}_{C}}\left(\bigoplus_{\ell \in \mathbf{S}} \Psi(\ell)\right)
$$


for some invertible sheaves $\Psi(\ell)$. (Each $\Psi(\ell)$ can be viewed as the subsheaf of $(\Xi \rightarrow C)_{*} \mathscr{O}_{\Xi}$ on which $E$ acts via the character $\ell \in \mathbf{S}$.) This character group $\mathbf{S}$ admits a canonical action of $\Gamma$, and its $\mathbb{R}$-dual $\mathbf{S}_{\mathbb{R}}^{\vee}:=\operatorname{Hom}_{\mathbb{Z}}(\mathbf{S}, \mathbb{R})$ canonically contains the above $\mathbf{P}$ as a subset with compatible $\Gamma$-actions.

(7) For each $\sigma \in \Sigma_{\mathrm{Z}}$, consider the canonical pairing $\langle\cdot, \cdot\rangle: \mathbf{S} \times \mathbf{S}_{\mathbb{R}}^{\vee} \rightarrow \mathbb{R}$ and

$$
\begin{aligned}
\sigma^{\vee} & :=\{\ell \in \mathbf{S}:\langle\ell, y\rangle \geqslant 0, \forall y \in \sigma\}, \\
\sigma_{0}^{\vee} & :=\{\ell \in \mathbf{S}:\langle\ell, y\rangle>0, \forall y \in \sigma\}, \\
\sigma^{\perp} & :=\{\ell \in \mathbf{S}:\langle\ell, y\rangle=0, \forall y \in \sigma\} \cong \sigma^{\vee} / \sigma_{0}^{\vee} .
\end{aligned}
$$

Then we have the affine toroidal embedding

$$
\Xi \hookrightarrow \Xi(\sigma):=\underline{\operatorname{Spec}}_{\mathscr{O}_{C}}\left(\bigoplus_{\ell \in \sigma^{\vee}} \Psi(\ell)\right) .
$$

The scheme $\Xi(\sigma)$ has a closed subscheme $\Xi_{\sigma}$ defined by the ideal sheaf corresponding to $\bigoplus_{\ell \in \sigma_{0}^{\vee}} \Psi(\ell)$, so that

$$
\Xi_{\sigma} \cong \underline{\operatorname{Spec}}_{\mathscr{O}_{C}}\left(\bigoplus_{\ell \in \sigma^{\perp}} \Psi(\ell)\right) .
$$

Then $\Xi(\sigma)$ admits a natural stratification by $\Xi_{\tau}$, where $\tau$ runs over all the faces of $\sigma$ in $\Sigma_{\mathrm{Z}}$.

(8) For each representative $\sigma \in \Sigma_{\mathrm{Z}}^{+}$of an orbit $[\sigma] \in \Sigma_{\mathrm{Z}}^{+} / \Gamma$, let

$$
\mathfrak{X}_{\sigma}:=(\Xi(\sigma))_{\Xi_{\sigma}}^{\wedge}
$$

denote the formal completion of $\Xi(\sigma)$ along $\Xi_{\sigma}$, and let $\left(\mathrm{X}_{\mathcal{H}, \Sigma}^{\mathrm{tor}}\right) \hat{\mathrm{Z}}_{[\sigma]}$ denote the formal completion of $\mathrm{X}_{\mathcal{H}, \Sigma}^{\text {tor }}$ along $\mathrm{Z}_{[\sigma]}$. Then there is a canonical isomorphism

$$
\mathfrak{X}_{\sigma} \cong\left(\mathbf{X}_{\mathcal{H}, \Sigma}^{\text {tor }}\right)_{\mathbf{Z}_{[\sigma]}}
$$

inducing a canonical isomorphism

$$
\Xi_{\sigma} \cong Z_{[\sigma]}
$$

(9) Let $x$ be a point of $\Xi_{\sigma}$, which can be canonically identified with a point of $Z_{[\sigma]}$ via the above isomorphism. Let us equip $\Xi(\sigma)$ with a coarser stratification induced by the $\Gamma$-orbits $[\tau]$ of $\tau$, where $\tau$ are the faces of $\sigma$. Each such orbit $[\tau]$ can be identified with the $\Gamma^{\prime}$-orbits $\left[\tau^{\prime}\right]$ of some cone $\tau^{\prime}$ 
in $\Sigma_{Z^{\prime}}^{+}$, where $Z^{\prime}$ is a stratum whose closure in $\mathbf{X}_{\mathcal{H}}^{\min }$ contains $\mathrm{Z}$. Then there exists an étale neighborhood

$$
\bar{U} \rightarrow \mathrm{X}_{\mathcal{H}, \Sigma}^{\mathrm{tor}}
$$

of $x$ and an étale morphism

$$
\bar{U} \rightarrow \Xi(\sigma)
$$

respecting $x$ such that the stratification of $\bar{U}$ induced by that of $\mathrm{X}_{\mathcal{H}, \Sigma}^{\text {tor }}$ coincides with the stratification of $\bar{U}$ induced by that of $\Xi(\sigma)$, in the sense that the preimage of the stratum $\mathrm{Z}_{\left[\tau^{\prime}\right]}$ of $\mathrm{X}_{\mathcal{H}, \Sigma}^{\text {tor }}$ coincides with the preimage of the $[\tau]$-stratum of $\Xi(\sigma)$ when $[\tau]$ determines $\left[\tau^{\prime}\right]$ as explained above; and such that the pullbacks of these étale morphisms to $Z_{[\sigma]}$ and to $\Xi_{\sigma}$ are both open immersions. (In particular, $\mathrm{X}_{\mathcal{H}, \Sigma}^{\text {tor }}$ and $\Xi(\sigma)$, equipped with their stratifications as explained above, are étale locally isomorphic at $x$.)

For our purpose in this article, it is useful to have the following more precise version of (8) of Proposition 2.1.2:

Proposition 2.1.3. Let us retain the same setting as in Proposition 2.1.2. For each given $\Sigma$, and for each $\mathrm{Z}$, consider the full toroidal embedding

$$
\bar{\Xi}_{\Sigma_{\mathrm{Z}}}=\bigcup_{\sigma \in \Sigma_{\mathrm{Z}}} \Xi(\sigma)
$$

defined by the cone decomposition $\Sigma_{\mathrm{Z}}$ (cf. [36, Theorem 6.1.2.8 and Section 6.2.5]), and consider the formal completion

$$
\mathfrak{X}_{\Sigma_{\mathrm{Z}}}:=\left(\bar{\Xi}_{\Sigma_{\mathrm{Z}}}\right)_{\bigcup_{\tau \in \Sigma_{\mathrm{Z}}^{+}}} \Xi_{\tau}
$$

of $\bar{\Xi}_{\Sigma_{\mathrm{Z}}}$ along its closed subscheme $\bigcup_{\tau \in \Sigma_{\mathrm{Z}}^{+}} \Xi_{\tau}$. Consider, for each $\sigma \in \Sigma_{\mathrm{Z}}^{+}$, the formal completion

$$
\mathfrak{X}_{\sigma}^{\circ}:=(\Xi(\sigma))_{\Xi(\sigma)^{+}}^{\wedge}
$$

of $\Xi(\sigma)$ along its closed subscheme

$$
\Xi(\sigma)^{+}:=\bigcup_{\tau \in \Sigma_{\mathrm{Z}}^{+}, \bar{\tau} \subset \bar{\sigma}} \Xi_{\tau} .
$$

Then $\mathfrak{X}_{\Sigma_{\mathrm{Z}}}$ admits an open covering by $\mathfrak{X}_{\sigma}^{\circ}$ for $\sigma$ running through elements of $\Sigma_{\mathrm{Z}}^{+}$, and we have canonical flat morphisms

$$
\mathfrak{X}_{\sigma} \rightarrow \mathfrak{X}_{\sigma}^{\circ} \hookrightarrow \mathfrak{X}_{\Sigma_{\mathrm{Z}}} \rightarrow \mathrm{X}_{\mathcal{H}, \Sigma}^{\text {tor }}
$$


(of locally ringed spaces) inducing isomorphisms

$$
\mathfrak{X}_{\sigma}^{\circ} \stackrel{\sim}{\rightarrow}\left(\mathbf{X}_{\mathcal{H}, \Sigma}^{\text {tor }}\right)_{\bigcup_{\tau \in \Sigma}^{+}, \bar{\tau} \subset \bar{\sigma}} \mathrm{Z}_{[\tau]}
$$

and

$$
\mathfrak{X}_{\Sigma \mathrm{Z}} / \Gamma \stackrel{\sim}{\rightarrow}\left(\mathrm{X}_{\mathcal{H}, \Sigma}^{\text {tor }}\right)_{\bigcup_{[\tau] \in \Sigma_{\mathrm{Z}}^{+} / \Gamma}} \mathrm{z}_{[\tau]}
$$

such that (2.1.6) induces (2.1.5) by restriction, extending the $\mathfrak{X}_{\sigma} \stackrel{\sim}{\rightarrow}\left(\mathbf{X}_{\mathcal{H}, \Sigma}^{\text {tor }}\right){\hat{Z_{[\sigma]}}}$ and $\Xi_{\sigma} \stackrel{\sim}{\rightarrow} \mathbf{Z}_{[\sigma]}$ in (8) of Proposition 2.1.2.

More precisely, for each $\sigma \in \Sigma_{Z}^{+}$, and for each affine open formal subscheme $\mathfrak{W}=\operatorname{Spf}(R)$ of $\mathfrak{X}_{\sigma}^{\circ}$, under the canonically induced (flat) morphisms

$$
W:=\operatorname{Spec}(R) \rightarrow \mathrm{X}_{\mathcal{H}, \Sigma}^{\text {tor }}
$$

and

$$
\operatorname{Spec}(R) \rightarrow \Xi(\sigma)
$$

induced by (2.1.5), the stratification of $W$ induced by that of $\mathrm{X}_{\mathcal{H}, \Sigma}^{\text {tor }}$ coincides with the stratification of $W$ induced by that of $\Xi(\sigma)$. In particular, the preimages of $\mathrm{X}_{\mathcal{H}}$ and $\Xi$ coincide as open subschemes of $W$. This open subscheme, which we denote as

$$
W^{0} \text {, }
$$

is the locus over $\operatorname{Spec}(R)$ where the pullback of any Mumford family is abelian. (For the meaning of Mumford families, see [36, Definition 6.2.5.28] in Case (Sm), and see [38, (8.29)] and [41, the proof of Lemma 4.13] in Case (Nm). In Case (Spl), the Mumford families are the pullbacks from those in Case (Nm); see [43, the proof of Lemma 3.2.22]. In Case (Hdg), we consider any pullbacks of Mumford families from auxiliary toroidal compactifications in Case (Sm).)

Proof. In Case (Sm), these follow from the very construction of Mumford families as relative schemes (with additional structures) over the formal boundary charts in [36, Section 6.2.5], and from the proof of [36, Theorem 6.4.1.1(5)] and its modification in the proof of [42, Lemma 1.3.2.41], based on [36, Theorem 6.4.1.1(6)], by matching the pullback of the tautological semi-abelian scheme

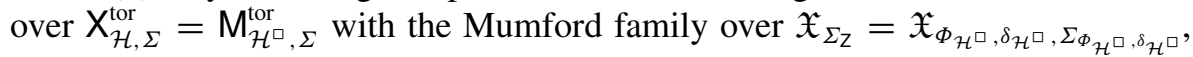
for each representative $\left(\Phi_{\mathcal{H}^{\square}}, \delta_{\mathcal{H}^{\square}}, \sigma\right)$ as in [36, Definition 6.2.6.1]. (Since the pullback of the stratification of $\mathrm{X}_{\mathcal{H}, \Sigma}^{\text {tor }}$ is determined by the theory of degeneration, it coincides with the pullback of the stratification of $\Xi(\sigma)$; see the proofs of [36, Propositions 6.3.1.6 and 6.3.2.10].) In Case ( $\mathrm{Nm})$, these follow from [41, Propositions 5.1 and 5.20]. In Case $(\mathrm{Spl})$, these follow from the proposition 
in Case $(\mathrm{Nm})$, because the stratification of $\overrightarrow{\mathrm{X}}_{\mathcal{H}, \Sigma}^{\mathrm{spl}, \text { tor }}$ is the pullback of that of $\overrightarrow{\mathrm{X}}_{\mathcal{H}, \Sigma}^{\text {tor }}$, and because $\vec{\Xi}_{\Phi_{\mathcal{H}}, \delta_{\mathcal{H}}}^{\text {spl }}(\sigma) \cong \vec{\Xi}_{\Phi_{\mathcal{H}}, \delta_{\mathcal{H}}}(\sigma) \times_{\vec{C}_{\Phi_{\mathcal{H}}, \delta_{\mathcal{H}}}} \vec{C}_{\Phi_{\mathcal{H}}, \delta_{\mathcal{H}}}^{\text {spl }}$ (see [43, (3.2.13)]). In Case ( $\mathrm{Hdg}$ ), this follows from the facts that $\mathrm{X}_{\mathcal{H}, \Sigma}^{\text {tor }}$ is the normalization (and hence finite) over some auxiliary good reduction toroidal compactification in Case $(\mathrm{Sm})$, and that each stratum $Z_{[\sigma]}$ of $X_{\mathcal{H}, \Sigma}^{\text {tor }}$ is open and closed in the preimage of a stratum of this auxiliary toroidal compactification, by the proof of [50, Proposition 4.2.13].

Let us also record the following strengthening of (9) of Proposition 2.1.2, which follows from the same argument of the proofs of [45, Proposition 2.2(9) and Corollary 2.4], but with the input [45, Proposition 2.2(8)] there replaced with Proposition 2.1.3 here:

Corollary 2.1.7 (Cf. [45, Corollary 2.4]). Let $x$ be any point of $\mathrm{X}_{\mathcal{H}, \Sigma}^{\text {tor }}$, which we may assume to lie on some stratum $\mathrm{Z}_{[\sigma]}$ above some stratum $\mathrm{Z}$ of $\mathrm{X}_{\mathcal{H}}^{\text {min }}$. Let $\sigma$ be any representative of $[\sigma]$, and let $E \hookrightarrow E(\sigma)$ and $E_{\sigma}$ be the affine toroidal embedding and the closed $\sigma$-stratum of $E(\sigma)$ over $\operatorname{Spec}(\mathbb{Z})$ (defined analogously as in the case of $\Xi \hookrightarrow \Xi(\sigma)$ and $\Xi_{\sigma}$, but simpler $)$. Let

$$
\tilde{Z}:=\bigcup_{[\tau] \in \Sigma_{\mathrm{Z}}^{+} / \Gamma} Z_{[\tau]}
$$

(with its reduced subscheme structure) in $\mathrm{X}_{\mathcal{H}, \Sigma}^{\text {tor }}$, which is the reduced subscheme of $\mathrm{X}_{\mathcal{H}, \Sigma}^{\text {tor }} \times_{\mathrm{X}_{\mathcal{H}}^{\min }}$ Z. Let

$$
E(\sigma)^{+}:=\bigcup_{\tau \in \Sigma_{\mathrm{Z}}^{+}, \bar{\tau} \subset \bar{\sigma}} E_{\tau}
$$

(with its reduced subscheme structure) in $E(\sigma)$. Then there exists an étale neighborhood

$$
\bar{U} \rightarrow \mathrm{X}_{\mathcal{H}, \Sigma}^{\text {tor }}
$$

of $x$ and an étale morphism

$$
\bar{U} \rightarrow E(\sigma) \times_{\operatorname{Spec}(\mathbb{Z})} C
$$

such that the stratifications of $\bar{U}$ induced by that of $\mathrm{X}_{\mathcal{H}, \Sigma}^{\text {tor }}$ and by that of $E(\sigma)$ coincide with each other; and such that the pullback of $\bar{U} \rightarrow \mathrm{X}_{\mathcal{H}, \Sigma}^{\text {tor }}$ under the canonical morphism $\tilde{Z} \rightarrow \mathrm{X}_{\mathcal{H}, \Sigma}^{\text {tor }}$ and the pullback of $\bar{U} \rightarrow E(\sigma) \times_{\operatorname{Spec}(\mathbb{Z})} C$ under the canonical morphism $E(\sigma)^{+} \times_{\operatorname{Spec}(\mathbb{Z})} C \rightarrow E(\sigma) \times_{\operatorname{Spec}(\mathbb{Z})} C$ are both open immersions. 
Suppose $\tau$ is a face of $\sigma$. Then the preimage of the stratum $\mathrm{Z}_{\left[\tau^{\prime}\right]}$ of $\mathrm{X}_{\mathcal{H}, \Sigma}^{\text {tor }}$ in $\bar{U}$, where $\left[\tau^{\prime}\right]$ is determined by $[\tau]$ as in (9) of Proposition 2.1.2, is the preimage of the stratum $E_{\tau}$ of $E(\sigma)$. If we denote by $\mathrm{Z}_{\left[\tau^{\prime}\right]}^{\text {tor }}$ the closure of $\mathrm{Z}_{\left[\tau^{\prime}\right]}$ in $\mathrm{X}_{\mathcal{H}, \Sigma}^{\text {tor }}$, and by $E_{\tau}(\sigma)$ the closure of $E_{\tau}$ in $E(\sigma)$, then the above implies that, étale locally at $x$, the open immersion

$$
J_{Z_{\left.\tau^{\prime}\right]}^{\text {tor }}}: Z_{\left[\tau^{\prime}\right]} \hookrightarrow Z_{\left[\tau^{\prime}\right]}^{\text {tor }}
$$

can be identified with the product of the canonical open immersion

$$
J_{E_{\tau}(\sigma)}: E_{\tau} \hookrightarrow E_{\tau}(\sigma)
$$

with the identity morphism on $C$.

In particular, when $\tau=\{0\}$, this means the preimage $U$ of $\mathrm{X}$ in $\bar{U}$ coincides with the preimage of $E$. Moreover, étale locally at $x$, the open immersion

$$
J_{X_{\mathcal{H}, \Sigma}^{\text {tor }}}: X \hookrightarrow X_{\mathcal{H}, \Sigma}^{\text {tor }}
$$

can be identified with the product of the canonical open immersion

$$
J_{E(\sigma)}: E \hookrightarrow E(\sigma)
$$

with the identity morphism on $C$.

In the remainder of this subsection, we record some special cases where $C \rightarrow \mathbf{Z}$ is known to be an abelian scheme torsor for each $Z$.

REMARK 2.1.8. Already in Case (Sm), where $\mathcal{H}^{\square} \subset \mathrm{G}\left(\hat{\mathbb{Z}}^{\square}\right)$ has no factor at $p$, the morphism $C \rightarrow \mathrm{Z}$ might not be an abelian scheme for each $\mathrm{Z}$, for an arbitrary $\mathcal{H}$ - see the errata for [36] on the author's website, and also the clarification in [42, Remark 1.3.1.6]. (It is only an abelian scheme torsor over a finite étale cover of Z.) Nevertheless, $C \rightarrow Z$ is indeed an abelian scheme for all $Z$ when $\mathcal{H}^{\square}$ is a principal level

$$
\mathcal{U}^{\square}(m):=\operatorname{ker}\left(\mathrm{G}\left(\hat{\mathbb{Z}}^{\square}\right) \rightarrow \mathrm{G}(\mathbb{Z} / m \mathbb{Z})\right)
$$

for some integer $m$ prime to $\square$, because the constructions in [36, Sections 6.2.26.2.3] remain valid, despite the mistake when taking quotients in [36, Section 6.2.4].

Consider the following special case of Case $(\mathrm{Nm})$ in Assumption 2.1.1: Suppose $p$ is a good prime (as in [36, Definition 1.4.1.1]) for an integral PEL datum $\left(\mathcal{O}, \star, L,\langle\cdot, \cdot\rangle, h_{0}\right)$ as in Assumption 2.1.1 (which we have insisted to satisfy [36, Condition 1.4.3.10]). Consider the trivial collection $\mathbf{J}=\left\{\mathrm{j}_{0}\right\}$ with $\left\{\left(g_{\mathrm{j}_{0}}, L_{\mathrm{j}_{0}},\langle\cdot, \cdot\rangle_{\mathrm{j}_{0}}\right)\right\}=\{(1, L,\langle\cdot, \cdot\rangle)\}$, as in [38, Example 2.3]. Let $\mathcal{H}$ be the 
principal level

$$
\mathcal{U}(n):=\operatorname{ker}(\mathrm{G}(\hat{\mathbb{Z}}) \rightarrow \mathrm{G}(\mathbb{Z} / n \mathbb{Z}))
$$

for some $n=n_{0} p^{r}$, where $n_{0} \geqslant 3$ is an integer prime to $p$, and where $r \geqslant 0$, so that

$$
\mathcal{H}^{p}=\mathcal{U}^{p}\left(n_{0}\right):=\operatorname{ker}\left(\mathrm{G}\left(\hat{\mathbb{Z}}^{p}\right) \rightarrow \mathrm{G}\left(\mathbb{Z} / n_{0} \mathbb{Z}\right)\right)
$$

is neat. Let

$$
\mathcal{H}_{0}:=\mathcal{U}\left(n_{0}\right):=\operatorname{ker}\left(\mathrm{G}(\hat{\mathbb{Z}}) \rightarrow \mathrm{G}\left(\mathbb{Z} / n_{0} \mathbb{Z}\right)\right)=\mathcal{H}^{p} \mathrm{G}\left(\mathbb{Z}_{p}\right) .
$$

Then $X_{\mathcal{H}} \rightarrow S$ (respectively $X_{\mathcal{H}_{0}} \rightarrow \mathrm{S}$ ) is a pullback of $\overrightarrow{\mathrm{M}}_{\mathcal{H}} \rightarrow \overrightarrow{\mathrm{S}}_{0}$ (respectively $\left.\overrightarrow{\mathrm{M}}_{\mathcal{H}_{0}} \rightarrow \overrightarrow{\mathrm{S}}_{0}\right)$ under some morphism $\mathrm{S} \rightarrow \overrightarrow{\mathrm{S}}_{0}=\operatorname{Spec}\left(\mathcal{O}_{F_{0},(p)}\right)$.

LEMMA 2.1.9. With the setting as above in Case (Nm), the morphism $C \rightarrow Z$ at level $\mathcal{H}$ is an abelian scheme for all $\mathrm{Z}$. Moreover, if we denote by $C_{0} \rightarrow \mathrm{Z}_{0}$ the analogous morphism at level $\mathcal{H}_{0}$, then the canonical morphism $C \rightarrow C_{0} \times \mathrm{Z}_{0} \mathbf{Z}$ can be identified with the multiplication by $p^{r}$ on the abelian scheme $C$ over $\mathrm{Z}$.

Proof. Let $\left(\mathrm{Z}_{\mathcal{H}}, \Phi_{\mathcal{H}}, \delta_{\mathcal{H}}\right)$ be a representative of cusp label for $\mathrm{M}_{\mathcal{H}}$. Let us denote by $\left(\mathrm{Z}_{\mathcal{H}^{p}}, \Phi_{\mathcal{H}^{p}}, \delta_{\mathcal{H}^{p}}\right)$ the prime-to- $p$ part of $\left(\mathrm{Z}_{\mathcal{H}}, \Phi_{\mathcal{H}}, \delta_{\mathcal{H}}\right)$, and by $\left(\mathrm{Z}_{\mathcal{H}_{0}}, \Phi_{\mathcal{H}_{0}}, \delta_{\mathcal{H}_{0}}\right)$ the induced representative at level $\mathcal{H}_{0}$. It suffices to show that, in the notation of [38, Section 8], $\vec{C}_{\Phi_{\mathcal{H}}, \delta_{\mathcal{H}}} \rightarrow \overrightarrow{\mathrm{M}}_{\mathcal{H}}^{Z_{\mathcal{H}}}$ is an abelian scheme, and the canonical morphism $\vec{C}_{\Phi_{\mathcal{H}}, \delta_{\mathcal{H}}} \rightarrow \vec{C}_{\Phi_{\mathcal{H}_{0}}, \delta_{\mathcal{H}_{0}}} \times_{\overrightarrow{\mathrm{M}}_{\mathcal{H}_{0}}^{Z_{\mathcal{H}}}} \overrightarrow{\mathrm{M}}_{\mathcal{H}}^{\mathcal{Z}_{\mathcal{H}}}$ can be identified with the multiplication by $p^{r}$ on the abelian scheme $\vec{C}_{\Phi_{\mathcal{H}}, \delta_{\mathcal{H}}}$ over $\overrightarrow{\mathrm{M}}_{\mathcal{H}}^{\mathrm{zH}}$.

Since $p$ is a good prime for $\left(\mathcal{O}, \star, L,\langle\cdot, \cdot\rangle, h_{0}\right)$, we have the morphisms

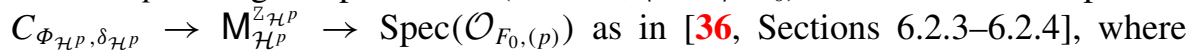
the first one is an abelian scheme. By [36, Proposition 1.4.4.3], the canonical morphism $\mathbf{M}_{\mathcal{H}_{0}}^{Z_{\mathcal{H}_{0}}} \rightarrow \mathbf{M}_{\mathcal{H}^{p}}^{Z_{\mathcal{H}}^{p}} \otimes_{\mathbb{Z}} \mathbb{Q}$ is an open and closed immersion. Since the schemes $\overrightarrow{\mathrm{M}}_{\mathcal{H}_{0}}^{\mathrm{H}_{\mathcal{H}}}$ and $\overrightarrow{\mathrm{M}}_{\mathcal{H}}^{\mathrm{Z} \mathcal{H}}$ over $\overrightarrow{\mathrm{S}}_{0}=\operatorname{Spec}\left(\mathcal{O}_{F_{0},(p)}\right)$ are independent of the auxiliary choices (see [38, Propositions 6.1 and 7.4]), by taking $\mathrm{M}_{\mathcal{H}^{p}}^{\mathrm{H}^{p}}$ as an auxiliary good reduction model, we have an open and closed immersion $\overrightarrow{\mathrm{M}}_{\mathcal{H}_{0}}^{\mathrm{H}_{0}} \hookrightarrow \mathrm{M}_{\mathcal{H}^{p}}^{Z_{\mathcal{H}}}$, and we can take $\overrightarrow{\mathrm{M}}_{\mathcal{H}}^{Z_{\mathcal{H}}}$ to be the normalization of $\mathrm{M}_{\mathcal{H}^{p}}^{Z_{\mathcal{H}^{p}}}$ under the composition $\mathrm{M}_{\mathcal{H}}^{Z \mathcal{H}} \rightarrow \mathrm{M}_{\mathcal{H}_{0}}^{Z_{\mathcal{H}_{0}}} \rightarrow \overrightarrow{\mathrm{M}}_{\mathcal{H}_{0}}^{Z_{\mathcal{H}_{0}}} \hookrightarrow \mathrm{M}_{\mathcal{H}^{p}}^{Z_{\mathcal{H}}}$ of canonical morphisms.

Since $\mathcal{H}^{p}$ is a principal level, by the construction in [36, Section 6.2.3], $C_{\Phi_{\mathcal{H}}, \delta_{\mathcal{H}^{p}}} \rightarrow \mathrm{M}_{\mathcal{H}^{p}}^{z_{\mathcal{H}^{p}}}$ is an abelian scheme. By the same reasoning as in the previous paragraph, $\vec{C}_{\Phi_{\mathcal{H}_{0}}, \delta_{\mathcal{H}_{0}}} \rightarrow \overrightarrow{\mathrm{M}}_{\mathcal{H}_{0}}^{\mathrm{Z} \mathcal{H}_{0}}$ is canonically isomorphic to the pullback of $C_{\Phi_{\mathcal{H}^{p}, \delta_{\mathcal{H}}^{p}}} \rightarrow \mathrm{M}_{\mathcal{H}^{p}}^{Z_{\mathcal{H}^{p}}}$ under the open and closed immersion $\overrightarrow{\mathrm{M}}_{\mathcal{H}_{0}}^{\mathrm{Z}_{\mathcal{H}_{0}}} \hookrightarrow \mathrm{M}_{\mathcal{H}^{p}}^{Z_{\mathcal{H}^{p}}}$, which is also an abelian scheme. Since $\mathcal{H}=\mathcal{U}\left(n_{0} p^{r}\right)$ and $\mathcal{H}_{0}=\mathcal{U}\left(n_{0}\right)$ are both 
principal levels, the canonical morphism $C_{\Phi_{\mathcal{H}}, \delta \mathcal{H}} \rightarrow C_{\Phi_{\mathcal{H}_{0}}, \delta_{\mathcal{H}_{0}}} \times_{\mathrm{M}_{\mathcal{H}_{0}}}^{z_{\mathcal{H}_{0}}} \mathrm{M}_{\mathcal{H}}^{z_{\mathcal{H}}}$ can be identified with the multiplication by $p^{r}$ on the abelian scheme $C_{\Phi_{\mathcal{H}}, \delta_{\mathcal{H}}}$ over $\mathrm{M}_{\mathcal{H}}^{Z_{\mathcal{H}}}$. Hence, there is also an isomorphism $C_{\Phi_{\mathcal{H}}, \delta_{\mathcal{H}}} \stackrel{\sim}{\rightarrow} C_{\Phi_{\mathcal{H}_{0}}, \delta_{\mathcal{H}_{0}}} \times_{\mathrm{M}_{\mathcal{H}_{0}}^{z}}^{z_{\mathcal{H}_{0}}} \mathrm{M}_{\mathcal{H}}^{z_{\mathcal{H}}}$, which extends to an isomorphism $\vec{C}_{\Phi_{\mathcal{H}}, \delta_{\mathcal{H}}} \stackrel{\sim}{\rightarrow} \vec{C}_{\Phi_{\mathcal{H}_{0}}, \delta_{\mathcal{H}_{0}}} \times_{\overrightarrow{\mathrm{M}}_{\mathcal{H}_{0}}} \overrightarrow{\mathcal{H}}_{\mathcal{H}_{0}} \overrightarrow{\mathrm{M}}_{\mathcal{H}}^{Z_{\mathcal{H}}}$, by Zariski's main theorem, because abelian schemes are smooth, and because the base scheme $\overrightarrow{\mathrm{M}}_{\mathcal{H}}^{Z_{\mathcal{H}}}$ is noetherian normal by construction. The assertions in the first paragraph above now follow.

Lemma 2.1.10. In Case (Spl) that is based on the same setting as above in Case $(\mathrm{Nm})$ (see the paragraph preceding Lemma 2.1.9), the analogous statements in Lemma 2.1.9 also hold.

Proof. For each representative $\left(\mathrm{Z}_{\mathcal{H}}, \Phi_{\mathcal{H}}, \delta_{\mathcal{H}}\right)$ of cusp label for $\mathrm{M}_{\mathcal{H}}$, by [43, Definition 3.2.3], $\vec{C}_{\Phi_{\mathcal{H}}, \delta_{\mathcal{H}}}^{\mathrm{spl}}$ is the normalization of $\vec{C}_{\Phi_{\mathcal{H}}, \delta_{\mathcal{H}}} \times_{\overrightarrow{\mathrm{M}}_{\mathcal{H}}^{z_{\mathcal{H}}}} \overrightarrow{\mathrm{M}}_{\mathcal{H}}^{\mathrm{Z} \mathcal{H}, \text { spl }}$. When $\vec{C}_{\Phi_{\mathcal{H}}, \delta_{\mathcal{H}}} \rightarrow \overrightarrow{\mathrm{M}}_{\mathcal{H}}^{Z_{\mathcal{H}}}$ is an abelian scheme, this fiber product is already normal because $\overrightarrow{\mathrm{M}}_{\mathcal{H}}^{Z_{\mathcal{H}}, \text { spl }}$ is. Hence, $\vec{C}_{\Phi_{\mathcal{H}}, \delta_{\mathcal{H}}}^{\text {spl }} \rightarrow \overrightarrow{\mathrm{M}}_{\mathcal{H}}^{Z_{\mathcal{H}} \text {, spl }}$ is the pullback of $\vec{C}_{\Phi_{\mathcal{H}}, \delta_{\mathcal{H}}} \rightarrow \overrightarrow{\mathrm{M}}_{\mathcal{H}}^{z_{\mathcal{H}}}$, and the lemma follows.

REMARK 2.1.11. Other than the above special cases in Cases $(\mathrm{Nm})$ and $(\mathrm{Spl})$ (with the restrictive assumption that $\mathbf{J}=\left\{\mathrm{j}_{0}\right\}$, as in the paragraph preceding Lemma 2.1.9), it is also true in many other special cases that $C \rightarrow Z$ is an abelian scheme, or at least an abelian scheme torsor, for each Z. See, for example, the Siegel moduli with parahoric levels in [78]. (It is plausible that the argument there can be generalized to all cases in Cases $(\mathrm{Nm})$ and $(\mathrm{Spl})$ where $p$ is good for $\left(\mathcal{O}, \star, L,\langle\cdot, \cdot\rangle, h_{0}\right)$, and where $\mathcal{H}=\mathcal{H}^{p} \mathcal{H}_{p}$ for some principal $\mathcal{H}^{p} \subset \mathrm{G}\left(\hat{\mathbb{Z}}^{p}\right)$ and for some parahoric subgroup $\mathcal{H}_{p}$ of $\mathrm{G}\left(\mathbb{Q}_{p}\right)$ that is the identity component of the stabilizer of the base change of the collection $\mathscr{L}$ to $\mathbb{Z}_{p}$.)

REMARK 2.1.12. When this article was first written, our treatment in Case (Hdg) was based on the 2015 version of [50], and hence inherited its assumptions on $\mathcal{H}$ which ensure that the morphism $\mathrm{M}_{\mathcal{H}} \rightarrow \mathrm{M}_{\mathcal{H}_{\text {aux }}} \otimes_{\mathbb{Z}_{(p)}} \mathcal{O}_{F_{0},(v)}$ (in Assumption 2.1.1) is a closed immersion. This was also why we assumed in [45] and [46] that, in Case (Hdg) there, the level at $p$ was exactly the pullback of some hyperspecial level at $p$ of a symplectic similitude group (see, in particular, [46, Remark 2.1]). Henceforth, this assumption has been removed in the 2018 version of [50], so now we can also allow arbitrarily high levels at $p$ in Case (Hdg). We can similarly remove the corresponding assumptions in [45] and [46], because exactly the 
same arguments in [46] also work with the 2018 version of [50], except with the minor update that, in [46, proof of Proposition 2.4], the fifth sentence in the fifth paragraph should refer to [50, Definition 5.1.1] instead of 5.1.2.

2.2. Well-positioned subsets and subschemes. Let $\mathrm{T}$ be a locally noetherian scheme over $\mathrm{S}$. For the schemes such as $\mathrm{X}_{\mathcal{H}}$ (and morphisms among them) over $\mathrm{S}$, we shall denote their pullbacks to $\mathrm{T}$ by $\left(\mathrm{X}_{\mathcal{H}}\right)_{\mathrm{T}}$, and so forth.

DEFINITION 2.2.1. We say that a locally closed subset (respectively subscheme) $\mathrm{Y}$ of $\left(\mathrm{X}_{\mathcal{H}}\right)_{\mathrm{T}}$ is well positioned if there exists a collection

$$
Y^{\natural}=\left\{Y_{Z}^{\natural}\right\}_{Z}
$$

indexed by the strata $Z$ of $X_{\mathcal{H}}^{\min }$, where each $Y_{Z}^{\natural}$ is a locally closed subset (respectively subscheme) of $Z_{T}$ such that, for each $\mathfrak{W}$ as in Proposition 2.1.3, the pullback of $Y$ to $\left(W^{0}\right)_{\mathrm{T}}$ under the induced morphism $W^{0} \rightarrow \mathrm{X}_{\mathcal{H}}$ is, as a subset (respectively subscheme) of $\left(W^{0}\right)_{\mathrm{T}}$, the pullback of $Y_{Z}^{\natural}$ under the composition $W^{0} \rightarrow Z$ of the induced morphism $W^{0} \rightarrow \Xi$ with the canonical morphisms $\Xi \rightarrow C \rightarrow Z$. For convenience, for each $Y_{Z}^{\natural}$ as above, we shall also denote by $Y_{C}^{\natural}$ the pullback of $Y_{Z}^{\natural}$ under $C \rightarrow Z$, as a subset (respectively subscheme). We shall say that $Y^{\natural}$ is associated with $Y$.

LEMMA 2.2.2. In Definition 2.2.1, it suffices to verify the condition for some affine open covering of $\mathfrak{X}_{\Sigma_{\mathrm{Z}}}=\bigcup_{\sigma \in \Sigma_{\mathrm{Z}}^{+}} \mathfrak{X}_{\sigma}^{\circ}$ by affine formal schemes $\mathfrak{W}$ 's as in Proposition 2.1.3 for just one collection $\Sigma$ of cone decompositions.

Proof. Since locally closed subsets or subschemes coincide if and only if they do over the open subsets in an open covering, for each $\Sigma$, it suffices to verify the condition in Definition 2.2.1 for some open covering as in the statement of the lemma. Since every two $\Sigma$ 's has a common refinement, it suffices to show that, if $\Sigma^{\prime}$ is a refinement of $\Sigma$, then the condition holds for $\Sigma$ if and only if it holds for $\Sigma^{\prime}$. In this case, the canonical morphism

$$
\mathrm{X}_{\mathcal{H}, \Sigma^{\prime}}^{\text {tor }} \rightarrow \mathrm{X}_{\mathcal{H}, \Sigma}^{\text {tor }}
$$

is proper, under which $\bigcup_{\left[\sigma^{\prime}\right] \in \Sigma_{Z}^{\prime \prime} / \Gamma} Z_{\left[\sigma^{\prime}\right]}$ is the reduced subscheme of the preimage of $\bigcup_{[\sigma] \in \Sigma_{\mathrm{Z}}^{+} / \Gamma} Z_{[\sigma]}$, which induces a proper morphism

$$
\left(\mathbf{X}_{\mathcal{H}, \Sigma^{\prime}}^{\text {tor }}\right) \hat{\cup}_{\left[\sigma^{\prime}\right] \in \Sigma_{Z}^{\prime} / \Gamma} \mathrm{Z}_{\left[\sigma^{\prime}\right]} \rightarrow\left(\mathbf{X}_{\mathcal{H}, \Sigma}^{\text {tor }}\right) \hat{\bigcup}_{[\sigma] \in \Sigma_{\mathbf{Z}}^{+} / \Gamma} \mathrm{Z}_{[\sigma]}
$$


between the formal completions. On the other hand, for each $\sigma \in \Sigma_{\mathrm{Z}}^{+}$, consider

$$
\Xi(\sigma)^{\prime}:=\bigcup_{\tau \in \Sigma_{\bar{Z}}^{\prime+}, \tau \subset \sigma} \Xi(\tau),
$$

which admits a proper morphism

$$
\Xi(\sigma)^{\prime} \rightarrow \Xi(\sigma)
$$

extending the identity morphism on $\Xi$ and inducing a proper morphism

$$
\bigcup_{\tau \in \Sigma_{Z}^{\prime+}, \tau \subset \sigma} \mathfrak{X}_{\tau}^{\circ} \rightarrow \mathfrak{X}_{\sigma}^{\circ}
$$

compatible with the compositions of morphisms

$$
\mathfrak{X}_{\tau}^{\circ} \hookrightarrow \mathfrak{X}_{\Sigma_{\mathrm{Z}}^{\prime}} / \Gamma \stackrel{\sim}{\rightarrow}\left(\mathrm{X}_{\mathcal{H}, \Sigma^{\prime}}^{\text {tor }}\right)_{\bigcup_{\left[\sigma^{\prime}\right] \in \Sigma_{Z}^{\prime \prime} / \Gamma}} \mathrm{Z}_{\left[\sigma^{\prime}\right]}
$$

and

$$
\mathfrak{X}_{\sigma}^{\circ} \hookrightarrow \mathfrak{X}_{\Sigma_{\mathrm{Z}}} / \Gamma \stackrel{\sim}{\rightarrow}\left(\mathrm{X}_{\mathcal{H}, \Sigma}^{\text {tor }}\right)_{\bigcup_{[\sigma] \in \Sigma_{\mathrm{Z}} / \Gamma}} \mathrm{Z}_{[\sigma]},
$$

where the first morphisms in the compositions are open immersions, and where the second morphisms in the compositions are the canonical isomorphisms, as in Proposition 2.1.3. Therefore, for each affine open formal subscheme $\mathfrak{W}=\operatorname{Spf}(R)$ of $\mathfrak{X}_{\sigma}^{\circ}$, which induces a canonical morphism $W=\operatorname{Spec}(R) \rightarrow \Xi(\sigma)$, the pullback of $W$ under $\Xi(\sigma)^{\prime} \rightarrow \Xi(\sigma)$ is covered by finitely many $W_{i}=\operatorname{Spec}\left(R_{i}\right)$, where $W_{i} \rightarrow \Xi(\sigma)^{\prime}$ is induced by some affine open formal subscheme $\mathfrak{W}_{i}=\operatorname{Spf}\left(R_{i}\right)$ of $\mathfrak{X}_{\tau_{i}}^{\circ}$, for some $\tau_{i} \in \Sigma_{Z}^{\prime,+}$ such that $\tau_{i} \subset \sigma$. Since $\Xi(\sigma)^{\prime} \rightarrow \Xi(\sigma)$ induces the identity morphism on $\Xi$ by pullback on the target, $W^{0}=\bigcup_{i} W_{i}^{0}$ is an open covering, and the lemma follows, as desired.

The following three lemmas follow immediately from the definitions:

LEMMA 2.2.3. Suppose a locally closed subset (respectively subscheme) $\mathrm{Y}$ of $\left(\mathrm{X}_{\mathcal{H}}\right)_{\mathrm{T}}$ is an intersection of locally closed subsets (respectively subschemes) $\left\{\mathrm{Y}_{i}\right\}_{i \in I}$ of $\left(\mathrm{X}_{\mathcal{H}}\right)_{\mathrm{T}}$, where each $\mathrm{Y}_{i}$ is a well-positioned subset (respectively subscheme) of $\left(\mathrm{X}_{\mathcal{H}}\right)_{\mathrm{T}}$ associated with $\mathrm{Y}_{i}^{\natural}:=\left\{\mathrm{Y}_{i, \mathrm{Z}}^{\natural}\right\}_{\mathrm{Z}}$ as in Definition 2.2.1. Then $\mathrm{Y}$ is also a well-positioned subset (respectively subscheme), associated with $\mathrm{Y}^{\natural}:=\left\{\bigcap_{i \in I} Y_{i, Z}^{\natural}\right\}_{z}$ as in Definition 2.2.1.

LEMMA 2.2.4. Suppose $\mathrm{Y}$ is a well-positioned subset (respectively subscheme) of $\left(\mathrm{X}_{\mathcal{H}}\right)_{\mathrm{T}}$, associated with $\mathrm{Y}^{\natural}=\left\{\mathrm{Y}_{\mathrm{Z}}^{\natural}\right\}_{\mathrm{Z}}$ as in Definition 2.2.1. If $\mathrm{Y}_{0}$ is a closed 
subset of $\mathrm{Y}$ that is a well-positioned subset of $\left(\mathrm{X}_{\mathcal{H}}\right)_{\mathrm{T}}$ associated with $\mathrm{Y}_{0}^{\natural}=\left\{\mathrm{Y}_{0, \mathrm{Z}}^{\natural}\right\}_{\mathrm{Z}}$, where each $\mathrm{Y}_{0, Z}^{\natural}$ is a closed subset of $\mathrm{Y}_{Z}^{\natural}$, then the open complement $\mathrm{Y}-\mathrm{Y}_{0}$ is a well-positioned subset (respectively subscheme), associated with $\left\{Y_{Z}^{\natural}-Y_{0, Z}^{\natural}\right\}_{Z}$. Similarly, if $\mathrm{Y}^{0}$ is an open subset of $\mathrm{Y}$ that is a well-positioned subset of $\left(\mathrm{X}_{\mathcal{H}}\right)_{\mathrm{T}}$ associated with $\mathrm{Y}^{0, \natural}=\left\{\mathrm{Y}_{\mathrm{Z}}^{0, \natural}\right\}_{\mathrm{Z}}$, where each $\mathrm{Y}_{\mathrm{Z}}^{0, \natural}$ is an open subset of $\mathrm{Y}_{\mathrm{Z}}^{\natural}$, then the closed complement $\mathrm{Y}-\mathrm{Y}^{0}$ is a well-positioned subset of $\left(\mathrm{X}_{\mathcal{H}}\right)_{\mathrm{T}}$, associated with $\left\{Y_{Z}^{\natural}-Y_{Z}^{0, \natural}\right\}_{Z}$

LEMMA 2.2.5. Suppose a locally closed subset (respectively subscheme) $\mathrm{Y}$ of $\left(\mathrm{X}_{\mathcal{H}}\right)_{\mathrm{T}}$ is a union of its open subsets (respectively subschemes) $\left\{Y_{i}\right\}_{i \in I}$, where each $\mathrm{Y}_{i}$ is a well-positioned subset (respectively subscheme) of $\left(\mathrm{X}_{\mathcal{H}}\right)_{\mathrm{T}}$ associated with $Y_{i}^{\natural}:=\left\{Y_{i, Z}^{\natural}\right\}_{Z}$ as in Definition 2.2.1. In the case of subschemes, suppose moreover that, for each $\mathrm{Z}$, the subschemes $\left\{Y_{i, \mathrm{Z}}^{\natural}\right\}_{i \in I}$ induce compatible open subscheme structures over their finite intersections, so that $\bigcup_{i \in I} Y_{i, \mathrm{Z}}^{\natural}$ is defined. Then $\mathrm{Y}$ is also a well-positioned subset (respectively subscheme), associated with $Y^{\natural}=\left\{\bigcup_{i \in I} Y_{i, Z}^{\natural}\right\}_{Z}$ as in Definition 2.2.1.

LEMMA 2.2.6. Suppose $\mathrm{Y}$ is a closed well-positioned subset (respectively subscheme) of $\left(\mathrm{X}_{\mathcal{H}}\right)_{\mathrm{T}}$, and suppose $\mathrm{Y}^{\natural}=\left\{\mathrm{Y}_{\mathrm{Z}}^{\natural}\right\}_{\mathrm{Z}}$ is associated with $\mathrm{Y}$ as in Definition 2.2.1. Then $\mathrm{Y}_{\mathrm{Z}}^{\natural}$ is closed in $\mathrm{Z}_{\mathrm{T}}$, and $\mathrm{Y}_{C}^{\natural}$ is closed in $C_{\mathrm{T}}$, for each $\mathrm{Z}$.

Proof. For each $\mathrm{Z}$, since $C \rightarrow \mathrm{Z}$ is proper and surjective, it suffices to show that $\mathrm{Y}_{C}^{\natural}$ is closed in $C_{\mathrm{T}}$. Assume otherwise, aiming for a contradiction. Then there exists a point $x$ in the closure of $Y_{C}^{\natural}$ in $C_{\mathrm{T}}$, but not in $Y_{C}^{\natural}$. Since $\Xi$ is fiberwise dense in $\Xi(\sigma)$ over $C$, and since $\Xi \rightarrow C$ and $\Xi_{\sigma} \rightarrow C$ are faithfully flat, by [21, IV-2, 2.3.12], there exists a point $y$ in the fiber of $\left(\Xi_{\sigma}\right)_{\mathrm{T}} \rightarrow C_{\mathrm{T}}$ above $x$ such that, for every affine neighborhood $U$ of $y$ in $\Xi(\sigma)_{\mathrm{T}}$, the pullback of $Y_{C}^{\natural}$ to $U^{0}:=$ $U \cap \Xi_{\mathrm{T}}$ is not closed. Since $\mathfrak{X}_{\sigma}=(\Xi(\sigma))_{\Xi_{\sigma}} \rightarrow \Xi(\sigma)$ is flat and induces the identity morphism along $\boldsymbol{\Xi}_{\sigma}$, for some $W$ as in Proposition 2.1.3, which we may assume to contain $y$, the pullback $Y_{W^{0}}^{\natural}$ of $Y_{C}^{\natural}$ to $\left(W^{0}\right)_{\mathrm{T}}$ is not closed. But this contradicts the assumption that $Y$ is closed, because $Y_{W^{0}}^{\natural}$ is by definition also the pullback of $Y$ to $\left(W^{0}\right)_{\mathrm{T}}$, as desired.

LEMMA 2.2.7. Suppose $\mathrm{Y}$ is a well-positioned subset (respectively subscheme) of $\left(\mathrm{X}_{\mathcal{H}}\right)_{\mathrm{T}}$, and suppose $\mathrm{Y}^{\natural}=\left\{\mathrm{Y}_{\mathrm{Z}}^{\natural}\right\}_{\mathrm{Z}}$ is associated with $\mathrm{Y}$ as in Definition 2.2.1. Then the closure (respectively schematic closure; see [10, Section 2.5, page 55]) $\overline{\mathrm{Y}}$ of $\mathrm{Y}$ in $\left(\mathrm{X}_{\mathcal{H}}\right)_{\mathrm{T}}$ is a well-positioned subset (respectively subscheme) if and only if the following condition holds: For each $\mathrm{Z}$, the closure (respectively schematic closure) $\bar{Y}_{C}^{\natural}$ of $Y_{C}^{\natural}$ in $C$ is the pullback of the closure (respectively schematic 
closure) $\bar{Y}_{Z}^{\natural}$ of $Y_{Z}^{\natural}$ in $\mathrm{Z}$, as a subset (respectively subscheme). In this case, the closed complement $\mathrm{Y}_{0}:=\overline{\mathrm{Y}}-\mathrm{Y}$ is a well-positioned subset. (The above condition is automatically satisfied when $\mathrm{Y}$ is closed in $\left(\mathrm{X}_{\mathcal{H}}\right)_{\mathrm{T}}$, by Lemma 2.2.6; or when $C \rightarrow \mathrm{Z}$ is flat for each Z, by [21, IV-2, 2.3.10] and [10, Section 2.5, Proposition 2]. Note that the schematic closures are defined because $\mathrm{T}$ is locally noetherian, in which case the locally closed immersions $\mathrm{Y} \rightarrow\left(\mathrm{X}_{\mathcal{H}}\right)_{\mathrm{T}}, \mathrm{Y}_{C}^{\natural} \rightarrow C_{\mathrm{T}}$, and $\mathrm{Y}_{\mathrm{Z}}^{\natural} \rightarrow \mathrm{Z}_{\mathrm{T}}$ are automatically quasicompact, for all $\mathrm{Z}$.) In this case, $\bar{Y}^{\natural}:=\left\{\bar{Y}_{Z}^{\natural}\right\}_{Z}$ (respectively $Y_{0}^{\natural}:=\left\{\bar{Y}_{Z}^{\natural}-Y_{Z}^{\natural}\right\}_{Z}$ ) is associated with $\bar{Y}$ (respectively $Y_{0}$ ) as in Definition 2.2.1.

Proof. For each $W$ as in Proposition 2.1.3, let $Y_{W^{0}}$ denote the pullback of $Y$ to $\left(W^{0}\right)_{\mathrm{T}}$. Since $W^{0} \rightarrow \mathrm{X}_{\mathcal{H}}$ is flat, by [21, IV-2, 2.3.10] (respectively [10, Section 2.5, Proposition 2]), the closure (respectively schematic closure) $\bar{Y}_{W^{0}}$ of $Y_{W^{0}}$ in $\left(W^{0}\right)_{\mathrm{T}}$ coincides with the pullback of $\bar{Y}$. Similarly, since $W^{0} \rightarrow \Xi$ and $\Xi \rightarrow C$ are flat, $\bar{Y}_{W^{0}}$ coincides with the pullback of $\bar{Y}_{C}^{\natural}$. Hence, by definition, $\bar{Y}$ is well positioned if and only if the condition in the lemma holds.

DEFINITION 2.2.8. Let $\left\{S_{i}\right\}_{i \in I}$ be a finite set of subschemes of a scheme $S$ such that each $S_{i}$ is a closed subset of the set-theoretic union $\bigcup_{i \in I} S_{i}$. For each $i \in I$, let $\bar{S}_{i}$ denote the schematic closure of $S_{i}$ in $S$, and let $S_{i, 0}:=\bar{S}_{i}-S_{i}$ denote the closed complement with its reduced subscheme structure. Then we define the union $\bigcup_{i \in I} S_{i}$ as a scheme as the (closed) schematic image of $\bigsqcup_{i \in I} \bar{S}_{i} \rightarrow S$ (see [10, Section 2.5, page 55]) subtracted by the closed subset $\bigcap_{i \in I} S_{i, 0}$.

LEMMA 2.2.9. Suppose a locally closed subset (respectively subscheme) $\mathrm{Y}$ of $\left(\mathrm{X}_{\mathcal{H}}\right)_{\mathrm{T}}$ is a finite union of its closed subsets (respectively subschemes) $\left\{\mathrm{Y}_{i}\right\}_{i \in I}$ (see Definition 2.2.8), where each $Y_{i}$ is a well-positioned subset (respectively subscheme) of $\left(\mathrm{X}_{\mathcal{H}}\right)_{\mathrm{T}}$. For each $i \in I$, suppose $\mathrm{Y}_{i}^{\natural}=\left\{\mathrm{Y}_{i, \mathrm{Z}}^{\natural}\right\}_{\mathrm{Z}}$ is associated with $\mathrm{Y}_{i}$ as in Definition 2.2.1. In the case of subschemes, suppose moreover that $C \rightarrow Z$ is flat, for each $\mathrm{Z}$. Then $\mathrm{Y}$ is also a well-positioned subset (respectively subscheme), and $Y^{\natural}:=\left\{Y_{Z}^{\natural}\right\}_{Z}$, where $Y_{Z}^{\natural}:=\bigcup_{i \in I} Y_{i, Z}^{\natural}$ as a subset (respectively subscheme) for each $\mathrm{Z}$, is associated with $\mathrm{Y}$ as in Definition 2.2.1. (Implicit in this statement is that each $Y_{Z}^{\natural}$ is defined in the case of subschemes.)

Proof. Suppose $x_{0}$ is any point of $Y_{i_{0}, Z}$ which specializes to a point $x_{1}$ of $Y_{i_{1}, Z}$, for some $i_{0}, i_{1} \in I$. For each $\sigma \in \Sigma_{\mathrm{Z}}^{+}$, since $C \rightarrow \mathrm{Z}$ is proper and surjective, since $\Xi$ is fiberwise dense in $\Xi(\sigma)$ over $C$, and since $\Xi \rightarrow C$ and $\Xi_{\sigma} \rightarrow C$ are faithfully flat, there exist some $W$ as in Proposition 2.1.3 and some points $\tilde{x}_{0}$ and $\tilde{x}_{1}$ of $W^{0}$ lifting $x_{0}$ and $x_{1}$, respectively, such that $\tilde{x}_{0}$ specializes to $\tilde{x}_{1}$. But $\tilde{x}_{0}$ and $\tilde{x}_{1}$ belong to the pullbacks of $Y_{i_{0}}$ and $Y_{i_{1}}$, respectively. Therefore, the 
assumption that $Y_{i_{0}}$ is closed in $Y$ shows that $\widetilde{x}_{1}$ is contained in the pullback of $Y_{i_{0}}$, and so its image $x_{1}$ is contained in $Y_{i_{0}, z}$. Hence, $Y_{i_{0}, Z}^{\natural}$ is closed in the set-theoretic union $Y_{Z}^{\natural}:=\bigcup_{i \in I} Y_{i, Z}^{\natural}$, and this union is locally closed as a subset of $Z_{\mathrm{T}}$. In the case of subsets, $Y^{\natural}:=\left\{Y_{Z}^{\natural}\right\}_{Z}$ is associated with $Y$ as in Definition 2.2.1. In the case of subschemes, each $Y_{Z}^{\natural}$ also admits the structure as a subscheme of $Z_{T}$ by Definition 2.2.8, and $Y^{\natural}:=\left\{Y_{Z}^{\natural}\right\}_{Z}$ is associated with $Y$ as in Definition 2.2.1 by [10, Section 2.5, Proposition 2], because $W^{0} \rightarrow \mathrm{X}_{\mathcal{H}}$ and $W^{0} \rightarrow C$ are flat, and because $C \rightarrow \mathrm{Z}$ is flat by assumption.

LEMMA 2.2.10. If $\mathrm{Y}$ is a well-positioned subset (respectively subscheme) of $\left(\mathrm{X}_{\mathcal{H}}\right)_{\mathrm{T}}$, if $\mathrm{Y}^{\natural}=\left\{\mathrm{Y}_{Z}^{\natural}\right\}_{\mathrm{Z}}$ is associated with $\mathrm{Y}$ as in Definition 2.2.1, and if $C \rightarrow \mathrm{Z}$ is reduced (that is, is flat and has geometrically reduced fibers; see [21, IV-2, 6.8.1]) for all $\mathrm{Z}$, then the unique reduced subscheme $\mathrm{Y}_{\text {red }}$ over the underlying locally closed subset $\mathrm{Y}$ of $\left(\mathrm{X}_{\mathcal{H}}\right)_{\mathrm{T}}$ is a well-positioned subscheme, and $\mathrm{Y}_{\text {red }}^{\natural}=\left\{\mathrm{Y}_{\text {red, } \mathrm{Z}}^{\natural}\right\}_{\mathrm{Z}}$, where $Y_{\text {red, } Z}^{\natural}:=\left(Y_{Z}^{\natural}\right)_{\text {red }}$ for each $Z$, is associated with $Y_{\text {red }}$ as in Definition 2.2.1. Moreover, if $\mathrm{T}=\operatorname{Spec}(k)$ for some field $k$, and if $C_{\mathrm{T}} \rightarrow Z_{\mathrm{T}}$ is (proper and) smooth for all $\mathrm{Z}$, then the smooth locus $\mathrm{Y}_{\mathrm{sm}}$ of $\mathrm{Y}_{\text {red }}$ is a well-positioned subscheme, and $\mathrm{Y}_{\mathrm{sm}}^{\natural}=\left\{\mathrm{Y}_{\mathrm{sm}, \mathrm{Z}}^{\natural}\right\}_{\mathrm{Z}}$, where $\mathrm{Y}_{\mathrm{sm}, \mathrm{Z}}^{\natural}:=\left(\mathrm{Y}_{\mathrm{Z}}^{\natural}\right)_{\mathrm{sm}}$ is the smooth locus of $\left(\mathrm{Y}_{\mathrm{Z}}^{\natural}\right)_{\text {red }}$ for each $\mathrm{Z}$, is associated with $\mathrm{Y}_{\mathrm{sm}}$ as in Definition 2.2.1.

Proof. For each $W$ as in Proposition 2.1.3, by the regularity of $W \rightarrow\left(\mathrm{X}_{\mathcal{H}}\right)_{\mathrm{T}}$ and $W \rightarrow \Xi(\sigma)$ (see [21, IV-2, 7.8.3(v)]), by the reducedness of $C \rightarrow \mathrm{Z}$, and by $\left[21, \mathrm{IV}-2,5.8 .5,6.4 .1\right.$, and 6.5.3], the pullback of $Y_{\text {red }}$ to $\left(W^{0}\right)_{\mathrm{T}}$ coincides with the pullback of $\left(Y_{Z}^{\natural}\right)_{\text {red }}$ as reduced subschemes, because their underlying sets already coincide. Hence, $Y_{\text {red }}$ is a well-positioned subscheme. If $\mathrm{T}=\operatorname{Spec}(k)$ and if $C_{\mathrm{T}} \rightarrow \mathrm{Z}_{\mathrm{T}}$ is smooth (which is, in particular, also regular), by [21, IV-2, 6.5.3] again, the pullback of $Y_{s m}$ to $\left(W^{0}\right)_{T}$ coincides with the pullback of the smooth locus $\left(Y_{Z}^{\natural}\right)_{\text {sm }}$ of $\left(Y_{Z}^{\natural}\right)_{\text {red }}$. Since $Y_{\text {sm }}$ is open in $Y_{\text {red }}$ (by [21, IV-2, 6.12.5]), it is locally closed, and hence also a well-positioned subscheme.

REMARK 2.2.11. It is natural to ask whether the collection $Y^{\natural}$ in Definition 2.2.1 is uniquely determined by $Y$. In the case of subsets, this is true by definition. In the case of subschemes, it is still true if $C \rightarrow Z$ is flat-see (4) of Theorem 2.3.2 below.

2.3. Partial compactifications of well-positioned subschemes. Let $T$ be a locally noetherian scheme over $\mathrm{S}$, as in the beginning of Section 2.2.

DEFINITION 2.3.1. Suppose $Y$ is a locally closed subset (respectively subscheme) of $\left(\mathrm{X}_{\mathcal{H}}\right)_{\mathrm{T}}$. Let $\overline{\mathrm{Y}}$ denote the closure (respectively schematic closure) of $\mathrm{Y}$ in $\left(\mathrm{X}_{\mathcal{H}}\right)_{\mathrm{T}}$, 
and let $Y_{0}$ denote the complement subset $\bar{Y}-Y$. (In this definition, we do not assume that any of $Y, \bar{Y}$, or $Y_{0}$ is well positioned.) Let $\bar{Y}^{\min }$ denote the closure (respectively schematic closure) of $Y$ (or equivalently $\bar{Y}$ ) in $\left(X_{\mathcal{H}}^{\min }\right)_{\mathrm{T}}$, let $\bar{Y}_{\Sigma}^{\text {tor }}$ denote the closure (respectively schematic closure) of $Y$ (or equivalently $\bar{Y}$ ) in $\left(X_{\mathcal{H}, \Sigma}^{\text {tor }}\right)_{\mathrm{T}}$, let $Y_{0}^{\min }$ denote the closure of $Y_{0}$ in $\left(X_{\mathcal{H}}^{\min }\right)_{\mathrm{T}}$, and let $Y_{0, \Sigma}^{\text {tor }}$ denote the closure of $\mathrm{Y}_{0}$ in $\left(\mathrm{X}_{\mathcal{H}, \Sigma}^{\text {tor }}\right)_{\mathrm{T}}$. In the case of subsets, we view them as subschemes with their reduced subscheme structures. Let $Y^{\min }:=\bar{Y}^{\min }-Y_{0}^{\min }$ and $Y_{\Sigma}^{\text {tor }}:=\bar{Y}_{\Sigma}^{\text {tor }}-Y_{0, \Sigma}^{\text {tor }}$, with induced open immersions $J_{Y^{\min }}: \mathrm{Y} \hookrightarrow \mathrm{Y}^{\min }$ and $J_{Y_{\Sigma}^{\text {tor }}}: \mathrm{Y} \hookrightarrow \mathrm{Y}_{\Sigma}^{\text {tor }}$ over $\mathrm{T}$. We shall call $\mathrm{Y}^{\mathrm{min}}$ the partial minimal compactification of $\mathrm{Y}$, and $\mathrm{Y}_{\Sigma}^{\text {tor }}$ the partial toroidal compactification of $\mathrm{Y}$, with the term partial suppressed when $\mathrm{Y}$ is a closed subscheme of $\left(X_{\mathcal{H}}\right)_{\mathrm{T}}$. (These partial compactifications are canonically determined by $\mathrm{X}_{\mathcal{H}} \stackrel{J_{\mathrm{X}_{\mathcal{H}}^{\mathrm{w}}, \Sigma}}{\hookrightarrow} \mathrm{X}_{\mathcal{H}, \Sigma}^{\text {tor }} \stackrel{\oint_{\mathcal{H}, \Sigma}}{\rightarrow} \mathrm{X}_{\mathcal{H}}^{\text {min }}, \mathrm{T}$, and $\mathrm{Y}$, by their very constructions.)

Theorem 2.3.2 (Cf. [45, Proposition 2.2] or Proposition 2.1.2). For each wellpositioned subset (respectively subscheme) $\mathrm{Y}$ of $\left(\mathrm{X}_{\mathcal{H}}\right)_{\mathrm{T}}$ with a collection $\mathrm{Y}^{\natural}=$ $\left\{Y_{Z}^{\natural}\right\}_{Z}$ as in Definition 2.2.1, its partial minimal and toroidal compactifications

$$
J_{Y^{\min }}: \mathrm{Y} \hookrightarrow \mathrm{Y}^{\min }
$$

and

$$
J_{Y_{\Sigma}^{\text {tor }}}: \mathrm{Y} \hookrightarrow \mathrm{Y}_{\Sigma}^{\text {tor }}
$$

as in Definition 2.3.1 satisfy the following properties:

(1) For each $\Sigma$, the proper surjective structural morphism $\oint_{\mathcal{H}, \Sigma}: \mathrm{X}_{\mathcal{H}, \Sigma}^{\text {tor }} \rightarrow \mathrm{X}_{\mathcal{H}}^{\min }$ induces a proper surjective structural morphism

$$
\oint_{Y, \Sigma}: Y_{\Sigma}^{\text {tor }} \rightarrow Y^{\min }
$$

(over $\mathrm{T})$, so that

$$
J_{Y^{\min }}=\oint_{Y, \Sigma} \circ J_{Y_{\Sigma}^{\text {tor }}} .
$$

The structural morphisms $\mathrm{Y}^{\mathrm{min}} \rightarrow \mathrm{T}$ and $\mathrm{Y}_{\Sigma}^{\text {tor }} \rightarrow \mathrm{T}$ are projective when $\mathrm{Y}$ is closed in $\left(\mathrm{X}_{\mathcal{H}}\right)_{\mathrm{T}}$ (under the assumption in (4) of Proposition 2.1.2 that $\Sigma$ is projective).

(2) Consider an ample invertible sheaf $\omega_{\mathrm{X}_{\mathcal{H}}^{\min }}$ over $\mathrm{X}_{\mathcal{H}}^{\min }$ chosen as follows: In Case $(\mathrm{Sm})$, we take $\omega_{\mathrm{X}_{\mathcal{H}}^{\min }}$ to be the pullback of $\omega$ as in [36, Theorem 7.2.4.1(2)]. In Case $(\mathrm{Nm})$, we take $\omega_{\mathrm{X}_{\mathcal{H}}^{\min }}$ to be the pullback of $\omega_{\overrightarrow{\mathrm{M}}_{\mathcal{H}}, \mathrm{J}}$ as 
in [38, Proposition 6.4]. In Case $(\mathrm{Spl})$, we take $\omega_{\mathrm{X}_{\mathcal{H}}^{\min }}$ to be the pullback of any of the ample $\omega_{\overrightarrow{\mathrm{M}}_{\mathcal{H}}^{\text {spl,min }}, \mathrm{J}}^{\otimes(k, \mu)}$ as in [43, Theorem 4.3.1(3)]. In Case (Hdg), we take $\omega_{\mathrm{X}_{\mathcal{H}}^{\min }}$ to be the pullback of $\omega_{K}^{\min }$ as in [50, Theorem 5.2.11(2)]. Then the pullback $\omega_{\mathrm{Y}_{\min }}$ of $\omega_{\mathrm{X}_{\mathcal{H}}^{\min }}$ to $\mathrm{Y}^{\min }$ is relatively ample over $\mathrm{T}$, and its further pullback $\omega_{Y_{\Sigma}^{\text {tor }}}$ to $\mathrm{Y}_{\Sigma}^{\text {tor }}$ is semiample over the preimage of every affine open subscheme of $\mathrm{T}$. When $\mathrm{T}$ is affine and $\mathrm{Y}$ is closed in $\left(\mathrm{X}_{\mathcal{H}}\right)_{\mathrm{T}}$, we have canonical morphisms

$$
\begin{aligned}
Y_{\Sigma}^{\text {tor }} & \rightarrow \operatorname{Proj}\left(\bigoplus_{k \geqslant 0} \Gamma\left(\mathrm{Y}_{\Sigma}^{\text {tor }}, \omega_{\mathrm{Y}_{\Sigma}^{\text {tot }}}^{\otimes k}\right)\right) \\
& \rightarrow \operatorname{Proj}\left(\bigoplus_{k \geqslant 0} \Gamma\left(\mathrm{Y}^{\text {min }}, \omega_{\mathrm{Y}^{\text {min }}}^{\otimes k}\right)\right) \cong \mathrm{Y}^{\text {min }},
\end{aligned}
$$

which coincides with the Stein factorization of $\oint_{\mathrm{Y}, \Sigma}: \mathrm{Y}_{\Sigma}^{\text {tor }} \rightarrow \mathrm{Y}^{\mathrm{min}}$.

(3) The stratification of $\mathrm{X}_{\mathcal{H}}^{\min }$ by locally closed subschemes $\mathrm{Z}$ induces a stratification of $\mathrm{Y}^{\mathrm{min}}$ by locally closed subschemes

$$
\mathrm{Y}_{\mathrm{Z}}:=\mathrm{Z} \times_{\mathrm{X}_{\mathcal{H}}^{\min }} \mathrm{Y}^{\min }
$$

each of which is equipped with a canonical morphism $Y_{Z}^{\natural} \rightarrow Y_{Z}$ which induces a bijection between the underlying subsets of $\mathrm{Z}_{\mathrm{T}}$ (see Definition 2.2.1), with an open dense stratum $\mathrm{Y}_{Z}^{\natural}=\mathrm{Y}$ for $\mathrm{Z}=\mathrm{X}_{\mathcal{H}}$. For each $\Sigma$, the stratification of $\mathrm{X}_{\mathcal{H}, \Sigma}^{\text {tor }}$ by locally closed subschemes $Z_{[\sigma]}$ induces a stratification of $\mathrm{Y}_{\Sigma}^{\text {tor }}$ by locally closed subschemes

$$
\mathrm{Y}_{\mathrm{Z}_{[\sigma]}}:=\mathrm{Z}_{[\sigma]} \times_{\mathrm{X}_{\mathcal{H}, \Sigma}^{\text {tor }}} \mathrm{Y}_{\Sigma}^{\text {tor }}
$$

with an open dense stratum $\mathrm{Y}_{\mathrm{Z}_{[00]]}}=\mathrm{Y}$ for $\mathrm{Z}=\mathrm{X}_{\mathcal{H}}$ and $\sigma=\{0\}$. For each $Z_{[\sigma]}$, the canonical surjective morphism $Z_{[\sigma]} \rightarrow Z$ induces a surjective morphism $\mathrm{Y}_{\mathrm{Z}_{[\sigma]}} \rightarrow \mathrm{Y}_{\mathrm{Z}}$ which factors through a (surjective) morphism $\mathrm{Y}_{\mathrm{Z}_{[\sigma]}} \rightarrow \mathrm{Y}_{\mathrm{Z}}^{\natural}$ (which is the pullback of $\mathrm{Z}_{[\sigma]} \rightarrow \mathrm{Z}$ when $\mathrm{Y}$ is a well-positioned subscheme of $\left.\left(\mathrm{X}_{\mathcal{H}}\right)_{\mathrm{T}}\right)$. Hence, $\mathrm{Y}_{\mathrm{Z}_{[\sigma]}}$ is nonempty exactly when $\mathrm{Y}_{Z}^{\natural}$ is, and exactly when $\mathrm{Y}_{\mathrm{Z}}$ is.

(4) For each top-dimensional cone $\sigma$ in $\Sigma_{Z}^{+}$, we have a canonical isomorphism $\mathrm{Y}_{\mathrm{Z}_{[\sigma]}} \stackrel{\sim}{\rightarrow} \mathrm{Y}_{C}^{\natural}$, which shows that $\mathrm{Y}_{C}^{\natural}$ is determined by $\mathrm{Y}$, for each $\mathrm{Z}$. Thus, in the case of subschemes, if the surjection $C \rightarrow \mathrm{Z}$ is flat, then $\mathrm{Y}_{\mathrm{Z}}^{\natural}$ is determined by its pullback $Y_{C}^{\natural}$ under a faithfully flat morphism, and hence also by $\mathrm{Y}$. In the case of subsets, $\mathrm{Y}_{Z}^{\natural}$ is determined by $\mathrm{Y}_{C}^{\natural}$ and $\mathrm{Y}$ by definition, without the flatness assumption on $C \rightarrow Z$. (See Remark 2.2.11.) 
(5) For each representative $\sigma \in \Sigma_{Z}^{+}$of an orbit $[\sigma] \in \Sigma_{Z}^{+} / \Gamma$, and for ? = $\Xi, \Xi(\sigma), \Xi_{\sigma}, \mathfrak{X}_{\sigma}, \mathfrak{X}_{\sigma}^{\circ}$, and $\mathfrak{X}_{\Sigma_{\mathrm{Z}}}$, let $Y_{?}^{\natural}$ denote the pullback of ? under the canonical morphism $Y_{C}^{\natural} \rightarrow C$. (In the case of subsets, we view $Y_{Z}^{\natural}$ and $\mathrm{Y}_{C}^{\natural}$ as subschemes of $\mathrm{Z}$ and $C$, respectively, with their reduced subscheme structures, so that the above all make sense as statements for schemes and formal schemes. In this case, $C \rightarrow \mathrm{Z}$ induces a proper surjective morphism $\mathrm{Y}_{C}^{\natural} \rightarrow \mathrm{Y}_{\mathrm{Z}}^{\natural}$ of schemes, which is the pullback of $C \rightarrow \mathrm{Z}$ when $C \rightarrow \mathrm{Z}$ is reduced; that is, is flat and has geometrically reduced fibers, as in [21, IV-2, 6.8.1].) Then we have a canonical isomorphism

$$
\mathrm{Y}_{\mathfrak{X}_{\sigma}}^{\natural} \stackrel{\sim}{\rightarrow}\left(\mathrm{Y}_{\Sigma}^{\text {tor }}\right)_{\mathrm{Y}_{\mathrm{Z}_{[\sigma]}}}^{\wedge}
$$

induced by the canonical isomorphism $\mathfrak{X}_{\sigma} \stackrel{\sim}{\rightarrow}\left(\mathrm{X}_{\mathcal{H}, \Sigma}^{\text {tor }}\right) \hat{\mathrm{Z}}_{[\sigma]}$, extending a canonical isomorphism $\mathrm{Y}_{\Xi_{\sigma}}^{\natural} \stackrel{\sim}{\rightarrow} \mathrm{Y}_{\mathrm{Z}_{[\sigma]}}$ induced by the canonical isomorphism $\Xi_{\sigma} \stackrel{\sim}{\rightarrow} Z_{[\sigma]}$ (see (8) of Proposition 2.1.2), which extends to flat morphisms

$$
\mathrm{Y}_{\mathfrak{X}_{\sigma}}^{\natural} \rightarrow \mathrm{Y}_{\mathfrak{X}_{\sigma}^{\circ}}^{\natural} \hookrightarrow \mathrm{Y}_{\mathfrak{X}_{\Sigma_{\mathrm{Z}}}^{\natural}}^{\natural} \rightarrow \mathrm{Y}_{\Sigma}^{\text {tor }}
$$

induced by (2.1.4), inducing compatible canonical isomorphisms

$$
\mathrm{Y}_{\mathfrak{X}_{\sigma}^{\natural}}^{\natural} \stackrel{\sim}{\rightarrow}\left(\mathrm{Y}_{\Sigma}^{\text {tor }}\right)_{\bigcup_{\tau \in \Sigma_{\mathrm{Z}}^{+}, \bar{\tau} \subset \bar{\sigma}}^{\wedge}} \mathrm{Y}_{\left[\mathrm{Z}_{[\tau]}\right.}
$$

and

$$
\mathrm{Y}_{\mathfrak{X}_{\Sigma \mathrm{Z}}}^{\natural} / \Gamma \stackrel{\sim}{\rightarrow}\left(\mathrm{Y}_{\Sigma}^{\text {tor }}\right)_{\bigcup_{[\tau] \in \Sigma_{\mathrm{Z}}^{+} / \Gamma}^{+}}^{\wedge} \mathrm{Y}_{[\tau]}
$$

induced by (2.1.5) and (2.1.6), respectively.

(6) For each $\sigma \in \Sigma_{Z}^{+}$, and for each affine open formal subscheme $\operatorname{Spf}(R)$ of $\mathrm{Y}_{\mathfrak{X}_{\sigma}}^{\natural}$, under the canonically induced (flat) morphisms $\operatorname{Spec}(R) \rightarrow \mathrm{Y}_{\Sigma}^{\text {tor }}$ and $\operatorname{Spec}(R) \rightarrow Y_{E(\sigma)}^{\natural}$ induced by (2.3.4), the stratification of $\operatorname{Spec}(R)$ induced by that of $\mathrm{Y}_{\Sigma}^{\text {tor }}$ coincides with the stratification of $\operatorname{Spec}(R)$ induced by that of $Y_{\Xi(\sigma)}^{\natural}$. In particular, the preimages of $Y_{\text {and }} Y_{\Xi}^{\natural}$ coincide as open subschemes of $\operatorname{Spec}(R)$. Analogous statements are true for $Y_{\mathfrak{X}_{\sigma}^{\circ}}^{\natural}$ and (2.3.6).

(7) Let $x$ be a point of $Y_{\Xi_{\sigma}}^{\natural}$, which can be canonically identified with a point of $\mathrm{Y}_{\mathrm{Z}_{[\sigma]}}$ via the above isomorphism. Then there exists an étale neighborhood

$$
\bar{U} \rightarrow \mathrm{Y}_{\Sigma}^{\text {tor }}
$$

of $x$ and an étale morphism

$$
\bar{U} \rightarrow Y_{\Xi(\sigma)}^{\natural}
$$


respecting $x$ such that the stratification of $\bar{U}$ induced by that of $\mathrm{Y}_{\Sigma}^{\text {tor }}$ coincides with the stratification of $\bar{U}$ induced by that of $Y_{\Xi(\sigma)}^{\natural}$, or rather by that of $\Xi(\sigma)$ as in (9) of Proposition 2.1.2; and such that the pullbacks of these étale morphisms to $\mathrm{Y}_{\mathrm{Z}_{[\sigma]}}$ and to $\mathrm{Y}_{\Xi_{\sigma}}^{\natural}$ are both open immersions. (In particular, $\mathrm{Y}_{\Sigma}^{\text {tor }}$ and $\mathrm{Y}_{\Xi(\sigma)}^{\natural}$, equipped with their stratifications as explained above, are étale locally isomorphic at $x$.) There also exist étale morphisms as above with the analogous but stronger property that their respective pullbacks to $\bigcup_{\tau \in \Sigma_{\mathrm{Z}}^{+}, \bar{\tau} \subset \bar{\sigma}} \mathrm{Y}_{\mathrm{Z}_{[\tau]}}$ and $\bigcup_{\tau \in \Sigma_{\mathrm{Z}}^{+}, \bar{\tau} \subset \bar{\sigma}} \mathrm{Y}_{\Xi_{\tau}}^{\natural}$ (with their reduced structures) are both open immersions.

Proof. The properties (1) and (2), and the assertions concerning underlying subsets in the property (3), follow immediately from the definitions.

Let $\bar{Y}, \bar{Y}^{\text {min }}, \bar{Y}_{\Sigma}^{\text {tor }}, Y_{0}, Y_{0}^{\text {min }}$, and $Y_{0, \Sigma}^{\text {tor }}$ be as in Definition 2.3.1. For each $Z$, let $\bar{Y}_{C}^{\natural}$ denote the closure (respectively schematic closure) of $Y_{C}^{\natural}$ in $C_{\mathrm{T}}$, let $Y_{C, 0}^{\natural}:=$ $\bar{Y}_{C}^{\natural}-Y_{C}^{\natural}$ denote the complement, let $\bar{Y}_{Z}^{\natural}$ denote the closure (respectively schematic closure) of $Y_{Z}^{\natural}$ in $Z_{T}$, and let $Y_{Z, 0}^{\natural}:=\bar{Y}_{Z}^{\natural}-Y_{Z}^{\natural}$ denote the complement. For each $W$ as in Proposition 2.1.3, as in the proof of Lemma 2.2.7, by [21, IV-2, 2.3.10], by the definition of closures (respectively schematic closures), and by the flatness of $W \rightarrow\left(\mathrm{X}_{\mathcal{H}, \Sigma}^{\text {tor }}\right)_{\mathrm{T}}$ and $W \rightarrow \Xi(\sigma) \rightarrow C$, the pullback of $\mathrm{Y}_{\Sigma}^{\text {tor }}=\overline{\mathrm{Y}}_{\Sigma}^{\text {tor }}-\mathrm{Y}_{0, \Sigma}^{\text {tor }}$ to $W_{\mathrm{T}}$ is the pullback of $Y_{C}^{\natural}=\bar{Y}_{C}^{\natural}-Y_{C, 0}^{\natural}$, as a subset (respectively subscheme). When $\mathrm{Y}$ is just given as a subset of $\left(\mathrm{X}_{\mathcal{H}}\right)_{\mathrm{T}}$, by the regularity of $W \rightarrow\left(\mathrm{X}_{\mathcal{H}, \Sigma}^{\text {tor }}\right)_{\mathrm{T}}$ and $W \rightarrow \Xi(\sigma)$ (see [21, IV-2, 6.8.1 and 7.8.3(v)]), by the normality of $\Xi(\sigma) \rightarrow C$ (see [38, Proposition 8.14] and its proof), and by [21, IV-2, 5.8.5, 5.8.6, 6.4.1, and 6.5.3], it follows that the pullback of $Y_{\Sigma}^{\text {tor }}$ to $W_{\mathrm{T}}$ also coincides with the pullback of $Y_{C}^{\natural}$ as reduced subschemes.

By the definition of $W=\operatorname{Spec}(R)$ by an affine open subscheme $\mathfrak{W}=\operatorname{Spf}(R)$ of $\mathfrak{X}_{\sigma}^{\circ}$, by Proposition 2.1.3, the pullback of $Y_{Z_{[\sigma]}}:=Z_{[\sigma]} \times_{\chi_{\mathcal{H}, \Sigma}^{\text {tor }}} Y_{\Sigma}^{\text {tor }}$ under the canonical isomorphism $\Xi_{\sigma} \stackrel{\sim}{\rightarrow} Z_{[\sigma]}$ coincides with the pullback of $Y_{C}^{\natural}$, for all $\sigma \in \Sigma_{\mathrm{Z}}^{+}$, and hence all the assertions in the properties (5) and (6) follow. Since the canonical morphism $\Xi_{\sigma} \rightarrow C$ is an isomorphism when $\sigma$ is top dimensional, this shows that $Y_{C}^{\natural}$ is uniquely determined by $Y$, and the remaining assertions in the property (4) also follow. Since the canonical morphisms $\Xi_{\sigma} \rightarrow C \rightarrow Z$ are surjective, whose composition can be identified with the surjection $Z_{[\sigma]} \rightarrow Z$ induced by $\oint_{\mathcal{H}, \Sigma}: X_{\mathcal{H}, \Sigma}^{\text {tor }} \rightarrow X_{\mathcal{H}}^{\text {min }}$, the induced morphisms

$$
\mathrm{Y}_{\mathrm{Z}_{[\sigma]}} \cong \mathrm{Y}_{\Xi_{\sigma}}^{\natural} \rightarrow \mathrm{Y}_{C}^{\natural} \rightarrow \mathrm{Y}_{\mathrm{Z}}^{\natural} \rightarrow \mathrm{Y}_{\mathrm{Z}}
$$

are also surjective, where the last morphism induces a bijection between subsets of $Z_{\mathrm{T}}$, and hence all assertions in the property (3) follow. 
Finally, let us prove the property (7). We may and we shall assume that $S$ is excellent, because the models in Assumption 2.1.1, and their compactifications in Proposition 2.1.2, are all defined over excellent Dedekind domains. Since $\mathbf{X}_{\mathcal{H}}^{\text {tor }}$ and $\Xi(\sigma)$ are of finite presentation over $\mathrm{S}$, by the usual limit argument, we may and we shall assume that $\mathrm{T}$ is of finite type over S. By Artin's approximation (see [2, Theorem 1.12, and the proof of the corollaries in Section 2]), and by the isomorphism (2.3.4) established above, we have some étale neighborhood $\bar{U} \rightarrow Y_{\Sigma}^{\text {tor }}$ of $x$ and an étale morphism $\bar{U} \rightarrow Y_{\Xi(\sigma)}^{\natural}$ such that the preimages of $Y_{Z_{[\sigma]}}$ and $Y_{\Xi_{\sigma}}^{\natural}$ coincide, such that the pullbacks of the étale morphisms to $Y_{Z_{[\sigma]}}$ and $Y_{\Xi_{\sigma}}^{\natural}$ are open immersions, and such that the stratifications induced by those of $X_{\mathcal{H}, \Sigma}^{\text {tor }}$ and $\Xi(\sigma)$ can be matched up to automorphisms of the completion of $\bar{U}$ along the common preimage of $Y_{Z_{[\sigma]}}$ and $Y_{\Xi_{\sigma}}^{\natural}$. The condition for such automorphisms to match stratifications (or rather some finite collections of closed subschemes) is equivalent to the solution of finitely many algebraic equations. Thus, by applying Artin's approximation again, up to modifying the choices of $\bar{U} \rightarrow \mathrm{Y}_{\Sigma}^{\text {tor }}$ and $\bar{U} \rightarrow Y_{\Xi(\sigma)}^{\natural}$, we may assume that the induced stratifications already coincide over $\bar{U}$, so that the first assertion of the property (7) holds. By using the isomorphism (2.3.6) instead of (2.3.4) in the above argument, we also obtain the other stronger assertion of the property (7), as desired.

REMARK 2.3.8. In Case (Sm), the assertions for $Y_{\Sigma}^{\text {tor }}$ in Theorem 2.3.2 show that our notion of well-positioned subschemes is consistent with the one introduced by Boxer in [12, Section 3.4].

REMARK 2.3.9. Shimura subvarieties are not well positioned in general, even as subsets, and that is why their compactifications (which can be constructed in a similar way) are more difficult to describe.

Thanks to the proof of Theorem 2.3.2, we can slightly weaken Definition 2.2.1 as follows:

LEMMA 2.3.10. Suppose T is a locally noetherian scheme over $\mathrm{S}$, and suppose $\mathrm{Y}$ is a locally closed subset of $\left(\mathrm{X}_{\mathcal{H}}\right)_{\mathrm{T}}$ such that, for each $\mathrm{Z}$, there exists some subset $\mathrm{Y}_{\mathrm{Z}}^{\natural}$ of $\mathrm{Z}_{\mathrm{T}}$ such that, for each $W$ as in Proposition 2.1.3, the pullback of $\mathrm{Y}_{\text {to }}\left(W^{0}\right)_{\mathrm{T}}$ coincides with the pullback of $\mathrm{Y}_{Z}^{\natural}$. Then $\mathrm{Y}_{Z}^{\natural}$ is automatically locally closed in $\mathrm{Z}_{\mathrm{T}}$.

Proof. Let $Y_{\Sigma}^{\text {tor }}$ be as in Definition 2.3.1. Since $C \rightarrow Z$ is proper and surjective, it suffices to show that the pullback $\mathrm{Y}_{C}^{\natural}$ of $\mathrm{Y}_{\mathrm{Z}}^{\natural}$ is locally closed in $C_{\mathrm{T}}$. Since $W \rightarrow$ $\mathrm{X}_{\mathcal{H}, \Sigma}^{\text {tor }}$ and $W \rightarrow \Xi(\sigma)$ are flat, by [21, IV-2, 2.3.10], the pullback of $Y_{\Sigma}^{\text {tor }}$ to $W_{s}$ 
also coincides with the pullback of $Y_{C}^{\natural}$. Since $W$ is arbitrary, we may and we shall assume that the $\sigma$ involved is top dimensional in $\Sigma_{Z}^{+}$, in which case we have an isomorphism $\mathrm{Y}_{\mathrm{Z}_{[\sigma]}} \stackrel{\sim}{\rightarrow} \mathrm{Y}_{C}^{\natural}$ induced by $\mathrm{Z}_{[\sigma]} \stackrel{\sim}{\rightarrow} C$, as in (4) of Theorem 2.3.2. Since $Y_{Z_{[\sigma]}}$ is locally closed in $\left(Z_{[\sigma]}\right)_{T}$ by its definition as a pullback of $Y_{\Sigma}^{\text {tor }}$, it follows that $Y_{C}^{\natural}$ is locally closed in $C_{\mathrm{T}}$, as desired.

Thanks to (7) of Theorem 2.3.2, we also have the following:

PROPOSITION 2.3.11. If $\mathrm{Y}$ is a well-positioned subset (respectively subscheme) of $\left(\mathrm{X}_{\mathcal{H}}\right)_{\mathrm{T}}$, and if $\mathrm{Y}_{C}^{\natural} \rightarrow \mathrm{Y}_{\mathrm{Z}}^{\natural}$ has connected fibers for all $\mathrm{Z}$, then the open-and-closed subsets (respectively subschemes) of $\mathrm{Y}$ are well-positioned subsets (respectively subschemes), and their closures in $\mathrm{Y}^{\min }$ and $\mathrm{Y}_{\Sigma}^{\text {tor }}$ are also open and closed in these partial compactifications.

Proof. Suppose $Y^{\natural}=\left\{Y_{Z}^{\natural}\right\}_{Z}$ is associated with $Y$ as in Definition 2.2.1. Suppose $Y_{1}$ is an open-and-closed subset (respectively subscheme) of $Y$. Let $Y_{2}:=Y-Y_{1}$ (with its open subscheme structure when $Y$ is a subscheme). We claim that their respective closures $Y_{1, \Sigma}^{\text {tor }}$ and $Y_{2, \Sigma}^{\text {tor }}$ in $Y_{\Sigma}^{\text {tor }}$ do not overlap.

Suppose, to the contrary, that there exist some $Z$ and $\sigma \in \Sigma_{Z}^{+}$with some point $x \in \mathrm{Y}_{\mathrm{Z}_{[\sigma]}} \cap \mathrm{Y}_{1, \Sigma}^{\text {tor }} \cap \mathrm{Y}_{2, \Sigma}^{\text {tor }}$, which we identify with a point of $\mathrm{Y}_{\Xi_{\sigma}}$ via the isomorphism $Y_{\Xi_{\sigma}}^{\natural} \cong Y_{Z_{[\sigma]}}$ in (5) of Theorem 2.3.2. Let $\bar{U} \rightarrow \mathrm{Y}_{\Sigma}^{\text {tor }}$ and $\bar{U} \rightarrow \mathrm{Y}_{\Xi(\sigma)}^{\natural}$ be étale morphisms as in (7) of Theorem 2.3.2, whose pullbacks to $Y_{Z_{[\sigma]}}$ and to $Y_{E_{\sigma}}^{\natural}$ are both open immersions. Up to replacing $\bar{U}$ with an open subscheme, we may and we shall assume that $\bar{U} \rightarrow \mathrm{Y}_{\Sigma}^{\text {tor }}$ and $\bar{U} \rightarrow \mathrm{Y}_{\Xi(\sigma)}^{\natural}$ have connected fibers. Consider the open subscheme $U:=\bar{U} \times_{\mathrm{Y}_{\Sigma}^{\text {tor }}} \mathrm{Y}$ of $\bar{U}$, which can be identified with $\bar{U} \times_{Y_{E(\sigma)}^{\natural}} Y_{\Xi}^{\natural}$ because the étale morphisms match stratification's. Let $y$ denote the image of $x$ in $Y_{C}^{\natural}$. Since $\bar{U} \rightarrow Y_{\Xi(\sigma)}^{\natural}$ is étale, and since $Y_{\Xi}^{\natural} \hookrightarrow Y_{\Xi(\sigma)}^{\natural}$ is an affine toroidal embedding over $Y_{C}^{\natural}$, which is fiberwise dense, for each $i$, there exists some point $x_{i}$ of the pullback of $Y_{i}$ to $U$ which specializes to $x$ in $\bar{U}$ and is mapped to $y$ in $Y_{C}^{\natural}$. Since $\Xi \rightarrow C$ is a torus torsor, the fiber of $U \rightarrow Y_{E}^{\natural} \rightarrow Y_{C}^{\natural}$ above $y$ is connected, which cannot overlap with both the pullbacks of $Y_{1}$ and $Y_{2}$. Hence, such an $x$ cannot exist, and the claim follows. So $Y_{1, \Sigma}^{\text {tor }}$ is also open and closed in $\mathrm{Y}_{\Sigma}^{\text {tor }}$.

Now consider $Y_{i, Z_{[\sigma]}}:=Y_{Z_{[\sigma]}} \cap Y_{i, \Sigma}^{\text {tor }}$, for each $i$. Since $Y_{C}^{\natural} \rightarrow Y_{Z}^{\natural}$ has connected fibers, so does the surjective morphism $Y_{Z_{[\sigma]}} \cong Y_{\Xi_{\sigma}}^{\natural} \rightarrow Y_{Z}^{\natural}$. Consequently, the image $Y_{i, Z}^{\natural}$ of $Y_{i, Z_{[\sigma]}}$ in $Y_{Z}^{\natural}$, which necessarily coincides with $Y_{Z} \cap Y_{i}^{\min }$ as a subset of $Z_{\mathrm{T}}$, is open and closed in $Y_{Z}^{\natural}$, and $Y_{i, Z_{[\sigma]}}$ coincides with the pullback of $Y_{i, Z}^{\natural}$, for each $i$, because $Y_{1, Z}^{\natural}$ and $Y_{2, Z}^{\natural}$ do not overlap either. So $Y_{1}^{\text {min }}$ is also open and closed in $Y^{\text {min }}$. In $Y_{Z_{[\sigma]}}\left(\right.$ respectively $Y_{Z}^{\natural}$ ), let us equip $Y_{i, Z_{[\sigma]}}$ (respectively $\left.Y_{i, Z}^{\natural}\right)$ with its 
reduced subscheme structure in the case of subsets, and with the canonical open subscheme structure in the case of subschemes. Then the canonical isomorphism (2.1.5) induces an isomorphism $Y_{\mathfrak{X}_{\sigma}^{\circ}}^{\natural} \times_{Y_{Z}^{\natural}} Y_{i, Z}^{\natural} \stackrel{\sim}{\rightarrow}\left(Y_{i, \Sigma}^{\text {tor }}\right)_{\bigcup_{\tau \in \Sigma_{Z}^{+}, \bar{\tau} \subset \bar{\sigma}}}^{\wedge} Y_{i, Z_{[\tau]}}$. Thus, for each $W$ as in Proposition 2.1.3, the pullback of $Y_{1}$ to $\left(W^{0}\right)_{\mathrm{T}}$ coincides with the pullback of $Y_{1, Z}^{\natural}$. Hence, $Y_{1}$ is well positioned, with associated $Y_{1}^{\natural}:=\left\{Y_{1, Z}^{\natural}\right\}_{Z}$ as in Definition 2.2.1, as desired.

Proposition 2.3.12. If $\mathrm{Y}$ is a well-positioned subset of $\left(\mathrm{X}_{\mathcal{H}}\right)_{\mathrm{T}}$, and if $\mathrm{Y}_{C}^{\natural} \rightarrow \mathrm{Y}_{\mathrm{Z}}^{\natural}$ is flat and has irreducible fibers for all $\mathrm{Z}$, then the irreducible components of $\mathrm{Y}$ are well-positioned subsets, and their closures in $\mathrm{Y}^{\min }$ and $\mathrm{Y}_{\Sigma}^{\text {tor }}$ are also irreducible components of these partial compactifications.

Proof. Let $\mathrm{Y}_{1}$ be an irreducible component of $\mathrm{Y}$. By definition, its closures in $\mathrm{Y}^{\min }$ and $\mathrm{Y}_{\Sigma}^{\text {tor }}$, respectively, are irreducible components of these partial compactifications. It remains to show that $\mathrm{Y}_{1}$ is well positioned. Let $\bar{U} \rightarrow \mathrm{Y}_{\Sigma}^{\text {tor }}$ and $\bar{U} \rightarrow Y_{\Xi(\sigma)}^{\natural}$ be étale morphisms as in (7) of Theorem 2.3.2, whose pullbacks to $Y_{Z_{[\sigma]}}$ and to $Y_{\Xi_{\sigma}}^{\natural}$ are both open immersions. By the construction of $\bar{U}$ in the proof there, we may and we shall assume that it is an approximation of the pullback of $Y$ to some affine formal scheme $\mathfrak{W}=\operatorname{Spf}(R)$ as in Proposition 2.1.3, with associated affine scheme $W=\operatorname{Spec}(R)$. Up to replacing $\bar{U}$ with an open subscheme, we may and we shall assume that the étale morphisms $\bar{U} \rightarrow \mathrm{Y}_{\Sigma}^{\text {tor }}$ and $\bar{U} \rightarrow Y_{E(\sigma)}^{\natural}$ have irreducible fibers. By [21, IV-2, 2.3.10], the pullback $\bar{U}_{1}$ of $Y_{1}$ to $\bar{U}$ is either empty or an irreducible component of $\bar{U}$. Suppose $\bar{U}_{1}$ is nonempty, with generic point $\eta_{\bar{U}_{1}}$, which is maximal among points of $\bar{U}_{1}$. Since the morphisms $\bar{U} \rightarrow Y_{E(\sigma)}^{\natural} \rightarrow Y_{C}^{\natural} \rightarrow Y_{Z}^{\natural}$ are flat and have irreducible fibers (by assumption), by [21, IV-2, 2.3.10] again, the image $\eta_{Z}$ of $\eta_{\bar{U}_{1}}$ in $Y_{Z}^{\natural}$ is maximal among points of $Y_{Z}^{\natural}$, whose closure $\overline{\left\{\eta_{Z}\right\}}$ in $Y_{Z}^{\natural}$ is an irreducible component, and $\bar{U}_{1}$ coincides with the pullback of $\overline{\left\{\eta_{Z}\right\}}$. Since $Y_{C}^{\natural} \rightarrow Y_{Z}^{\natural}$ has irreducible and hence connected fibers, $\oint_{Y, \Sigma}: Y_{\Sigma}^{\text {tor }} \rightarrow Y^{\text {min }}$ has connected fibers over $Y_{Z}$. Hence, over each connected component of $Y_{Z}^{\natural}$, there is at most one irreducible component of the form $\overline{\left\{\eta_{\mathrm{Z}}\right\}}$ as above. Let $\mathrm{Y}_{1, \mathrm{Z}}^{\natural}$ be the (disjoint) union of such irreducible components. Then its pullback to any $W$ (associated with $\mathfrak{W}$ ) as above coincides with the pullback of $\overline{\left\{\eta_{Z}\right\}}$, and hence with the pullback of $\bar{U}_{1}$, or rather of $Y_{1}$. Since the affine formal schemes $\mathfrak{W}$ as above form an open covering of $\mathfrak{X}_{\Sigma_{Z}}$, for each $Z$, it follows from Lemma 2.2.2 that $Y_{1}$ is well positioned, as desired.

By the same arguments as in the proofs of [38, Propositions 14.1 and 14.2] and [43, Corollary 3.4.15], using the regularity of $W \rightarrow \mathrm{X}_{\mathcal{H}}$ and $W \rightarrow \Xi(\sigma)$ (see [21, IV-2, 6.8.1 and 7.8.3(v)]) for each $W$ as in Proposition 2.1.3, and using the 
facts that $\Xi(\sigma) \rightarrow C$ is surjective and smooth (under the assumption in (4) of Proposition 2.1.2 that $\Sigma$ is smooth), and that $\Xi$ is fiberwise dense in $\Xi(\sigma)$ over $C$, we obtain the following for any well-positioned subset or subscheme $\mathrm{Y}$ of $\left(\mathrm{X}_{\mathcal{H}}\right)_{\mathrm{T}}$ as in Definition 2.2.1, where we equipped $\mathrm{Y}$ with the canonical reduced subscheme structure when $Y$ is only given as a subset, with partial toroidal compactification $Y_{\Sigma}^{\text {tor }}$ as in Definition 2.3.1:

Proposition 2.3.13 (Cf. [38, Proposition 14.1]). Under the assumption (in (4) of Proposition 2.1.2) that $\Sigma$ is smooth, $\mathrm{Y}$ is reduced (respectively normal, respectively regular, respectively Cohen-Macaulay, respectively $\left(R_{i}\right)$, respectively $\left(S_{i}\right)$, one property for each $i \geqslant 0$, respectively flat over $\mathrm{T}$, respectively faithfully flat over $\mathrm{T}$ ) if and only if $\mathrm{Y}_{\Sigma}^{\mathrm{tor}}$ is.

Proposition 2.3.14 (Cf. [38, Proposition 14.2]). Let $P$ be the property of being one of the following: reduced, geometrically reduced, normal, geometrically normal, regular, geometrically regular, Cohen-Macaulay, $\left(R_{i}\right)$, geometric $\left(R_{i}\right)$, and $\left(S_{i}\right)$, one property for each $i \geqslant 0$ (see [21, IV-2, 5.7.2 and 5.8.2]). Under the assumption (in (4) of Proposition 2.1.2) that $\Sigma$ is smooth, the fiber of $\mathrm{Y}_{\Sigma}^{\text {tor }} \rightarrow \mathrm{T}$ over some point $t$ of $\mathrm{T}$ satisfies property $P$ if and only if the corresponding fiber of the open subscheme $\mathrm{Y} \rightarrow \mathrm{T}$ over $t$ does.

However, note that the analogues of Propositions 2.3.13 and 2.3.14 for $\mathrm{Y}^{\mathrm{min}}$ are not true in general. (They are already not true for $\mathrm{X}_{\mathcal{H}}^{\min }$ in general.)

Corollary 2.3.15 (Cf. [38, Corollary 14.4] and [43, Corollary 3.4.15]). Suppose that $\mathrm{Y} \rightarrow \mathrm{T}$ is flat, that its geometric fibers are reduced (respectively have integral local rings), and that $\mathrm{Y}$ is closed in $\left(\mathrm{X}_{\mathcal{H}}\right)_{\mathrm{T}}$. Then all geometric fibers of $\mathrm{Y}_{\Sigma}^{\text {tor }} \rightarrow \mathrm{T}$ have the same number of connected (respectively irreducible) components, and the same is true for $\mathrm{Y} \rightarrow \mathrm{T}$.

The following technical result will be useful in Section 4.5: (The readers may skip it for now, and come back only when reading the proof of Lemma 4.5.24.)

Lemma 2.3.16. Suppose we are in Cases $(\mathrm{Sm}),(\mathrm{Nm})$, and $(\mathrm{Spl})$. Suppose $\mathrm{Y}$ is a well-positioned subset of $\left(\mathrm{X}_{\mathcal{H}}\right)_{\mathrm{T}}$, and suppose $\mathrm{Y}^{\natural}=\left\{\mathrm{Y}_{\mathrm{Z}}^{\natural}\right\}_{\mathrm{Z}}$ is associated with $Y$ as in Definition 2.2.1. For each stratum $Z$ of $X_{\mathcal{H}}^{\min }$, since it is an analogue of $\mathrm{X}_{\mathcal{H}}$, we can define $\mathrm{Z}^{\mathrm{min}}$ and $\mathrm{Y}_{\mathrm{Z}}^{\mathrm{min}}$ as in the case of $\mathrm{X}_{\mathcal{H}}^{\min }$ and $\mathrm{Y}^{\mathrm{min}}$. Let $\overline{\mathrm{Z}}$ denote the closure of $\mathrm{Z}$ in $\mathrm{X}_{\mathcal{H}}^{\min }$, and let $\bar{Y}_{\mathrm{Z}} \subset \bar{Z}_{\mathrm{T}}$ be defined by $\mathrm{Y}_{\mathrm{Z}}$ as in the case of $\mathrm{Y}^{\mathrm{min}} \subset\left(\mathrm{X}_{\mathcal{H}}^{\min }\right)_{\mathrm{T}}$ in Definition 2.3.1. Then the identity morphism on $\mathrm{Z}$ extends to a 
canonical isomorphism $\mathrm{Z}^{\mathrm{min}} \stackrel{\sim}{\rightarrow} \overline{\mathrm{Z}}$. Moreover, $\mathrm{Y}_{\mathrm{Z}}$ is a well-positioned subset of $\mathrm{Z}_{\mathrm{T}}$, and the isomorphism $\mathrm{Z}^{\min } \stackrel{\sim}{\rightarrow} \overline{\mathrm{Z}}$ induces a canonical isomorphism $\mathrm{Y}_{\mathrm{Z}}^{\min } \stackrel{\sim}{\rightarrow} \overline{\mathrm{Y}}_{\mathrm{Z}}$.

Proof. We can also define $Z^{\text {tor }}$ and $Y_{Z}^{\text {tor }}$ (for some collection of cone decompositions). Since we are in Cases $(\mathrm{Sm}),(\mathrm{Nm})$, and $(\mathrm{Spl})$, by the same argument as in the proof of [45, Proposition 4.2], up to replacing $\Sigma$ with a refinement, there exists some top-dimensional $\sigma \in \Sigma_{Z}^{+}$such that the canonical morphism $Z_{[\sigma]} \cong C \rightarrow Z$ extends to a morphism from the closure $Z_{[\sigma]}^{\text {tor }}$ of $Z_{[\sigma]}$ in $X_{\mathcal{H}, \Sigma}^{\text {tor }}$ to $Z^{\text {tor }}$. Moreover, the description of formal charts there shows that $Y_{Z}=Y_{Z}^{\natural}$ is a well-positioned subset of $Z_{T}$, with associated collection $\left(Y_{Z}\right)^{\natural}=\left\{\left(Y_{Z}\right)_{Z^{\prime}}^{\natural}\right\}_{Z^{\prime}}$ indexed by the strata $Z^{\prime}$ of $X^{\min }$ contained in $\bar{Z}$. By taking any $\omega_{X_{\mathcal{H}}^{\min }}$ as in (2) of Theorem 2.3.2 such that its pullback to $Z_{[\sigma]}^{\text {tor }}$ descends to an ample invertible sheaf over $Z^{\text {min }}$, the canonical morphism $Z_{[\sigma]}^{\text {tor }} \rightarrow X_{\mathcal{H}}^{\text {min }}$ induces a canonical morphism $Z^{\text {min }} \rightarrow \bar{Z}$, which in turn induces a canonical morphism $Y_{Z}^{\min } \rightarrow \bar{Y}_{Z}$. (Note that there is at most one morphism $Z^{\min } \rightarrow \bar{Z}$ extending the identity morphism on the open dense subscheme $Z$ of the noetherian normal scheme $Z^{\mathrm{min}}$.) For each stratum $Z^{\prime}$ of $X_{\mathcal{H}}^{\min }$ contained in $\bar{Z}$, and for each $\tau^{\prime} \in \Sigma_{Z^{\prime}}^{+}$, the composition $Z_{\left[\tau^{\prime}\right]}^{\prime} \rightarrow Z^{\prime} \rightarrow \bar{Z}$ factors through $Z^{\prime} \rightarrow Z^{\text {min }} \rightarrow \bar{Z}$. Therefore, $Z^{\text {min }} \rightarrow \bar{Z}$ induces a bijection between geometric points, and even induces the identity morphism from $Z^{\prime}$ as a stratum of $Z^{\text {min }}$ to $Z^{\prime}$ as a stratum of $X_{\mathcal{H}}^{\min }$. Since $Y_{Z^{\prime}}^{\natural}$ and $Y_{Z^{\prime}}$ coincide as subsets of $Z_{T}^{\prime}$, for each $Z^{\prime}$, if $Z^{\min } \rightarrow \bar{Z}$ is an isomorphism, then the induced morphism $Y_{Z}^{\min } \rightarrow \bar{Y}_{Z}$ is also an isomorphism.

It remains to show that the canonical morphism $Z^{\min } \rightarrow \bar{Z}$ is an isomorphism. By the same strategy as in [19, Ch. V, page 152], it suffices to show that it induces isomorphisms between completions of strict local rings. Let $Z^{\prime}$ be any stratum of $X^{\min }$ contained in $\bar{Z}$. For the sake of clarity, we shall denote $Z^{\prime}$ as $Z^{\prime \prime}$ when we view it as a stratum of $Z^{\text {min }}$, and denote with superscripts (respectively ") various objects of $X_{\mathcal{H}}^{\min }$ (respectively $Z^{\text {min }}$ ) that are associated with $Z^{\prime}$ (respectively $Z^{\prime \prime}$ ). The morphism $Z_{[\sigma]}^{\text {tor }} \rightarrow X_{\mathcal{H}}^{\min }$ that induced $Z^{\min } \rightarrow \bar{Z}$ also induces a proper surjective morphism $C^{\prime} \rightarrow C^{\prime \prime}$ between noetherian normal schemes, and an injective homomorphism $\mathbf{S}^{\prime \prime} \rightarrow \mathbf{S}^{\prime}$ such that the pullback of $\Psi^{\prime \prime}\left(\ell^{\prime \prime}\right)$ under $C^{\prime} \rightarrow C^{\prime \prime}$ is canonically isomorphic to $\Psi^{\prime}\left(\ell^{\prime}\right)$ when $\ell^{\prime}$ is the image of $\ell^{\prime \prime}$ under $\mathbf{S}^{\prime \prime} \rightarrow \mathbf{S}^{\prime}$, so that we have canonically induced injective morphisms

$$
\underline{\mathrm{FJ}}^{\prime \prime},\left(\ell^{\prime \prime}\right):=\left(C^{\prime \prime} \rightarrow \mathrm{Z}^{\prime \prime}\right)_{*} \Psi^{\prime \prime}\left(\ell^{\prime \prime}\right) \rightarrow \underline{\mathrm{FJ}}^{\prime,\left(\ell^{\prime}\right)}:=\left(C^{\prime} \rightarrow \mathrm{Z}^{\prime}\right)_{*} \Psi^{\prime}\left(\ell^{\prime}\right) .
$$

By [36, Proposition 7.2.3.16, and the errata] in Case (Sm), [38, Proposition 12.13] in Case (Nm), and [43, Proposition 4.2.20] in Case (Spl), for each geometric point $\bar{x}$ of $Z^{\prime}$, which we also view as geometric points of $Z^{\min }$ and $\bar{Z}$, we have ring 
homomorphisms

$$
\begin{aligned}
&\left(\mathscr{O}_{\overline{\mathbf{Z}}}\right)_{\bar{x}}^{\wedge} \stackrel{\text { can. }}{\rightarrow}\left(\mathscr{O}_{\mathbf{Z}^{\min }}\right)_{\bar{x}}^{\wedge} \cong\left(\prod_{\ell^{\prime \prime} \in\left(\mathbf{P}^{\prime \prime}\right)^{\vee}}\left(\underline{\mathrm{FJ}}^{\prime \prime},\left(\ell^{\prime \prime}\right)\right)_{\bar{x}}^{\wedge}\right)^{\Gamma^{\prime \prime}} \\
& \rightarrow\left(\prod_{\ell^{\prime} \in\left(\mathbf{P}^{\prime}\right)^{\vee} \cap \operatorname{ker}\left(\mathbf{S}^{\prime} \rightarrow \mathbf{S}\right)}\left(\underline{\mathrm{FJ}}^{\prime,\left(\ell^{\prime}\right)}\right)_{\bar{x}}^{\wedge}\right)^{\operatorname{Stab}_{\left(\mathbf{P}^{\prime}\right) \vee \cap \operatorname{ker}\left(\mathbf{S}^{\prime} \rightarrow \mathbf{S}\right)}\left(\Gamma^{\prime}\right)},
\end{aligned}
$$

where $(\cdot)_{\bar{x}}^{\wedge}$ denotes the pullbacks of various objects $(\cdot)$ over $Z^{\prime}$ to the completion of the strict local ring of $Z^{\prime}$ at $\bar{x}$, where the first homomorphism is injective because $\bar{Z}$ is reduced and $Z^{\text {min }} \rightarrow \bar{Z}$ is surjective, where the third homomorphism is defined and injective by the explanation in the previous sentence, and where the composition of all homomorphisms in (2.3.17) is an isomorphism. But then all homomorphisms in (2.3.17) are isomorphisms, because they are already known to be injective. Thus, $Z^{\mathrm{min}} \rightarrow \bar{Z}$ is an isomorphism, because $Z^{\prime}$ and $\bar{x}$ are arbitrary, as desired.

\subsection{Functorial properties and Hecke actions.}

PROPOSITION 2.4.1. Under any morphism $\mathrm{T}^{\prime} \rightarrow \mathrm{T}$ of locally noetherian schemes over $\mathrm{S}$, the pullback

$$
\mathrm{Y}^{\prime}:=\mathrm{Y} \times_{\mathrm{T}} \mathrm{T}^{\prime}
$$

of a well-positioned subset (respectively subscheme) $\mathrm{Y}$ of $\left(\mathrm{X}_{\mathcal{H}}\right)_{\mathrm{T}}$ is a wellpositioned subset (respectively subscheme) of $\left(\mathrm{X}_{\mathcal{H}}\right)_{\mathrm{T}^{\prime}}$. If $\mathrm{Y}^{\natural}=\left\{\mathrm{Y}_{Z}^{\natural}\right\}_{\mathrm{Z}}$ is associated with $\mathrm{Y}$, then

$$
\mathrm{Y}^{\prime, \natural}:=\left\{\mathrm{Y}_{\mathrm{Z}}^{\natural} \times{ }_{\mathrm{T}} \mathrm{T}^{\prime}\right\}
$$

is associated with $\mathrm{Y}^{\prime}$, as in Definition 2.2.1. Let $\mathrm{Y}^{\prime, \min }$ and $\mathrm{Y}_{\Sigma}^{\prime \text {,tor }}$ denote the partial minimal and toroidal compactifications of $\mathrm{Y}^{\prime}$, respectively, as in Definition 2.3.1. Then the canonical morphisms $\mathrm{Y}^{\prime}$, min $\rightarrow \mathrm{Y}^{\text {min }} \times_{\mathrm{T}} \mathrm{T}^{\prime}$ and $\mathrm{Y}_{\Sigma}^{\prime, \text { tor }} \rightarrow \mathrm{Y}_{\Sigma}^{\text {tor }} \times_{\mathrm{T}} \mathrm{T}^{\prime}$ induce isomorphisms between the reduced subschemes. In the case of subschemes, the latter morphism $\mathrm{Y}_{\Sigma}^{\prime \text {,tor }} \rightarrow \mathrm{Y}_{\Sigma}^{\text {tor }} \mathrm{X}_{\mathrm{T}} \mathrm{T}^{\prime}$ is an isomorphism, without having to pass to the morphism between reduced subschemes.

Proof. For each $Z$, let $Y_{Z}^{\natural}$ be associated with $Y$ as in Definition 2.2.1, and let $Y_{C}^{\natural}$ denote its pullback under $C \rightarrow Z$. Then $Y^{\prime}$ is a well-positioned subset (respectively subscheme) because the pullback of $Y^{\prime}$ to $\left(W^{0}\right)_{T^{\prime}}$ coincides with the pullback of $Y_{C}^{\prime, \natural}:=Y_{C}^{\natural} \times_{T} T^{\prime}$, which is in turn the pullback of $Y_{Z}^{\prime, \natural}:=Y_{Z}^{\natural} \times_{T} T^{\prime}$, as subsets (respectively subschemes), for each $W$ as in Proposition 2.1.3. As in the proof of Theorem 2.3.2, by the flatness of $W \rightarrow \mathrm{X}_{\mathcal{H}, \Sigma}^{\text {tor }}$ and $W \rightarrow \Xi(\sigma)$, 
the pullback of $\mathrm{Y}_{\Sigma}^{\prime \text {,tor }}$ to $W_{\mathrm{T}^{\prime}}$ coincides with the pullback of $\mathrm{Y}_{C}^{\prime, \text {, }}$, which also coincides with the pullback of $Y_{\Sigma}^{\text {tor }}$, as a subset (respectively subscheme). The remaining assertions then follow from the definitions.

PROPOSITION 2.4.2. If $\mathrm{Y}$ is a well-positioned subset (respectively subscheme) of $\left(\mathrm{X}_{\mathcal{H}}\right)_{\mathrm{T}}$, if $\left(\mathrm{X}_{\mathcal{H}^{\prime}}\right)_{\mathrm{T}} \rightarrow\left(\mathrm{X}_{\mathcal{H}}\right)_{\mathrm{T}}$ is defined by an inclusion $\mathcal{H}^{\prime} \subset \mathcal{H}$ of open compact subgroups of $\mathrm{G}(\hat{\mathbb{Z}})$ (and other data), then the preimage $\mathrm{Y}^{\prime}$ of $\mathrm{Y}$ in $\left(\mathrm{X}_{\mathcal{H}^{\prime}}\right)_{\mathrm{T}}$ is also a well-positioned subset (respectively subscheme). If $\mathrm{Y}^{\natural}=\left\{Y_{Z}^{\natural}\right\}_{Z}$ is associated with $\mathrm{Y}$, and if $\mathrm{Y}_{\mathrm{Z}^{\prime}}^{\prime,}$ is the pullback of $\mathrm{Y}_{\mathbf{Z}}^{\natural}$ under $\mathrm{Z}^{\prime} \rightarrow \mathrm{Z}$, for each stratum $\mathrm{Z}^{\prime}$ of $\mathrm{X}_{\mathcal{H}^{\prime}}^{\min }$ above a stratum $\mathrm{Z}$ of $\mathrm{X}_{\mathcal{H}}^{\min }$, then $\mathrm{Y}^{\prime, \natural}:=\left\{\mathrm{Y}_{\mathbf{Z}^{\prime}}^{\prime, \natural}\right\}$ is associated with $\mathrm{Y}^{\prime}$, as in Definition 2.2.1. Let $\mathrm{Y}^{\prime, \min }$ and $\mathrm{Y}_{\Sigma^{\prime}}^{\prime \text { tor }}$ denote the partial compactifications of $\mathrm{Y}^{\prime}$, respectively, as in Definition 2.3.1. Then the canonical morphisms $\mathrm{Y}^{\prime}$,min $\rightarrow$ $\mathrm{Y}^{\text {min }} \times_{\mathrm{X}_{\mathcal{H}}^{\text {min }}} \mathrm{X}_{\mathcal{H}^{\prime}}^{\text {min }}$ and $\mathrm{Y}_{\Sigma^{\prime}}^{\prime \text {,tor }} \rightarrow \mathrm{Y}_{\Sigma}^{\text {tor }} \times_{\mathrm{X}_{\mathcal{H}, \Sigma}^{\text {tor }}} \mathrm{X}_{\mathcal{H}^{\prime}, \Sigma^{\prime}}^{\text {tor }}$ induce isomorphisms between the reduced subschemes. In the case of subschemes, the latter morphism between partial toroidal compactifications is an isomorphism by itself.

Proof. By [36, Proposition 6.4.3.4] in Case (Sm), by [41, Proposition 7.1] in Case (Nm), by [43, Proposition 3.4.10] in Case ( $\mathrm{Spl}$ ), and by [50, Sections 4.1.12 and 5.2.12] and the same facts used in the proof of Proposition 2.1.3 in Case $(\mathrm{Hdg})$, we have a proper morphism $\mathrm{X}_{\mathcal{H}^{\prime}, \Sigma^{\prime}}^{\text {tor }} \rightarrow \mathrm{X}_{\mathcal{H}, \Sigma}^{\text {tor }}$ for some $\Sigma^{\prime}$ refining $\Sigma$, which induces a proper morphism $\left(\mathbf{X}_{\mathcal{H}^{\prime}, \Sigma^{\prime}}^{\text {tor }}\right)_{\bigcup_{\left[\sigma^{\prime}\right] \in \Sigma_{Z^{\prime}}^{\prime} / \Gamma^{\prime}} \mathrm{Z}_{\left[\sigma^{\prime}\right]}^{\prime}}^{\wedge} \rightarrow\left(\mathbf{X}_{\mathcal{H}, \Sigma}^{\text {tor }}\right)_{\bigcup_{[\sigma] \in \Sigma_{Z}^{+} / \Gamma}^{+}} \mathrm{Z}_{[\sigma]}$ between the formal completions (where any object denoted with a prime means the analogous object at level $\mathcal{H}^{\prime}$ ). This proper morphism is compatible with the proper morphism $\Xi(\sigma)^{\prime}:=\bigcup_{\tau \in \Sigma_{Z}^{\prime \prime}, \tau \subset \sigma} \Xi^{\prime}(\tau) \rightarrow \Xi(\sigma)$ extending $\Xi^{\prime} \rightarrow \Xi$ and covering $C^{\prime} \rightarrow C$ and $Z^{\prime} \rightarrow Z$. For each affine open formal subscheme $\mathfrak{W}=\operatorname{Spf}(R)$ of $\mathfrak{X}_{\sigma}^{\circ}$, which induces a canonical morphism $W=\operatorname{Spec}(R) \rightarrow \Xi(\sigma)$, its pullback under $\Xi(\sigma)^{\prime} \rightarrow \Xi(\sigma)$ is covered by finitely many $W_{i}=\operatorname{Spec}\left(R_{i}\right)$, where $\operatorname{Spec}\left(R_{i}\right) \rightarrow \Xi(\sigma)^{\prime}$ is induced by some affine open formal subscheme $\mathfrak{W}_{i}=\operatorname{Spf}\left(R_{i}\right)$ of $\mathfrak{X}_{\tau_{i}}^{\prime \prime \circ}$, for some $\tau_{i} \in \Sigma_{Z}^{\prime \prime+}$ such that $\tau_{i} \subset \sigma$.

Now suppose $Y$ is a well-positioned subset (respectively subscheme), with associated $Y^{\natural}=\left\{Y_{Z}^{\natural}\right\}_{Z}$ as in Definition 2.2.1. Let us denote by $Y^{\prime}$ the pullback of $Y$ to $\left(X_{\mathcal{H}^{\prime}}\right)_{\mathrm{T}}$, and by $Y_{Z^{\prime}}^{\prime, \natural}$ the pullback of $Y_{Z}^{\natural}$ to $Z^{\prime}$. Since the pullback of $Y$ to $\left(W^{0}\right)_{\mathrm{T}}$ coincides with the pullback of $Y_{Z}^{\natural}$, since $\Xi(\sigma)^{\prime} \rightarrow \Xi(\sigma)$ extends the canonical morphism $\Xi^{\prime} \rightarrow \Xi$, and since the two compositions $\Xi^{\prime} \rightarrow Z^{\prime} \rightarrow Z$ and $\Xi^{\prime} \rightarrow \Xi \rightarrow Z$ coincide, the pullback of $Y$ to $\left(W_{i}^{0}\right)_{\mathrm{T}}$ coincides with the pullback of $Y_{Z^{\prime}}^{\prime, \natural}$ as a subset (respectively subscheme). Since $W$ is arbitrary, $Y^{\prime}$ is also a wellpositioned subset (respectively subscheme), with associated $Y^{\prime, \natural}:=\left\{Y_{Z^{\prime}}^{\prime, \natural}\right\}$ as in Definition 2.2.1. As in the proof of Theorem 2.3.2, by the flatness of $W \rightarrow \mathrm{X}_{\mathcal{H}, \Sigma}^{\text {tor }}$, 
$W \rightarrow \Xi(\sigma), W_{i} \rightarrow X_{\mathcal{H}^{\prime}, \Sigma^{\prime}}^{\text {tor }}$ and $W_{i} \rightarrow \Xi(\sigma)^{\prime}$, the pullback of $Y_{\Sigma}^{\text {tor }}$ to $W_{\mathrm{T}}$ coincides with the pullback of $Y_{Z}^{\natural}$, and the pullback of $Y_{\Sigma^{\prime}}^{\prime, \text { tor }}$ to $\left(W_{i}\right)_{\mathrm{T}}$ coincides with the pullback of $Y_{Z^{\prime}}^{\prime, \natural}$, and so the pullback of $Y_{\Sigma^{\prime}}^{\prime \text {,tor }}$ to $\left(W_{i}\right)_{\mathrm{T}}$ coincides with the pullback of $Y_{\Sigma}^{\text {tor }}$, as subsets (respectively subschemes), for each $i$. The remaining assertions then follow from the definitions.

For many arithmetic applications, it is desirable to have the following:

Proposition 2.4.3 (Cf. [38, Propositions 13.7, 13.9, and 13.15] and [41, Propositions 7.3 and 7.5]). Suppose that $\mathcal{H}$ and $\mathcal{H}^{\prime}$ are two open compact subgroups of $\mathrm{G}(\hat{\mathbb{Z}})$, that $g \in \mathrm{G}\left(\mathbb{A}^{\infty}\right)$, and that $\Sigma^{\prime}$ is a g-refinement of $\Sigma$ as in [36, Definition 6.4.3.3], such that $\mathcal{H}^{\prime} \subset g \mathcal{H}^{-1}$, and such that the morphisms

$$
\begin{gathered}
{[g]: \mathbf{X}_{\mathcal{H}^{\prime}} \rightarrow \mathbf{X}_{\mathcal{H}},} \\
{[g]^{\min }: \mathbf{X}_{\mathcal{H}^{\prime}}^{\min } \rightarrow \mathbf{X}_{\mathcal{H}}^{\min },}
\end{gathered}
$$

and

$$
[g]^{\text {tor }}: X_{\mathcal{H}^{\prime}, \Sigma^{\prime}}^{\text {tor }} \rightarrow \mathrm{X}_{\mathcal{H}, \Sigma}^{\text {tor }}
$$

are compatibly defined. (See [36, Propositions 6.4.3.4 and 7.2.5.1] in Case (Sm); see [38, Propositions 13.7, 13.9, and 13.15] and [41, Proposition 7.3] in Case $(\mathrm{Nm})$; see [43, Propositions 2.4.17, 3.4.10, and 4.3.11] in Case ( $\mathrm{Spl})$; and see [50, Sections 4.1.12 and 5.2.12] in Case (Hdg).)

Suppose that $\mathrm{T}^{\prime} \rightarrow \mathrm{T}$ is a morphism over $\mathrm{S}$, that $\mathrm{Y}$ is a well-positioned subset (respectively subscheme) of $\left(\mathrm{X}_{\mathcal{H}}\right)_{\mathrm{T}}$, and that $\mathrm{Y}^{\prime}$ is a well-positioned subset (respectively subscheme) of $\left(\mathrm{X}_{\mathcal{H}^{\prime}}\right)_{T^{\prime}}$, such that the morphism (2.4.4) induces a morphism

$$
[g]: Y^{\prime} \rightarrow Y
$$

of sets (respectively schemes). Then the morphism (2.4.5) induces a morphism

$$
[g]^{\min }: \mathrm{Y}^{\prime, \min } \rightarrow \mathrm{Y}^{\min }
$$

extending (2.4.7), and the morphism (2.4.6) induces a morphism

$$
[g]^{\text {tor }}: Y_{\Sigma^{\prime}}^{\prime \text { tor }} \rightarrow Y_{\Sigma}^{\text {tor }}
$$

extending (2.4.7) and compatible with (2.4.8) under the canonical morphisms $\oint_{\mathcal{H}^{\prime}, \Sigma^{\prime}}: \mathrm{Y}_{\Sigma^{\prime}}^{\prime \text { tor }} \rightarrow \mathrm{Y}^{\prime, \min }$ and $\oint_{\mathcal{H}, \Sigma}: \mathrm{Y}_{\Sigma}^{\text {tor }} \rightarrow \mathrm{Y}^{\text {min }}$ as in (1) of Theorem 2.3.2.

Suppose moreover that $\mathrm{Y}^{\prime}$ coincides with the pullback of $\mathrm{Y}$ under (2.4.4) as a well-positioned subset (respectively subscheme). Then the canonical morphisms

$$
\mathrm{Y}^{\prime, \text { min }} \rightarrow \mathrm{Y}^{\min } \times_{\left.\mathrm{X}_{\mathcal{H}}^{\min },[g]\right]^{\min }} \mathrm{X}_{\mathcal{H}^{\prime}}^{\min }
$$


and

$$
\mathrm{Y}_{\Sigma^{\prime}}^{\prime \text { tor }} \rightarrow \mathrm{Y}_{\Sigma}^{\text {tor }} \times_{\mathrm{X}_{\mathcal{H}, \Sigma}^{\text {tor }},[g]^{\text {tor }}} \mathrm{X}_{\mathcal{H}^{\prime}, \Sigma^{\prime}}^{\text {tor }}
$$

induce isomorphisms between the reduced subschemes. In the case of subschemes, the latter morphism between partial toroidal compactifications is an isomorphism by itself. If $\mathrm{Y}^{\natural}=\left\{Y_{Z}\right\}_{Z}$ is associated with $\mathrm{Y}$ as in Definition 2.2.1, and if $\mathrm{Y}_{Z^{\prime}}^{\prime, \natural}$ is the pullback of $Y_{Z}^{\natural}$ under $Z^{\prime} \rightarrow Z$, for each stratum $Z^{\prime}$ of $X_{\mathcal{H}^{\prime}}^{\min }$ that is mapped to a stratum $\mathrm{Z}$ of $\mathrm{X}_{\mathcal{H}}^{\min }$ under (2.4.5), then $\mathrm{Y}^{\prime, \natural}:=\left\{\mathrm{Y}_{\mathrm{Z}^{\prime}}^{\prime,}\right\}$ is associated with $\mathrm{Y}^{\prime}$, as in Definition 2.2.1.

Proof. These follow from the definitions, from the constructions and properties of (2.4.4), (2.4.5), and (2.4.6) in the references mentioned, and from the same arguments as in the proofs of Propositions 2.4.1 and 2.4.2.

\subsection{Vanishing of higher direct images, and Koecher's principle. For} simplicity, let us assume that $\mathrm{T}=\operatorname{Spec}\left(R_{1}\right)$ is some noetherian affine scheme over $\mathrm{S}=\operatorname{Spec}\left(R_{0}\right)$. Let $\mathrm{Y}$ be a well-positioned subset or subscheme of $\left(\mathrm{X}_{\mathcal{H}}\right)_{\mathrm{T}}$, with associated collection $Y^{\natural}=\left\{Y_{Z}^{\natural}\right\}_{Z}$ as in Definition 2.2.1, and with partial minimal and toroidal compactifications $Y^{\text {min }}$ and $Y_{\Sigma}^{\text {tor }}$ as in Definition 2.3.1 and Theorem 2.3.2. Let

$$
h: C \rightarrow \mathrm{Z}
$$

denote the structural morphism, with induced morphism

$$
h_{Y}: Y_{C}^{\natural} \rightarrow Y_{Z}^{\natural} \text {. }
$$

(Recall that, in the case of subschemes, this is exactly the pullback of $h$; but in the case of subsets, this is just the induced map between reduced subschemes.) For each $\ell \in \mathbf{S}$, let $\Psi_{Y}(\ell)$ denote the pullback of $\Psi(\ell)$ under $Y_{C}^{\natural} \rightarrow C$. As in [39, Section 6], let

$$
\mathbf{P}^{\vee,+}:=\{\ell \in \mathbf{S}:\langle\ell, y\rangle>0, \forall y \in \mathbf{P}-\{0\}\} .
$$

(See Proposition 2.1.2 for the meaning of $\mathbf{S}$, and so forth.)

LEMMA 2.5.1 (Cf. [41, Lemma 8.1]). There exist infinitely many integers $n$ prime to $p$ such that, for each such $n$, there exists a finite étale commutative group scheme $H_{n}$ of order prime to $p$ over $Z$ acting on $C$ via morphisms compatible with $h: C \rightarrow \mathrm{Z}$, inducing canonical morphisms $C \rightarrow C / H_{n} \stackrel{\sim}{\rightarrow} C$ over $\mathrm{Z}$, whose composition we denote as $[n]$, such that

$$
[n]^{*} \Psi(\ell) \cong \Psi\left(n^{2} \ell\right) \cong \Psi(\ell)^{\otimes n^{2}},
$$

for each $\ell \in \mathbf{S}$. Moreover, for any $R_{0}$-algebra $R$, the canonical morphism

$$
\Psi(\ell) \otimes_{R_{0}} R \rightarrow[n]_{*}\left(\Psi\left(n^{2} \ell\right) \otimes_{R_{0}} R\right)
$$


defined by adjunction identifies the left-hand side with a direct summand of the right-hand side, consisting of $H_{n}$-invariants (cf. [63, page 72, Corollary]).

Proof. In Case (Sm), this follows from the constructions in [36, Sections 6.2.26.2.4; see also the errata], because $R_{0}$ is flat over $\mathcal{O}_{F_{0} \text { ( (口) }}$. In Case $(\mathrm{Nm})$, this follows from [41, Lemma 8.1], because $R_{0}$ is flat over $\mathcal{O}_{F_{0},(p)}$. In Case $(\mathrm{Spl})$, this follows from [43, Lemma 4.4.5], because $R_{0}$ is flat over $\mathcal{O}_{K}$. (See

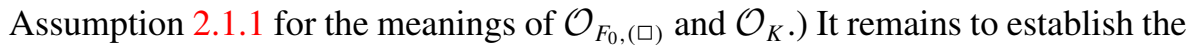
proposition in Case (Hdg). By the constructions in [50, Sections 4.1-4.2], there exists some open compact subgroup $\mathcal{H}^{\prime}$ of $\mathcal{H}$ such that, for some stratum $Z^{\prime}$ at level $\mathcal{H}^{\prime}$ above $\mathbf{Z}$, the corresponding $C^{\prime} \rightarrow Z^{\prime}$ is an abelian scheme, and $C \rightarrow \mathbf{Z}$ is an equivariant quotient of $C^{\prime} \rightarrow Z^{\prime}$ by some finite group $H^{\prime}$. Then there exist infinitely many integers $n$ prime to $p$ and the order of $H^{\prime}$ such that the morphism $[n]: C^{\prime} \rightarrow C^{\prime}$ over $Z^{\prime}$ defined by multiplication by $n$, or equivalently by quotient by the finite étale subgroup scheme $C^{\prime}[n]$ of $n$-torsion points of $C^{\prime}$, descends to a morphism $[n]: C \rightarrow C$ over $\mathrm{Z}$ defined by the quotient by some finite étale commutative group scheme $H_{n}$ of order prime to $p$ over $Z$. Moreover, we have the isomorphism (2.5.2) by descent and by its analogue at level $\mathcal{H}^{\prime}$, again by the constructions in [50, Sections 4.1-4.2]. Finally, since the order of $H_{n}$ is prime to $p$ and hence invertible in the base ring $R_{1}$, the assertion for (2.5.3) holds by the same averaging argument as in the proof of [41, Lemma 8.1].

LemMA 2.5.4. The morphisms $C \rightarrow C / H_{n} \stackrel{\sim}{\rightarrow} C$ in Lemma 2.5 .1 induces similar morphisms $\mathrm{Y}_{C}^{\natural} \rightarrow \mathrm{Y}_{C}^{\natural} / H_{n} \stackrel{\sim}{\rightarrow} \mathrm{Y}_{C}^{\natural}$ compatible with $h_{\mathrm{Y}}: \mathrm{Y}_{C}^{\natural} \rightarrow \mathrm{Y}_{\mathrm{Z}}^{\natural}$, whose composition we denote as $[n]_{Y}$, such that

$$
[n]_{Y}^{*} \Psi_{Y}(\ell) \cong \Psi_{Y}\left(n^{2} \ell\right) \cong \Psi_{Y}(\ell)^{\otimes n^{2}},
$$

for each $\ell \in \mathbf{S}$. Moreover, for any $R_{1}$-algebra $R$, the canonical morphism

$$
\Psi_{\mathrm{Y}}(\ell) \otimes_{R_{1}} R \rightarrow[n]_{\mathrm{Y}, *}\left(\Psi_{\mathrm{Y}}\left(n^{2} \ell\right) \otimes_{R_{1}} R\right)
$$

defined by adjunction identifies the left-hand side with a direct summand of the right-hand side, consisting of $H_{n}$-invariants.

Proof. Since the order of $H_{n}$ is invertible in the base ring $R_{1}$, there is a canonical splitting of (2.5.3) defined (by descent, up to étale localizations trivializing $H_{n}$ ) by sending each section $x$ of the right-hand side to the section $\left(\# H_{n}\right)^{-1} \sum_{h \in H_{n}} h(x)$ of the left-hand side defined by averaging, which is compatible with arbitrary base changes. Hence, this lemma follows from Lemma 2.5.1.

By Lemma 2.5.4, and by the same arguments as in the proofs of [41, Propositions 8.3 and 8.4 ], we obtain the following two propositions: 
Proposition 2.5.6 (Cf. [41, Proposition 8.3]). Suppose $\ell \in \mathbf{P}^{\vee,+}$. Then

$$
R^{i} h_{Y_{, *}}\left(\Psi_{\mathrm{Y}}(\ell) \otimes_{R_{1}} R\right)=0
$$

for all $i>0$ and all $R_{1}$-algebra $R$.

Proposition 2.5.7 (Cf. [41, Proposition 8.4]). Suppose that $\mathbf{S} \cong \mathbb{Z}$, that $\ell \in \mathbf{S}$ is negative, and that $h_{\mathrm{Y}}$ has positive-dimensional fibers. Then

$$
h_{\mathrm{Y}, *}\left(\Psi_{\mathrm{Y}}(\ell) \otimes_{R_{1}} R\right)=0
$$

for all $R_{1}$-algebra $R$.

Definition 2.5.8 (Cf. [39, Corollary 5.8] and [41, Definition 8.5]). Let $R$ be an $R_{1}$-algebra. We say that a quasicoherent sheaf $\mathscr{E}$ over $\mathrm{Y}_{\Sigma}^{\text {tor }}$ is formally canonical (respectively formally subcanonical) (over $R$ ) if it satisfies the following condition: Suppose $\bar{x}$ is a geometric point over $Y_{Z}^{\natural}$, for some stratum $\mathrm{Z}$ of $\mathrm{X}_{\mathcal{H}}^{\min }$. In what follows, we shall denote by $(\cdot)_{\bar{x}}^{\wedge}$ the pullback of $(\cdot)$ under $\left(Y_{Z}^{\natural}\right)_{\bar{x}}^{\wedge} \rightarrow Y_{Z}^{\natural}$. Then there exists a quasicoherent sheaf $\mathscr{E}_{0, \bar{x}}$ over $\left(Y_{C}^{\natural}\right)_{\bar{x}}^{\wedge}$ satisfying the following properties:

(1) For each $\sigma \in \Sigma_{Z}^{+}$, the pullback $\mathscr{E}^{\wedge}$ of $\mathscr{E}$ to the affine formal subscheme $\left(\mathrm{Y}_{\mathfrak{X}_{\sigma}^{\natural}}^{\natural}\right)_{\bar{x}}^{\wedge}$ of $\left(\mathrm{Y}_{\mathfrak{X}_{\Sigma_{Z}}^{\natural}}^{\natural}\right)_{\bar{x}}^{\wedge}$ (see (5) of Theorem 2.3.2) is of the form

$$
\hat{\bigoplus}_{\ell \in ?}\left(\left(\Psi_{\mathrm{Y}}(\ell)\right)_{\bar{x}}^{\wedge} \otimes_{\mathscr{O}_{\left(Y_{C}^{\natural}\right) \hat{\bar{x}}}} \mathscr{E}_{0, \bar{x}}\right)
$$

(as an $\mathscr{O}_{\left(\mathrm{Y}_{C}^{\natural}\right)}$-module), where $?=\sigma^{\vee}$ (respectively $?=\sigma_{+}^{\vee}$ ), where $\sigma_{+}^{\vee}$ is the intersection of $\tau_{0}^{\vee}$ (in $\mathbf{S}$ ) for $\tau$ running through faces of $\sigma$ in $\Sigma_{\mathbf{Z}}$ (including $\sigma$ itself).

(2) There is a finite exhaustive filtration on $\mathscr{E}_{0, \bar{x}}$ whose graded pieces are isomorphic to pullbacks (under the structural morphism $\left(\mathrm{Y}_{C}^{\natural}\right)_{\bar{x}}^{\wedge} \rightarrow \mathrm{T}$ ) of quasicoherent sheaves over $\mathrm{T}=\operatorname{Spec}\left(R_{1}\right)$ associated with finite $R$-modules.

REMARK 2.5.9. In Case (Sm), by [39, Corollary 5.8], the pullbacks of the usual canonical (respectively subcanonical) extensions $\mathcal{E}_{\mathrm{M}_{0}}^{\text {can }}(W)$ (respectively $\mathcal{E}_{\mathrm{M}_{0}}^{\mathrm{sub}}(W)$ ) as in [35, Definition 6.13] are formally canonical (respectively subcanonical) as in Definition 2.5.8. The same are true for their pullbacks to Cases $(\mathrm{Nm})$ and $(\mathrm{Spl})$, which is feasible when $\mathrm{G}\left(\mathbb{Z}_{p}\right)$ is a hyperspecial maximal open compact subgroup of $\mathrm{G}\left(\mathbb{Q}_{p}\right)$. 
THEOREM 2.5.10 (Vanishing of higher direct images; cf. [39, Theorem 3.9] and [41, Theorem 8.6]). Suppose that $R$ is an $R_{1}$-algebra, and that $\mathscr{E}$ is a quasicoherent sheaf over $\mathrm{X}_{\mathcal{H}, \Sigma}^{\text {tor }}$ that is formally canonical (respectively formally subcanonical) over $R$, as in Definition 2.5.8. Let $\mathrm{D}^{\prime}$ be the effective Cartier divisor defined over $\mathrm{X}_{\mathcal{H}, \Sigma}^{\text {tor }}$ as in [41, Corollary 6.7] (whose definition also work here), whose support is

$$
\mathrm{D}:=\mathrm{X}_{\mathcal{H}, \Sigma}^{\text {tor }}-\mathrm{X}_{\mathcal{H}}
$$

with its reduced subscheme structure, and let

$$
\mathscr{E}\left(-n \mathrm{D}^{\prime}\right):=\mathscr{E} \otimes_{\mathscr{O}_{\text {Xor }}^{\text {tor }},} \mathscr{O}_{\mathrm{X}_{\mathcal{H}, \Sigma}^{\text {tor }}}\left(-n \mathrm{D}^{\prime}\right),
$$

for each integer $n$. Then

$$
R^{i}\left(\oint_{\mathrm{Y}, \Sigma}\right)_{*} \mathscr{E}\left(-n \mathrm{D}^{\prime}\right)=0
$$

for all $i>0$ and $n>0$ (respectively $n \geqslant 0)$.

Proof. Thanks to Theorem 2.3.2, which provides almost the same axiomatic setup in [39, Section 4], except that $h_{Y}: Y_{C}^{\natural} \rightarrow Y_{Z}^{\natural}$ is in general not an abelian scheme torsor over a finite cover of $Y_{Z}^{\natural}$; and thanks to Proposition 2.5.6, which implies the analogue of [39, Lemma 6.1] for the context here; the same argument as in the proof of [39, Theorem 3.9] also works here.

THEOREM 2.5.11 (Koecher's principle; cf. [39, Theorem 2.3] and [41, Theorem 8.7]). Suppose $\mathcal{O} \otimes_{\mathbb{Z}} \mathbb{Q}$ is a simple algebra over $\mathbb{Q}$. Suppose $R$ is an $R_{1}$-algebra, and suppose that $\mathscr{E}$ is a quasicoherent sheaf over $\mathrm{X}_{\mathcal{H}, \Sigma}^{\text {tor }}$ that is formally canonical over $R$, as in Definition 2.5.8. Then the canonical restriction morphism

$$
\left(\mathrm{Y}_{\Sigma}^{\text {tor }} \rightarrow \mathrm{Y}^{\mathrm{min}}\right)_{*} \mathscr{E} \rightarrow\left(\mathrm{Y} \rightarrow \mathrm{Y}^{\mathrm{min}}\right)_{*}(\mathscr{E} \mid \mathrm{Y})
$$

is an isomorphism. Consequently, for each open subset $U^{\min }$ of $\mathrm{Y}^{\mathrm{min}}$, if we denote by $U_{\Sigma}^{\text {tor }}$ its preimage in $\mathrm{Y}_{\Sigma}^{\text {tor }}$ under the canonical morphisms $\oint_{\mathrm{Y}, \Sigma}$, and by $U$ its preimage in $\mathrm{Y}$ under the canonical morphism $\mathrm{Y} \rightarrow \mathrm{Y}^{\mathrm{min}}$, then the canonical restriction map

$$
\Gamma\left(U_{\Sigma}^{\text {tor }},\left.\mathscr{E}\right|_{U_{\Sigma}^{\text {tor }}}\right) \rightarrow \Gamma\left(U,\left.\mathscr{E}\right|_{U}\right)
$$

is a bijection, except when $\operatorname{dim}\left(\mathrm{X}_{\mathcal{H}}\right)=1$ and $U^{\min }-U \neq \emptyset$.

Proof. Thanks to Theorem 2.3.2, which provides almost the same axiomatic setup in [39, Section 4], and thanks to Proposition 2.5.7, which implies the analogue of 
[39, Lemma 6.2] for the context here (under the assumption that $\mathcal{O} \otimes_{\mathbb{Z}} \mathbb{Q}$ is a simple algebra over $\mathbb{Q}$ ), the same argument as in the proof of [39, Theorem 2.3] also works here.

REMARK 2.5.14. For an example, see Example 4.2.24 below.

REMARK 2.5.15. Based on the arguments of the proofs of [39, Theorems 3.9 and 2.3] and of [41, Theorems 8.6 and 8.7], the proofs of Theorems 2.5.10 and 2.5.11 only make use of the underlying topological space of $Y_{Z}$, which coincides with $Y_{Z}^{\natural}$ as a subset of $Z_{T}$, and of the formal completion of $Y_{\Sigma}^{\text {tor }}$ along the preimage of $Y_{Z}$. Such arguments closely follow the formal local approaches in the proofs of [24, Theorem 5.4], [42, Section 8.2], and [19, Ch. V, Proposition 1.5], rather than the global cohomological approaches in the proofs of [44, Theorem 1.1], [39, Theorem 2.5], and [40, Theorems 4.5 and 4.6].

REMARK 2.5.16. Theorem 2.5.11 shows that, in cases where $\mathcal{O} \otimes_{\mathbb{Z}} \mathbb{Q}$ is simple and where $X_{\mathcal{H}} \rightarrow S$ is nonproper and of relative dimension at least two, any generalized Hasse invariants over the Ekedahl-Oort strata $\mathrm{Y}$ of $\left(\mathrm{X}_{\mathcal{H}}\right)_{s}$ (as in Section 3.5 below) automatically extend to $Y_{\Sigma}^{\text {tor }}$, as in [12, Theorem 6.2.2], and hence to the Stein factorization $\left(\mathrm{Y}_{\Sigma}^{\text {tor }}\right)^{\text {st }}:=\underline{\operatorname{Spec}}_{\mathscr{O}_{\mathrm{Ymin}}}\left(\left(\oint_{\mathrm{Y}, \Sigma}\right)_{*} \mathscr{O}_{\mathrm{Y}_{\Sigma}^{\text {tor }}}\right)$ of the proper surjective morphism $\oint_{Y, \Sigma}: Y_{\Sigma}^{\text {tor }} \rightarrow Y^{\text {min }}$, which is finite over $Y^{\text {min }}$. Although this does not imply that they descend to $Y^{\text {min }}$ in general, they do descend to $Y^{\text {min }}$ (with affine nonvanishing loci as usual) in the context of [12] (which is in Case (Sm) here), as in [12, Theorem 6.2.3]. This is because, in Case (Sm), by [12, Lemma 3.4.3] and its proof, the formation of $\left(\oint_{\mathcal{H}, \Sigma}\right)_{*} \mathscr{O}_{\mathbf{X}_{\mathcal{H}, \Sigma}^{\text {tor }}}$ commutes with base change to $\mathrm{Y}^{\text {min }}$ (and gives $\left(\oint_{\mathrm{Y}, \Sigma}\right)_{*} \mathscr{O}_{Y_{\Sigma}^{\text {tor }}}$ ) whenever $\mathrm{Y}$ is a well-positioned subscheme of $\left(\mathrm{X}_{\mathcal{H}}\right)_{\mathrm{T}}$; and therefore $\left(\mathrm{Y}_{\Sigma}^{\text {tor }}\right)^{\mathrm{st}} \rightarrow \mathrm{Y}^{\mathrm{min}}$ is an isomorphism in this case.

REMARK 2.5.17 (Cf. [41, Theorem 8.10]). Since the proof of [39, Theorem 2.5] made use of Serre duality, we cannot easily generalize the higher Koecher's principle to the context of Theorem 2.5.11. (We already had no idea whether we should expect such a generalization over the whole integral models in ramified characteristics.)

\section{Examples of well-positioned subsets and subschemes}

3.1. Pullbacks and fibers. Let $X_{\mathcal{H}} \rightarrow S$ be as in Assumption 2.1.1. For any locally noetherian scheme $T$ over $S$, it is tautological that the whole scheme $Y=$ $\left(X_{\mathcal{H}}\right)_{\mathrm{T}}$ is a well-positioned subscheme of itself, that $\mathrm{Y}^{\natural}=\left\{Z_{\mathrm{T}}\right\}_{\mathrm{Z}}$ is associated 
with $Y$ as in Definition 2.2.1, and so that $Y^{\min }=\left(X_{\mathcal{H}}^{\min }\right)_{\mathrm{T}}$ and $Y_{\Sigma}^{\text {tor }}=\left(X_{\mathcal{H}, \Sigma}^{\text {tor }}\right)_{\mathrm{T}}$ satisfy the properties in Theorem 2.3.2, analogous to those in Proposition 2.1.2. In particular, the fibers and geometric fibers of $X_{\mathcal{H}}, X_{\mathcal{H}}^{\min }$, and $X_{\mathcal{H}, \Sigma}^{\text {tor }}$ over $S$ admit the same stratifications and the formal local descriptions as in Proposition 2.1.2.

This is not as trivial as it seems to be. In all cases in Assumption 2.1.1, even the fiberwise density of $X_{\mathcal{H}}$ in $X_{\mathcal{H}}^{\min }$ is not obvious and required some hard work, let alone the stratification and formal local descriptions along the boundary. (Nevertheless, these are all proved in the works [36, 38, 41, 43, 50] we cited in the proof of Proposition 2.1.2 or rather [45, Proposition 2.2].)

3.2. $\quad$-rank strata and their pullbacks. We shall consider only Cases ( $\mathrm{Sm})$, $(\mathrm{Nm})$, or $(\mathrm{Spl})$ in this subsection. (As explained in the introduction, we have chosen to present our examples here and in later subsections only in PEL-type cases, because the theories are most complete and well understood in these cases. We have not tried to include Case (Hdg) because the corresponding theories are still developing.) Let $\mathrm{X}_{\mathcal{H}} \rightarrow \mathrm{S}$ be as in Assumption 2.1.1, which carries a tautological collection $\left\{\left(A_{\mathrm{j}}, \lambda_{\mathrm{j}}, i_{\mathrm{j}}\right)\right\}_{\mathrm{j} \in \mathrm{J}}$ of abelian varieties quasi-isogenous to each other over $\mathrm{X}_{\mathcal{H}}$, equipped with polarizations and endomorphism structures. (In Case ( $\mathrm{Sm})$, the index set $\mathrm{J}$ is just a singleton.) Let $\mathrm{T} \rightarrow \mathrm{S}$ be the special point $s=\operatorname{Spec}(k) \rightarrow \mathrm{S}$ of residue characteristic $p>0$.

Consider any geometric point $\bar{t} \rightarrow\left(\mathrm{X}_{\mathcal{H}}\right)_{s}$ above a point $t \in\left(\mathrm{X}_{\mathcal{H}}\right)_{s}$, which defines by pullback a collection $\left\{\left(A_{\mathrm{j}, \bar{t}}, \lambda_{\mathrm{j}, \bar{t}}, i_{\mathrm{j}, \bar{t}}\right)\right\}_{\mathrm{j} \in \mathrm{J}}$ of abelian varieties quasiisogenous to each other over $\bar{t}$, equipped with polarizations and endomorphism structures. Since the $p$-rank of an abelian variety is an isogeny invariant (see [63, Ch. III, Section 15, page 147]), the $p$-rank of $\bar{t} \rightarrow\left(\mathrm{X}_{\mathcal{H}}\right)_{s}$, which we shall denote as $r(\bar{t})$, can be defined to be the $p$-rank of $A_{\mathrm{j}, \bar{t}}$ for any $\mathrm{j} \in \mathrm{J}$. Since the $p$-rank of $A_{\mathrm{j}, \bar{t}}$ is unchanged under any automorphism of $\bar{t} \rightarrow\left(\mathrm{X}_{\mathcal{H}}\right)_{s}$, it is unambiguous to write $r(t):=r(\bar{t})$.

Since the $p$-rank of an $m$-fold self-fiber product of an abelian variety $A$ is just $m$ times the $p$-rank of $A$, by considering any morphism from $\left(\mathrm{X}_{\mathcal{H}}\right)_{s}$ to some principally polarized Siegel moduli with no level at $p$ (using Zarhin's trick if necessary, as in the constructions in [38, Lemma 4.1(2) and (4.6)]), and by pulling back the $p$-rank strata over (some characteristic $p$ fiber of) such Siegel moduli (cf. [30, Section IV.1]), we obtain the following:

PROPOSITION 3.2.1.

(1) The subset

$$
\left(\mathrm{X}_{\mathcal{H}}\right)_{s}^{(r)}:=\left\{t \in\left(\mathrm{X}_{\mathcal{H}}\right)_{s}: r(t)=r\right\}
$$

of $\left(\mathrm{X}_{\mathcal{H}}\right)_{s}$ is locally closed, and hence admits the structure of a reduced subscheme, for each integer $r \geqslant 0$. 
(2) The union $\bigcup_{0 \leqslant r \leqslant r_{0}}\left(\mathrm{X}_{\mathcal{H}}\right)_{s}^{(r)}$ is closed for each integer $r_{0} \geqslant 0$.

(3) We have a set-theoretic disjoint union

$$
\left(\mathbf{X}_{\mathcal{H}}\right)_{s}=\coprod_{r \geqslant 0}\left(\mathbf{X}_{\mathcal{H}}\right)_{s}^{(r)} .
$$

REMARK 3.2.3. The disjoint union (3.2.2) is not a stratification in general, because the closure of $\left(\mathbf{X}_{\mathcal{H}}\right)_{s}^{\left(r_{0}\right)}$ in $\left(\mathbf{X}_{\mathcal{H}}\right)_{s}$ might be smaller than $\bigcup_{0 \leqslant r \leqslant r_{0}}\left(\mathbf{X}_{\mathcal{H}}\right)_{s}^{(r)}$ for some $r_{0}$. (See, for example, [22] for the case of Siegel moduli with Iwahori levels at $p$.)

DEFinition 3.2.4. By abuse of language, we shall still call $\left(\mathrm{X}_{\mathcal{H}}\right)_{s}^{(r)}$ the $p$-rank $r$ stratum of $\left(\mathrm{X}_{\mathcal{H}}\right)_{s}$. We shall call any such stratum a p-rank stratum.

Proposition 3.2.5. For each $r_{0} \geqslant 0$, the locally closed subset $\mathrm{Y}:=\left(\mathrm{X}_{\mathcal{H}}\right)_{s}^{\left(r_{0}\right)}$ of $\left(\mathrm{X}_{\mathcal{H}}\right)_{s}$ is a well-positioned subset as in Definition 2.2.1, which is associated with some collection $Y^{\natural}=\left\{Y_{Z}^{\natural}\right\}_{Z}$ such that $Y_{Z}^{\natural}$ is either the empty subset, or some $p$ rank stratum of $Z_{s}$ (whose precise definition will be made clear in the proof), for each $\mathrm{Z}$. With its reduced subscheme structure, $\left(\mathrm{X}_{\mathcal{H}}\right)_{s}^{\left(r_{0}\right)}$ admits the partial minimal and toroidal compactifications $\left(\mathrm{X}_{\mathcal{H}}^{\min }\right)_{s}^{\left(r_{0}\right)}:=\mathrm{Y}^{\min }$ and $\left(\mathrm{X}_{\mathcal{H}, \Sigma}^{\text {tor }}\right)_{s}^{\left(r_{0}\right)}:=\mathrm{Y}_{\Sigma}^{\text {tor }}$ as in Definition 2.3.1 and Theorem 2.3.2. By Lemma 2.2.10, when $C \rightarrow Z$ is reduced (which is the case, for example, when $C \rightarrow \mathrm{Z}$ is smooth), $\left(\mathrm{X}_{\mathcal{H}}\right)_{s}^{\left(r_{0}\right)}$ (with its reduced subscheme structure) is also a well-positioned subscheme. These statements are also true if we consider the closed union $\bigcup_{0 \leqslant r \leqslant r_{0}}\left(\mathbf{X}_{\mathcal{H}}\right)_{s}^{(r)}$ instead of $\left(\mathrm{X}_{\mathcal{H}}\right)_{s}^{\left(r_{0}\right)}$.

To show this, we need the following (reviewing) lemma, which will also be useful for the consideration of more complicated strata in later sections:

Lemma 3.2.6. For each $W$ as in Proposition 2.1.3, let $\left\{\left(A_{\mathrm{j}, W^{0}}, \lambda_{\mathrm{j}, W^{0}}, i_{\mathrm{j}, W^{0}}\right)\right\}_{\mathrm{j} \in \mathrm{J}}$ denote the pullback of $\left\{\left(A_{\mathrm{j}}, \lambda_{\mathrm{j}}, i_{\mathrm{j}}\right)\right\}_{\mathrm{j} \in \mathrm{J}}$ to $W^{0}$. By considering also the pullbacks of the Mumford families over $\mathfrak{X}_{\sigma}^{\circ}$ (see Proposition 2.1.3), the compatible collection of polarizations $\left\{\lambda_{\mathrm{j}, W^{0}}: A_{\mathrm{j}, W^{0}} \rightarrow A_{\mathrm{j}, W^{0}}^{\vee}\right\}_{\mathrm{j} \in \mathrm{J}}$ of abelian schemes (which are compatible with their endomorphism structures) extends to a compatible collection of homomorphisms $\left\{\lambda_{\mathrm{j}, W}: G_{\mathrm{j}, W} \rightarrow G_{\mathrm{j}, W}^{\vee}\right\}_{\mathrm{j} \in \mathrm{J}}$ between semi-abelian schemes with canonically extended endomorphism structures. Since $W$ is noetherian and normal (by [21, IV-2, 7.8.3(v)]), the above determines a compatible collection of homomorphisms $\left\{\lambda_{\mathrm{j}, W}^{\natural}: G_{\mathrm{j}, W}^{\natural} \rightarrow G_{\mathrm{j}, W}^{\vee, \natural}\right\}_{\mathrm{j} \in \mathrm{J}}$ between their Raynaud extensions (see [36, Sections 3.3.3 and 3.4.4]), together with a compatible collection of commutative diagrams 


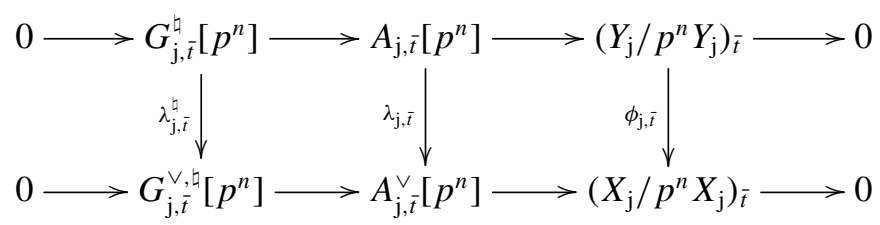

and

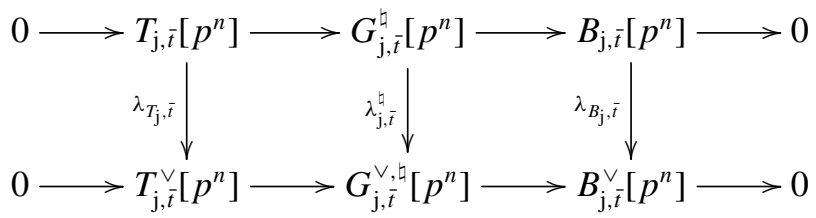

of finite flat group schemes over each geometric point $\bar{t} \rightarrow W^{0}$, for each integer $n \geqslant 1$, where $T_{\mathrm{j}, W}$ (respectively $B_{\mathrm{j}, W}$ ) is the torus (respectively abelian) part of $G_{\mathrm{j}, W}^{\natural}$, where $T_{\mathrm{j}, W}^{\vee}$ (respectively $B_{\mathrm{j}, W}^{\vee}$ ) is the torus (respectively abelian) part of $G_{\mathrm{j}, W}^{\vee}$, where $X_{\mathrm{j}}$ and $Y_{\mathrm{j}}$ are the respective character groups of $T_{\mathrm{j}}$ and $T_{\mathrm{j}}^{\vee}$, where the objects with subscripts $\bar{t}$ are pullbacks to $\bar{t}$ of the corresponding objects over $W^{0}$ or $W$, where the vertical morphisms are all induced by polarizations, and where the horizontal morphisms are all exact sequences of finite flat group schemes.

Proof. These assertions follow from the corresponding assertions for Mumford families. (See [36, Chs 4 and 5, and Section 6.2.5] for Mumford's construction and for the definition of Mumford families, and see more particularly [36, Corollary 4.5.2.13 and Proposition 5.2.2.1] for the assertions concerning torsion points.)

Proof of Proposition 3.2.5. By Lemma 3.2.6, for each $\mathrm{j} \in \mathrm{J}$ and for each geometric point $\bar{t} \rightarrow W^{0}$, since $\left(Y / p^{n} Y\right)_{\bar{t}}$ is constant (étale) and since $T_{\mathrm{j}, \bar{t}}$ is a torus (and hence $T_{\mathrm{j}, \bar{t}}\left[p^{n}\right]$ is of multiplicative type for every $n \geqslant 1$ ), the $p$-rank of $A_{\mathrm{j}, \bar{t}}$ is just $\mathrm{rk}_{\mathbb{Z}}(Y)$ plus the $p$-rank of the abelian part $B_{\mathrm{j}, \bar{t}}$, which depends only on the composition $\bar{t} \rightarrow W^{0} \rightarrow \mathrm{Z}$, under which $\left(B_{\mathrm{j}, \bar{t}}, \lambda_{B_{\mathrm{j}, \bar{t}}}, i_{B_{\mathrm{j}, \bar{t}}}\right)$ is the pullback of the tautological $\left(B_{\mathrm{j}}, \lambda_{B_{\mathrm{j}}}, i_{B_{\mathrm{j}}}\right)$ over $\mathrm{Z}$. Hence, $\mathrm{Y}:=\left(\mathrm{X}_{\mathcal{H}}\right)_{s}^{\left(r_{0}\right)}$ is a well-positioned subset as in Definition 2.2.1 if, when $r_{0} \geqslant \operatorname{rk}_{\mathbb{Z}}(Y)$, we take $Y_{Z}^{\natural}:=Z_{s}^{r_{0}-\mathrm{rk}_{\mathbb{Z}}(Y)}$, the $p$-rank $r_{0}-\operatorname{rk}_{\mathbb{Z}}(Y)$ stratum of $Z_{s}$ (defined similarly by the tautological collection $\left\{\left(B_{\mathrm{j}}, \lambda_{B_{\mathrm{j}}}, i_{B_{\mathrm{j}}}\right)\right\}_{\mathrm{j} \in \mathrm{J}}$ over $\left.Z\right)$; and when $r_{0}<\operatorname{rk}_{\mathbb{Z}}(Y)$, we take $Y_{Z}^{\natural}:=\emptyset$. The remaining assertions in the proposition are self-explanatory.

REMARK 3.2.9. When $C \rightarrow \mathrm{Z}$ is reduced at some level $\mathcal{H}$, so that $\left(\mathrm{X}_{\mathcal{H}}\right)_{s}^{(r)}$ is a well-positioned subscheme of $\left(\mathrm{X}_{\mathcal{H}}\right)_{s}$ by Proposition 3.2.5, for each integer $r \geqslant 0$, 
the pullback of $\left(\mathrm{X}_{\mathcal{H}}\right)_{s}^{(r)}$ to $\left(\mathrm{X}_{\mathcal{H}^{\prime}}\right)_{s}$ for any higher level $\mathcal{H}^{\prime} \subset \mathcal{H}$ is a well-positioned subscheme of $\left(\mathrm{X}_{\mathcal{H}^{\prime}}\right)_{s}$, by Proposition 2.4.2, which underlies the same subset as $\left(\mathrm{X}_{\mathcal{H}^{\prime}}\right)_{s}^{(r)}$. This is useful, for example, when $p$ is a good prime for $(\mathcal{O}, \star, L,\langle\cdot, \cdot\rangle$, $\left.h_{0}\right)$ as in [36, Definition 1.4.1.1], in which case there exists a bottom level $\mathcal{H}$ at which the morphisms $C \rightarrow \mathrm{Z}$ are all smooth. Then we can pullback from such a bottom level and obtain well-positioned subschemes over the $p$-rank strata in all higher levels.

3.3. Newton strata and their pullbacks. We shall consider only Cases ( $\mathrm{Sm})$, $(\mathrm{Nm})$, or $(\mathrm{Spl})$ in this subsection. Let $\mathrm{X}_{\mathcal{H}} \rightarrow \mathrm{S}$ be as in Assumption 2.1.1, which carries a tautological collection $\left\{\left(A_{\mathrm{j}}, \lambda_{\mathrm{j}}, i_{\mathrm{j}}, \alpha_{\mathcal{H}_{\mathrm{j}}}\right)\right\}_{\mathrm{j} \in \mathrm{J}}$, as in the beginning of Section 3.2. Let us take $\mathrm{T} \rightarrow \mathrm{S}$ to be the special point $s=\operatorname{Spec}(k) \rightarrow \mathrm{S}$. For simplicity, assume that $\mathcal{O} \otimes_{\mathbb{Z}} \mathbb{Q}$ involves no factor of type $\mathrm{D}$, in the sense of [36, Definition 1.2.1.15], so that (any pullback of) $G \otimes_{\mathbb{Z}} \mathbb{Q}$ is (fiberwise) connected.

Consider any geometric point $\bar{t}=\operatorname{Spec}(k(\bar{t})) \rightarrow\left(\mathrm{X}_{\mathcal{H}}\right)_{s}$ above a point $t \in\left(\mathrm{X}_{\mathcal{H}}\right)_{s}$, which defines by pullback a collection $\left\{\left(A_{\mathrm{j}, \bar{t}}, \lambda_{\mathrm{j}, \bar{t}}, i_{\mathrm{j}, \bar{t}}\right)\right\}_{\mathrm{j} \in \mathrm{J}}$ as before, and hence also a collection

$$
\left\{\left(A_{\mathrm{j}, \bar{t}}\left[p^{\infty}\right], \lambda_{\mathrm{j}, \bar{t}}, i_{\mathrm{j}, \bar{t}}\right)\right\}_{\mathrm{j} \in \mathrm{J}}
$$

of Barsotti-Tate groups with quasipolarizations and endomorphism structures. Consider

$$
K_{\bar{t}}:=\operatorname{Frac}(W(k(\bar{t}))),
$$

which is equipped with the Frobenius automorphism $\sigma_{\bar{t}}$ induced by the $p$ th power automorphism of $k(\bar{t})$. Following [31] and [74, Section 1], we say that two elements $x, y \in \mathrm{G}\left(K_{\bar{t}}\right)$ are $\sigma_{\bar{t}}$-conjugate if there exists $g \in \mathrm{G}\left(K_{\bar{t}}\right)$ such that $g^{-1} x \sigma_{\bar{t}}(g)=y$. By [75, Lemma 1.16], any morphism $\bar{t}^{\prime} \rightarrow \bar{t}$ between spectra of algebraically closed fields in characteristic $p>0$ induces a bijection from the set of $\sigma_{\bar{t}}$-conjugacy classes in $\mathrm{G}\left(K_{\bar{t}}\right)$ to the set of $\sigma_{\bar{t}^{\prime}}$-conjugacy classes in $\mathrm{G}\left(K_{\bar{t}^{\prime}}\right)$, and hence it is unambiguous to denote either of the two sets as $\mathrm{B}\left(\mathrm{G} \otimes_{\mathbb{Z}} \mathbb{Q}_{p}\right)$.

For each $\mathrm{j} \in \mathrm{J}$, the covariant Dieudonné module $\mathbb{D}\left(A_{\mathrm{j}, \bar{t}}\left[p^{\infty}\right]\right)$ of $A_{\mathrm{j}, \bar{t}}\left[p^{\infty}\right]$ is canonically isomorphic to the $W(k(\bar{t}))$-dual of $H_{\text {crys }}^{1}\left(A_{\bar{t}} / W(k(\bar{t}))\right)$, equipped with additional structures induced by $\lambda_{\mathrm{j}, \bar{t}}$ and $i_{\mathrm{j}, \bar{t}}$, and with its ( $\sigma_{\bar{t}}$-linear) Frobenius and $\left(\sigma_{\bar{t}}^{-1}\right.$-linear) Verschiebung endomorphisms, as usual. (See [54, Ch. IV], [53], and [7].) By [75, $3.23 \mathrm{c}$ )], we have compatible symplectic isomorphisms

$$
\mathbb{D}\left(A_{\mathrm{j}, \bar{t}}\left[p^{\infty}\right]\right) \cong L_{\mathrm{j}} \otimes_{\mathbb{Z}} W(k(\bar{t}))
$$

of $\mathcal{O} \otimes_{\mathbb{Z}} W\left(k_{\bar{t}}\right)$-modules, for all $\mathrm{j} \in \mathrm{J}$, inducing compatible symplectic isomorphisms

$$
\mathbb{D}\left(A_{\mathrm{j}, \bar{t}}\left[p^{\infty}\right]\right) \otimes_{W(k(\bar{t}))} K_{\bar{t}} \cong L \otimes_{\mathbb{Z}} K_{\bar{t}}
$$


of $\mathcal{O} \otimes_{\mathbb{Z}} K_{\bar{t}}$-modules. Hence, for any $\mathrm{j} \in \mathrm{J}$, the Frobenius automorphism of $\mathbb{D}\left(A_{\mathrm{j}, \bar{t}}\left[p^{\infty}\right]\right) \bigotimes_{W(k(\bar{t}))} K_{\bar{t}}$ induces a $\sigma_{\bar{t}}$-linear automorphism of $L \otimes_{\mathbb{Z}} K_{\bar{t}}$, which is independent of the choice of $\mathrm{j} \in \mathrm{J}$ and determines a well-defined $\sigma_{\bar{t}}$-conjugacy class $b(\bar{t})$ in $\mathrm{B}\left(\mathrm{G} \otimes_{\mathbb{Z}} \mathbb{Q}_{p}\right)$.

By [74, Sections 1-3, especially Theorem 3.6] and [31, Section 3], we have the following:

PROPOSITION 3.3.1.

(1) The assignment of $b(\bar{t}) \in \mathrm{B}\left(\mathrm{G} \otimes_{\mathbb{Z}} \mathbb{Q}_{p}\right)$ to a geometric point $\bar{t} \rightarrow\left(\mathrm{X}_{\mathcal{H}}\right)_{s}$ depends only on the image $t$ of $\bar{t} \rightarrow\left(\mathrm{X}_{\mathcal{H}}\right)_{s}$, in the sense that any automorphism of $\bar{t} \rightarrow\left(\mathrm{X}_{\mathcal{H}}\right)_{s}$ induces an automorphism of $\mathrm{G}\left(K_{\bar{t}}\right)$ preserving the $\sigma_{\bar{t}}$-conjugacy class of $b(\bar{t})$. Thus, it is unambiguous to write

$$
b(t):=b(\bar{t}) .
$$

(2) There is a partial ordering $\leqslant$ on the set $\mathrm{B}\left(\mathrm{G} \otimes_{\mathbb{Z}} \mathbb{Q}_{p}\right)$ such that, for each $b \in \mathrm{B}\left(\mathrm{G} \otimes_{\mathbb{Z}} \mathbb{Q}_{p}\right)$, the subset $\left\{t \in\left(\mathrm{X}_{\mathcal{H}}\right)_{s}: b(t) \leqslant b\right\}$ of $\left(\mathrm{X}_{\mathcal{H}}\right)_{s}$ is closed, and so the subset

$$
\left(\mathrm{X}_{\mathcal{H}}\right)_{s}^{b}:=\left\{t \in\left(\mathbf{X}_{\mathcal{H}}\right)_{s}: b(t)=b\right\}
$$

of $\left(\mathrm{X}_{\mathcal{H}}\right)_{s}$ is locally closed. Hence, we have a set-theoretic disjoint union

$$
\left(\mathrm{X}_{\mathcal{H}}\right)_{s}=\coprod_{b \in \mathrm{B}\left(\mathrm{G} \otimes_{\mathbb{Z}} \mathbb{Q}_{p}\right)}\left(\mathbf{X}_{\mathcal{H}}\right)_{s}^{b} .
$$

(3) There is a canonical map from $\mathrm{B}\left(\mathrm{G} \otimes_{\mathbb{Z}} \mathbb{Q}_{p}\right)$ to the set $\mathcal{N}$ of Newton polygons $\left(\right.$ for $\left.\mathrm{GL}_{\mathbb{Q}_{p}}\left(L \otimes_{\mathbb{Z}} \mathbb{Q}_{p}\right)\right)$, denoted $b \mapsto v_{b}$, such that $b \leqslant b^{\prime}$ only if $v_{b} \leqslant v_{b^{\prime}}$ (which is the case when $v_{b}$ and $v_{b^{\prime}}$ have the same end points and $v_{b}$ lies above $\left.v_{b^{\prime}}\right)$. For each geometric point $\bar{t} \rightarrow\left(\mathrm{X}_{\mathcal{H}}\right)_{s}$, the corresponding Newton polygon $v_{b(\bar{t})}$ is the one classifying the (rational) covariant Dieudonné module $\mathbb{D}\left(A_{\mathrm{j}, \bar{t}}\left[p^{\infty}\right]\right) \otimes_{W(k(\bar{t}))} K_{\bar{t}}($ for any $\mathrm{j} \in \mathrm{J})$, ignoring the additional structures.

(4) For each $v \in \mathcal{N}$, the subset $\left\{t \in\left(\mathrm{X}_{\mathcal{H}}\right)_{s}: v_{b(t)} \leqslant v\right\}$ of $\left(\mathrm{X}_{\mathcal{H}}\right)_{s}$ is closed, and so the subset

$$
\left(\mathbf{X}_{\mathcal{H}}\right)_{s}^{v}:=\left\{t \in\left(\mathbf{X}_{\mathcal{H}}\right)_{s}: v_{b(t)}=v\right\}
$$

of $\left(\mathrm{X}_{\mathcal{H}}\right)_{s}$ is locally closed. Hence, we have a set-theoretic disjoint union

$$
\left(\mathbf{X}_{\mathcal{H}}\right)_{s}=\coprod_{\nu \in \mathcal{N}}\left(\mathbf{X}_{\mathcal{H}}\right)_{s}^{v}
$$

which is coarser than (3.3.2) in general. For each $b \in \mathrm{B}\left(\mathrm{G} \otimes_{\mathbb{Z}} \mathbb{Q}_{p}\right)$, the subset $\left(\mathbf{X}_{\mathcal{H}}\right)_{s}^{b}$ of $\left(\mathbf{X}_{\mathcal{H}}\right)_{s}^{v_{b}}$ is open and closed. 
REMARK 3.3.4. The disjoint union (3.3.2) is not a stratification in general, because the closure of $\left(\mathrm{X}_{\mathcal{H}}\right)_{s}^{b}=\left\{t \in\left(\mathrm{X}_{\mathcal{H}}\right)_{s}: b(t)=b\right\}$ in $\left(\mathrm{X}_{\mathcal{H}}\right)_{s}$ might be smaller than $\left\{t \in\left(\mathrm{X}_{\mathcal{H}}\right)_{s}: b(t) \leqslant b\right\}$. (See [77] and [25, Corollary 3.11.2 and Section 3.12] for examples where the ordinary loci are nonempty but not dense.) Nevertheless, the situation is better in Case ( $\mathrm{Sm})$, where the level at $p$ is hyperspecial: By [23, Theorem 1.1], (3.3.2) is indeed a stratification. Moreover, by [83, Theorem 11.1], $\left(\mathrm{X}_{\mathcal{H}}\right)_{s}^{b}$ is nonempty for each $b \in \mathrm{B}\left(\mathrm{G} \otimes_{\mathbb{Z}} \mathbb{Q},[\mu]\right) \subset \mathrm{B}\left(\mathrm{G} \otimes_{\mathbb{Z}} \mathbb{Q}\right)$, where $[\mu]$ is the conjugacy class of cocharacters determined by $h_{0}$ as in [45, Section 6.1], and where $\mathrm{B}\left(\mathrm{G} \otimes_{\mathbb{Z}} \mathbb{Q},[\mu]\right)$ is as in $[33$, Section 6].

Definition 3.3.5. By abuse of language, we shall still call each $\left(\mathrm{X}_{\mathcal{H}}\right)_{s}^{b}$ a Newton stratum of $\left(\mathrm{X}_{\mathcal{H}}\right)_{s}$.

Let $Z$ be a stratum of $X_{\mathcal{H}}^{\min }$. (At least temporarily, we need to introduce some filtrations $Z$ and $V$, where $Z$ is typeset in a very slightly different font compared with $Z$. This could be a bit confusing, but we hope the purpose of the notation will be clear from the context.) Since we are in Cases $(\mathrm{Sm}),(\mathrm{Nm})$, or $(\mathrm{Spl})$, the stratum $\mathrm{Z}$ is associated with some cusp label $\left[\left(\mathrm{Z}_{\mathcal{H}}, \Phi_{\mathcal{H}}, \delta_{\mathcal{H}}\right)\right]$, which determines an $\mathcal{H}$-orbit $Z_{\mathcal{H}}$ of a fully symplectic liftable filtration $Z=\left\{Z_{-i}\right\}_{i \in \mathbb{Z}}$ on $L \otimes_{\mathbb{Z}} \hat{\mathbb{Z}}$ (see [36, Definitions 5.2.7.1 and 5.4.2.4]). By [37, Proposition A.5.8 and Lemma A.4.3], we have the following:

Lemma 3.3.6. In Cases (Sm), (Nm), or (Spl), under the assumption that $\mathrm{G} \otimes_{\mathbb{Z}} \mathbb{Q}$ is connected, there exists a parabolic subgroup $\mathrm{P}$ of $\mathrm{G} \otimes_{\mathbb{Z}} \mathbb{Q}$, which is the stabilizer of a symplectic filtration $\mathrm{V}=\left\{\mathrm{V}_{-i}\right\}_{i \in \mathbb{Z}}$ of $L \otimes_{\mathbb{Z}} \mathbb{Q}$, with

$$
0=\mathrm{V}_{-3} \subset \mathrm{V}_{-2} \subset \mathrm{V}_{-2}^{\perp}=\mathrm{V}_{-1} \subset \mathrm{V}_{0}=L \otimes_{\mathbb{Z}} \mathbb{Q},
$$

such that $\mathrm{V}_{-2} \otimes_{\mathbb{Q}} \mathbb{A}^{\infty}$ lies in the $\mathcal{H}$-orbit of $\mathrm{Z}_{-2} \otimes_{\mathbb{Z}} \mathbb{Q}$ for some representative $\mathrm{Z}$ of $\mathrm{Z}_{\mathcal{H}}$. The image of $\mathrm{P}$ in each simple factor of $\left(\mathrm{G} \otimes_{\mathbb{Z}} \mathbb{Q}\right)^{\text {ad }}$ is either the whole factor or a proper maximal parabolic subgroup.

REMARK 3.3.7. Lemma 3.3.6 is generally false without the assumption that $\mathrm{G} \otimes_{\mathbb{Z}}$ $\mathbb{Q}$ is connected. See [37, Example A.7.2].

Let us fix the choices of $\mathrm{P}$ and $\mathrm{V}$ as in Lemma 3.3.6.

Definition 3.3.8. For each $i$, set $\mathrm{Gr}_{-i}^{\mathrm{V}}=\mathrm{V}_{-i} / \mathrm{V}_{-i-1}$ as usual. Then $\mathrm{Gr}_{-1}^{\mathrm{V}}$ is equipped with a pairing $\langle\cdot, \cdot\rangle_{-1}$ induced by $\langle\cdot, \cdot\rangle$, compatible with $\mathcal{O}$-actions in the sense that $\langle b x, y\rangle_{-1}=\left\langle x, b^{\star} y\right\rangle_{-1}$ for all $b \in \mathcal{O}$ and $x, y \in \mathrm{Gr}_{-1}^{\mathrm{V}}$. 
Consider, for each $\mathbb{Q}$-algebra $R$, the following quotients of subgroups of $\mathrm{P}(R)$ :

(1) $\mathrm{P}^{\prime}(R)$ is the kernel of the homomorphism

$$
\begin{aligned}
\left(v^{-1} \mathrm{Gr}_{-2}^{\mathrm{V}}\right)(R) \times \mathrm{Gr}_{0}^{\mathrm{V}}(R): \mathrm{P}(R) \rightarrow & \mathrm{GL}_{\mathcal{O} \otimes_{\mathbb{Z}} R}\left(\mathrm{Gr}_{-2}^{\mathrm{V}} \otimes_{\mathbb{Q}} R\right) \\
& \times \mathrm{GL}_{\mathcal{O} \otimes_{\mathbb{Z} R}}\left(\mathrm{Gr}_{0}^{\mathrm{V}} \otimes_{\mathbb{Q}} R\right): \\
(g, r) \mapsto & \left(r^{-1} \mathrm{Gr}_{-2}^{\mathrm{V}}(g), \mathrm{Gr}_{0}^{\mathrm{V}}(g)\right),
\end{aligned}
$$

where $v(R): \mathrm{P}(R) \rightarrow \mathbf{G}_{\mathrm{m}}(R):(g, r) \mapsto r$ denotes the similitude character.

(2) $\mathrm{U}(R):=\left\{g \in \mathrm{P}: \operatorname{Gr}^{\mathrm{V}}(g)=\operatorname{Id}_{\mathrm{Gr}^{\mathrm{v}}}\right\}$.

(3) $\mathrm{M}(R):=\mathrm{P}(R) / \mathrm{U}(R)$.

(4) $\mathrm{G}_{h}(R):=\left\{\begin{array}{c}\left(g_{h}, r\right) \in \mathrm{GL}_{\mathcal{O} \otimes_{\mathbb{Z}} R}\left(\mathrm{Gr}_{-1}^{\mathrm{V}} \otimes_{\mathbb{Q}} R\right) \times R^{\times}: \\ \left\langle g_{h} x, g_{h} y\right\rangle_{-1}=r\langle x, y\rangle_{-1}, \forall x, y \in \mathrm{V}_{-1}\end{array}\right\}$, which is equipped with a canonical homomorphism

$$
\mathrm{Gr}_{-1}^{\mathrm{V}}(R): \mathrm{P}(R) \rightarrow \mathrm{G}_{h}(R):(g, r) \mapsto\left(g_{h}:=\mathrm{Gr}_{-1}^{\mathrm{V}}(g), r\right) .
$$

Also, the canonical homomorphism $\mathrm{P}^{\prime}(R) \rightarrow \mathrm{G}_{h}(R)$ induces a canonical isomorphism $\mathrm{P}^{\prime}(R) / \mathrm{U}(R) \stackrel{\sim}{\rightarrow} \mathrm{G}_{h}(R)$.

(5) $\mathrm{Z}(R):=\operatorname{ker}\left(\mathrm{Gr}_{-1}^{\mathrm{V}}(R)\right)$, which contains $\mathrm{U}(R)$ by definition.

(6) $\mathrm{G}_{l}(R):=\mathrm{Z}(R) / \mathrm{U}(R) \cong \mathrm{M}(R) / \mathrm{G}_{h}(R)$, and so $\mathrm{M}(R) \cong \mathrm{G}_{l}(R) \times \mathrm{G}_{h}(R)$.

These assignments are functorial in $R$, and define the unipotent radical $\mathrm{U}$ of $\mathrm{P}$, the Levi quotient $\mathrm{M} \cong \mathrm{P} / \mathrm{U}$, and a canonical factorization $\mathrm{M} \cong \mathrm{G}_{l} \times \mathrm{G}_{h}$. For each $\mathcal{H} \subset \mathrm{G}\left(\mathbb{A}^{\infty}\right)$, we define $\mathcal{H}_{\mathrm{P}}:=\mathcal{H} \cap \mathrm{P}\left(\mathbb{A}^{\infty}\right), \mathcal{H}_{\mathrm{P}}^{\prime}:=\mathcal{H} \cap \mathrm{P}^{\prime}\left(\mathbb{A}^{\infty}\right), \mathcal{H}_{\mathrm{U}}:=\mathcal{H} \cap \mathrm{U}\left(\mathbb{A}^{\infty}\right)$, $\mathcal{H}_{\mathrm{M}}:=\mathcal{H}_{\mathrm{P}} / \mathcal{H}_{\mathrm{U}}, \mathcal{H}_{l}:=\mathcal{H}_{\mathrm{M}} \cap \mathrm{G}_{l}\left(\mathbb{A}^{\infty}\right), \mathcal{H}_{l}^{\prime}:=\mathcal{H}_{\mathrm{P}} / \mathcal{H}_{\mathrm{P}}^{\prime}, \mathcal{H}_{h}:=\mathcal{H}_{\mathrm{M}} / \mathcal{H}_{l}$, and $\mathcal{H}_{h}^{\prime}:=$ $\mathcal{H}_{\mathrm{P}}^{\prime} / \mathcal{H}_{\mathrm{U}}$. Moreover, for each torus argument $\Phi$ representing $\Phi_{\mathcal{H}}$, we define $\mathcal{H}_{h, \Phi}$ to be the image in $\mathrm{G}_{h}\left(\mathbb{A}^{\infty}\right)$ of the stabilizer of $\Phi$ in $\mathcal{H}_{\mathrm{P}}$, so that $\mathcal{H}_{h}^{\prime} \subset \mathcal{H}_{h, \Phi} \subset \mathcal{H}_{h}$.

Proposition 3.3.9. For each $b \in \mathrm{B}\left(\mathrm{G} \otimes_{\mathbb{Z}} \mathbb{Q}_{p}\right)$, the (possibly empty) locally closed subset $\mathrm{Y}:=\left(\mathrm{X}_{\mathcal{H}}\right)_{s}^{b}$ of $\left(\mathrm{X}_{\mathcal{H}}\right)_{s}$ is a well-positioned subset as in Definition 2.2.1, which is associated with some collection $Y^{\natural}=\left\{Y_{Z}^{\natural}\right\}_{Z}$ such that $\mathrm{Y}_{\mathrm{Z}}^{\natural}$ is either empty or some Newton stratum of $\mathrm{Z}_{s}$ (whose precise definition will be made clear in the proof), for each $\mathrm{Z}$. With its reduced subscheme structure, $\left(\mathrm{X}_{\mathcal{H}}\right)_{s}^{b}$ admits the partial minimal and toroidal compactifications $\left(\mathbf{X}_{\mathcal{H}}^{\min }\right)_{s}^{b}:=\mathrm{Y}^{\min }$ and $\left(\mathrm{X}_{\mathcal{H}, \Sigma}^{\text {tor }}\right)_{s}^{b}:=\mathrm{Y}_{\Sigma}^{\text {tor }}$ as in Definition 2.3.1 and Theorem 2.3.2. By Lemma 2.2.10, when $C \rightarrow \mathrm{Z}$ is reduced (which is the case, for example, when $C \rightarrow \mathrm{Z}$ is smooth), $\left(\mathrm{X}_{\mathcal{H}}\right)_{s}^{b}$ (with its reduced subscheme structure) is also a well-positioned 
subscheme. The analogous statements are true if we consider the closed union $\bigcup_{b^{\prime} \leqslant b}\left(\mathrm{X}_{\mathcal{H}}\right)_{s}^{b^{\prime}}=\left\{t \in\left(\mathrm{X}_{\mathcal{H}}\right)_{s}: b(t) \leqslant b\right\}$ instead of $\left(\mathrm{X}_{\mathcal{H}}\right)_{s}^{b}$. By Lemma 2.2 .5 and by (4) of Proposition 3.3.1, the analogous statements are also true if we consider $\left(\mathrm{X}_{\mathcal{H}}\right)_{s}^{v}$ and the closed union $\bigcup_{v^{\prime} \leqslant v}\left(\mathbf{X}_{\mathcal{H}}\right)_{s}^{v^{\prime}}$, for each $v \in \mathcal{N}$.

Proof. By Lemma 3.2.6, for each $\mathrm{j} \in \mathrm{J}$, for each $W$ as in Proposition 2.1.3, and for each geometric point $\bar{t} \rightarrow W^{0}$, the Barsotti-Tate group $A_{\mathrm{j}, \bar{t}}\left[p^{\infty}\right]$ admits a filtration with filtered pieces $0 \subset T_{\mathrm{j}, \bar{t}}\left[p^{\infty}\right] \subset G_{\mathrm{j}, \bar{t}}^{\natural}\left[p^{\infty}\right] \subset A_{\mathrm{j}, \bar{t}}\left[p^{\infty}\right]$, with the graded pieces given by the (multiplicative-type) torus part $T_{\mathrm{j}, \bar{t}}\left[p^{\infty}\right]$, the abelian part $B_{\mathrm{j}, \bar{t}}\left[p^{\infty}\right]$, and the (étale) constant part $\left(Y_{\mathrm{j}} \otimes_{\mathbb{Z}}\left(\mathbb{Q}_{p} / \mathbb{Z}_{p}\right)\right)_{\bar{t}}$, compatibly equipped with quasipolarizations and endomorphism structures. Hence, by functoriality, the associated covariant Dieudonné module $\mathbb{D}\left(A_{\mathrm{j}, \bar{t}}\left[p^{\infty}\right]\right)$ carries a symplectic filtration

$$
0 \subset \mathbb{D}\left(T_{\mathrm{j}, \bar{t}}\left[p^{\infty}\right]\right) \subset \mathbb{D}\left(G_{\mathrm{j}, \bar{t}}^{\natural}\left[p^{\infty}\right]\right) \subset \mathbb{D}\left(A_{\mathrm{j}, \bar{t}}\left[p^{\infty}\right]\right)
$$

by $\mathcal{O} \otimes_{\mathbb{Z}} W(k(\bar{t}))$-submodules.

The bottom totally isotropic piece $\mathbb{D}\left(T_{\mathrm{j}, \bar{t}}\left[p^{\infty}\right]\right)$ of (3.3.10) has the same $\mathcal{O}$-multirank (see [36, Definition 1.2.1.25]) as $Z_{-2}$, where $Z$ is any representative of the $\mathcal{H}$-orbit $Z_{\mathcal{H}}$ underlying the cusp label of $Z$ (see [36, Definitions 5.4.2.4 and 5.4.2.7, and Theorem 7.2.4.1(4)]). By [37, Lemmas A.4.3 and A.4.4] and their proofs, up to modifying the choices of the above symplectic isomorphisms $\mathbb{D}\left(A_{\mathrm{j}, \bar{t}}\left[p^{\infty}\right]\right) \otimes_{W(k(\bar{t}))} K_{\bar{t}} \cong L \otimes_{\mathbb{Z}} K_{\bar{t}}$ of $\mathcal{O} \otimes_{\mathbb{Z}} K_{\bar{t}}$-modules, which still define the same element $b(\bar{t}) \in \mathrm{B}\left(\mathrm{G} \otimes_{\mathbb{Z}} \mathbb{Q}_{p}\right)$, we may assume that these isomorphisms match (3.3.10) with the filtration $\mathrm{Z} \otimes_{\hat{\mathbb{Z}}} K_{\bar{t}}$ on $L \otimes_{\mathbb{Z}} K_{\bar{t}}$. By [37, Proposition A.5.8], there exists a totally isotropic $\mathcal{O} \otimes_{\mathbb{Z}} \mathbb{Q}$-submodule $V_{-2}$ of $L \otimes_{\mathbb{Z}} \mathbb{Q}$ such that $V_{-2} \otimes_{\mathbb{Q}} \mathbb{A}^{\infty}$ lies in the $\mathcal{H}$-orbit of $Z_{-2} \otimes_{\mathbb{Z}} \mathbb{Q}$, whose stabilizer defines a parabolic subgroup $\mathrm{P}$ of $\mathrm{G} \otimes_{\mathbb{Z}} \mathbb{Q}$, as in Lemma 3.3.6. Let $\mathrm{M}, \mathrm{G}_{l}$, and $\mathrm{G}_{h}$ be defined as in Definition 3.3.8. Let $\mathrm{B}\left(\mathrm{P} \otimes_{\mathbb{Q}} \mathbb{Q}_{p}\right)$ denote the sets of $\sigma_{\bar{t}}$-conjugacy classes in $\mathrm{P}\left(K_{\bar{t}}\right)$, and let us similarly define $\mathrm{B}\left(\mathrm{M} \otimes_{\mathbb{Q}} \mathbb{Q}_{p}\right), \mathrm{B}\left(\mathrm{G}_{l} \otimes_{\mathbb{Q}} \mathbb{Q}_{p}\right)$, and $\mathrm{B}\left(\mathrm{G}_{h} \otimes_{\mathbb{Q}} \mathbb{Q}_{p}\right)$. Then we have canonical maps

$$
\mathrm{B}\left(\mathrm{P} \otimes_{\mathbb{Q}} \mathbb{Q}_{p}\right) \rightarrow \mathrm{B}\left(\mathrm{G} \otimes_{\mathbb{Z}} \mathbb{Q}_{p}\right)
$$

and

$$
\mathrm{B}\left(\mathrm{P} \otimes_{\mathbb{Q}} \mathbb{Q}_{p}\right) \rightarrow \mathrm{B}\left(\mathrm{M} \otimes_{\mathbb{Q}} \mathbb{Q}_{p}\right) \cong \mathrm{B}\left(\mathrm{G}_{l} \otimes_{\mathbb{Q}} \mathbb{Q}_{p}\right) \times \mathrm{B}\left(\mathrm{G}_{h} \otimes_{\mathbb{Q}} \mathbb{Q}_{p}\right)
$$

induced by the canonical homomorphisms between the groups. By repeating the definition of $b(\bar{t})$, the isomorphisms $\mathbb{D}\left(A_{\mathrm{j}, \bar{t}}\left[p^{\infty}\right]\right) \otimes_{W(k(\bar{t}))} K_{\bar{t}} \cong L \otimes_{\mathbb{Z}} K_{\bar{t}}$ above, which we have assumed to match the filtrations on both sides, define an element $b_{\mathrm{P}}(\bar{t}) \in \mathrm{B}\left(\mathrm{P} \otimes_{\mathbb{Q}} \mathbb{Q}_{p}\right)$ whose image under $\mathrm{B}\left(\mathrm{P} \otimes_{\mathbb{Q}} \mathbb{Q}_{p}\right) \rightarrow \mathrm{B}\left(\mathrm{G} \otimes_{\mathbb{Z}} \mathbb{Q}_{p}\right)$ is $b(\bar{t})$.

By [33, Sections 1.4 and 3.6], $b(\bar{t})$ is determined by the image $b_{\mathrm{M}}(\bar{t})$ of $b_{\mathrm{P}}(\bar{t})$ under the canonical map $\mathrm{B}\left(\mathrm{P} \otimes_{\mathbb{Q}} \mathbb{Q}_{p}\right) \rightarrow \mathrm{B}\left(\mathrm{M} \otimes_{\mathbb{Q}} \mathbb{Q}_{p}\right)$, which is in turn 
determined by the composition $\bar{t} \rightarrow W^{0} \rightarrow Z$. (Since the two outer graded pieces $T_{\mathrm{j}, \bar{t}}\left[p^{\infty}\right]$ and $\left(Y_{\mathrm{j}} \otimes_{\mathbb{Z}}\left(\mathbb{Q}_{p} / \mathbb{Z}_{p}\right)\right)_{\bar{t}}$ are multiplicative type and étale, the essential data is the middle graded piece $B_{\mathrm{j}, \bar{t}}\left[p^{\infty}\right]$ (with its additional structures), which is determined by the induced $\bar{t} \rightarrow Z$ Z) On the other hand, since $\mathcal{O} \otimes_{\mathbb{Z}} \mathbb{Q}$ involves no factor of type $\mathrm{D}$, by [36, Lemma 1.4.3.3] and by the proof of [37, Lemma A.4.7], the middle graded piece $\mathbb{D}\left(B_{\mathrm{j}, \bar{t}}\left[p^{\infty}\right]\right) \otimes_{W(k(\bar{t}))} K_{\bar{t}}$ is determined by $\mathbb{D}\left(A_{\mathrm{j}, \bar{t}}\left[p^{\infty}\right]\right) \otimes_{W(k(\bar{t}))} K_{\bar{t}}$ (both with their additional structures), and therefore $b(\bar{t})$ also determines $b_{\mathrm{M}}(\bar{t})$.

For each $b \in \mathrm{B}\left(\mathrm{G} \otimes_{\mathbb{Z}} \mathbb{Q}_{p}\right)$, and for each $Z$ as above, let us define a locally closed subset $Z_{s}^{b}$ of $Z_{s}$ as follows: We define $Z_{s}^{b}$ to be empty either if $\left(\mathbf{X}_{\mathcal{H}}\right)_{s}^{b}$ is empty, or if $b$ is not the image of any $b_{\mathrm{P}} \in \mathrm{B}\left(\mathrm{P} \otimes_{\mathbb{Q}} \mathbb{Q}_{p}\right)$. Otherwise, we define $Z_{s}^{b}$ to be $Z_{s}^{b_{h}}$, the Newton stratum of $Z_{s}$ associated with $b_{h} \in \mathrm{B}\left(\mathrm{G}_{h} \otimes_{\mathbb{Q}} \mathbb{Q}_{p}\right)$, where $\left(b_{l}, b_{h}\right)$ is the image of $b_{\mathrm{M}}$ under $\mathrm{B}\left(\mathrm{M} \otimes_{\mathbb{Q}} \mathbb{Q}_{p}\right) \cong \mathrm{B}\left(\mathrm{G}_{l} \otimes_{\mathbb{Q}} \mathbb{Q}_{p}\right) \times \mathrm{B}\left(\mathrm{G}_{h} \otimes_{\mathbb{Q}} \mathbb{Q}_{p}\right)$. (For our purpose, $b_{l}$ is not important, because it parameterizes the torus parts of degenerations.)

By the explanations above, for each $b \in \mathrm{B}\left(\mathrm{G} \otimes_{\mathbb{Z}} \mathbb{Q}_{p}\right)$ as above, and for each $W^{0}$ as in Proposition 2.1.3, the pullback of $Y:=\left(\mathbf{X}_{\mathcal{H}}\right)_{s}^{b}$ to $\left(W^{0}\right)_{s}$ coincides with the pullback of $Y_{Z}^{\natural}:=Z_{s}^{b}$. Thus, $Y$ is a well-positioned subset. The remaining assertions in the proposition are then self-explanatory.

REMARK 3.3.11. When $C \rightarrow \mathrm{Z}$ is reduced at some level $\mathcal{H}$, so that $\left(\mathrm{X}_{\mathcal{H}}\right)_{s}^{b}$ is a well-positioned subscheme of $\left(\mathrm{X}_{\mathcal{H}}\right)_{s}$ by Proposition 3.3.9, for each $b \in \mathrm{B}\left(\mathrm{G} \otimes_{\mathbb{Z}}\right.$ $\left.\mathbb{Q}_{p}\right)$, the pullback of $\left(\mathbf{X}_{\mathcal{H}}\right)_{s}^{b}$ to $\left(\mathbf{X}_{\mathcal{H}^{\prime}}\right)_{s}$ for each higher level $\mathcal{H}^{\prime} \subset \mathcal{H}$ is a wellpositioned subscheme of $\left(\mathrm{X}_{\mathcal{H}^{\prime}}\right)_{s}$, by Proposition 2.4.2, which underlies the same subset as $\left(\mathbf{X}_{\mathcal{H}^{\prime}}\right)_{s}^{b}$. (See Remark 3.2.9 for a similar consideration.) Then we can pullback from such a bottom level and obtain well-positioned subschemes over the Newton strata in all higher levels. Similar statements are true for pullbacks of $\left(\mathrm{X}_{\mathcal{H}}\right)_{s}^{v}$ to higher levels, for each $v \in \mathcal{N}$.

3.4. Oort central leaves and their pullbacks. In this subsection, we shall consider only $\mathbf{X}_{\mathcal{H}} \rightarrow \mathrm{S}$ in the following special case of Case $(\mathrm{Nm})$ : Suppose $p$ is a good prime (as in [36, Definition 1.4.1.1]) for the integral PEL datum $(\mathcal{O}, \star, L$, $\langle\cdot, \cdot\rangle, h_{0}$ ) in Assumption 2.1.1 (which we have insisted to satisfy [36, Condition 1.4.3.10]). Consider the trivial collection $\mathrm{J}=\left\{\mathrm{j}_{0}\right\}$ with

$$
\left\{\left(g_{\mathrm{j}_{0}}, L_{\mathrm{j}_{0}},\langle\cdot, \cdot\rangle_{\mathrm{j}_{0}}\right)\right\}=\{(1, L,\langle\cdot, \cdot\rangle)\}
$$

as in [38, Example 2.3]. Let $\mathcal{H}$ be any neat open compact subgroup of $\mathrm{G}(\hat{\mathbb{Z}})$. Let $\mathcal{H}^{p}$ denote the image of $\mathcal{H}$ under the canonical homomorphism $\mathrm{G}(\hat{\mathbb{Z}}) \rightarrow \mathrm{G}\left(\hat{\mathbb{Z}}^{p}\right)$, 
and let

$$
\mathcal{H}_{0}:=\mathcal{H}^{p} \mathrm{G}\left(\mathbb{Z}_{p}\right) .
$$

Since $p$ is a good prime for $\left(\mathcal{O}, \star, L,\langle\cdot, \cdot\rangle, h_{0}\right)$, we have a good reduction integral model $\mathrm{M}_{\mathcal{H}^{p}} \rightarrow \operatorname{Spec}\left(\mathcal{O}_{F_{0},(p)}\right)$ as in [36, Section 1.4.1]. By [36, Proposition 1.4.4.3], the canonical morphism $\mathrm{M}_{\mathcal{H}_{0}} \rightarrow \mathrm{M}_{\mathcal{H}^{p}} \otimes_{\mathbb{Z}} \mathbb{Q}$ is an open and closed immersion. Since the schemes $\overrightarrow{\mathrm{M}}_{\mathcal{H}_{0}}$ and $\overrightarrow{\mathrm{M}}_{\mathcal{H}}$ over $\overrightarrow{\mathrm{S}}_{0}=\operatorname{Spec}\left(\mathcal{O}_{F_{0},(p)}\right)$ in [38, Proposition 6.1] are independent of the auxiliary choices, by taking $\mathrm{M}_{\mathcal{H}}{ }^{p}$ as an auxiliary good reduction model, we have an open and closed immersion $\overrightarrow{\mathrm{M}}_{\mathcal{H}_{0}} \hookrightarrow \mathrm{M}_{\mathcal{H}^{p}}$, and we can take $\overrightarrow{\mathrm{M}}_{\mathcal{H}}$ to be the normalization of $\mathrm{M}_{\mathcal{H}^{p}}$ under the composition $\mathrm{M}_{\mathcal{H}} \rightarrow \mathrm{M}_{\mathcal{H}_{0}} \rightarrow \overrightarrow{\mathrm{M}}_{\mathcal{H}_{0}} \hookrightarrow \mathrm{M}_{\mathcal{H}^{p}}$ of canonical morphisms. Then we take $X_{\mathcal{H}} \rightarrow \mathrm{S}$ (respectively $\mathrm{X}_{\mathcal{H}_{0}} \rightarrow \mathrm{S}$ ) to be the pullback of $\overrightarrow{\mathrm{M}}_{\mathcal{H}} \rightarrow \overrightarrow{\mathrm{S}}_{0}$ (respectively $\overrightarrow{\mathrm{M}}_{\mathcal{H}_{0}} \rightarrow \overrightarrow{\mathrm{S}}_{0}$ ), which carries the pullback $(A, \lambda, i)$ of the tautological $\left(\vec{A}_{\mathrm{j}_{0}}, \vec{\lambda}_{\mathrm{j}_{0}}, \vec{i}_{\mathrm{j}_{0}}\right)$ over $\overrightarrow{\mathrm{M}}_{\mathcal{H}}$ (respectively $\left.\overrightarrow{\mathrm{M}}_{\mathcal{H}_{0}}\right)$.

Let $\mathrm{T}=\bar{s} \rightarrow \mathrm{S}$ be a geometric point above the special point $s=\operatorname{Spec}(k) \rightarrow \mathrm{S}$ of residue characteristic $p>0$. Let $\left(\mathbf{X}, \lambda_{\mathbf{X}}, i_{\mathbf{X}}\right)$ denote any Barsotti-Tate group $\mathbf{X}$ over $\bar{s}$, with a quasipolarization $\lambda_{\mathbf{X}}$ and an $\mathcal{O} \otimes_{\mathbb{Z}} \mathbb{Z}_{p}$-endomorphism structure $i_{\mathbf{X}}$ compatible with $\lambda_{\mathbf{x}}$. By [66, Theorem 3.3] for the case of quasipolarized BarsottiTate groups without additional structures, by the fact that there are only finitely many $\mathcal{O} \otimes_{\mathbb{Z}} \mathbb{Z}_{p}$-endomorphism structures over each polarized abelian scheme (see [36, Proposition 1.3.3.7]), and by (3) and (4) of Proposition 3.3.1, we obtain the following:

Proposition 3.4.1. Let $k_{0}$ and $\left(\mathbf{X}, \lambda_{\mathbf{X}}, i_{\mathbf{X}}\right)$ be as above.

(1) There is a locally closed subset

$$
\mathcal{C}_{\left(\mathbf{X}, \lambda_{\mathbf{X}}, i_{\mathbf{X}}\right)}\left(\left(\mathbf{X}_{\mathcal{H}}\right)_{\bar{s}}\right)
$$

of $\left(\mathrm{X}_{\mathcal{H}}\right)_{\bar{s}}$ containing all points $t \in\left(\mathrm{X}_{\mathcal{H}}\right)_{\bar{s}}$ such that there exists some geometric point $\bar{t}=\operatorname{Spec}(k(\bar{t})) \rightarrow\left(\mathrm{X}_{\mathcal{H}}\right)_{\bar{s}}$ above $t \in\left(\mathrm{X}_{\mathcal{H}}\right)_{\bar{s}}$ such that the triple $\left(A_{\bar{t}}\left[p^{\infty}\right], \lambda_{\bar{t}}, i_{\bar{t}}\right)$ over $\bar{t}$ defined by the pullback $\left(A_{\bar{t}}, \lambda_{\bar{t}}, i_{\bar{t}}\right)$ of the tautological triple over $\mathbf{X}_{\mathcal{H}}$ is isomorphic to the pullback of $\left(\mathbf{X}, \lambda_{\mathbf{X}}, i_{\mathbf{X}}\right)$.

(2) Consider the $b\left(\mathbf{X}, \lambda_{\mathbf{X}}, i_{\mathbf{X}}\right) \in \mathrm{B}\left(\mathrm{G} \otimes_{\mathbb{Z}} \mathbb{Q}_{p}\right)$ defined by the (rational) covariant Dieudonné module $\mathbb{D}(\mathbf{X}) \otimes_{W\left(k_{0}\right)} \operatorname{Frac}\left(W\left(k_{0}\right)\right)$ and its additional structures induced by $\lambda_{\mathbf{X}}$ and $i_{\mathbf{X}}$. Then $\mathcal{C}_{\left(\mathbf{X}, \lambda_{\mathbf{X}}, i_{\mathbf{X}}\right)}\left(\left(\mathbf{X}_{\mathcal{H}}\right)_{\bar{s}}\right)$ is a closed subset, called the Oort central leaf, of the Newton stratum $\left(\mathbf{X}_{\mathcal{H}}\right)_{\bar{s}}^{b\left(\mathbf{X}, \lambda_{\mathbf{X}}, i_{\mathbf{X}}\right)}$.

Proposition 3.4.2. Let $\bar{s}=\operatorname{Spec}(\bar{k})$ and $\left(\mathbf{X}, \lambda_{\mathbf{X}}, i_{\mathbf{X}}\right)$ be as above. The locally closed subset $\mathrm{Y}:=\mathcal{C}_{\left(\mathbf{X}, \lambda_{\mathbf{X}}, i_{\mathbf{X}}\right)}\left(\left(\mathbf{X}_{\mathcal{H}}\right)_{\bar{s}}\right)$ of $\left(\mathbf{X}_{\mathcal{H}}\right)_{\bar{s}}$ is a well-positioned subset as in 
Definition 2.2.1, which is associated with some collection $Y^{\natural}=\left\{Y_{Z}^{\natural}\right\}_{Z}$ such that $Y_{Z}^{\natural}$ is either the empty subset or some Oort central leaf of $Z_{\bar{s}}$ (whose precise definition will be made clear in the proof), for each $\mathrm{Z}$. With its reduced subscheme structure, $\mathcal{C}_{\left(\mathbf{X}, \lambda_{\mathbf{X}}, i_{\mathbf{X}}\right)}\left(\left(\mathrm{X}_{\mathcal{H}}\right)_{\bar{s}}\right)$ admits the partial minimal and toroidal compactifications $\mathrm{Y}^{\mathrm{min}}$ and $\mathrm{Y}_{\Sigma}^{\text {tor }}$ as in Definition 2.3.1 and Theorem 2.3.2, which we abusively denote by $\mathcal{C}_{\left(\mathbf{X}, \lambda_{\mathbf{X}}, i_{\mathbf{X}}\right)}\left(\left(\mathbf{X}_{\mathcal{H}}^{\min }\right)_{\bar{s}}\right)$ and $\mathcal{C}_{\left(\mathbf{X}, \lambda_{\mathbf{X}}, i_{\mathbf{X}}\right)}\left(\left(\mathbf{X}_{\mathcal{H}, \Sigma}^{\text {tor }}\right)_{\bar{s}}\right)$, respectively. By Lemma 2.2.10, when $C \rightarrow Z$ is reduced (which is the case when $\mathcal{H}=\mathcal{H}_{0}$, in which case $C \rightarrow \mathbf{Z}$ is smooth, by [36, Theorem 6.4.1.1]), $\mathcal{C}_{\left(\mathbf{X}, \lambda_{\mathbf{X}}, i_{\mathbf{X}}\right)}$ (with its reduced subscheme structure) is also a well-positioned subscheme.

To show this, we need the following technical lemma, which will also be useful for the consideration in Section 3.5:

LEMMA 3.4.3. With the setting as in Lemma 3.2.6, but with $\mathrm{j}$ and $\mathrm{J}$ suppressed from the notation system, for each $n \geqslant 1$, consider the canonical geometric filtration

$$
\mathrm{W}_{-3, p^{n}}=0 \subset \mathrm{W}_{-2, p^{n}}=T_{\bar{t}}\left[p^{n}\right] \subset \mathrm{W}_{-1, p^{n}}=G_{\bar{t}}^{\natural}\left[p^{n}\right] \subset \mathrm{W}_{0, p^{n}}=A_{\bar{t}}\left[p^{n}\right]
$$

on $A_{\bar{t}}\left[p^{n}\right]$ defined by (3.2.7) and (3.2.8), which is symplectic with respect to the $\lambda_{\bar{t}}$-Weil pairing

$$
e^{\lambda_{\bar{t}}}: A_{\bar{t}}\left[p^{n}\right] \times A_{\bar{t}}\left[p^{n}\right] \rightarrow \boldsymbol{\mu}_{p^{n}, \bar{t}}
$$

(which is perfect because $\lambda_{\bar{t}}$ is of degree prime to $p$, under the assumption that $p$ is good for $\left.\left(\mathcal{O}, \star, L,\langle\cdot, \cdot\rangle, h_{0}\right)\right)$ in the sense that $\mathrm{W}_{-2, p^{n}}$ and $\mathrm{W}_{-1, p^{n}}$ are the annihilators of each other.

Then there exists a noncanonical splitting

$$
\varsigma_{p^{n}}: \operatorname{Gr}_{p^{n}}^{\mathrm{W}}=T_{\bar{t}}\left[p^{n}\right] \oplus B_{\bar{t}}\left[p^{n}\right] \oplus\left(Y / p^{n} Y\right)_{\bar{t}} \stackrel{\sim}{\rightarrow} A_{\bar{t}}\left[p^{n}\right]
$$

of the filtration $\mathrm{W}_{p^{n}}$ which respects the canonical pairings in the sense that, for each $\left(x_{-2}, x_{-1}, x_{0}\right)$ and $\left(y_{-2}, y_{-1}, y_{0}\right)$ in $\mathrm{Gr}_{p^{n}}^{\mathrm{W}}$, we have

$$
\begin{aligned}
& e^{\lambda_{\bar{t}}}\left(\varsigma_{p^{n}}\left(\left(x_{-2}, x_{-1}, x_{0}\right)\right), \varsigma_{p^{n}}\left(\left(y_{-2}, y_{-1}, y_{0}\right)\right)\right) \\
& \quad=e^{\lambda_{B, \bar{t}}}\left(x_{-1}, y_{-1}\right)+\left[e^{\phi}\left(x_{-2}, y_{0}\right)-e^{\phi}\left(y_{-2}, x_{0}\right)\right],
\end{aligned}
$$

where

$$
e^{\lambda_{B, \bar{t}}}: B_{\bar{t}}\left[p^{n}\right] \times B_{\bar{t}}\left[p^{n}\right] \rightarrow \mu_{p^{n}, \bar{t}}
$$

is the $\lambda_{B, \bar{t}}$-Weil pairing on the abelian part, and where

$$
e^{\phi}: T_{\bar{t}}\left[p^{n}\right] \times\left(Y / p^{n} Y\right)_{\bar{t}} \rightarrow \boldsymbol{\mu}_{p^{n}, \bar{t}}
$$

is the canonical pairing defined by $e^{\phi}(x, y)=x(\phi(y))=(\phi(y))(x)$ for all

$$
x \in T_{\bar{t}}\left[p^{n}\right] \cong \underline{\operatorname{Hom}}_{\bar{t}}\left(\left(X / p^{n} X\right)_{\bar{t}}, \boldsymbol{\mu}_{p^{n}, \bar{t}}\right)
$$


and $y \in\left(Y / p^{n} Y\right)_{\bar{t}}$, where $\phi: Y \rightarrow X$ is dual to the homomorphism $\lambda_{T, \bar{t}}: T_{\bar{t}} \rightarrow T_{\bar{t}}^{\vee}$ between the torus parts, induced by $\lambda_{\bar{t}}^{\natural}$ (which are perfect pairings, because $\lambda_{B, \bar{t}}$ and $\phi$ are of degree prime to $p$ since $\lambda_{\bar{t}}$ is). We may assume that $\zeta_{p^{n}}$ lifts to similar splittings $S_{p^{n^{\prime}}}$ (respecting the canonical pairings) for all $n^{\prime} \geqslant n$.

Proof. Since $W$ is flat over $\mathrm{S}$, there exists a complete discrete valuation ring $V$ with residue field $k(\bar{t})$ and with generic point $\eta$ of residue characteristic zero, together with a morphism $\operatorname{Spec}(V) \rightarrow W^{0}$ lifting the geometric point $\bar{t} \rightarrow W^{0}$. Let us denote the pullbacks to $\eta$ of $A$ by $A_{\eta}$, and so forth, with subscripts $\eta$.

Since $\eta$ is of residue characteristic zero, and since some level structure $\alpha_{\mathcal{H}}$ is defined over $\mathbf{X}_{\mathcal{H}} \otimes_{\mathbb{Z}} \mathbb{Q}$, up to replacing $V$ with a finite flat extension (with the same residue field), we may assume that there is an integral principal level- $p^{n}$ structure $\alpha_{p^{n}, \eta}: L / p^{n} L \stackrel{\sim}{\rightarrow} A_{\eta}\left[p^{n}\right]$ of type $\left(L \otimes_{\mathbb{Z}} \hat{\mathbb{Z}},\langle\cdot, \cdot\rangle\right)$ as in [36, Definition 1.3.6.2], whose pullback to some geometric point $\bar{\eta} \rightarrow \eta$ is the reduction modulo $p^{n}$ of some symplectic isomorphism $\hat{\alpha}_{\bar{\eta}}: L \otimes_{\mathbb{Z}} \hat{\mathbb{Z}} \stackrel{\sim}{\rightarrow} \mathrm{T} A_{\bar{\eta}}$. By pulling back the geometric filtration on $\mathrm{T} A_{\bar{\eta}}$ defined by (3.2.7) and (3.2.8) (cf. [36, Proposition 5.2.2.1]), we obtain a filtration $Z$ on $L \otimes_{\mathbb{Z}} \hat{\mathbb{Z}}$ (whose $\mathcal{H}$-orbit $Z_{\mathcal{H}}$ is associated with the cusp label $\left[\left(\Phi_{\mathcal{H}}, \delta_{\mathcal{H}}\right)\right]$ for the stratum $\left.Z\right)$, which admits a noncanonical splitting $\hat{\delta}: \mathrm{Gr}^{Z} \stackrel{\sim}{\rightarrow} L \otimes_{\mathbb{Z}} \hat{\mathbb{Z}}$ of $\mathcal{O} \otimes_{\mathbb{Z}} \mathbb{Z}_{p}$-modules which respects the induced pairings between the graded pieces. Hence, by using the above isomorphism $\hat{\alpha}_{\bar{\eta}}$, there is also a noncanonical splitting $\hat{\zeta}_{\bar{\eta}}: \mathrm{T}_{p} T_{\bar{t}} \oplus \mathrm{T}_{p} B_{\bar{t}} \oplus\left(Y \otimes_{\mathbb{Z}} \mathbb{Z}_{p}\right)_{\bar{\eta}} \stackrel{\sim}{\rightarrow} \mathrm{T}_{p} A_{\bar{\eta}}$, whose reduction modulo $p^{n}$ descends to a noncanonical splitting

$$
\varsigma_{p^{n}, \eta}: \mathrm{Gr}_{p^{n}}^{\mathrm{W}}=T_{\eta}\left[p^{n}\right] \oplus B_{\eta}\left[p^{n}\right] \oplus\left(Y / p^{n} Y\right)_{\eta} \stackrel{\sim}{\rightarrow} A_{\eta}\left[p^{n}\right],
$$

which are compatible with the $\mathcal{O} \otimes_{\mathbb{Z}} \mathbb{Z}_{p}$-module structures and respect the induced pairings between the grade pieces. (See [36, Section 5.2.2] for all of these.)

By [36, Lemma 1.2.4.4], since $p$ is a good prime, up to a change of coordinates on $L \otimes_{\mathbb{Z}} \mathbb{Z}_{p}$ which replaces the above splittings with some other choices, we may assume that $\zeta_{p^{n}, \eta}$ is symplectic in the sense that the condition (3.4.6) holds with $\eta$ replacing $\bar{t}$. Moreover, for each $n^{\prime} \geqslant n$, up to replacing $\eta$ with a point $\eta^{\prime}$ finite over it, we may assume that $\varsigma_{p^{n}, \eta}$ lifts to a similar splitting $S_{p^{n^{\prime}}, \eta^{\prime}}$ (respecting the canonical pairings). By [36, Proposition 5.2.3.3, or rather the proofs of Lemmas 5.2.3.1 and 5.2.3.2], since $\eta$ is the generic point of a complete discrete valuation ring $V$, the splitting $\zeta_{p^{n}, \eta}$ extends to a splitting over $V$ respecting the canonical pairings (with $V$ replacing $\eta$ in (3.4.5) and (3.4.6)), whose pullback to $\bar{t}$ gives the desired splitting $S_{p^{n}, \bar{t}}$ as in (3.4.5), satisfying the condition given by (3.4.6), which lifts to similar splittings $\varsigma_{p^{n^{\prime}}}$ (respecting the canonical pairings) for all $n^{\prime} \geqslant n$.

Proof of Proposition 3.4.2. In Lemma 3.4.3, by varying $n \geqslant 1$, the isomorphism class of $\left(A_{\bar{t}}\left[p^{\infty}\right], \lambda_{\bar{t}}, i_{\bar{t}}\right)$ determines and is determined by the isomorphism classes 
of $(X, Y, \phi: Y \rightarrow X)$ and of $\left(B_{\bar{t}}\left[p^{\infty}\right], \lambda_{B, \bar{t}}, i_{B, \bar{t}}\right)$, which depends only on the composition $\bar{t} \rightarrow W^{0} \rightarrow$ Z. Therefore, for each $W^{0}$ as in Proposition 2.1.3, the pullback of $\mathrm{Y}=\mathcal{C}_{\left(\mathbf{X}, \lambda_{\mathbf{X}}, i_{\mathbf{X}}\right)}\left(\left(\mathbf{X}_{\mathcal{H}}\right)_{\bar{s}}\right)$ to $\left(W^{0}\right)_{\bar{s}}$ coincides with the pullback of some subset $Y_{Z}^{\natural}$ of $Z_{\bar{s}}$ which is either the empty subset, or some Oort central leaf, whose definition depends only on $\left(\mathbf{X}, \lambda_{\mathbf{X}}, i_{\mathbf{X}}\right)$. Hence, $\mathrm{Y}$ is a well-positioned subset. The remaining assertions in the proposition are then self-explanatory.

REMARK 3.4.7. Since $C \rightarrow \mathrm{Z}$ is reduced at level $\mathcal{H}_{0}$, so that $\mathcal{C}_{\left(\mathbf{X}, \lambda_{\mathbf{X}}, i_{\mathbf{X}}\right)}\left(\left(\mathrm{X}_{\mathcal{H}_{0}}\right)_{\bar{s}}\right)$ is a well-positioned subscheme of $\left(\mathbf{X}_{\mathcal{H}_{0}}\right)_{\bar{s}}$ by Proposition 3.4.2, the pullback of $\mathcal{C}_{\left(\mathbf{X}, \lambda_{\mathbf{X}}, i_{\mathbf{X}}\right)}\left(\left(\mathbf{X}_{\mathcal{H}_{0}}\right)_{\bar{s}}\right)$ to $\left(\mathbf{X}_{\mathcal{H}}\right)_{\bar{s}}$ is a well-positioned subscheme of $\left(\mathbf{X}_{\mathcal{H}}\right)_{\bar{s}}$, by Proposition 2.4.2, which underlies the same subset as $\mathcal{C}_{\left(\mathbf{X}, \lambda_{\mathbf{X}}, i_{\mathbf{X}}\right)}\left(\left(\mathbf{X}_{\mathcal{H}}\right)_{\bar{s}}\right)$. (See Remarks 3.2.9 and 3.3.11 for similar considerations.) Then we can pullback from $\mathcal{H}_{0}$ and obtain well-positioned subschemes over the Oort central leaves in all higher levels.

3.5. Ekedahl-Oort strata and their pullbacks. In this subsection, we shall consider only the same kinds of $X_{\mathcal{H}} \rightarrow \mathrm{S}$ in Case (Nm) as in Section 3.4. Let $\mathrm{T} \rightarrow \mathrm{S}$ be the special point $s=\operatorname{Spec}(k) \rightarrow \mathrm{S}$ of residue characteristic $p>0$. Consider any geometric point $\bar{t}=\operatorname{Spec}(k(\bar{t})) \rightarrow\left(\mathbf{X}_{\mathcal{H}}\right)_{s}$ above a point $t \in\left(\mathbf{X}_{\mathcal{H}}\right)_{s}$, which defines by pullback a triple $\left(A_{\bar{t}}, \lambda_{\bar{t}}, i_{\bar{t}}\right)$, and hence also a truncated BarsottiTate group $\left(A_{\bar{t}}\left[p^{n}\right], \lambda_{\bar{t}}, i_{\bar{t}}\right)$ with the induced quasipolarization and endomorphism structure, for each integer $n \geqslant 1$. When $n=1$, the isomorphism class of the triple $\left(A_{\bar{t}}[p], \lambda_{\bar{t}}, i_{\bar{t}}\right)$ is classified by the isomorphism class of the associated $F$-zip with additional structures. Concretely, this $F$-zip is $\left(M_{\bar{t}}, C_{\bar{t}}, D_{\bar{t}}, \varphi_{0, \bar{t}}, \varphi_{1, \bar{t}}\right)$, where $M_{\bar{t}}:=H_{1}^{\mathrm{dR}}\left(A_{\bar{t}} / \bar{t}\right):=H_{\mathrm{dR}}^{1}\left(A_{\bar{t}} / \bar{t}\right)^{\vee}$ is equipped with its two maximal totally isotropic submodules

$$
C_{\bar{t}}:=H^{0}\left(A_{\bar{t}}, \Omega_{A_{\bar{t}} / \bar{t}}^{1}\right)^{\perp}=\operatorname{ker}\left(H_{1}^{\mathrm{dR}}\left(A_{\bar{t}} / \bar{t}\right) \rightarrow \operatorname{Lie}_{A_{\bar{t}} / \bar{t}}\right)
$$

and

$$
D_{\bar{t}}:=\left(H^{1}\left(A_{\bar{t}}, \mathscr{H}^{0}\left(\Omega_{A_{\bar{t}} / \bar{t}}^{\bullet}\right)\right)\right)^{\perp}
$$

given by the Hodge filtration and the conjugate filtration, respectively, together with the isomorphisms $\varphi_{0, \bar{t}}:\left(M_{\bar{t}} / C_{\bar{t}}\right)^{(p)} \stackrel{\sim}{\rightarrow} D_{\bar{t}}$ and $\varphi_{1, \bar{t}}: C_{\bar{t}}^{(p)} \stackrel{\sim}{\rightarrow} M_{\bar{t}} / D_{\bar{t}}$ induced by the Cartier isomorphism, as in [83, Section 3.1] (which is dual to the construction in [57, Section 7.5]). As explained in [83, Example 3.2], $C_{\bar{t}}=\operatorname{ker}(F)$ and $D_{\bar{t}}=\operatorname{ker}(V)$, where the $\left(\sigma_{\bar{t}}\right.$-linear) Frobenius $F: M_{\bar{t}} \rightarrow M_{\bar{t}}$ and $\left(\sigma_{\bar{t}}^{-1}\right.$ linear) Verschiebung $V: M_{\bar{t}} \rightarrow M_{\bar{t}}$ are the respective reductions modulo $p$ of the corresponding $F$ and $V$ of the covariant Dieudonné module $\mathbb{D}\left(A_{\bar{t}}\left[p^{\infty}\right]\right.$ ) (which can be canonically identified with the dual of $\left.H_{\text {crys }}^{1}\left(A_{\bar{t}} / W(k(\bar{t}))\right)\right)$. 
For simplicity, assume that $\mathcal{O} \otimes_{\mathbb{Z}} \mathbb{Q}$ involves no factor of type $\mathrm{D}$, in the sense of [36, Definition 1.2.1.15], so that (any pullback of) $G \otimes_{\mathbb{Z}} \mathbb{Q}$ is connected. By [83, Sections 3.1 and 5.1-5.3, Theorems 7.1 and 10.1, Corollary 10.2, and Proposition 10.3] (and the references there to earlier works) for the case $\mathcal{H}=\mathcal{H}_{0}$ (with hyperspecial level at $p$ ), and by pulling back the locally closed strata to higher levels $\mathcal{H} \subset \mathcal{H}_{0}$, we obtain the following:

\section{PROPOSITION 3.5.1.}

(1) The isomorphism class of $F$-zips over $k(\bar{t})$ with additional structures (in the precise sense described in [83, Definition 3.1]) is classified by elements of the subset $W^{\mathrm{M}_{0}}$ of $W$ of minimal Weyl length representatives of $W_{\mathrm{M}_{0}} \backslash W$, where $W$ is the Weyl group of $\mathrm{G} \otimes_{\mathbb{Z}} \overline{\mathbb{F}}_{p}$, where $\overline{\mathbb{F}}_{p}$ is some algebraic closure of $\mathbb{F}_{p}$, and where $W_{\mathrm{M}_{0}}$ is the Weyl group of a Levi subgroup $\mathrm{M}_{0}$ of a parabolic subgroup $\mathrm{P}_{0}$ of $\mathrm{G} \otimes_{\mathbb{Z}} \overline{\mathbb{F}}_{p}$ defined up to conjugacy by $h_{0}$ (or rather by the $p$ adic version of $[\mu]$ determined by $h_{0}$, as in [45, Section 6.1]). In particular, there is an element $w(\bar{t}) \in W^{\mathrm{M}_{0}}$ associated with the above $F$-zip $\left(M_{\bar{t}}\right.$, $\left.C_{\bar{t}}, D_{\bar{t}}, \varphi_{0, \bar{t}}, \varphi_{1, \bar{t}}\right)$ with additional structures induced by $\lambda_{\bar{t}}$ and $i_{\bar{t}}$, which depends only on the image $t$ of $\bar{t} \rightarrow\left(\mathrm{X}_{\mathcal{H}}\right)_{s}$. Thus, it is unambiguous to write $w(t):=w(\bar{t})$.

(2) There is a partial ordering $\leqslant$ on the set $W^{\mathrm{M}_{0}}$ (see [73, Corollary 6.3]) such that, for each $w \in W^{\mathrm{M}_{0}}$, the subset $\left\{t \in\left(\mathbf{X}_{\mathcal{H}}\right)_{s}: w(t) \leqslant w\right\}$ of $\left(\mathbf{X}_{\mathcal{H}}\right)_{s}$ is closed, and so the subset

$$
\left(\mathbf{X}_{\mathcal{H}}\right)_{s}^{w}:=\left\{t \in\left(\mathbf{X}_{\mathcal{H}}\right)_{s}: w(t)=w\right\}
$$

of $\left(\mathrm{X}_{\mathcal{H}}\right)_{s}$ is locally closed. Hence, we have a set-theoretic disjoint union

$$
\left(\mathbf{X}_{\mathcal{H}}\right)_{s}=\coprod_{w \in W^{\mathrm{M}_{0}}}\left(\mathbf{X}_{\mathcal{H}}\right)_{s}^{w}
$$

(3) When $\mathcal{H}=\mathcal{H}_{0}$, the disjoint union (3.5.2) is a stratification in the sense that the closure of $\left(\mathbf{X}_{\mathcal{H}_{0}}\right)_{s}^{w}$ is $\left\{t \in\left(\mathbf{X}_{\mathcal{H}_{0}}\right)_{s}: w(t) \leqslant w\right\}=\bigcup_{w^{\prime} \leqslant w}\left(\mathbf{X}_{\mathcal{H}_{0}}\right)_{s}^{w^{\prime}}$, for each $w \in W^{\mathrm{M}_{0}}$. This is called the Ekedahl-Oort stratification of $\left(\mathrm{X}_{\mathcal{H}_{0}}\right)_{s}$ (see [55-57, 65, 84], and [83]). Moreover, each $\left(\mathrm{X}_{\mathcal{H}_{0}}\right)_{s}^{w}$ is equidimensional of dimension $l(w)$, and smooth (over $s$ ).

(4) The assignment of F-zips with additional structures works more generally over schemes over $s$ and defines a canonical morphism $\zeta:\left(\mathrm{X}_{\mathcal{H}}\right)_{s} \rightarrow \mathrm{Zip}$, where Zip abusively denotes the Artin stack over s of $F$-zips with additional structures. When $\mathcal{H}=\mathcal{H}_{0}$, the morphism $\zeta$ is smooth (by, for example, specializing [85, Theorem 3.1.2] to our setting here). 
REMARK 3.5.3. The disjoint union (3.5.2) might not be a stratification in general. Although it is indeed a stratification at level $\mathcal{H}_{0}$, the morphism $\left(\mathrm{X}_{\mathcal{H}}\right)_{s} \rightarrow\left(\mathrm{X}_{\mathcal{H}_{0}}\right)_{s}$ is not necessarily flat, and hence might not preserve the closure relations.

DEFINITION 3.5.4. By abuse of language, we shall still call each $\left(\mathbf{X}_{\mathcal{H}}\right)_{s}^{w}$ an Ekedahl-Oort stratum of $\left(\mathrm{X}_{\mathcal{H}}\right)_{s}$.

PROPOSITION 3.5.5. For each $w \in W^{\mathrm{M}_{0}}$, the locally closed subset $\mathrm{Y}:=\left(\mathrm{X}_{\mathcal{H}}\right)_{s}^{w}$ of $\left(\mathrm{X}_{\mathcal{H}}\right)_{s}$ is a well-positioned subset as in Definition 2.2.1, which is associated with some collection $Y^{\natural}=\left\{Y_{Z}^{\natural}\right\}_{Z}$ such that $Y_{Z}^{\natural}$ is either the empty subset or some Ekedahl-Oort stratum of $Z_{s}$ (whose precise definition will be made clear in the proof), for each $\mathbf{Z}$. With its reduced subscheme structure, $\left(\mathbf{X}_{\mathcal{H}}\right)_{s}^{w}$ admits the partial minimal and toroidal compactifications

$$
\left(\mathrm{X}_{\mathcal{H}}^{\min }\right)_{s}^{w}:=\mathrm{Y}^{\mathrm{min}} \quad \text { and } \quad\left(\mathrm{X}_{\mathcal{H}, \Sigma}^{\text {tor }}\right)_{s}^{w}:=\mathrm{Y}_{\Sigma}^{\text {tor }}
$$

as in Definition 2.3.1 and Theorem 2.3.2. By Lemma 2.2.10, when $C \rightarrow \mathrm{Z}$ is reduced (which is the case when $\mathcal{H}=\mathcal{H}_{0}$, in which case $C \rightarrow Z$ is smooth, by [36, Theorem 6.4.1.1]), $\left(\mathrm{X}_{\mathcal{H}}\right)_{s}^{w}$ (with its reduced subscheme structure) is also a well-positioned subscheme. The analogous statements are true if we consider the closed union $\bigcup_{w^{\prime} \leqslant w}\left(\mathbf{X}_{\mathcal{H}}\right)_{s}^{w^{\prime}}=\left\{t \in\left(\mathbf{X}_{\mathcal{H}}\right)_{s}: w(t) \leqslant w\right\}$ instead of $\left(\mathbf{X}_{\mathcal{H}}\right)_{s}^{w}$.

Proof. In Lemma 3.4.3, with $n=1$, the isomorphism class of $\left(A_{\bar{t}}[p], \lambda_{\bar{t}}, i_{\bar{t}}\right)$ determines and is determined by the isomorphism classes of $(X, Y, \phi: Y \rightarrow X)$ and of $\left(B_{\bar{t}}[p], \lambda_{B, \bar{t}}, i_{B, \bar{t}}\right)$, which depends only on the composition $\bar{t} \rightarrow W^{0} \rightarrow \mathrm{Z}$, because there is some splitting (3.4.5) which respects the pairings as in (3.4.6). Therefore, for each $w \in W^{\mathrm{M}_{0}}$, and for each $W^{0}$ as in Proposition 2.1.3, the pullback of $Y=\left(\mathbf{X}_{\mathcal{H}}\right)_{s}^{w}$ to $\left(W^{0}\right)_{s}$ coincides with the pullback of some subset $Y_{Z}^{\natural}$ of $Z_{s}$ which is either the empty subset, or some Ekedahl-Oort stratum, whose definition depends only on $w$ (and can be explicitly given in group-theoretic terms). Hence, $Y$ is a well-positioned subset. The remaining assertions in the proposition are then self-explanatory.

REMARK 3.5.6. Since $C \rightarrow Z$ is reduced at level $\mathcal{H}_{0}$, so that $\left(\mathbf{X}_{\mathcal{H}_{0}}\right)_{s}^{w}$ is a well-positioned subscheme of $\left(\mathbf{X}_{\mathcal{H}_{0}}\right)_{s}$ by Proposition 3.5.5, for each $w \in$ $W^{\mathrm{M}_{0}}$, the pullback of $\left(\mathrm{X}_{\mathcal{H}_{0}}\right)_{s}^{w}$ to $\left(\mathrm{X}_{\mathcal{H}}\right)_{s}$ is a well-positioned subscheme of $\left(\mathrm{X}_{\mathcal{H}}\right)_{s}$, by Proposition 2.4.2, which underlies the same subset as $\left(\mathbf{X}_{\mathcal{H}}\right)_{s}^{w}$. (See Remarks 3.2.9, 3.3.11, and 3.4.7 for similar considerations.) Then we can pullback from $\mathcal{H}_{0}$ and obtain well-positioned subschemes over the Ekedahl-Oort strata in all higher levels. 
Corollary 3.5.7. When $\mathcal{H}=\mathcal{H}_{0}$, the schemes $\left(\mathrm{X}_{\mathcal{H}, \Sigma}^{\text {tor }}\right)_{s}^{w}$ over $s$ are smooth (under the assumption in (4) of Proposition 2.1.2 that $\Sigma$ is smooth), for all $w \in W^{\mathrm{M}_{0}}$.

Proof. By Proposition 2.3.14, this follows from (3) of Proposition 3.5.1.

Corollary 3.5.8. The morphism $\zeta:\left(\mathrm{X}_{\mathcal{H}}\right)_{s} \rightarrow$ Zip in (4) of Proposition 3.5.1 (necessarily uniquely) extends to a morphism $\zeta^{\text {tor }}:\left(\mathrm{X}_{\mathcal{H}}^{\text {tor }}\right)_{s} \rightarrow \mathrm{Zip}$, and we have $\left(\mathrm{X}_{\mathcal{H}, \Sigma}^{\text {tor }}\right)_{s}^{w}=\left(\zeta^{\text {tor }}\right)^{-1}\left(z_{w}\right)$ as subsets of $\left(\mathbf{X}_{\mathcal{H}}^{\text {tor }}\right)_{s}$, for the same point $z_{w}$ of Zip such that $\left(\mathrm{X}_{\mathcal{H}}\right)_{s}^{w}=\zeta^{-1}\left(z_{w}\right)$, for each $w \in W^{\mathrm{M}_{0}}$. Moreover, $\zeta^{\text {tor }}$ is smooth (under the assumption in (4) of Proposition 2.1.2 that $\Sigma$ is smooth) when $\zeta$ is.

Proof. By an analogue of the argument of the proof of (7) of Theorem 2.3.2, by also approximating the finitely many objects and morphisms associated with $n=1$ (but ignoring those associated with $n^{\prime}>n$ ) in Lemma 3.4.3, we may assume that the étale morphisms $\bar{U} \rightarrow \mathrm{X}_{\mathcal{H}, \Sigma}^{\mathrm{tor}}$ and $\bar{U} \rightarrow E(\sigma) \times_{\operatorname{Spec}(\mathbb{Z})} C$ in Corollary 2.1.7 are adapted to all Ekedahl-Oort strata of $\left(\mathbf{X}_{\mathcal{H}}\right)_{s}$ in the sense (as in Definition 4.1.1 below) that, in the notation of Proposition 3.5.5, the pullbacks of $\left(\mathrm{X}_{\mathcal{H}, \Sigma}^{\text {tor }}\right)_{s}^{w}=\mathrm{Y}_{\Sigma}^{\text {tor }}$ and $\mathrm{Y}_{Z}^{\natural}$ coincides as subsets of $\bar{U}_{s}$, for each $w \in W^{\mathrm{M}_{0}}$. Then $\zeta:\left(\mathrm{X}_{\mathcal{H}}\right)_{s} \rightarrow$ Zip induces a morphism $U_{s} \rightarrow$ Zip, which factors through the morphisms $U_{s} \rightarrow E \times_{\operatorname{Spec}(\mathbb{Z})} C_{s} \rightarrow C_{s}$, by essentially the same argument as in the proof of Proposition 3.5.5. By composing the morphisms $\bar{U}_{s} \rightarrow E(\sigma) \times_{\operatorname{Spec}(\mathbb{Z})} C_{s} \rightarrow C_{s}$ with the induced morphism $C_{s} \rightarrow$ Zip, we obtain a (necessarily unique) extension $\bar{U}_{s} \rightarrow \mathrm{Zip}$, which is smooth when $U_{s} \rightarrow$ Zip is (cf. the paragraph preceding Proposition 2.3.13). By construction, the pullbacks of $\left(\mathrm{X}_{\mathcal{H}}\right)_{s}^{w}$ (respectively $\left.\left(\mathbf{X}_{\mathcal{H}, \Sigma}^{\text {tor }}\right)_{s}^{w}\right)$ and $z_{w}$ coincide as subsets of $U_{s}$ (respectively $\bar{U}_{s}$ ), for each $w \in W^{\mathrm{M}_{0}}$. Thus, by varying $\bar{U} \rightarrow \mathrm{X}_{\mathcal{H}, \Sigma}^{\text {tor }}$ and by étale descent, we obtain the desired extension $\zeta^{\text {tor }}:\left(\mathrm{X}_{\mathcal{H}}^{\text {tor }}\right)_{s} \rightarrow$ Zip of $\zeta$, with all the required properties.

3.6. Kottwitz-Rapoport strata and their pullbacks. In this subsection, we shall consider only $X_{\mathcal{H}} \rightarrow S$ in the following special cases of Cases $(\mathrm{Nm})$ and (Spl): As in [75, 6.2], assume that $\mathcal{O} \otimes_{\mathbb{Z}} \mathbb{Z}_{p}$ is a maximal order in $\mathcal{O} \otimes_{\mathbb{Z}} \mathbb{Q}_{p}$ (stable under $\star)$. Suppose that $\mathscr{L}$ is a (periodic and self-dual) multichain of $\left(\mathcal{O} \otimes_{\mathbb{Z}} \mathbb{Z}_{p}\right)$ lattices in $L \otimes_{\mathbb{Z}} \mathbb{Q}_{p}$, as in [75, Definition 3.4] and [43, Section 2.1]. Let $\mathcal{H}$ be any open compact subgroup of $\mathrm{G}\left(\mathbb{A}^{\infty}\right)$ such that its image $\mathcal{H}^{p}$ under the canonical homomorphism $\mathrm{G}(\hat{\mathbb{Z}}) \rightarrow \mathrm{G}\left(\hat{\mathbb{Z}}^{p}\right)$ is a neat (see [36, Definition 1.4.1.8]) open compact subgroup of $\mathrm{G}\left(\hat{\mathbb{Z}}^{p}\right)$, so that $\mathcal{H}$ is also neat, and such that the image $\mathcal{H}_{p}$ of $\mathcal{H}$ under the canonical homomorphism $\mathrm{G}(\hat{\mathbb{Z}}) \rightarrow \mathrm{G}\left(\mathbb{Z}_{p}\right)$ is contained in the connected stabilizer $\mathcal{H}_{0, p}$ of $\mathscr{L}$ (cf. [43, Definition 2.1.10 and Choices 2.2.10]). Let $\mathcal{H}_{0}:=\mathcal{H}^{p} \mathcal{H}_{0, p}$. 
As explained in [75, 3.2] and [43, Choices 2.2.9 and 2.2.10], there exists a finite subset $\mathscr{L}_{\mathrm{J}}=\left\{\Lambda_{\mathrm{j}}\right\}_{\mathrm{j} \in \mathrm{J}}$ of $\mathscr{L}$ such that an $\mathcal{O} \otimes_{\mathbb{Z}} \mathbb{Z}_{p}$-lattice $\Lambda$ in $L \otimes_{\mathbb{Z}} \mathbb{Q}_{p}$ belongs to $\mathscr{L}$ if and only if there exist some $r \in \mathbb{Z}$ and $\mathrm{j} \in \mathrm{J}$ such that $\Lambda=p^{r} \Lambda_{\mathrm{j}}$, and there exists a collection $\left\{\left(1, L_{\mathrm{j}},\langle\cdot, \cdot\rangle_{\mathrm{j}}\right)\right\}_{\mathrm{j} \in \mathrm{J}}$ (with the same index set) for the consideration in [38, Section 2] such that $\Lambda_{\mathrm{j}}=L_{\mathrm{j}} \otimes_{\mathbb{Z}} \mathbb{Z}_{p}$ in $L \otimes_{\mathbb{Z}} \mathbb{Q}_{p}$, such that $L_{\mathrm{j}} \otimes_{\mathbb{Z}} \hat{\mathbb{Z}}^{p}=L \otimes_{\mathbb{Z}} \hat{\mathbb{Z}}^{p}$, and such that $L_{\mathrm{j}_{0}}=p^{r_{0}} L$ for some $\mathrm{j}_{0} \in \mathrm{J}$ and some $r_{0} \in \mathbb{Z}$. Hence, $\left\{\left(1, L_{\mathrm{j}},\langle\cdot, \cdot\rangle_{\mathrm{j}}\right)\right\}_{\mathrm{j} \in \mathrm{J}}$ defines a flat integral model $\overrightarrow{\mathrm{M}}_{\mathcal{H}} \rightarrow \operatorname{Spec}\left(\mathcal{O}_{F_{0},(p)}\right)$ as in [38, Proposition 6.1].

Let $X_{\mathcal{H}} \rightarrow S$ (respectively $X_{\mathcal{H}_{0}} \rightarrow S$ ) be the pullback of $\overrightarrow{\mathrm{M}}_{\mathcal{H}} \rightarrow \overrightarrow{\mathrm{S}}_{0}$ (respectively $\overrightarrow{\mathrm{M}}_{\mathcal{H}_{0}} \rightarrow \overrightarrow{\mathrm{S}}_{0}$ ) in Case $(\mathrm{Nm})$, or of $\overrightarrow{\mathrm{M}}_{\mathcal{H}}^{\text {spl }} \rightarrow \operatorname{Spec}\left(\mathcal{O}_{K}\right)$ (respectively $\left.\overrightarrow{\mathrm{M}}_{\mathcal{H}_{0}}^{\text {spl }} \rightarrow \operatorname{Spec}\left(\mathcal{O}_{K}\right)\right)$ in Case (Spl). For compatibility with the setting in the theory of local models, suppose that $\mathrm{S} \rightarrow \operatorname{Spec}\left(\mathcal{O}_{F_{0},(p)}\right)$ factors through $\operatorname{Spec}\left(\mathcal{O}_{F_{0}, v}\right)$, where $\mathcal{O}_{F_{0}, v}$ is completion of $\mathcal{O}_{F_{0}}$ at some place $v \mid p$. In both cases, $\mathrm{X}_{\mathcal{H}} \rightarrow \mathrm{S}$ (respectively $\mathrm{X}_{\mathcal{H}_{0}} \rightarrow \mathrm{S}$ ) carries the pullback $\left\{\left(A_{\mathrm{j}}, \lambda_{\mathrm{j}}, i_{\mathrm{j}}\right)\right\}_{\mathrm{j} \in \mathrm{J}}$ of the tautological collection $\left\{\left(\vec{A}_{\mathrm{j}}, \vec{\lambda}_{\mathrm{j}}, \vec{i}_{\mathrm{j}}\right)\right\}_{\mathrm{j} \in \mathrm{J}}$ over $\overrightarrow{\mathrm{M}}_{\mathcal{H}}$ (respectively $\overrightarrow{\mathrm{M}}_{\mathcal{H}_{0}}$ ), which extends (up to periodicity) to an $\mathscr{L}$-set $(\underline{A}, \underline{\lambda}, \underline{\alpha})$ of abelian schemes (up to $\mathbb{Z}_{(p)}^{\times}$-isogeny, with additional structures), which is isomorphic to the pullback of the tautological one over the moduli $\mathrm{M}_{\mathcal{H}^{p}}^{\text {naive }}$ over $\operatorname{Spec}\left(\mathcal{O}_{F_{0}, v}\right)$ defined in [43, Definition 2.2.5], under a canonical morphism $\mathrm{X}_{\mathcal{H}} \rightarrow \mathrm{M}_{\mathcal{H}}^{\text {naive }}$.

Let $\mathrm{G}_{\mathscr{L}}$ denote the identity component of the group scheme over $\operatorname{Spec}\left(\mathbb{Z}_{p}\right)$ stabilizing the multichain $\mathscr{L}$, so that $\mathrm{G}_{\mathscr{L}}\left(\mathbb{Z}_{p}\right)=\mathcal{H}_{0, p}$. By [75, Sections 3 and 6] and [67, Theorem 2.2], $\mathrm{G}_{\mathscr{L}}$ is a smooth group scheme, and there is a $\mathrm{G}_{\mathscr{L}}$-torsor

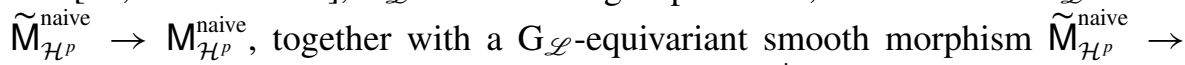
$M^{\text {naive }}$, which is of the same relative dimension as $\widetilde{\mathrm{M}}_{\mathcal{H}^{p}}^{\text {naive }} \rightarrow \mathrm{M}_{\mathcal{H}}^{\text {naive }}$, where $M^{\text {naive }}$ is the local model for $\mathrm{M}_{\mathcal{H}^{p}}^{\text {naive }}$, which is called the naive local model in later works such as [68]. Alternatively, there is a smooth morphism $\mathrm{M}_{\mathcal{H}^{p}}^{\text {naive }} \rightarrow\left[M^{\text {naive }} / \mathrm{G}_{\mathscr{L}}\right]$, which is of the same relative dimension as the smooth morphism $\mathrm{G}_{\mathscr{L}} \rightarrow \operatorname{Spec}\left(\mathbb{Z}_{p}\right)$, without having to explicitly mention $\tilde{\mathrm{M}}_{\mathcal{H}^{p}}^{\text {naive }}$. By composition with the canonical morphism $\mathrm{X}_{\mathcal{H}} \rightarrow \mathrm{M}_{\mathcal{H}^{p}}^{\text {naive }}$, we obtain a morphism $\varpi: \mathrm{X}_{\mathcal{H}} \rightarrow\left[M^{\text {naive }} / \mathrm{G}_{\mathscr{L}}\right]$ (which is not necessarily smooth or even flat).

Let $\mathrm{T}=\bar{s} \rightarrow \mathrm{S}$ be a geometric point above the special point $s=\operatorname{Spec}(k) \rightarrow \mathrm{S}$ of residue characteristic $p>0$. Consider the orbits $\bar{x}$ in

$$
\mathrm{KR}_{\bar{s}}:=M^{\text {naive }}(\bar{s}) / \mathrm{G}_{\mathscr{L}}(\bar{s})=\left[M^{\text {naive }} / \mathrm{G}_{\mathscr{L}}\right](\bar{s}) .
$$

Since the $\left(\mathrm{G}_{\mathscr{L}}\right)_{\bar{s}}$-orbits are locally closed in $\left(M^{\text {naive }}\right)_{\bar{s}}$ (see, for example, [76, Lemma 2.3.3]), the pullback of each $\bar{x} \in \mathrm{KR}_{\bar{s}}$ is a locally closed subset of $\mathrm{X}_{\mathcal{H}}(\bar{s})$ (in the induced Zariski topology). Hence, we have following: 
PROPOSITION 3.6.1.

(1) For any geometric $\bar{t} \rightarrow \bar{s}$, the canonical pullback map $\left[M^{\text {naive }} / \mathrm{G}_{\mathscr{L}}\right](\bar{s}) \rightarrow$ $\left[M^{\text {naive }} / \mathrm{G}_{\mathscr{L}}\right](\bar{t})$ is bijective. Consequently, there is a well-defined assignment $\varpi(\bar{t}) \in \mathrm{KR}_{\bar{s}}$ (still abusively denoted using $\varpi$ ) to each geometric point $\bar{t} \rightarrow$ $\left(\mathrm{X}_{\mathcal{H}}\right)_{\bar{s}}$, which induces a well-defined assignment $\varpi(t) \in \mathrm{KR}_{\bar{s}}$ to each point $t \in\left(\mathrm{X}_{\mathcal{H}}\right)_{\bar{s}}$.

(2) Let us equip $\mathrm{KR}_{\bar{s}}$ with the partial ordering $\leqslant$ such that $\bar{x}^{\prime} \leqslant \bar{x}$ exactly when the orbit $\bar{x}^{\prime}$ is contained in the closure of the orbit $\bar{x}$. For each $\bar{x} \in \mathrm{KR}_{\bar{s}}$, the subset $\left\{t \in\left(\mathrm{X}_{\mathcal{H}}\right)_{\bar{s}}: \varpi(t) \leqslant \bar{x}\right\}=\bigcup_{\bar{x}^{\prime} \leqslant \bar{x}}\left(\mathrm{X}_{\mathcal{H}}\right)_{\bar{s}}^{\bar{x}^{\prime}}$ of $\left(\mathrm{X}_{\mathcal{H}}\right)_{\bar{s}}$ is closed, and so the subset

$$
\left(\mathrm{X}_{\mathcal{H}}\right)_{\bar{s}}^{\bar{x}}:=\left\{t \in\left(\mathrm{X}_{\mathcal{H}}\right)_{\bar{s}}: \varpi(t)=\bar{x}\right\}
$$

of $\left(\mathrm{X}_{\mathcal{H}}\right)_{\bar{s}}$ is locally closed. Hence, we have a set-theoretic disjoint union

$$
\left(\mathrm{X}_{\mathcal{H}}\right)_{\bar{s}}=\coprod_{\bar{x} \in \mathrm{KR}_{\bar{s}}}\left(\mathrm{X}_{\mathcal{H}}\right)_{\bar{s}}^{\bar{x}} .
$$

REMARK 3.6.3. Since $\varpi: \mathrm{X}_{\mathcal{H}} \rightarrow\left[M^{\text {naive }} / \mathrm{G}_{\mathscr{L}}\right]$ is not necessarily flat, the disjoint union (3.6.2) might not be a stratification in general, in the sense that the closure of a stratum might not be a union of strata. Nevertheless, it is indeed a stratification when $\varpi$ is flat. This is the case when $\mathcal{H}=\mathcal{H}_{0}$ and when $M^{\text {naive }}$ happens to be flat over $\operatorname{Spec}\left(\mathbb{Z}_{p}\right)$ and normal, in which case the canonical morphism $\overrightarrow{\mathrm{M}}_{\mathcal{H}_{0}} \rightarrow \mathrm{M}_{\mathcal{H}^{p}}^{\text {naive }}$ is an open and closed immersion.

Definition 3.6.4. By abuse of language, we shall still call $\left(\mathrm{X}_{\mathcal{H}}\right)_{\bar{s}}^{\bar{x}}$ a KottwitzRapoport stratum of $\left(\mathrm{X}_{\mathcal{H}}\right)_{\bar{s}}$. (See [64, Section 3] and the introduction of [25].)

REMARK 3.6.5. Since the morphism $\varpi: \mathrm{X}_{\mathcal{H}} \rightarrow\left[M^{\text {naive }} / \mathrm{G}_{\mathscr{L}}\right]$ factors through $\left[M^{\text {loc }} / \mathrm{G}_{\mathscr{L}}\right] \rightarrow\left[M^{\text {naive }} / \mathrm{G}_{\mathscr{L}}\right]$ in both Cases $(\mathrm{Nm})$ and (Spl) (cf. [68, (15.4)]), it makes sense to also introduce Kottwitz-Rapoport strata based on the orbits in $\left[M^{\mathrm{loc}} / \mathrm{G}_{\mathscr{L}}\right](\bar{s})$. (In Case $(\mathrm{Spl})$, we can also consider $\left[M^{\mathrm{spl}} / \mathrm{G}_{\mathscr{L}}\right]$ instead of $\left[M^{\text {loc }} / \mathrm{G}_{\mathscr{L}}\right]$.) In what follows, all the results in Proposition 3.6.6, Remark 3.6.7, and Corollary 3.6.9 will remain correct if we replace the target of the morphism $\varpi: \mathrm{X}_{\mathcal{H}} \rightarrow\left[M^{\text {naive }} / \mathrm{G}_{\mathscr{L}}\right]$ with $\left[M^{\text {loc }} / \mathrm{G}_{\mathscr{L}}\right](\bar{s})$ (or with $\left[M^{\text {spl }} / \mathrm{G}_{\mathscr{L}}\right](\bar{s})$ in Case $(\mathrm{Spl}))$, and replace the definition of $\mathrm{KR}_{\bar{s}}$ accordingly.

Proposition 3.6.6. For each $\bar{x} \in \mathrm{KR}_{\bar{s}}$, the locally closed subset $\mathrm{Y}:=\left(\mathrm{X}_{\mathcal{H}}\right)_{\bar{s}}^{\bar{x}}$ of $\left(\mathrm{X}_{\mathcal{H}}\right)_{\bar{s}}$ is a well-positioned subset as in Definition 2.2.1, which is associated with some collection $Y^{\natural}=\left\{Y_{Z}^{\natural}\right\}_{Z}$ such that $Y_{Z}^{\natural}$ is either the empty subset or some Kottwitz-Rapoport stratum of $Z_{\bar{s}}$ (whose precise definition will be made clear in the proof), for each $\mathrm{Z}$. With its reduced subscheme structure, $\left(\mathrm{X}_{\mathcal{H}}\right)_{\bar{s}}^{\bar{x}}$ admits the 
partial minimal and toroidal compactifications $\left(\mathrm{X}_{\mathcal{H}}^{\min }\right)_{\bar{s}}^{\bar{x}}:=\mathrm{Y}^{\min }$ and $\left(\mathrm{X}_{\mathcal{H}, \Sigma}^{\text {tor }}\right)_{\bar{s}}^{\bar{x}}:=$ $\mathrm{Y}_{\Sigma}^{\mathrm{tor}}$ as in Definition 2.3.1 and Theorem 2.3.2. By Lemma 2.2.10, when $C \rightarrow \mathrm{Z}$ is reduced, $\left(\mathrm{X}_{\mathcal{H}}\right)_{\bar{s}}^{\bar{x}}$ (with its reduced subscheme structure) is also a well-positioned subscheme. The analogous statements are true if we consider the closed union $\bigcup_{\bar{x}^{\prime} \leqslant \bar{x}}\left(\mathrm{X}_{\mathcal{H}}\right)_{\bar{s}}^{\bar{x}^{\prime}}=\left\{t \in\left(\mathrm{X}_{\mathcal{H}}\right)_{\bar{s}}: \varpi(t) \leqslant \bar{x}\right\}$ instead of $\left(\mathrm{X}_{\mathcal{H}}\right)_{\bar{s}}^{\bar{x}}$.

Proof. As in the proof of Proposition 3.3.9, by Lemma 3.2.6, for each $\mathrm{j} \in \mathrm{J}$ and for each geometric point $\bar{t} \rightarrow W^{0}$, the Barsotti-Tate group $A_{\mathrm{j}, \bar{t}}\left[p^{\infty}\right]$ admits a filtration $0 \subset T_{\mathrm{j}, \bar{t}}\left[p^{\infty}\right] \subset G_{\mathrm{j}, \bar{t}}^{\natural}\left[p^{\infty}\right] \subset A_{\mathrm{j}, \bar{t}}\left[p^{\infty}\right]$ with graded pieces given by the (multiplicative-type) torus part $T_{\mathrm{j}, \bar{t}}\left[p^{\infty}\right]$, the abelian part $B_{\mathrm{j}, \bar{t}}\left[p^{\infty}\right]$, and the (étale) constant part $\left(Y_{\mathrm{j}} \otimes_{\mathbb{Z}}\left(\mathbb{Q}_{p} / \mathbb{Z}_{p}\right)\right)_{\bar{t}}$, compatibly equipped with quasipolarizations and endomorphism structures, and such filtrations are compatible with each other and with twists by $\sigma_{k(\bar{t})}$. Consequently, by considering the associated covariant Dieudonné modules, the kernel of

$$
V: \mathbb{D}\left(A_{\mathrm{j}, \bar{t}}^{\sigma_{\bar{t}}}\left[p^{\infty}\right]\right) \rightarrow \mathbb{D}\left(A_{\mathrm{j}, \bar{t}}\left[p^{\infty}\right]\right)
$$

determines and is determined by the kernel of

$$
V: \mathbb{D}\left(B_{\mathrm{j}, \bar{t}}^{\sigma_{\bar{t}}}\left[p^{\infty}\right]\right) \rightarrow \mathbb{D}\left(B_{\mathrm{j}, \bar{t}}\left[p^{\infty}\right]\right),
$$

where the superscripts $\sigma_{\bar{t}}$ denotes the pullback by $\sigma_{\bar{t}}$. Hence, $\varpi(\bar{t})$ depends only on the composition $\bar{t} \rightarrow W^{0} \rightarrow \mathbf{Z}$, because it is determined by the former kernels (for all $\mathrm{j} \in \mathrm{J}$ ) (cf. [25, the proof of Proposition 2.5.9, and Remark 2.5.11]), while the latter kernels (by definition) only depend on the abelian parts. Therefore, for each $\bar{x} \in \mathrm{KR}_{\bar{s}}$, and for each $W^{0}$ as in Proposition 2.1.3, the pullback of $Y:=\left(\mathrm{X}_{\mathcal{H}}\right)_{\bar{s}}^{\bar{x}}$ to $\left(W^{0}\right)_{\bar{s}}$ coincides with the pullback of some subset $Y_{Z}^{\natural}$ of $Z_{\bar{s}}$ which is either the empty subset, or some Kottwitz-Rapoport stratum, whose definition depends only on $\bar{x}$ (and can be explicitly given in group-theoretic terms). Hence, $\mathrm{Y}$ is a well-positioned subset. The remaining assertions in the proposition are then selfexplanatory.

REMARK 3.6.7. When $C \rightarrow \mathrm{Z}$ is reduced at some level $\mathcal{H}$, so that $\left(\mathrm{X}_{\mathcal{H}}\right)_{s}^{\bar{x}}$ is a well-positioned subscheme of $\left(\mathrm{X}_{\mathcal{H}}\right)_{\bar{s}}$ by Proposition 3.6.6, for each $\bar{x} \in \mathrm{KR}_{\bar{s}}$, the pullback of $\left(\mathrm{X}_{\mathcal{H}}\right)_{\bar{s}}^{\bar{x}}$ to $\left(\mathrm{X}_{\mathcal{H}^{\prime}}\right)_{\bar{s}}$ for each higher level $\mathcal{H}^{\prime} \subset \mathcal{H}$ is a well-positioned subscheme of $\left(\mathrm{X}_{\mathcal{H}^{\prime}}\right)_{\bar{s}}$, by Proposition 2.4.2, which underlies the same subset as $\left(\mathrm{X}_{\mathcal{H}^{\prime}}\right)_{\bar{s}}^{\bar{x}}$. (See Remarks 3.2.9, 3.3.11, 3.4.7, and 3.5.6 for similar considerations.) Then we can pullback from such a bottom level and obtain well-positioned subschemes over the Kottwitz-Rapoport strata in all higher levels.

COROLlaRY 3.6.8. For each $\bar{x} \in \mathrm{KR}_{\bar{s}}$, the scheme $\left(\mathrm{X}_{\mathcal{H}, \Sigma}^{\text {tor }}\right)_{\bar{s}}^{\bar{x}}$ is smooth over $\bar{s}$ (under the assumption in (4) of Proposition 2.1.2 that $\Sigma$ is smooth) when 
$\left(\mathrm{X}_{\mathcal{H}}\right)_{\bar{s}}^{\bar{x}}$ is, and the scheme $\bigcup_{\bar{x}^{\prime} \leqslant \bar{x}}\left(\mathrm{X}_{\mathcal{H}, \Sigma}^{\text {tor }}\right)_{\bar{s}}^{\bar{x}}$ is normal and Cohen-Macaulay when $\bigcup_{\bar{x}^{\prime} \leqslant \bar{x}}\left(\mathrm{X}_{\mathcal{H}}\right)_{\bar{s}}^{\bar{x}^{\prime}}$ is.

Proof. By Proposition 2.3.14, this follows from (3) of Proposition 3.5.1.

COROllary 3.6.9. The morphism $\varpi: \mathrm{X}_{\mathcal{H}}$ (necessarily uniquely) extends to a morphism $\varpi^{\text {tor }}: \mathrm{X}_{\mathcal{H}}^{\text {tor }} \rightarrow\left[M^{\text {naive }} / \mathrm{G}_{\mathscr{L}}\right]$, and we have $\left(\mathrm{X}_{\mathcal{H}, \Sigma}^{\text {tor }}\right)_{\bar{s}}^{\bar{x}}=\left(\varpi^{\text {tor }}\right)^{-1}(\bar{x})$ as subsets of $\left(\mathrm{X}_{\mathcal{H}}^{\text {tor }}\right)_{\bar{s}}$, for each $\bar{x} \in \mathrm{KR}_{\bar{s}}=\left[M^{\text {naive }} / \mathrm{G}_{\mathscr{L}}\right](\bar{s})$. Moreover, $\varpi^{\text {tor }}$ is smooth (under the assumption in (4) of Proposition 2.1.2 that $\Sigma$ is smooth) when $\varpi$ is.

Proof. Suppose that $\bar{s} \rightarrow s$ factors through some scheme of finite type over $s$ over which all the finitely many Kottwitz-Rapoport strata of $\left(\mathrm{X}_{\mathcal{H}}\right)_{\bar{s}}$ have models. As in the proof of Corollary 3.5.8, by also approximating these models, we may assume that the étale morphisms $\bar{U} \rightarrow \mathrm{X}_{\mathcal{H}, \Sigma}^{\text {tor }}$ and $\bar{U} \rightarrow E(\sigma) \times_{\operatorname{Spec}(\mathbb{Z})} C$ in Corollary 2.1.7 are adapted (as in Definition 4.1.1 below) to all KottwitzRapoport strata of $\left(\mathrm{X}_{\mathcal{H}}\right)_{\bar{s}}$, in the sense that, in the notation of Proposition 3.6.6, the pullback of $\left(\mathrm{X}_{\mathcal{H}, \Sigma}^{\text {tor }}\right)_{\bar{s}}^{\bar{x}}=\mathrm{Y}_{\Sigma}^{\text {tor }}$ to $\bar{U}_{\bar{s}}$ coincides with the pullback of $\mathrm{Y}_{Z}^{\natural}$, for each $w \in W^{\mathrm{M}_{0}}$. Then, by proceeding as in the proof of Corollary 3.5.8, with the proof of Proposition 3.5.5 replaced with that of Proposition 3.6.6 as an input, we obtain the desired extension $\varpi^{\text {tor }}$ of $\varpi$, with all the required properties.

EXAMPLE 3.6.10. Let us work in Case $(\mathrm{Nm})$, and replace $\varpi: \mathrm{X}_{\mathcal{H}} \rightarrow$ $\left[M^{\text {naive }} / \mathrm{G}_{\mathscr{L}}\right]$ with $\varpi: \mathrm{X}_{\mathcal{H}} \rightarrow\left[M^{\text {loc }} / \mathrm{G}_{\mathscr{L}}\right]$ as in Remark 3.6.5. Suppose that $p>2$ and $\mathrm{G} \otimes_{\mathbb{Z}} \mathbb{Q}_{p}$ splits over a tamely ramified extension of $\mathbb{Q}_{p}$, and that $\mathcal{H}=\mathcal{H}_{0}$ and $\mathcal{H}_{0, p}$ is the full stabilizer of the multichain $\mathscr{L}$ in $\mathrm{G}\left(\mathbb{Q}_{p}\right)$. Also, suppose that $\mathcal{O} \otimes_{\mathbb{Z}} \mathbb{Q}$ involves no factor of type $\mathrm{D}$, in the sense of [36, Definition 1.2.1.15], so that $G \otimes_{\mathbb{Z}} \mathbb{Q}_{p}$ is connected with simply connected derived group. Then it follows from [69, Theorem 1.2 and Section 8.2] and the construction in [38] (see Assumption 2.1.1) that $\varpi: \mathrm{X}_{\mathcal{H}} \rightarrow\left[M^{\text {loc }} / \mathrm{G}_{\mathscr{L}}\right]$ is smooth. Hence, by Corollary 3.6.9, $\varpi^{\text {tor }}: \mathrm{X}_{\mathcal{H}}^{\text {tor }} \rightarrow\left[M^{\text {loc }} / \mathrm{G}_{\mathscr{L}}\right]$ is also smooth. Moreover, it follows from [69, Theorem 1.1 and Section 9] that, for each $\bar{x} \in \mathrm{KR}_{\bar{s}}$, the scheme $\left(\mathrm{X}_{\mathcal{H}}\right)_{\bar{s}}^{\bar{x}}$ (respectively $\left.\bigcup_{\bar{x}^{\prime} \leqslant \bar{x}}\left(\mathrm{X}_{\mathcal{H}}\right)_{\bar{s}}^{\bar{x}^{\prime}}\right)$ is smooth over $s$ (respectively normal and Cohen-Macaulay). By Corollary 3.6.8, the scheme $\left(\mathrm{X}_{\mathcal{H}, \Sigma}^{\text {tor }}\right)_{\bar{s}}^{\bar{x}}$ (respectively $\left.\bigcup_{\bar{x}^{\prime} \leqslant \bar{x}}\left(\mathbf{X}_{\mathcal{H}, \Sigma}^{\text {tor }}\right)_{\bar{s}}^{\bar{x}^{\prime}}\right)$ has the same property.

REMARK 3.6.11. The philosophy behind our strategies in Sections 3.2-3.6 can be (very roughly) summarized as follows: Suppose that we have a stratification of a characteristic $p$ fiber of an integral model of a Shimura variety defined by pulling back a stratification of the stack of $p$-divisible groups, and that the formation of this latter stratification is insensitive to the étale parts of $p$-divisible groups. Then every stratum of the former stratification should be well positioned, and its 
partial toroidal and minimal compactifications should be stratified by pullbacks of strata of the stack of $p$-divisible groups (of smaller heights).

REMARK 3.6.12. Likewise, the Ekedahl-Kottwitz-Oort-Rapoport (EKOR) stratification introduced in [26, Section 6] should also be well positioned. We leave the details to the interested readers.

3.7. Supports of nearby cycles. In this subsection, we again consider all cases in Assumption 2.1.1. Consider any rational prime number $\ell \neq p$. Suppose $\Lambda=\mathbb{Q}_{\ell}$ or $\overline{\mathbb{Q}}_{\ell}$. Recall the notion of the supports of a $\Lambda$-perverse sheaf $\mathcal{F}$ over a scheme $X$ of finite type over a base field $k$ which we assume (for simplicity) to be either algebraically closed or finite (see [6, 2.2.14]). By [6, 4.3.1], $\mathcal{F}$ has a finite Jordan-Hölder filtration, indexed by some finite set $I$, with simple graded pieces. For each $i \in I$, the corresponding graded piece is isomorphic to some $J_{Y_{i}, ! *}\left(\mathcal{G}_{i}\left[\operatorname{dim}\left(Y_{i}\right)\right]\right)$, where $Y_{i}$ is a reduced irreducible closed subscheme of $X$, where $J_{Y_{i}}: Y_{i}^{0} \hookrightarrow X$ is an immersion from a smooth open dense subscheme $Y_{i}^{0}$ of $Y_{i}$, and where $\mathcal{G}_{i}$ is an irreducible lisse sheaf over $Y_{i}^{0}$.

DEFINITION 3.7.1. With the setting as above, we shall write

$$
\operatorname{Supp}(\mathcal{F})=\left\{Y_{i}: i \in I\right\},
$$

ignoring possible repetitions, and call $Y_{i}$ the supports of $\mathcal{F}$.

REMARK 3.7.2. The closed subschemes $Y_{i}$ are well determined for all $i \in I$, but their smooth open subschemes $Y_{i}^{0}$ are not: one can always replace $Y_{i}^{0}$ with an open dense subscheme. Since each lisse sheaf $\mathcal{G}_{i}$ over $Y_{i}^{0}$ is uniquely determined by its restriction to any open dense subscheme of $Y_{i}^{0}$, we abusively say that $\mathcal{G}_{i}$ is well determined, despite the fact that $Y_{i}^{0}$ is not.

Suppose $\mathcal{G}_{i}$ and $\mathcal{G}_{i^{\prime}}$ are irreducible lisse sheaves as above, with $i, i^{\prime} \in I$, which are defined over $Y_{i}^{0}$ and $Y_{i^{\prime}}^{0}$, respectively. We abusively consider them isomorphic if $Y_{i}^{0}$ and $Y_{i^{\prime}}^{0}$ have the same closure $Y_{i}=Y_{i^{\prime}}$ in $X$, and if the restrictions of $\mathcal{G}_{i}$ and $\mathcal{G}_{i^{\prime}}$ to $Y_{i}^{0} \cap Y_{i^{\prime}}^{0}$ are isomorphic to each other.

Definition 3.7.3. For each $Y \in \operatorname{Supp}(\mathcal{F})$, we denote by $\operatorname{Loc}_{Y}(\mathcal{F})$ the set of isomorphism classes of $\mathcal{G}_{i}$ as above.

By abuse of language, we shall freely shrink or increase the open subscheme $Y_{i}^{0}$ of $Y_{i}$ over which each such $\mathcal{G}_{i}$ is defined (see Remark 3.7.2). For $Y \in \operatorname{Supp}(\mathcal{F})$ and $\mathcal{G} \in \operatorname{Loc}_{Y}(\mathcal{F})$, we define $m(Y, \mathcal{G})$ to be the multiplicity of $J_{Y, ! *}(\mathcal{G}[\operatorname{dim}(Y)])$ 
in any Jordan-Hölder sequence of $\mathcal{F}$. Then we have

$$
[\mathcal{F}]=\sum_{Y \in \operatorname{Supp}(\mathcal{F})} \sum_{\mathcal{G} \in \operatorname{Loc}_{Y}(\mathcal{F})} m(Y, \mathcal{G})\left[J_{Y, ! *}(\mathcal{G}[\operatorname{dim}(Y)])\right]
$$

in the Grothendieck group of perverse sheaves over $X$.

REMARK 3.7.5. If $\mathcal{V}$ is a nowhere zero lisse sheaf over $X$, we have canonically $\operatorname{Supp}(\mathcal{F})=\operatorname{Supp}(\mathcal{F} \otimes \mathcal{V})$. For each support $Y$, we have a canonical multivalued map $\operatorname{Loc}_{Y}(\mathcal{F}) \rightarrow \operatorname{Loc}_{Y}(\mathcal{F} \otimes \mathcal{V})$ sending $\mathcal{G}$ to the irreducible Jordan-Hölder constituents of $\mathcal{G} \otimes \mathcal{V}$. For example, for any flat model $\mathrm{X}_{\mathcal{H}} \rightarrow \mathrm{S}$ of relative dimension $d$ considered in Assumption 2.1.1, for any nonzero étale sheaf $\mathcal{V}_{\xi}$ as in [45, Proposition 3.2], which is defined over all of $X_{\mathcal{H}}$ (under the assumption that $\ell \neq p$ ), and for any $i: s=\operatorname{Spec}(k) \rightarrow \mathrm{S}, j: \eta=\operatorname{Spec}(K) \rightarrow \mathrm{S}$, $\bar{i}: \bar{s}=\operatorname{Spec}(\bar{k}) \rightarrow \overline{\mathrm{S}}$, and $\bar{j}: \bar{\eta}=\operatorname{Spec}(\bar{K}) \rightarrow \overline{\mathrm{S}}$ as in [45, Section 5.1], the supports of $R \Psi_{\mathrm{X}_{\mathcal{H}}}(\Lambda[d])$ and $R \Psi_{\mathrm{X}_{\mathcal{H}}}\left(\mathcal{V}_{\xi}[d]\right)$ (which are perverse sheaves on $\left(\mathrm{X}_{\mathcal{H}}\right)_{\bar{s}}$ by $[27,4.5])$ coincide. Therefore, for the sake of simplicity, we shall often focus on the trivial coefficient case.

REMARK 3.7.6. In Cases ( $\mathrm{Nm})$ and $(\mathrm{Spl})$, when $\mathcal{H}$ has a high level at $p$, we have no geometric information about the 'bad reduction' of $\mathrm{X}_{\mathcal{H}} \rightarrow \mathrm{S}$. Nevertheless, by definition, the supports of $R \Psi_{\mathrm{X}_{\mathcal{H}}}(\Lambda[d])$ still give important topological invariants of this bad reduction. We show that these supports have good properties near the boundary of $\left(\mathbf{X}_{\mathcal{H}}\right)_{\bar{s}}$ (or, more precisely, near the boundary of any $\left.\left(\mathbf{X}_{\mathcal{H}, \Sigma}^{\text {tor }}\right)_{\bar{s}}\right)$, even though we do not know their precise shape in the interior.

Let us introduce the following:

ASSUMPTION 3.7.7. All the proper surjective morphisms $C \rightarrow Z$ (in Proposition 2.1.2) are smooth and has (nonempty) connected geometric fibers.

REMARK 3.7.8. Assumption 3.7.7 holds, for example, in the contexts of Remark 2.1.8 and Lemmas 2.1.9 and 2.1.10.

Lemma 3.7.9. Suppose that Assumption 3.7.7 holds, and that $\bar{U} \rightarrow \mathrm{X}_{\mathcal{H}, \Sigma}^{\text {tor }}$ and $\bar{U} \rightarrow E(\sigma) \times_{\operatorname{Spec}(\mathbb{Z})} C$ are étale morphisms as in Corollary 2.1.7, with $U$ the common preimage of $\mathrm{X}_{\mathcal{H}}$ and $E \times_{\operatorname{Spec}(\mathbb{Z})} C$ in $\bar{U}$. Up to replacing $\bar{U}$ with an open subscheme, we may and we shall assume that the induced morphisms $\bar{U} \rightarrow \mathrm{X}_{\mathcal{H}, \Sigma}^{\text {tor }}$ and $\bar{U} \rightarrow E(\sigma) \times_{\mathrm{Spec}(\mathbb{Z})} C$ have connected geometric fibers. Let $\varphi: U \rightarrow \mathbf{Z}$ 
denote the induced morphism. Then we have the following two cases for $Y \in$ $\operatorname{Supp}\left(R \Psi_{\mathrm{X}_{\mathcal{H}}}(\Lambda[d])\right)$ :

(1) The pullback of $Y$ to $U_{\bar{s}}$ is empty.

(2) The pullback $Y_{U}$ of $Y$ to $U_{\bar{s}}$ coincides with the pullback under $\varphi$ of some $Y_{Z}^{\natural} \in \operatorname{Supp}\left(R \Psi_{Z}\left(\Lambda\left[d_{Z}\right]\right)\right)$, where $d_{Z}:=\operatorname{dim}\left(Z_{\eta}\right)$. Moreover, the pullbacks of sheaves $\mathcal{G}$ in $\operatorname{Loc}_{Y}\left(R \Psi_{\mathrm{X}_{\mathcal{H}}}(\Lambda[d])\right)$ and $\mathcal{G}_{Z}^{\natural}$ in $\operatorname{Loc}_{Y_{Z}^{\natural}}\left(R \Psi_{Z}\left(\Lambda\left[d_{Z}\right]\right)\right)$ to $Y_{U}$ define a bijection

$$
\operatorname{Loc}_{Y}\left(R \Psi_{\mathrm{X}_{\mathcal{H}}}(\Lambda[d])\right) \cong \operatorname{Loc}_{Y_{\mathrm{Z}}^{\natural}}\left(R \Psi_{\mathrm{Z}}\left(\Lambda\left[d_{\mathrm{Z}}\right]\right)\right),
$$

such that, when $\mathcal{G}$ is matched with $\mathcal{G}_{Z}^{\natural}$, we have

$$
m(Y, \mathcal{G})=m\left(Y_{\mathrm{Z}}^{\natural}, \mathcal{G}_{\mathrm{Z}}^{\natural}\right) .
$$

Proof. Since $\varphi: U \rightarrow \mathrm{Z}$ is smooth, we have

$$
\left.\varphi^{*} R \Psi_{\mathrm{Z}}(\Lambda) \cong R \Psi_{U}(\Lambda) \cong\left(R \Psi_{\mathrm{X}_{\mathcal{H}}}(\Lambda)\right)\right|_{U_{\bar{s}}}
$$

(see [3, XV, 2.1] and [16, XIII, 2.1.5]). Moreover, since $\varphi$ is smooth of relative dimension $d^{\mathrm{Z}}:=d-d_{\mathrm{Z}}$, the functor $\varphi^{*}\left[d^{\mathrm{Z}}\right]$ is $t$-exact for the middle perversity by $[6,4.2 .5]$. Therefore, it sends a Jordan-Hölder filtration to a filtration. By [6, 4.2.6.2], since $\varphi: U \rightarrow \varphi(U)$ has nonempty connected geometric fibers, $\varphi^{*}\left[d^{\mathrm{z}}\right]$ induces a fully faithful embedding of the category of perverse sheaves over $\varphi(U)$ as a thick subcategory of the category of perverse sheaves over $U$. Consequently, the restriction of the functor $\varphi^{*}\left[d^{\mathrm{Z}}\right]$ to the category of perverse sheaves over $\varphi(U)$ respects Jordan-Hölder filtrations, as desired. (For our purpose, the restriction from $Z$ to its open subscheme $\varphi(U)$ is irrelevant because any $Y_{Z}^{\natural}$ in case (2) has a nonempty intersection with the open subscheme $\varphi(U)_{\bar{s}}$ of $Z_{\bar{s}}$.)

Lemma 3.7.9 shows that studying the nearby cycles $R \Psi_{\mathrm{X}_{\mathcal{H}}}(\Lambda)$ near the boundary of any $\left(\mathbf{X}_{\mathcal{H}, \Sigma}^{\text {tor }}\right)_{\bar{s}}$ is essentially the same as studying the nearby cycles $R \Psi_{Z}(\Lambda)$ over the smaller analogues $Z_{\bar{s}}$ of $\left(\mathrm{X}_{\mathcal{H}}\right)_{\bar{s}}$. More precisely, we have the following:

Proposition 3.7.13. Suppose that Assumption 3.7.7 holds. Then every $Y$ in $\operatorname{Supp}\left(R \Psi_{\mathrm{X}_{\mathcal{H}}}(\Lambda[d])\right)$ is a (reduced) well-positioned subscheme of $\left(\mathrm{X}_{\mathcal{H}}\right)_{\bar{s}}$ as in Definition 2.2.1 (with $\mathrm{T}=\bar{s}$ there), with minimal and toroidal compactifications $Y^{\mathrm{min}}$ and $Y_{\Sigma}^{\text {tor }}$ as in Definition 2.3.1 and Theorem 2.3.2.

Under the assumption (in (4) of Proposition 2.1.2) that $\Sigma$ is smooth, we have

$$
\operatorname{Supp}\left(R \Psi_{\mathrm{X}_{\mathcal{H}, \Sigma}^{\text {tor }}}(\Lambda[d])\right)=\left\{Y_{\Sigma}^{\text {tor }}: Y \in \operatorname{Supp}\left(R \Psi_{\mathrm{X}_{\mathcal{H}}}(\Lambda[d])\right)\right\}
$$


For each $Y \in \operatorname{Supp}\left(R \Psi_{\mathrm{X}_{\mathcal{H}}}(\Lambda[d])\right)$ and each $\mathcal{G} \in \operatorname{Loc}_{Y}\left(R \Psi_{\mathrm{X}_{\mathcal{H}}}(\Lambda[d])\right)$, we have a canonical extension $\mathcal{G}_{\Sigma}^{\text {tor }} \in \operatorname{Loc}_{Y_{\Sigma}^{\text {tor }}}\left(\operatorname{Supp}\left(R \Psi_{\mathrm{X}_{\mathcal{H}, \Sigma}^{\text {tor }}}(\Lambda[d])\right)\right)$, with

$$
m(Y, \mathcal{G})=m\left(Y_{\Sigma}^{\text {tor }}, \mathcal{G}_{\Sigma}^{\text {tor }}\right) .
$$

Proof. Let $Y^{\mathrm{min}}$ and $Y_{\Sigma}^{\text {tor }}$ denote the (reduced) schematic closures of $Y$ in $\left(\mathrm{X}_{\mathcal{H}}^{\min }\right)_{\bar{s}}$ and $\left(\mathrm{X}_{\mathcal{H}, \Sigma}^{\text {tor }}\right)_{\bar{s}}$, respectively. Suppose $\bar{U}, U$, and $\varphi: U \rightarrow Z$ are as in Corollary 2.1.7 and Lemma 3.7.9. Then Lemma 3.7.9, with the $Y_{Z}^{\natural}$ there, implies that $\varphi(U)_{\bar{s}} \cap$ $Y_{Z}^{\natural}=\varphi(U)_{\bar{s}} \cap Y^{\min }$ as subsets of $Z_{\bar{s}}$, and that the pullback of the canonical open immersion $J_{Y_{\Sigma}^{\text {tor }}}: Y \rightarrow Y_{\Sigma}^{\text {tor }}$ (induced by the canonical open immersion $J_{X_{\mathcal{H}, \Sigma}^{\text {tor }}}$ : $\mathrm{X}_{\mathcal{H}} \hookrightarrow \mathrm{X}_{\mathcal{H}, \Sigma}^{\text {tor }}$ ) under $\bar{U} \rightarrow \mathrm{M}_{\mathcal{H}, \Sigma}^{\text {tor }}$ coincides with the pullback of the canonical open immersion

$$
\left.J_{Y_{\mathrm{Z}}^{\natural} \mathrm{Z}(E(\sigma) \times \operatorname{Spec}(\mathbb{Z})} C\right): Y_{\mathrm{Z}}^{\natural} \times_{\mathrm{Z}}\left(E \times_{\operatorname{Spec}(\mathbb{Z})} C\right) \hookrightarrow Y_{\mathrm{Z}}^{\natural} \times_{\mathrm{Z}}\left(E(\sigma) \times_{\operatorname{Spec}(\mathbb{Z})} C\right)
$$

induced by $J_{E(\sigma)}: E \hookrightarrow E(\sigma)$ and the identity morphisms on $Y_{Z}^{\natural}$ and $C$. Since the respective pullbacks of the étale morphisms $\bar{U} \rightarrow \mathrm{X}_{\mathcal{H}, \Sigma}^{\text {tor }}$ and $\bar{U} \rightarrow E(\sigma) \times_{\operatorname{Spec}(\mathbb{Z})} C$ to $Z_{[\sigma]}$ and $E_{\sigma} \times_{\operatorname{Spec}(\mathbb{Z})} C$ are both open immersions (see Corollary 2.1.7), by gluing in the Zariski topology, the pullback of the canonical isomorphism $\Xi_{\sigma} \cong Z_{[\sigma]}$ in (8) of Proposition 2.1.2 to $\bar{s}$ induces a canonical isomorphism $Y_{\mathrm{Z}}^{\natural} \times_{\mathrm{Z}} \Xi_{\sigma} \cong Y_{\mathrm{Z}_{[\sigma]}}$, where $Y_{\mathrm{Z}_{[\sigma]}}:=\mathrm{Z}_{[\sigma]} \times_{\mathrm{X}_{\mathcal{H}, \Sigma}^{\text {tor }}} Y_{\Sigma}^{\text {tor }}$. Consequently, the canonical isomorphism $\mathfrak{X}_{\sigma}^{\circ}=(\Xi(\sigma))_{\bigcup_{\tau \in \Sigma_{Z}^{+}, \bar{\tau} \subset \bar{\sigma}} \Xi_{\tau}} \cong\left(X_{\mathcal{H}, \Sigma}^{\text {tor }}\right)_{\bigcup_{\tau \in \Sigma_{Z}^{+}, \bar{\tau} \subset \bar{\sigma}} Z_{[\tau]}}$ in Proposition 2.1.3 induces a canonical isomorphism $\left(Y_{\mathrm{Z}}^{\natural} \times \mathrm{Z} \Xi(\sigma)\right)_{Y_{Z}^{\natural} \times \mathrm{Z} \Xi_{\sigma}}^{\wedge} \cong\left(Y_{\Sigma}^{\text {tor }}\right)_{Y_{Z_{[\sigma]}}}^{\wedge}$, and the analogues of the other properties in Proposition 2.1.2 follow. By matching also the closed subscheme $\bigcup_{\tau \in \Sigma_{Z}^{+}, \bar{\tau} \subset \bar{\sigma}}\left(Y_{Z}^{\natural} \times \mathrm{Z} \Xi_{\tau}\right)$ of $Y_{Z}^{\natural} \times \mathrm{Z} \Xi(\sigma)$ with the locally closed subscheme $\bigcup_{\tau \in \Sigma_{\mathrm{Z}}^{+}, \bar{\tau} \subset \bar{\sigma}} Y_{\mathrm{Z}_{[\tau]}}$ of $Y_{\Sigma}^{\text {tor }}$ (see Corollary 2.1.7 again), the analogue of Proposition 2.1.3 also follows. This shows that $Y$ is a well-positioned subscheme, and that $Y_{Z}^{\natural}$ and $Y_{Z}:=Z \times{ }_{x_{\mathcal{H}}^{\min }} Y^{\min }$ coincide as subsets of $Z_{\bar{s}}$, by Theorem 2.3.2.

As for the assertions in the last paragraph, under the assumption that $\Sigma$ is smooth, the morphism $E(\sigma) \rightarrow \operatorname{Spec}(\mathbb{Z})$ is smooth for each $\sigma$ in $\Sigma_{Z}$. Hence, the same argument as in the proof of Lemma 3.7 .9 (see, in particular, (3.7.12)) shows that $R \Psi_{\mathrm{X}_{\mathcal{H}}^{\text {tor }}}(\Lambda[d])$ is étale locally near $\left(Z_{[\sigma]}\right)_{\bar{s}}$ the pullback of $R \Psi_{Z}(\Lambda[d])$, and so its supports are étale locally the pullbacks of those of $R \Psi_{Z}\left(\Lambda\left[d_{Z}\right]\right)$. Thus, we can conclude the proof by comparing these with the assertions in Lemma 3.7.9 for $\operatorname{Supp}\left(R \Psi_{\mathrm{X}_{\mathcal{H}}}(\Lambda[d])\right)$.

REMARK 3.7.16. It may happen that a support $Y$ does not meet any boundary strata in the sense that $Y_{\mathrm{Z}}=\mathrm{Z} \times_{\mathrm{X}_{\mathcal{H}}^{\min }} Y^{\min }$ is empty for each stratum $\mathrm{Z}$ of $\mathrm{X}_{\mathcal{H}}^{\min }$. Equivalently, by Proposition 3.7.13, $Y_{\mathrm{Z}_{[\sigma]}}=\mathrm{Z}_{[\sigma]} \times_{\mathrm{X}_{\mathcal{H}, \Sigma}^{\text {tor }}} Y_{\Sigma}^{\text {tor }}$ is empty for each 
stratum $\mathrm{Z}_{[\sigma]}$ of $\mathrm{X}_{\mathcal{H}, \Sigma}^{\text {tor }}$. In this case, we have $Y=Y^{\text {min }}$ and $Y=Y_{\Sigma}^{\text {tor }}$. (For studying supercuspidal representations of $\mathrm{G}\left(\mathbb{Q}_{p}\right)$, such supports might be the most interesting.)

\section{Well-positioned étale sheaves}

4.1. Definition. Let $\mathrm{T}$ be a locally noetherian scheme over $\mathrm{S}$ as in Section 2.2, which we assume to be separated and of finite type over a regular scheme of dimension $\leqslant 1$, or over a quasiexcellent finite-dimensional scheme. Consider any rational prime number $\ell \neq p$. Let $\Lambda$ be a coefficient ring that is either $\mathbb{Z} / \ell^{m} \mathbb{Z}$ (for some integer $m \geqslant 1$ ), $\mathbb{Z}_{\ell}, \mathbb{Q}_{\ell}, \overline{\mathbb{Q}}_{\ell}$, or a finite extension of any of these. For simplicity, we shall also denote by $\Lambda$ the constant étale sheaf with values in $\Lambda$. We shall denote by $D_{c}^{b}(\cdot, \Lambda)$ the bounded derived category of $\Lambda$-étale constructible sheaves over $(\cdot)$, when defined. (See [15, 1.1], [17], and [49] when $\Lambda$ is not torsion.) When discussing perverse sheaves, we shall assume (as in $[6,2.2 .14]$ ) that $\mathrm{T}=\operatorname{Spec}(k)$ for some field $k$ that is either algebraically closed or finite, and that $\Lambda=\mathbb{Q}_{\ell}$ or $\overline{\mathbb{Q}}_{\ell}$.

Given any stratum $Z$ of $X_{\mathcal{H}}^{\min }$, we shall write

$$
\tilde{Z}:=\bigcup_{[\tau] \in \Sigma_{\mathrm{Z}}^{+} / \Gamma} \mathrm{Z}_{[\tau]},
$$

which is the reduced subscheme of the preimage of $Z$ in $X_{\mathcal{H}, \Sigma}^{\text {tor }}$. Suppose $Y$ is a wellpositioned subset of $\left(X_{\mathcal{H}}\right)_{T}$, with associated $Y^{\natural}=\left\{Y_{Z}^{\natural}\right\}_{Z}$ as in Definition 2.2.1. Let us equip $Y$ and $Y_{Z}^{\natural}$ with their canonical reduced subscheme structures, for all $Z$. Let $Y^{\text {min }}, Y_{\Sigma}^{\text {tor }}$, and $Y_{Z}=Z \times_{X_{\mathcal{H}}^{\min }} Y^{\text {min }}$ be as in Definition 2.3.1 and Theorem 2.3.2. Let

$$
\mathrm{Y}_{\tilde{Z}}:=\tilde{Z} \times_{\mathrm{X}_{\mathcal{H}, \Sigma}^{\text {tor }}} \mathrm{Y}_{\Sigma}^{\text {tor }} \text {. }
$$

Let

$$
a_{\mathrm{Y}_{\tilde{Z}}}: \mathrm{Y}_{\tilde{Z}} \rightarrow \mathrm{Y}_{\Sigma}^{\text {tor }}
$$

and

$$
a_{Y_{\tilde{Z}}}^{\natural}: Y_{\tilde{Z}} \rightarrow Y_{Z}^{\natural}
$$

denote the canonical morphisms.

DEFinition 4.1.1. Consider a collection $\mathcal{U}_{\mathrm{Z}}=\left\{\left(\bar{U}_{i}, a_{\bar{U}_{i}}, a_{\bar{U}_{i}, C}^{\natural}\right)\right\}_{i \in I_{Z}}$, where

$$
a_{\bar{U}_{i}}: \bar{U}_{i} \rightarrow \mathrm{X}_{\mathcal{H}, \Sigma}^{\mathrm{tor}}
$$

and

$$
a_{\bar{U}_{i}, E\left(\sigma_{i}\right)}^{\natural}: \bar{U}_{i} \rightarrow E\left(\sigma_{i}\right) \times \operatorname{Spec}(\mathbb{Z}) C
$$


are étale morphisms like the $\bar{U} \rightarrow \mathrm{X}_{\mathcal{H}, \Sigma}^{\text {tor }}$ and $\bar{U} \rightarrow E(\sigma) \times_{\operatorname{Spec}(\mathbb{Z})} C$ in Corollary 2.1.7, which we assume to have connected geometric fibers (which is possible up to replacing $\bar{U}$ with an open subscheme).

(1) We say that $\mathcal{U}_{Z}$ induces an affine open covering of $\tilde{Z}$ if each $\bar{U}_{i}$ is affine and if

$$
\left\{\bar{U}_{i}^{+}:=\bar{U}_{i} \times_{X_{\mathcal{H}, \Sigma}^{\text {tor }}} \tilde{Z} \hookrightarrow \tilde{Z}\right\}_{i \in I_{Z}}
$$

is an affine open covering of $\tilde{Z}$.

(2) We say that $\mathcal{U}_{Z}$ is adapted to a well-positioned subset $Y$ (as above) if the pullback of $Y_{\Sigma}^{\text {tor }}$ under $a_{\bar{U}_{i}}$ coincides with the pullback of $Y_{Z}^{\natural}$ under the morphism

$$
a_{\bar{U}_{i}}^{\natural}: \bar{U}_{i} \rightarrow \mathrm{Z}
$$

induced by $a_{\bar{U}_{i}, E\left(\sigma_{i}\right)}^{\natural}$, for each $Z$ and for each $i \in I_{Z}$.

LEMMA 4.1.2. Given any $\mathcal{U}_{Z}$ inducing an affine open covering of $\tilde{Z}$ as in Definition 4.1.1, up to replacing $\mathcal{U}_{\mathrm{Z}}$ with an étale refinement, we may assume that $\mathcal{U}_{\mathrm{Z}}$ is adapted to any finite collection $\left\{\mathrm{Y}_{i}\right\}_{i \in I}$ of well-positioned subsets of $\left(\mathrm{X}_{\mathcal{H}}\right)_{\mathrm{T}}$.

Proof. This is achieved by an analogue of the argument of the proof of (7) of Theorem 2.3.2, by refining the output of Corollary 2.1.7, using Artin's approximation, which matches not just the stratifications but also the pullbacks of the locally closed subschemes $Y_{i}$ and $Y_{i, \Xi(\sigma)}^{\natural}$ of $\left(X_{\mathcal{H}}\right)_{\top}$ and $(\Xi(\sigma))_{\mathrm{T}}$, respectively, for all $i$ (assuming without loss of generality that $S$ is excellent and that $T$ is of finite type over $\mathrm{S})$.

Definition 4.1.3. With the setting (of $\mathrm{Y}, \mathrm{Y}_{\Sigma}^{\text {tor }}$, and so forth) as above, we say that a complex $\mathcal{F}$ in $D_{c}^{b}\left(\mathrm{Y}_{\Sigma}^{\text {tor }}, \Lambda\right)$ is a well-positioned complex (over $\mathrm{Y}_{\Sigma}^{\text {tor }}$ ) if there exists a collection

$$
\mathcal{F}^{\natural}=\left\{\left(\mathcal{F}_{Z}^{\natural}, \iota_{Z}\right)\right\}_{Z}
$$

indexed by the strata $\mathrm{Z}$ of $\mathrm{X}_{\mathcal{H}}^{\min }$, where $\mathcal{F}_{Z}^{\natural} \in D_{c}^{b}\left(Y_{Z}^{\natural}, \Lambda\right)$, and where

$$
\iota: a_{Y_{\tilde{Z}}}^{*} \mathcal{F} \stackrel{\sim}{\rightarrow} a_{Y_{\tilde{Z}}^{\natural}}^{\natural, *} \mathcal{F}_{Z}^{\natural}
$$

is an isomorphism in $D_{c}^{b}\left(\mathrm{Y}_{\tilde{\mathrm{Z}}}, \Lambda\right)$, for each $\mathrm{Z}$, satisfying the following compatibility condition: For each $Z$, there exists some collection

$$
\mathcal{U}_{\mathrm{Z}}=\left\{\left(\bar{U}_{i}, a_{\bar{U}_{i}}, a_{\bar{U}_{i}, E\left(\sigma_{i}\right)}^{\natural}\right)\right\}_{i \in I_{\mathrm{Z}}}
$$


which induces an affine open covering of $\tilde{Z}$ and is adapted to $Y$ as in Definition 4.1.1, which induces canonical morphisms

$$
a_{\mathrm{Y}_{\bar{U}_{i}}}: \mathrm{Y}_{\bar{U}_{i}}:=\bar{U}_{i} \times_{\mathrm{X}_{\mathcal{H}, \Sigma}^{\text {tor }}} \mathrm{Y}_{\Sigma}^{\text {tor }} \rightarrow \mathrm{Y}_{\Sigma}^{\text {tor }}
$$

and

$$
a_{\mathrm{Y}_{\bar{U}_{i}}}^{\natural}: \mathrm{Y}_{\bar{U}_{i}} \rightarrow \mathrm{Y}_{\mathrm{Z}}^{\natural},
$$

such that the restriction of $\iota_{z}$ to

$$
\mathrm{Y}_{\bar{U}_{i}^{+}}:=\mathrm{Y}_{\bar{U}_{i}} \times_{\mathrm{X}_{\mathcal{H}, \Sigma}^{\mathrm{tor}}} \tilde{\mathrm{Z}}
$$

for each $i \in I_{Z}$, extends to an isomorphism

$$
\iota_{\bar{U}_{i}}: a_{\mathrm{Y}_{\bar{U}_{i}}}^{*} \mathcal{F} \stackrel{\sim}{\rightarrow} a_{\mathrm{Y}_{\bar{U}_{i}}^{\natural, *}}^{\natural} \mathcal{F}_{\mathrm{Z}}^{\natural}
$$

in $D_{c}^{b}\left(\mathrm{Y}_{\bar{U}_{i}}, \Lambda\right)$.

We say that an $\mathcal{F}$ as above is a well-positioned sheaf (respectively wellpositioned perverse sheaf, when $\mathrm{T}$ and $\Lambda$ are as in the beginning of this section) if, moreover, $\mathcal{F}$ is a sheaf (respectively perverse sheaf). We often suppress the isomorphisms $\iota$ from the notation when they are canonical ones or are clear from the context.

REMARK 4.1.6. Certainly, the above applies to the special case with $Y=\left(X_{\mathcal{H}}\right)_{\top}$ and $Y^{\natural}=\left\{Z_{T}\right\}_{Z}$, as in Section 3.1.

REMARK 4.1.7. In Definition 4.1.3, if we replace $\mathcal{U}_{\mathrm{Z}}=\left\{\left(\bar{U}_{i}, a_{\bar{U}_{i}}, a_{\bar{U}_{i}, E\left(\sigma_{i}\right)}^{\natural}\right)\right\}_{i \in I_{Z}}$ with a refinement $\mathcal{U}_{Z}^{\prime}=\left\{\left(\bar{U}_{i^{\prime}}^{\prime}, a_{\bar{U}_{i^{\prime}}^{\prime}}, a_{\bar{U}_{i^{\prime}}^{\prime}, E\left(\sigma_{i^{\prime}}\right)}^{\natural}\right)\right\}_{i^{\prime} \in I_{Z}^{\prime}}$, then the isomorphism $\iota_{\bar{U}_{i}}$ in (4.1.5) induces an isomorphism $\iota_{\bar{U}_{i^{\prime}}^{\prime}}$ in $D_{c}^{b}\left(\mathrm{Y}_{\bar{U}_{i^{\prime}}^{\prime}}, \Lambda\right)$ when $\bar{U}_{i^{\prime}}^{\prime}$ refines $\bar{U}_{i}$.

REMARK 4.1.8. For a well-positioned perverse sheaf $\mathcal{F}$ as in Definition 4.1.3, when $Y_{C}^{\natural} \rightarrow Y_{Z}^{\natural}$ is smooth, we typically have $\mathcal{F}_{Z}^{\natural}$ perverse only up to shifting by $\operatorname{dim}(Y)-\operatorname{dim}\left(Y_{Z}^{\natural}\right)$. (See Lemma 4.2.13 below.)

REMARK 4.1.9. In Definition 4.1.3, we allow $\mathcal{F}_{Z}^{\natural}=0$, which will be the case for any $Z$ such that $\mathcal{F}=0$ near $Y_{\tilde{Z}}$. For $Z \neq Z^{\prime}$, we do not require any compatibility between $\left(\mathcal{F}_{Z}^{\natural}, \iota_{Z}\right)$ and $\left(\mathcal{F}_{Z^{\prime}}^{\natural}, \iota_{Z^{\prime}}\right)$.

REMARK 4.1.10. In Definition 4.1.3, the complex $\mathcal{F}$ does not necessarily determine the collection $\mathcal{F}^{\natural}$. Nevertheless, under the assumption (in (4) of Proposition 2.1.2) that $\Sigma$ is smooth, if $Y_{C}^{\natural} \rightarrow Y_{Z}^{\natural}$ is smooth and has nonempty 
connected geometric fibers, for each $Z$, and if $\mathcal{F}$ and $\mathcal{F}_{Z}^{\natural}$ are either lisse sheaves or perverse sheaves, then $\mathcal{F}_{Z}^{\natural}$ is uniquely determined by $\mathcal{F}$, by [6, 4.2.6.2]. Alternatively, if $\Lambda=\overline{\mathbb{Q}}_{\ell}$, and if Assumption 4.3.1 below holds, then $\mathcal{F}_{Z}^{\natural}$ is uniquely determined by $\mathcal{F}$ (by Theorem 4.3.16; see Remark 4.3.32 below).

REMARK 4.1.11. The automorphic étale sheaves $\mathcal{V}_{\xi}$ defined as in [45, Section 3] typically (for nontrivial $\xi$ ) do not extend to well-positioned complexes over $\mathrm{X}_{\mathcal{H}, \Sigma}^{\text {tor }}$.

As a partial justification of Definition 4.1.3, we have the following:

LEMMA 4.1.12. In each of the contexts of Propositions 2.4.1, 2.4.2, and 2.4.3 (where $\mathcal{H}^{\prime}=\mathcal{H}$ and $\Sigma^{\prime}=\Sigma$ in the context of Proposition 2.4.1), suppose $\mathcal{F}$ is a well-positioned complex over $\mathrm{Y}_{\Sigma}^{\text {tor }}$, equipped with a collection $\mathcal{F}^{\natural}=\left\{\left(\mathcal{F}_{Z}^{\natural}, \iota_{Z}\right)\right\}_{\mathrm{Z}}$, as in Definition 4.1.3. Let $\mathcal{F}^{\prime}$ denote the pullback of $\mathcal{F}$ under $\mathrm{Y}_{\Sigma^{\prime}}^{\prime \text { tor }} \rightarrow \mathrm{Y}_{\Sigma}^{\text {tor }}$. For each stratum $Z^{\prime}$ of $\mathbf{X}_{\mathcal{H}^{\prime}}^{\min }$ above a stratum $\mathbf{Z}$ of $\mathbf{X}_{\mathcal{H}}^{\min }$, let $\mathcal{F}_{Z^{\prime}}^{\prime, t}$ denote the pullback of $\mathcal{F}_{Z}^{\natural}$ under $Y_{Z^{\prime}}^{\prime, \natural} \rightarrow Y_{Z}^{\natural}$, and let $\iota_{Z^{\prime}}^{\prime}$ denote the pullback of $\iota_{Z}$ under $Y_{\tilde{Z}}^{\prime} \rightarrow Y_{\tilde{Z}}$, where $\mathrm{Y}_{\tilde{Z}}^{\prime}$ denotes the preimage of $\tilde{Z}^{\prime}$ in $\mathrm{Y}_{\Sigma^{\prime}}^{\prime \text { tor }}$. Then $\mathcal{F}^{\prime}$ is a well-positioned complex over $Y_{\Sigma^{\prime}}^{\prime \text {,tor }}$, equipped with the collection $\mathcal{F}^{\prime, \natural}:=\left\{\left(\mathcal{F}_{Z^{\prime}}^{\prime, \natural}, \iota_{Z^{\prime}}^{\prime}\right)\right\}_{Z^{\prime}}$.

If $\mathcal{F}$ is a well-positioned sheaf, so is $\mathcal{F}^{\prime}$. Under the assumption (in (4) of Proposition 2.1.2) that $\Sigma$ and $\Sigma^{\prime}$ are smooth, if $\mathcal{F}$ is a well-positioned perverse sheaf; if $Y_{C}^{\natural} \rightarrow Y_{Z}^{\natural}$ and the induced morphism $Y_{C^{\prime}}^{\prime, \natural} \rightarrow Y_{Z}^{\natural}$ are smooth of the same relative dimension $d^{Z}-d_{E}$, where $d_{E}$ is the relative dimension of $E$ over $\operatorname{Spec}(\mathbb{Z})$, for each $\mathbf{Z}^{\prime}$ above $\mathrm{Z}$; and if $\mathcal{F}_{Z}^{\natural}\left[-d^{Z}\right]$ is a perverse sheaf, for each $\mathrm{Z}$; then $\mathcal{F}^{\prime}$ is also a well-positioned perverse sheaf.

Proof. For each stratum $Z$ of $X_{\mathcal{H}}^{\min }$, let $\mathcal{U}_{Z}=\left\{\left(\bar{U}_{i}, a_{\bar{U}_{i}}, a_{\bar{U}_{i}, E\left(\sigma_{i}\right)}^{\natural}\right)\right\}_{i \in I_{Z}}$ be as in Definition 4.1.3. Suppose $Z^{\prime}$ is a stratum of $X_{\mathcal{H}^{\prime}}^{\min }$ above $Z$, with $\tilde{Z}^{\prime}$ the reduced subscheme of its preimage in $X_{\mathcal{H}^{\prime}, \Sigma^{\prime}}^{\text {tor }}$. Suppose there exists a collection

$$
\mathcal{U}_{Z^{\prime}}^{\prime}=\left\{\left(\bar{U}_{i^{\prime}}, a_{\bar{U}_{i^{\prime}}}, a_{\bar{U}_{i^{\prime}}, E^{\prime}\left(\sigma_{i^{\prime}}\right)}^{\natural}\right)\right\}_{i^{\prime} \in I_{Z^{\prime}}}
$$

for $X_{\mathcal{H}^{\prime}, \Sigma^{\prime}}^{\text {tor }}$ such that it induces an affine open covering of $\tilde{Z}^{\prime}$ and is adapted to $Y^{\prime}$ as in Definition 4.1.1, and such that it refines the pullback of $\mathcal{U}_{\mathrm{Z}}$ in the sense that, for each $i^{\prime} \in I_{Z^{\prime}}$, there exists some $i \in I_{Z}$ such that $a_{\bar{U}_{i^{\prime}}}$ and $a_{\bar{U}_{i^{\prime}}, E\left(\sigma_{i^{\prime}}\right)}^{\natural}$ lift the pullbacks of $a_{\bar{U}_{i}}$ and $a_{\bar{U}_{i}, E\left(\sigma_{i}\right)}^{\natural}$, respectively, via some étale morphisms $\bar{U}_{i^{\prime}} \rightarrow$ $\bar{U}_{i} \times \chi_{\mathcal{H}, \Sigma}^{\text {tor }} X_{\mathcal{H}^{\prime}, \Sigma^{\prime}}^{\text {tor }}$ and $\bar{U}_{i^{\prime}} \rightarrow \bar{U}_{i} \times \Xi\left(\sigma_{i}\right) \Xi^{\prime}\left(\sigma_{i}^{\prime}\right)$. Then the pullbacks of $\iota \mathrm{Z}$ and $\iota_{\bar{U}_{i}}$ to $\tilde{Z}^{\prime}:=\tilde{Z} \times Y_{\Sigma}^{\text {tor }} Y_{\Sigma^{\prime}}^{\prime \text {,tor }}$ and $Y_{\bar{U}_{i^{\prime}}}^{\prime}:=\bar{U}_{i^{\prime}} \times_{\mathcal{H}_{\mathcal{H}^{\prime}, \Sigma^{\prime}}^{\text {tor }}} \mathrm{Y}_{\Sigma^{\prime}}^{\prime \text {,tor }}$, respectively, induce the desired $\iota_{Z^{\prime}}^{\prime}$ and $\iota_{\bar{U}_{i^{\prime}}}^{\prime}$. The question (in the last paragraph of the lemma) of whether $\mathcal{F}^{\prime}$ is a sheaf 
or a perverse sheaf can be answered étale locally, essentially by definition. (For perverse sheaves, see [6, 2.2.12 and 2.2.19].) Hence, by using the isomorphisms $\iota_{\bar{U}^{\prime}}^{\prime}$, and (for perverse sheaves) by the $t$-exactness of smooth morphisms up to shifting by the relative dimensions (see [6, 4.2.5]), the question has an affirmative answer by the assumptions we made. It remains to construct such a $\mathcal{U}_{Z^{\prime}}^{\prime}$.

In the context of Proposition 2.4.1, we can just take $\mathcal{U}_{\mathrm{Z}^{\prime}}^{\prime}=\mathcal{U}_{\mathrm{Z}}$, since these collections are defined over the same $X_{\mathcal{H}, \Sigma}^{\text {tor }}$ regardless of the base change $\mathrm{T}^{\prime} \rightarrow \mathrm{T}$.

In each of the contexts of Propositions 2.4.2 and 2.4.3, let us fixed a choice of some $i \in I_{Z}$. Let $\bar{U}_{i}^{+}$denote the pullback of $\tilde{Z}$ under $a_{\bar{U}_{i}}: \bar{U}_{i} \rightarrow \mathrm{X}_{\mathcal{H}, \Sigma}^{\text {tor }}$. Suppose that $x$ is a point of $X_{\mathcal{H}^{\prime}, \Sigma^{\prime}}^{\text {tor }}$ whose image $y$ in $X_{\mathcal{H}, \Sigma}^{\text {tor }}$ is contained in the image of the open immersion $\bar{U}_{i}^{+} \rightarrow \tilde{Z}$ induced by $a_{\bar{U}_{i}}$, so that $y$ lies on the stratum $\mathrm{Z}_{\left[\sigma_{i}\right]}$ of $X_{\mathcal{H}, \Sigma}^{\text {tor }}$. Up to replacing $\bar{U}_{i}$ with an affine open subscheme still containing $y$, we may and we shall assume that $a_{\bar{U}_{i}, E\left(\sigma_{i}\right)}^{\natural}: \bar{U}_{i} \rightarrow E\left(\sigma_{i}\right) \times_{\operatorname{Spec}(\mathbb{Z})} C$ is induced by an étale morphism $a_{\bar{U}_{i}, \Xi\left(\sigma_{i}\right)}^{\natural}: \bar{U}_{i} \rightarrow \Xi\left(\sigma_{i}\right)$ such that, over the image of this étale morphism, $\Xi\left(\sigma_{i}\right)$ is isomorphic to $E\left(\sigma_{i}\right) \times \operatorname{Spec}(\mathbb{Z}) C$ as schemes over $C$ (cf. [45, Lemma 2.3]), and such that $a_{\bar{U}_{i}, E\left(\sigma_{i}\right)}^{\natural}$ and $a_{\bar{U}_{i}, \Xi\left(\sigma_{i}\right)}^{\natural}$ differ by this last isomorphism. Then $\bar{U}_{i}^{+}$coincides with the pullback of

$$
\Xi\left(\sigma_{i}\right)^{+}:=\bigcup_{\tau \in \Sigma_{\mathrm{Z}}^{+}, \bar{\tau} \subset \bar{\sigma}_{i}} \Xi_{\tau} \subset \Xi\left(\sigma_{i}\right)
$$

under $a_{\bar{U}_{i}, \Xi\left(\sigma_{i}\right)}^{\natural}$. Consider the pullbacks

$$
a_{\bar{U}_{i}^{\prime}}: \bar{U}_{i}^{\prime}:=\bar{U}_{i} \times \mathrm{X}_{\mathcal{H}, \Sigma}^{\mathrm{tor}} \mathrm{X}_{\mathcal{H}^{\prime}, \Sigma^{\prime}}^{\mathrm{tor}} \rightarrow \mathrm{X}_{\mathcal{H}^{\prime}, \Sigma^{\prime}}^{\mathrm{tor}}
$$

and

$$
a_{\bar{U}_{i}^{\prime \prime}, \Xi^{\prime}\left(\sigma_{i}^{\prime}\right)}^{\natural}: \bar{U}_{i}^{\prime \prime}:=\bar{U}_{i} \times_{\Xi\left(\sigma_{i}\right)} \Xi^{\prime}\left(\sigma_{i}^{\prime}\right) \rightarrow \Xi^{\prime}\left(\sigma_{i}^{\prime}\right)
$$

of $a_{\bar{U}_{i}}$ and $a_{\bar{U}_{i}, \Xi\left(\sigma_{i}\right)}^{\natural}$, respectively, where $\sigma_{i}^{\prime} \in \Sigma_{Z^{\prime}}^{\prime,+}$ is some cone such that $x$ lies on stratum $Z_{\left[\sigma_{i}^{\prime}\right]}$ of $\mathrm{X}_{\mathcal{H}^{\prime}, \Sigma^{\prime}}^{\text {tor }}$ Let $\bar{U}_{i}^{\prime}:=\bar{U}_{i} \times_{\mathrm{X}_{\mathcal{H}, \Sigma}^{\text {tor }}} \mathrm{X}_{\mathcal{H}^{\prime}, \Sigma^{\prime}}^{\text {tor }}, \bar{U}_{i}^{\prime,+}:=\bar{U}_{i}^{+} \times_{\mathrm{X}_{\mathcal{H}, \Sigma}^{\text {tor }}} \mathrm{X}_{\mathcal{H}^{\prime}, \Sigma^{\prime}}^{\text {tor }}$, $\bar{U}_{i}^{\prime \prime}:=\bar{U}_{i} \times_{\Xi\left(\sigma_{i}\right)} \Xi^{\prime}\left(\sigma_{i}^{\prime}\right)$, and $\bar{U}_{i}^{\prime \prime \prime}:=\bar{U}_{i}^{+} \times_{\Xi\left(\sigma_{i}\right)} \Xi^{\prime}\left(\sigma_{i}^{\prime}\right)$. Then $\bar{U}_{i}^{\prime \prime+}$ coincides with the pullback of $\tilde{Z}^{\prime}$, and $\bar{U}_{i}^{\prime \prime}++$ coincides with the pullback of

$$
\Xi^{\prime}\left(\sigma_{i}^{\prime}\right)^{+}:=\bigcup_{\tau^{\prime} \in \Sigma_{Z^{\prime}}^{\prime+}, \bar{\tau}^{\prime} \subset \bar{\sigma}_{i}^{\prime}} \Xi_{\tau^{\prime}}^{\prime} \subset \Xi^{\prime}\left(\sigma_{i}^{\prime}\right),
$$

and the induced morphisms $\bar{U}_{i}^{\prime,+} \rightarrow \tilde{Z}^{\prime}$ and $\bar{U}_{i}^{\prime \prime,+} \rightarrow \Xi^{\prime}\left(\sigma_{i}^{\prime}\right)^{+}$are open immersions with the same image, the preimage of $\bar{U}_{i}^{+}$in $\tilde{Z}^{\prime}$. Therefore, $\left(\bar{U}_{i}^{\prime}\right)_{\bar{U}_{i}^{\prime+}}^{\wedge}$ and $\left(\bar{U}_{i}^{\prime \prime}\right)_{\bar{U}_{i}^{\prime \prime}+}^{\wedge}$ 
are both canonically isomorphic to $\left(\mathrm{X}_{\mathcal{H}^{\prime}, \Sigma^{\prime}}^{\text {tor }}\right)_{\bar{U}_{i}^{\prime \prime}}^{\wedge}$. Moreover, for each affine open formal subscheme $\mathfrak{W}=\operatorname{Spf}(R)$ of $\left(\mathrm{X}_{\mathcal{H}^{\prime}, \Sigma^{\prime}}^{\text {tor }}\right)_{\bar{U}_{i}^{\prime},+}^{\wedge}$ the pullbacks to $W=\operatorname{Spec}(R)$ of the stratifications of $X_{\mathcal{H}^{\prime}, \Sigma^{\prime}}^{\text {tor }}$ and $\Xi^{\prime}\left(\sigma_{i}^{\prime}\right)$ coincide with each other.

By the same approximation argument as in the proofs of (7) of Theorem 2.3.2 and of Lemma 4.1.2, there exists a scheme $\bar{U}_{i}^{\prime \prime \prime}$ with étale morphisms $\bar{U}_{i}^{\prime \prime \prime} \rightarrow \bar{U}_{i}^{\prime}$ and $\bar{U}_{i}^{\prime \prime \prime} \rightarrow \bar{U}_{i}^{\prime \prime}$ such that the induced étale morphisms $a_{\bar{U}_{i}^{\prime \prime \prime}}: \bar{U}_{i}^{\prime \prime \prime} \rightarrow \mathrm{X}_{\mathcal{H}^{\prime}, \Sigma^{\prime}}^{\text {tor }}$ and $a_{\bar{U}_{i}^{\prime \prime \prime}, \Xi^{\prime}\left(\sigma_{i}^{\prime}\right)}^{\natural}: \bar{U}_{i}^{\prime \prime \prime} \rightarrow \Xi^{\prime}\left(\sigma_{i}^{\prime}\right)$ satisfy the same properties as the étale morphisms $\bar{U} \rightarrow$ $\mathrm{X}_{\mathcal{H}, \Sigma}^{\text {tor }}$ and $\bar{U} \rightarrow \Xi(\sigma)$ in (9) of Proposition 2.1.2 do, and are adapted to $\mathrm{Y}^{\prime}$ in the sense that the pullback of $Y_{\Sigma^{\prime}}^{\prime \text {,tor }}$ under $a_{\bar{U}_{i}^{\prime \prime \prime}}$ coincides with the pullback of $Y_{Z^{\prime}}^{\prime, \natural}$ under $a_{\bar{U}_{i}^{\prime \prime \prime}, \Xi^{\prime}\left(\sigma_{i}^{\prime}\right)}^{\natural}$; and such that the image of $a_{\bar{U}_{i}^{\prime \prime \prime}}$ contains $x$, so that $x$ is lifted to a point of $\bar{U}_{i}^{\prime \prime \prime}$. By [45, Lemma 2.3, and its proof], up to replacing $\bar{U}_{i}^{\prime \prime \prime}$ with an affine open subscheme still containing $x$, we may and we shall assume that, over the image of $a_{\bar{U}_{i}^{\prime \prime \prime}, \Xi^{\prime}\left(\sigma_{i}^{\prime}\right)}^{\natural}$, there is an isomorphism between $\Xi^{\prime}\left(\sigma_{i}^{\prime}\right)$ and $E^{\prime}\left(\sigma_{i}^{\prime}\right) \times_{\operatorname{Spec}(\mathbb{Z})} C^{\prime}$ as schemes over $C^{\prime}$, which lifts the pullback of the isomorphism between $\Xi\left(\sigma_{i}\right)$ and $E\left(\sigma_{i}\right) \times_{\operatorname{Spec}(\mathbb{Z})} C$ over the image of $a_{\bar{U}_{i}, \Xi^{\prime}\left(\sigma_{i}^{\prime}\right)}^{\natural}$, and hence $a_{\bar{U}_{i}^{\prime \prime \prime}, \Xi^{\prime}\left(\sigma_{i}^{\prime}\right)}^{\natural}$ induces an étale morphism $a_{\bar{U}_{i}^{\prime \prime}, E^{\prime}\left(\sigma_{i}^{\prime}\right)}^{\natural}: \bar{U}_{i}^{\prime \prime \prime} \rightarrow E^{\prime}\left(\sigma_{i}^{\prime}\right) \times \operatorname{Spec}(\mathbb{Z}) C^{\prime}$ lifting $a_{\bar{U}_{i}, E\left(\sigma_{i}\right)}^{\natural}$. Thus, since $i \in I_{Z}$ and $x$ are arbitrary, by collecting (and reindexing) such $\left(\bar{U}_{i}^{\prime \prime \prime}, a_{\bar{U}_{i}^{\prime \prime \prime}}, a_{\bar{U}_{i}^{\prime \prime \prime}, E^{\prime}\left(\sigma_{i}^{\prime}\right)}\right)$, we obtain the desired collection $\mathcal{U}_{Z^{\prime}}^{\prime}$ for $\mathrm{X}_{\mathcal{H}^{\prime}, \Sigma^{\prime}}^{\text {tor }}$

REMARK 4.1.13. In Lemma 4.1.12, without the assumptions in the last paragraph there, $\mathcal{F}^{\prime}$ is generally not a perverse sheaf even when $\mathcal{F}$ is.

4.2. General properties and examples. In this subsection, suppose $Y$ and $Y^{\prime}$ are well-positioned subsets of $\left(X_{\mathcal{H}}\right)_{T}$, such that $Y^{\prime}$ is a (locally closed) subset of $Y$, with associated $Y^{\natural}=\left\{Y_{Z}^{\natural}\right\}_{Z}$ and $Y^{\prime, \natural}=\left\{Y_{Z}^{\prime, \natural}\right\}_{Z}$, respectively, as in Definition 2.2.1. Let $Y_{\Sigma}^{\text {tor }}$ and $Y_{\Sigma}^{\prime \text {,tor }}$ denote the partial toroidal compactifications of $Y$ and $Y^{\prime}$, respectively, as in Definition 2.3.1. Let $J: Y_{\Sigma}^{\prime \text { tor }} \hookrightarrow Y_{\Sigma}^{\text {tor }}, J_{\tilde{Z}}: Y_{\tilde{Z}}^{\prime} \hookrightarrow Y_{\tilde{Z}}$, and $J_{Z}: Y_{Z}^{\prime, \natural} \hookrightarrow Y_{Z}^{\natural}$ denote the canonical locally closed immersions, for all $Z$. Let $a_{Y_{\tilde{Z}}}: Y_{\tilde{Z}} \rightarrow Y_{\Sigma}^{\text {tor }}, a_{Y_{\tilde{Z}}^{\prime}}: Y_{\tilde{Z}}^{\prime} \rightarrow Y_{\Sigma}^{\prime \prime \text { tor }}, a_{Y_{\tilde{Z}}}^{\natural}: Y_{\tilde{Z}} \rightarrow Y_{Z}^{\natural}$, and $a_{Y_{\tilde{Z}}^{\prime}}^{\natural}: Y_{\tilde{Z}}^{\prime} \rightarrow Y_{\tilde{Z}}^{\prime, \natural}$ denote the canonical morphisms, for each $Z$.

LEMMA 4.2.1. Suppose $\mathcal{F}$ and $\mathcal{F}^{\prime}$ are well-positioned complexes over $\mathrm{Y}_{\Sigma}^{\text {tor }}$ and $\mathrm{Y}_{\Sigma}^{\prime \text {,tor }}$, equipped with collections $\mathcal{F}^{\natural}=\left\{\left(\mathcal{F}_{\mathrm{Z}}^{\natural}, \iota_{\mathrm{Z}}\right)\right\}_{\mathrm{Z}}$ and $\mathcal{F}^{\prime, \natural}=\left\{\left(\mathcal{F}_{\mathrm{Z}}^{\prime, \natural}, \iota_{\mathrm{Z}}^{\prime}\right)\right\}_{\mathrm{Z}}$, respectively, as in Definition 4.1.3. Then: 
(1) $J_{!} \mathcal{F}^{\prime}$ is a well-positioned complex over $\mathrm{Y}_{\Sigma}^{\text {tor }}$, equipped with the collection $\left\{\left(J_{Z, !} \mathcal{F}_{Z}^{\prime, \natural}, J_{\tilde{Z}, !} \ell_{Z}^{\prime}\right)\right\}_{Z}$. In particular, $J_{!} \Lambda_{Y_{\Sigma}^{\prime} \text { tor }}$ is a well-positioned sheaf over $\mathrm{Y}_{\Sigma}^{\text {tor }}$, equipped with the collection $\left\{J_{\mathrm{Z}, !} \Lambda_{\mathrm{Y}_{\mathrm{Z}}^{\prime !}}\right\}_{\mathrm{Z}}$.

(2) $J^{*} \mathcal{F}$ is a well-positioned complex over $Y_{\Sigma}^{\prime \text {,tor }}$, equipped with the collection $\left\{\left(J_{Z}^{*} \mathcal{F}_{Z}^{\natural}, J_{\tilde{Z}}^{*} \iota Z\right)\right\}_{Z}$.

(3) $\mathcal{H}^{q}(\mathcal{F})$ is a well-positioned sheaf over $\mathrm{Y}_{\Sigma}^{\text {tor }}$, equipped with the collection $\left\{\left(\mathcal{H}^{q}\left(\mathcal{F}_{Z}^{\natural}\right), \mathcal{H}^{q}\left(\iota_{Z}\right)\right)\right\}_{\mathrm{Z}}$, for each $q$.

Proof. By Lemma 4.1.2 and Remark 4.1.7, we may assume that each collection $\mathcal{U}_{Z}$ as in Definition 4.1.3 that we consider is adapted to both $Y$ and $Y^{\prime}$. Since the canonical morphism $Y_{\tilde{Z}}^{\prime} \rightarrow Y_{\tilde{Z}} \times_{Y_{Z}^{\sharp}} Y_{Z}^{\prime}, \sharp$ induces an isomorphism between the reduced subschemes, the lemma follow from the definitions, and (for the assertion (1)) from [3, XVII, 5.1.2].

Lemma 4.2.2. Suppose $\mathcal{F}^{\prime}$ is as in Lemma 4.2.1. Suppose T is just a point. Under the assumption (in (4) of Proposition 2.1.2) that $\Sigma$ is smooth, suppose moreover that the induced morphism $\mathrm{Y}_{C}^{\natural} \rightarrow \mathrm{Y}_{\mathrm{Z}}^{\natural}$ is smooth (which is the case if $C \rightarrow \mathrm{Z}$ is) for each $\mathrm{Z}$. Then the canonical morphisms

$$
a_{\mathrm{Y}_{\tilde{z}}}^{*} R J_{*} \mathcal{F}^{\prime} \rightarrow R J_{\tilde{Z}, *} a_{\mathrm{Y}_{\tilde{z}}^{\prime}}^{*} \mathcal{F}^{\prime}
$$

and

$$
a_{\mathrm{Y}_{\tilde{Z}}^{\natural}}^{\natural, *} R J_{\mathrm{Z}, *} \mathcal{F}_{\mathrm{Z}}^{\prime, \natural} \rightarrow R J_{\tilde{\mathrm{Z}}, *} a_{\mathrm{Y}_{\tilde{\mathrm{Z}}}^{\natural}}^{\natural, *} \mathcal{F}_{\mathrm{Z}}^{\prime, \natural}
$$

are isomorphisms in $D_{c}^{b}\left(\mathrm{Y}_{\tilde{Z}}, \Lambda\right)$, and hence the isomorphism

$$
R J_{\tilde{Z}, *} \iota_{Z}^{\prime}: R J_{\tilde{Z}, *} a_{Y_{\tilde{Z}}^{\prime}}^{*} \mathcal{F}^{\prime} \rightarrow R J_{\tilde{Z}, *} a_{Y_{\tilde{Z}}^{\prime}, *}^{\natural,} \mathcal{F}_{\bar{Z}}^{\prime, \natural}
$$

canonically induces an isomorphism

$$
\iota_{\mathrm{Z}}: a_{\mathrm{Y}_{\tilde{\mathrm{Z}}}}^{*} R J_{*} \mathcal{F}^{\prime} \stackrel{\sim}{\rightarrow} a_{\mathrm{Y}_{\tilde{\mathrm{Z}}}^{\natural}}^{\natural} R J_{\mathrm{Z}, *} \mathcal{F}_{\mathrm{Z}}^{\prime, \natural}
$$

in $D_{c}^{b}\left(\mathrm{Y}_{\tilde{Z}}, \Lambda\right)$, for each Z. Moreover, $R J_{*} \mathcal{F}^{\prime}$ is a well-positioned complex over $\mathrm{Y}_{\Sigma}^{\text {tor }}$, equipped with the collection $\left\{\left(R J_{Z, *} \mathcal{F}_{Z}^{\prime, \natural}, \iota_{Z}\right)\right\}_{\mathrm{Z}}$. In particular, $R J_{*} \Lambda_{\mathrm{Y}_{\Sigma}^{\prime} \text {,tor }}$ is a well-positioned complex over $Y_{\Sigma}^{\text {tor }}$, equipped with the collection $\left\{R J_{Z, *} \Lambda_{Y_{Z}^{\prime},}\right\}_{Z}$.

Proof. For each $\mathrm{Z}$, let $\mathcal{U}_{\mathrm{Z}}=\left\{\left(\bar{U}_{i}, a_{\bar{U}_{i}}, a_{\bar{U}_{i}, E\left(\sigma_{i}\right)}^{\natural}\right)\right\}_{i \in I_{Z}}$ be any collection as in Definition 4.1.3 (for $\mathcal{F}^{\prime}$, adapted to $Y^{\prime}$ ), which we may and we shall assume to be 
also adapted to $\mathrm{Y}$, by Lemma 4.1.2 and Remark 4.1.7. For each $i \in I_{Z}$, consider the canonical morphisms

$$
\begin{gathered}
a_{\mathrm{Y}_{\bar{U}}}: \mathrm{Y}_{\bar{U}_{i}}:=\bar{U}_{i} \times_{\mathrm{X}_{\mathcal{H}, \Sigma}^{\text {tor }}} \mathrm{Y}_{\Sigma}^{\text {tor }} \rightarrow \mathrm{Y}_{\Sigma}^{\text {tor }}, \\
a_{\mathrm{Y}_{\bar{U}_{i}}^{\prime}}: \mathrm{Y}_{\bar{U}_{i}}^{\prime}:=\bar{U}_{i} \times_{\mathrm{X}_{\mathcal{H}, \Sigma}^{\text {tor }}} \mathrm{Y}_{\Sigma}^{\prime, \text { tor }} \rightarrow \mathrm{Y}_{\Sigma}^{\prime, \text { tor }}, \\
a_{\mathrm{Y}_{\bar{U}_{i}}}^{\natural}: \mathrm{Y}_{\bar{U}_{i}} \rightarrow \mathrm{Y}_{\mathrm{Z}}^{\natural},
\end{gathered}
$$

and

$$
a_{Y_{\bar{U}_{i}}^{\prime}}^{\natural}: Y_{\bar{U}_{i}}^{\prime} \rightarrow Y_{Z}^{\prime, \natural},
$$

where the restriction of

$$
\iota_{Z}^{\prime}: a_{Y_{\tilde{Z}}^{\prime}}^{*} \mathcal{F}^{\prime} \stackrel{\sim}{\rightarrow} a_{Y_{\tilde{Z}}^{\natural,}}^{\natural, *} \mathcal{F}_{Z}^{\prime, \natural}
$$

to

$$
\mathrm{Y}_{\bar{U}_{i}^{+}}^{\prime}:=\mathrm{Y}_{\bar{U}_{i}}^{\prime} \times_{\mathrm{X}_{\mathcal{H}, \Sigma}^{\text {tor }}} \tilde{\mathrm{Z}}
$$

extends to an isomorphism

$$
\iota_{\bar{U}_{i}}^{\prime}: a_{Y_{\bar{U}_{i}}^{\prime}}^{*} \mathcal{F}^{\prime} \stackrel{\sim}{\rightarrow} a_{Y_{\bar{U}_{i}}^{\prime}}^{\natural, *} \mathcal{F}_{\mathrm{Z}}^{\prime, \natural}
$$

in $D_{c}^{b}\left(\mathrm{Y}_{\bar{U}_{i}}^{\prime}, \Lambda\right)$, by assumption.

To show that (4.2.3) and (4.2.4) are isomorphisms, and that $R J_{*} \mathcal{F}^{\prime}$ is a wellpositioned complex over $Y_{\Sigma}^{\text {tor }}$, equipped with the collection $\left\{\left(R J_{Z, *} \mathcal{F}_{Z}^{\prime, \natural}, \iota_{Z}\right)\right\}_{Z}$, it suffices to fix the choices of some $i \in I_{Z}$ as above, and show that the restrictions of (4.2.3) and (4.2.4) to the open subscheme

$$
\mathrm{Y}_{\bar{U}_{i}^{+}}:=\mathrm{Y}_{\bar{U}_{i}} \times_{\mathrm{X}_{\mathcal{H}, \Sigma}^{\text {tor }}} \tilde{\mathrm{Z}}
$$

of $Y_{\tilde{Z}}$ are isomorphisms, and that the isomorphism

$$
\left.\left.\left(a_{\mathrm{Y}_{\tilde{Z}}}^{*} R J_{*} \mathcal{F}^{\prime}\right)\right|_{\bar{Y}_{\bar{U}_{i}^{+}}} \stackrel{\sim}{\rightarrow}\left(a_{\mathrm{Y}_{\tilde{z}}^{\natural}}^{\natural, *} R J_{Z, *} \mathcal{F}_{\bar{Z}}^{\prime, \natural}\right)\right|_{\bar{Y}_{\bar{U}_{i}^{+}}}
$$

(which should be the restriction of $\iota_{Z}$ to $Y_{\bar{U}_{i}^{+}}$) induced by the restrictions of (4.2.3), (4.2.4), and $R J_{\tilde{Z}, *} \iota_{Z}^{\prime}$ to $Y_{\overline{U_{i}^{+}}}$extends to an isomorphism

$$
\iota_{\bar{U}_{i}}: a_{\mathrm{Y}_{\bar{U}_{i}}}^{*} R J_{*} \mathcal{F}^{\prime} \stackrel{\sim}{\rightarrow} a_{\mathrm{Y}_{\bar{U}_{i}}^{\natural, *}}^{\natural,} R J_{\mathrm{Z}, *} \mathcal{F}_{\mathrm{Z}}^{\prime, \natural}
$$

over $\mathrm{Y}_{\bar{U}_{i}}$. Let $i_{\bar{Y}_{\bar{U}_{i}}}: \mathrm{Y}_{\bar{U}_{i}^{+}} \rightarrow \mathrm{Y}_{\bar{U}_{i}}, i_{\mathrm{Y}_{\bar{U}_{i}}^{\prime}}: \mathrm{Y}_{\bar{U}_{i}^{+}}^{\prime} \rightarrow \mathrm{Y}_{\bar{U}_{i}}^{\prime}, J_{\bar{U}_{i}}: Y_{\bar{U}_{i}}^{\prime} \hookrightarrow \mathrm{Y}_{\bar{U}_{i}}$, and $J_{\bar{U}_{i}^{+}}: \mathrm{Y}_{\bar{U}_{i}^{+}}^{\prime} \hookrightarrow \mathrm{Y}_{\bar{U}_{i}^{+}}$denote the canonical morphisms. For these purposes, it suffices to show that the canonical morphism

$$
i_{\mathrm{Y}_{\bar{U}_{i}}}^{*} R J_{\bar{U}_{i}, *} a_{Y_{\bar{U}_{i}}^{\natural}}^{\natural, *} \mathcal{F}_{\mathrm{Z}}^{\prime, \natural} \rightarrow R J_{\bar{U}_{i}^{+}, *}^{+} i_{Y_{\bar{U}_{i}}^{\prime}}^{*} a_{Y_{\bar{U}_{i}}^{\natural, *}}^{\natural,} \mathcal{F}_{\mathrm{Z}}^{\prime, \natural}
$$

is an isomorphism, by the commutativity of the following commutative diagram 


$$
\begin{aligned}
& \left.\left.\left(a_{\mathrm{Y}_{\tilde{Z}}}^{*} R J_{*} \mathcal{F}^{\prime}\right)\right|_{\bar{Y}_{\bar{U}_{i}^{+}}} \longrightarrow\left(R J_{\tilde{\mathrm{Z}}, *} a_{\mathrm{Y}_{\tilde{Z}}^{\prime}}^{*} \mathcal{F}^{\prime}\right)\right|_{\mathrm{Y}_{\bar{U}_{i}^{+}}} \\
& \text {can. } \downarrow^{2} \quad \text { can. } \downarrow^{2} \\
& i_{\mathrm{Y}_{\bar{U}_{i}}}^{*} a_{\mathrm{Y}_{\bar{U}_{i}}}^{*} R J_{*} \mathcal{F}^{\prime} \stackrel{\text { b.c. }}{\sim} i_{\mathrm{Y}_{\bar{U}_{i}}}^{*} R J_{\bar{U}_{i}, *} a_{\mathrm{Y}_{\bar{U}_{i}}^{\prime}}^{*} \mathcal{F}^{\prime} \longrightarrow R J_{\bar{U}_{i}, *}^{+} i_{\mathrm{Y}_{\bar{U}_{i}}^{\prime}}^{*} a_{\mathrm{Y}_{\bar{U}_{i}}^{\prime}}^{*} \mathcal{F}^{\prime} \\
& \left.i_{\bar{U}_{i}}^{*} R J_{\bar{U}_{i}, *} \iota_{\bar{U}_{i}}^{\prime} \downarrow^{2} \quad R J_{\bar{U}_{i}^{+}, *}\left(\iota_{\mathrm{Z}}^{\prime} \mid \mathrm{Y}_{\bar{U}_{i}^{+}}\right)\right|^{2} \\
& i_{\mathrm{Y}_{\bar{U}_{i}}}^{*} a_{\mathrm{Y}_{\bar{U}_{i}}}^{\natural, *} R J_{\mathrm{Z}, *} \mathcal{F}_{\mathrm{Z}}^{\prime, \natural} \stackrel{\text { b.c. }}{\sim} i_{\mathrm{Y}_{\bar{U}_{i}}}^{*} R J_{\bar{U}_{i}, *} a_{\mathrm{Y}_{\bar{U}_{i}}^{\prime}}^{\natural, *} \mathcal{F}_{\mathrm{Z}}^{\prime, \natural} \stackrel{(4.2 .6)}{\longrightarrow} R J_{\bar{U}_{i}^{+}, *} i_{\mathrm{Y}_{\bar{U}_{i}}^{\prime}}^{*} a_{\mathrm{Y}_{\bar{U}_{i}}^{\prime}}^{\natural, *} \mathcal{F}_{\mathrm{Z}}^{\prime, \natural} \\
& \text { can. } \downarrow^{2} \\
& \left.\left.\left(a_{\mathrm{Y}_{\tilde{Z}}^{\natural, *}}^{\natural} R J_{\mathrm{Z}, *} \mathcal{F}_{\mathrm{Z}}^{\prime, \natural}\right)\right|_{\bar{Y}_{\bar{U}_{i}^{+}}} \longrightarrow\left(R J_{\tilde{Z}, *} a_{\mathrm{Y}_{\tilde{Z}}^{\prime}}^{\natural, *} \mathcal{F}_{\mathrm{Z}}^{\prime, \natural}\right)\right|_{\mathrm{Y}_{\bar{U}_{i}^{+}}}
\end{aligned}
$$

in $D_{c}^{b}\left(\mathrm{Y}_{\bar{U}_{i}^{+}}, \Lambda\right)$, in which 'b.c.' means base change morphisms, which (under the smoothness assumptions) are isomorphisms by the smooth base change theorem (see [3, XVI, 1.2]); and in which the composition of the three vertical arrows at the rightmost column is the restriction of $R J_{\tilde{Z}, *} \ell_{Z}^{\prime}$ to the open subscheme $Y_{\bar{U}_{i}^{+}}$of $Y_{\tilde{Z}}$.

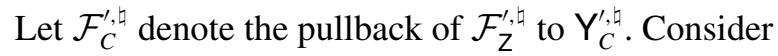

$$
\begin{gathered}
E\left(\sigma_{i}\right)^{+}:=\bigcup_{\tau \in \Sigma_{\mathrm{Z}}^{+}, \bar{\tau} \subset \overline{\sigma_{i}}} E_{\tau} \subset E\left(\sigma_{i}\right), \\
\mathrm{Y}_{E\left(\sigma_{i}\right)^{+}}^{\prime, \natural}:=E\left(\sigma_{i}\right)^{+} \times_{\operatorname{Spec}(\mathbb{Z})} \mathrm{Y}_{C}^{\prime, \natural},
\end{gathered}
$$

and

$$
\mathrm{Y}_{E\left(\sigma_{i}\right)^{+}}^{\natural}:=E\left(\sigma_{i}\right)^{+} \times_{\operatorname{Spec}(\mathbb{Z})} \mathrm{Y}_{C}^{\natural} .
$$

Let $J_{C}: \mathrm{Y}_{C}^{\prime, \natural} \rightarrow \mathrm{Y}_{C}^{\natural}$ and $J_{E\left(\sigma_{i}\right)^{+}}: \mathrm{Y}_{E\left(\sigma_{i}\right)^{+}}^{\prime, \natural} \rightarrow \mathrm{Y}_{E\left(\sigma_{i}\right)^{+}}^{\natural}$ denote the canonical morphisms. Since $\mathrm{Y}_{\bar{U}_{i}^{+}} \cong E\left(\sigma_{i}\right)^{+} \times_{E\left(\sigma_{i}\right)} \mathrm{Y}_{\bar{U}_{i}}$ and $\mathrm{Y}_{\bar{U}_{i}^{+}}^{\prime} \cong E\left(\sigma_{i}\right)^{+} \times_{E\left(\sigma_{i}\right)} \mathrm{Y}_{\bar{U}_{i}}^{\prime}$, in order to show that (4.2.6) is an isomorphism, by the smooth base change theorem (and the smoothness assumptions) again, it suffices to show that the canonical morphism

$$
\left(\mathrm{Y}_{E\left(\sigma_{i}\right)^{+}} \rightarrow \mathrm{Y}_{C}^{\natural}\right)^{*} R J_{C, *} \mathcal{F}_{C}^{\prime, \natural} \rightarrow R J_{E\left(\sigma_{i}\right)^{+}, *}\left(\mathrm{Y}_{E\left(\sigma_{i}\right)^{+}}^{\prime} \rightarrow \mathrm{Y}_{C}^{\prime, \natural}\right)^{*} \mathcal{F}_{C}^{\prime, \natural}
$$

is an isomorphism. Since $J_{E\left(\sigma_{i}\right)^{+}}$is by definition the product $\operatorname{Id}_{E\left(\sigma_{i}\right)_{\top}^{+}} \times_{\top} J_{C}$, the morphism (4.2.8) can be identified with the Künneth morphism

$$
\Lambda_{E\left(\sigma_{i}\right)_{\top}^{+}} \bigotimes_{\mathrm{T}}^{L}\left(R J_{C, *} \mathcal{F}_{C}^{\prime, \natural}\right) \rightarrow R\left(\operatorname{Id}_{E\left(\sigma_{i}\right)_{\top}^{+}} \times_{\mathrm{T}} J_{C}\right)_{*}\left(\Lambda_{E\left(\sigma_{i}\right)_{\top}^{+}} \bigotimes_{\mathrm{T}}^{L} \mathcal{F}_{C}^{\prime, \natural}\right),
$$

which is an isomorphism, because $\mathrm{T}$ is a point, by [6, 4.2.7], as desired. 
LEMMA 4.2.10. Suppose that $\mathrm{T}=\mathrm{S}$ is Henselian, that $\mathrm{Y}^{\prime}=\mathrm{Y}_{\eta}$ and $\mathrm{Y}_{Z}^{\prime, \natural}=\left(\mathrm{Y}_{Z}^{\natural}\right)_{\eta}$, and that $\mathcal{F}^{\prime}$ is as in Lemma 4.2.1. Consider the nearby cycle functors $R \Psi_{Y}$ and $R \Psi_{\mathrm{Y}_{\mathrm{Z}}^{\natural}}$, for all $\mathrm{Z}$, defined by some compatible choices of geometric points $\bar{\eta}$ and $\bar{s}$ above the generic and special points $\eta$ and $s$ of $\mathrm{S}$, respectively, as in [45, Section 5.1]. Under the assumption (in (4) of Proposition 2.1.2) that $\Sigma$ is smooth, suppose moreover that $Y_{C}^{\natural} \rightarrow Y_{Z}^{\natural}$ is smooth for each $\mathrm{Z}$. Then $R \Psi_{\Sigma}^{\operatorname{tor}}\left(\mathcal{F}^{\prime}\right)$ is a well-positioned complex over $\left(\mathrm{Y}_{\Sigma}^{\text {tor }}\right)_{\bar{s}}$, equipped with a collection $\left\{\left(R \Psi_{\mathrm{Y}_{Z}^{\sharp}}\left(\mathcal{F}_{Z}^{\prime, \natural}\right)\right.\right.$, $\left.\left.\iota_{\mathrm{Z}}\right)\right\}_{\mathrm{Z}}$, for some canonical isomorphisms $\iota_{\mathrm{Z}}$, as in Definition 4.1.3. In this case, $\iota_{\mathrm{Z}}$ can be taken to be not just in $D_{c}^{b}\left(\left(\mathrm{Y}_{\tilde{\mathrm{Z}}}\right)_{\bar{s}}, \Lambda\right)$, but also in $D_{c}^{b}\left(\left(\mathrm{Y}_{\tilde{\mathrm{Z}}}\right)_{\bar{s}} \times \bar{\eta}, \Lambda\right)$; and the isomorphisms $\iota_{\bar{U}_{i}}$ can be taken to be not just in $D_{c}^{b}\left(\left(\mathrm{Y}_{\bar{U}_{i}}\right)_{\bar{s}}, \Lambda\right)$, but also in $D_{c}^{b}\left(\left(\mathrm{Y}_{\bar{U}_{i}}\right)_{\bar{s}} \times \bar{\eta}, \Lambda\right)$. (Here $D_{c}^{b}\left((\cdot)_{\bar{s}} \times \bar{\eta}, \Lambda\right)$ denotes the bounded derived category of $\Lambda$-étale constructible sheaves over $(\cdot)_{\bar{s}}$ with compatible continuous $\operatorname{Gal}(k(\bar{\eta}) / k(\eta))$-actions. $)$

Suppose that $\Lambda=\mathbb{Q}_{\ell}$ or $\overline{\mathbb{Q}}_{\ell}$, and that $Y_{C}^{\natural} \rightarrow Y_{Z}^{\natural}$ is smooth of relative dimension $d^{\mathrm{Z}}-d_{E}$, where $d^{\mathrm{Z}}:=d-d_{\mathrm{Z}}, d:=\operatorname{dim}\left(\left(\mathbf{X}_{\mathcal{H}}\right)_{\eta}\right), d_{\mathrm{Z}}:=\operatorname{dim}\left(Z_{\eta}\right)$, and $d_{E}:=\operatorname{dim}\left(E_{\eta}\right)$, for each $\mathrm{Z}$. If $\mathcal{F}^{\prime}$ is a well-positioned perverse sheaf over $\mathrm{Y}_{\Sigma}^{\prime \text {,tor }}=\left(\mathrm{Y}_{\Sigma}^{\text {tor }}\right)_{\eta}$, and if $\mathcal{F}_{Z}^{\prime, \natural}\left[-d^{\mathrm{Z}}\right]$ is a perverse sheaf over $\mathrm{Y}_{Z}^{\prime, \natural}=\left(\mathrm{Y}_{Z}^{\natural}\right)_{\eta}$, for each $\mathrm{Z}$, then $R \Psi_{Y_{\Sigma}^{\text {tor }}}\left(\mathcal{F}^{\prime}\right)$ is a well-positioned perverse sheaf over $\left(\mathrm{Y}_{\Sigma}^{\text {tor }}\right)_{\bar{s}}$, equipped with the collection $\left\{\left(R \Psi_{Y_{Z}^{\sharp}}\left(\mathcal{F}_{Z}^{\prime, \natural}\right), \iota \mathrm{Z}\right)\right\}_{\mathrm{Z}}$ as above, where $R \Psi_{\mathrm{Y}_{\mathrm{Z}}^{\sharp}}\left(\mathcal{F}_{\mathrm{Z}}^{\prime, \natural}\right)\left[-d^{\mathrm{Z}}\right]$ is a perverse sheaf, for each $\mathrm{Z}$.

Proof. For the first paragraph of the lemma, by the same reduction steps as in the proof of Lemma 4.2.2, we are reduced to showing that the canonical morphism

$$
\Lambda_{E\left(\sigma_{i}\right)_{s}^{+}} \bigotimes_{\bar{s}}^{L}\left(R \Psi_{Y_{C}^{\sharp}}\left(\mathcal{F}_{C}^{\prime, \natural দ}\right)\right) \rightarrow R \Psi_{E\left(\sigma_{i}\right)_{\mathrm{S}}^{+} \times_{\mathrm{S}} Y_{C}^{\sharp}}\left(\Lambda_{E\left(\sigma_{i}\right)_{\eta}^{+}} \nabla_{\eta}^{L} \mathcal{F}_{C}^{\prime, \natural}\right)
$$

(cf. (4.2.9)) in $D_{c}^{b}\left(\left(E\left(\sigma_{i}\right)_{\mathrm{S}}^{+} \times_{S} Y_{C}^{\natural}\right)_{\bar{s}} \times \bar{\eta}, \Lambda\right)$ is an isomorphism, where $\mathcal{F}_{C}^{\prime, \text {, }}$ denotes the pullback of $\mathcal{F}_{Z}^{\prime, \natural}$ under $Y_{C}^{\prime, \natural} \rightarrow Y_{Z}^{\prime, \natural}$, for each $Z$. Since $\sigma_{i}$ is smooth by assumption, $E\left(\sigma_{i}\right)^{+}$is a relative normal crossings divisor on the smooth scheme $E\left(\sigma_{i}\right)$ over $\operatorname{Spec}(\mathbb{Z})$. Hence, by [16, XIII, 2.1.11] (or rather its proof), we have a canonical isomorphism

$$
\Lambda_{E\left(\sigma_{i}\right)_{\bar{s}}^{+}} \stackrel{\sim}{\rightarrow} R \Psi_{E\left(\sigma_{i}\right)_{\mathrm{S}}^{+}}\left(\Lambda_{E\left(\sigma_{i}\right)_{\eta}^{+}}\right)
$$

in $D_{c}^{b}\left(E\left(\sigma_{i}\right)_{\bar{s}}^{+} \times \bar{\eta}, \Lambda\right)$, via which (4.2.11) induces the Künneth morphism

$$
\left(R \Psi_{E\left(\sigma_{i}\right)_{\mathrm{S}}^{+}}\left(\Lambda_{E\left(\sigma_{i}\right)_{\eta}^{+}}\right)\right) \bigotimes_{\bar{s}}^{L}\left(R \Psi_{Y_{C}^{\sharp}}\left(\mathcal{F}_{C}^{\prime, \natural}\right)\right) \rightarrow R \Psi_{E\left(\sigma_{i}\right)_{S}^{+} \times_{S} Y_{C}^{\natural}}\left(\Lambda_{E\left(\sigma_{i}\right)_{\eta}^{+}} \bigotimes_{\eta}^{L} \mathcal{F}_{C}^{\prime, \natural}\right) .
$$

Thus, (4.2.11) is an isomorphism because (4.2.12) is, by [27, 4.7]. The second paragraph of the lemma then follows from $[27,4.5]$ and $[6,4.2 .8]$. 
Lemma 4.2.13. Suppose that $\mathrm{T}=\operatorname{Spec}(k)$ for some field $k$ that is either algebraically closed or finite, and that $\Lambda=\mathbb{Q}_{\ell}$ or $\overline{\mathbb{Q}}_{\ell}$. Suppose that $\left(\mathrm{X}_{\mathcal{H}}\right)_{\mathrm{T}}$ and $\mathrm{Z}_{\mathrm{T}}$ are equidimensional of dimensions $d$ and $d_{\mathrm{Z}}$, respectively, for each $\mathrm{Z}$. Under the assumption (in (4) of Proposition 2.1.2) that $\Sigma$ is smooth, suppose moreover that $Y_{C}^{\natural} \rightarrow Y_{Z}^{\natural}$ is smooth of relative dimension $d^{Z}-d_{E}$, where $d^{Z}:=d-d_{Z}$ and $d_{E}:=\operatorname{dim}\left(E_{\mathrm{T}}\right)$, for each $Z$. Suppose $\mathcal{F}^{\prime}$ is a well-positioned perverse sheaf over $Y_{\Sigma}^{\prime \text {,tor }}$, equipped with a collection $\mathcal{F}^{\prime, \natural}=\left\{\mathcal{F}_{Z}^{\prime, \natural}\right\}_{Z}$, where $\mathcal{F}_{Z}^{\prime, \natural}\left[-d^{\mathrm{Z}}\right]$ is a perverse sheaf over $\mathrm{Y}_{Z}^{\prime, \natural}$, for each $\mathrm{Z}$. Then $a_{\mathrm{Y}_{\bar{Z}}^{\prime}}^{*} \mathcal{F}^{\prime}[-1]$ and $a_{\mathrm{Y}_{\bar{Z}}^{\prime}}^{\natural, *} \mathcal{F}_{Z}^{\prime, \natural}[-1]$ are perverse sheaves (isomorphic to each other under $\left.\iota_{\mathrm{Z}}^{\prime}[-1]\right)$ in $D_{c}^{b}\left(\mathrm{Y}_{\tilde{\mathrm{Z}}}^{\prime}, \Lambda\right)$; and $a_{\mathrm{Y}_{\tilde{\mathrm{z}}}^{*}}^{*}\left(J_{! *} \mathcal{F}^{\prime}\right)[-1]$ and $a_{Y_{\tilde{Z}}^{\natural}}^{\natural, *} J_{Z, * *}\left(\mathcal{F}_{\bar{Z}}^{\prime, \natural}\left[-d^{Z}\right]\right)\left[d^{Z}-1\right]$ are perverse sheaves in $D_{c}^{b}\left(Y_{\tilde{Z}}, \Lambda\right)$. Moreover, we have canonical isomorphisms

$$
a_{\Upsilon_{\tilde{z}}}^{*}\left(J_{! *} \mathcal{F}^{\prime}\right)[-1] \stackrel{\sim}{\rightarrow} J_{\tilde{Z}, ! *}\left(a_{Y_{\tilde{Z}}^{\prime}}^{*} \mathcal{F}^{\prime}[-1]\right)
$$

and

$$
a_{\Upsilon_{\tilde{z}}^{\natural, *}}^{\natural}\left(J_{Z, ! *}\left(\mathcal{F}_{\bar{Z}}^{\prime, \natural}\left[-d^{\mathrm{Z}}\right]\right)\right)\left[d^{\mathrm{Z}}-1\right] \stackrel{\sim}{\rightarrow} J_{\tilde{Z}, ! *}\left(a_{Y_{\tilde{Z}}^{\natural}}^{\natural, *} \mathcal{F}_{\mathrm{Z}}^{\prime, \natural}[-1]\right)
$$

in $D_{c}^{b}\left(\mathrm{Y}_{\tilde{Z}}, \Lambda\right)$ extending the compositions of canonical isomorphisms

$$
J_{\tilde{\mathrm{Z}}}^{*} a_{\mathrm{Y}_{\tilde{z}}}^{*}\left(J_{! *} \mathcal{F}^{\prime}\right)[-1] \stackrel{\sim}{\rightarrow} a_{\mathrm{Y}_{\bar{z}}^{\prime}}^{*} J^{*}\left(J_{! *} \mathcal{F}^{\prime}\right)[-1] \stackrel{\sim}{\rightarrow} a_{Y_{\tilde{z}}^{\prime}}^{*} \mathcal{F}^{\prime}[-1]
$$

and

$$
\begin{aligned}
& J_{\tilde{Z}}^{*} a_{Y_{\tilde{Z}}^{\natural, *}}^{\natural}\left(J_{Z, ! *}\left(\mathcal{F}_{Z}^{\prime, \natural}\left[-d^{Z}\right]\right)\right)\left[d^{Z}-1\right] \stackrel{\sim}{\rightarrow} a_{Y_{\tilde{Z}}^{\natural}}^{\natural, *} J_{Z}^{*}\left(J_{Z, ! *}\left(\mathcal{F}_{Z}^{\prime, \natural}\left[-d^{Z}\right]\right)\right)\left[d^{Z}-1\right] \\
& \stackrel{\sim}{\rightarrow} a_{Y_{\tilde{Z}}^{\natural}, *}^{, \prime} \mathcal{F}_{Z}^{\prime, \natural}[-1],
\end{aligned}
$$

respectively, in $D_{c}^{b}\left(\mathrm{Y}_{\tilde{Z}}^{\prime}, \Lambda\right)$. Hence, the isomorphism

$$
J_{\tilde{Z}, ! *}\left(\iota_{Z}^{\prime}[-1]\right)[1]: J_{\tilde{Z},: *}\left(a_{Y_{\tilde{Z}}^{\prime}}^{*} \mathcal{F}^{\prime}[-1]\right)[1] \stackrel{\sim}{\rightarrow} J_{\tilde{Z}, ! *}\left(a_{Y_{\tilde{Z}}^{\prime}}^{\natural, *} \mathcal{F}_{Z}^{\prime, \natural}[-1]\right)[1]
$$

canonically induces an isomorphism

$$
\iota \mathrm{Z}: a_{\mathrm{Y}_{\tilde{Z}}}^{*} J_{! *} \mathcal{F}^{\prime} \stackrel{\sim}{\rightarrow} a_{\Upsilon_{\tilde{Z}}^{\natural}}^{\natural} J_{Z, ! *}\left(\mathcal{F}_{Z}^{\prime, \natural}\left[-d^{\mathrm{Z}}\right]\right)\left[d^{\mathrm{Z}}\right]
$$

in $D_{c}^{b}\left(\mathrm{Y}_{\tilde{Z}}, \Lambda\right)$, for each $\mathrm{Z}$; and $J_{! *} \mathcal{F}^{\prime}$ is a well-positioned perverse sheaf over $\mathrm{Y}_{\Sigma}^{\text {tor }}$, equipped with the collection $\left.\left\{\left(J_{Z, ! *}\left(\mathcal{F}_{Z}^{\prime, \natural}\left[-d^{\mathrm{Z}}\right]\right)\right)\left[d^{\mathrm{Z}}\right], \iota_{\mathrm{Z}}\right)\right\}_{\mathrm{Z}}$.

In particular, suppose $\mathrm{Y}^{\prime}=\mathrm{Y}_{\mathrm{sm}}$ and $\mathrm{Y}_{\mathrm{Z}}^{\prime, \natural}=\left(\mathrm{Y}_{\mathrm{Z}}^{\natural}\right)_{\mathrm{sm}}$ in the above, which are valid choices by Lemma 2.2.10, so that $\mathrm{Y}_{\Sigma}^{\prime}$,tor $=\left(\mathrm{Y}_{\Sigma}^{\text {tor }}\right)_{\mathrm{sm}}$. Consider the intersection complexes $\underline{\mathcal{I}}_{Y_{\Sigma}^{\text {tor }}}:=J_{! *}\left(\Lambda_{\left(\mathrm{Y}_{\Sigma}^{\text {tor }}\right)_{\mathrm{sm}}}[d]\right)$ and $\underline{\mathcal{I}}_{\mathrm{Y}_{\mathrm{Z}}^{\sharp}}:=J_{\mathrm{Z}, ! *}\left(\Lambda_{\mathrm{Y}_{\mathrm{Z}, \mathrm{sm}}^{\sharp}}\left[d_{\mathrm{Z}}\right]\right)$. Then $\underline{\mathcal{I C}}_{Y_{\Sigma}^{\text {tor }}}$ is a well-positioned perverse sheaf, equipped with the collection $\left\{\mathcal{I C}_{\mathrm{Y}_{\mathrm{Z}}^{\sharp}}\left[d^{\mathrm{Z}}\right]\right\}_{\mathrm{Z}}$. 
Proof. In addition to showing that $a_{Y_{\bar{Z}}^{\prime}}^{*} \mathcal{F}^{\prime}[-1], a_{Y_{\bar{Z}}^{\natural}}^{\natural, *} \mathcal{F}_{\bar{Z}}^{\prime, \natural}[-1], a_{Y_{\hat{z}}}^{*}\left(J_{! *} \mathcal{F}^{\prime}\right)[-1]$, and $a_{Y_{\tilde{z}}}^{\natural, *} J_{Z, ! *}\left(\mathcal{F}_{Z}^{\prime, \natural}\left[-d^{Z}\right]\right)\left[d^{Z}-1\right]$ are all perverse sheaves, since the assignment of middle perversity extensions is functorial and fully faithful (see [29, Corollary III.5.11]), in order to also show that the compositions of canonical isomorphisms (4.2.16) and (4.2.17) (necessarily uniquely) extend to the desired canonical isomorphisms (4.2.14) and (4.2.15), so that all remaining assertions of the lemma will follow, it suffices to show that the perverse sheaves $a_{\hat{Y}_{\hat{z}}}^{*}\left(J_{! *} \mathcal{F}^{\prime}\right)[-1]$ and $a_{Y_{\tilde{z}}}^{\natural, *} J_{Z, ! *}\left(\mathcal{F}_{Z}^{\prime, \natural}\left[-d^{Z}\right]\right)\left[d^{Z}-1\right]$ are isomorphic to the middle perversity extensions of their restrictions to $Y_{\tilde{z}}^{\prime}$, respectively. Essentially by definition (see also [29, the criterion on page 148, after Lemma-Definition III.5.2]), both the perversity and this last property can be verified étale locally.

For each $Z$, let $\mathcal{U}_{\mathbf{Z}}=\left\{\left(\bar{U}_{i}, a_{\bar{U}_{i}}, a_{\bar{U}_{i}, E\left(\sigma_{i}\right)}^{\natural}\right)\right\}_{i \in I_{Z}}$ be as in Definition 4.1.3 (for $\mathcal{F}^{\prime}$, adapted to $Y^{\prime}$ ), which we may and we shall assume to be also adapted to $Y$, by Lemma 4.1.2 and Remark 4.1.7. Since $\mathcal{U}_{Z}$ induces an affine open covering of $\tilde{Z}$ by assumption, by the explanation in the previous paragraph, and by using the canonical isomorphisms induced by $\iota_{Z}^{\prime}$ and $\iota_{\bar{U}_{i}}^{\prime}$, for each $i \in I_{Z}$, it suffices to show that $i_{\mathrm{Y}_{\overline{U_{i}}}^{\prime}}^{*} a_{\mathrm{Y}_{\bar{U}_{i}}^{\natural}}^{\natural, *} \mathcal{F}_{\mathrm{Z}}^{\prime, \natural}[-1]$ is a perverse sheaf in $D_{c}^{b}\left(\mathrm{Y}_{\bar{U}_{i}^{+}}^{\prime}, \Lambda\right)$, and that the composition of canonical isomorphisms

$$
\begin{aligned}
& J_{\bar{U}_{i}^{+}}^{*} i_{\mathrm{Y}_{\bar{U}_{i}}}^{*}\left(J_{\bar{U}_{i}, l *} a_{\mathrm{Y}_{\bar{U}_{i}}^{\prime}}^{\natural, *} \mathcal{F}_{\mathrm{Z}}^{\prime, \natural}\right)[-1] \stackrel{\sim}{\rightarrow} i_{\mathrm{Y}_{\bar{U}_{i}}^{\prime}}^{*} J_{\bar{U}_{i}}^{*}\left(J_{\bar{U}_{i}, ! *} a_{\mathrm{Y}_{\bar{U}_{i}}^{\prime}}^{\natural, *} \mathcal{F}_{\mathrm{Z}}^{\prime, \natural}\right)[-1] \\
& \stackrel{\sim}{\rightarrow} i_{\mathrm{Y}_{\bar{U}_{i}}^{\prime}}^{*} a_{\mathrm{Y}_{\bar{U}_{i}}^{\prime}}^{\natural, *} \mathcal{F}_{\mathrm{Z}}^{\prime, \natural}[-1] \text {. }
\end{aligned}
$$

in $D_{c}^{b}\left(\mathrm{Y}_{\bar{U}_{i}^{+}}^{\prime}, \Lambda\right)$ (necessarily uniquely) extends to some isomorphism

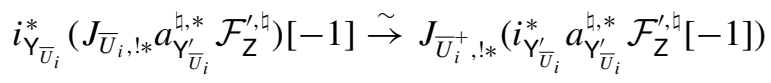

in $D_{c}^{b}\left(\mathrm{Y}_{\bar{U}_{i}^{+}}, \Lambda\right)$. (Then $i_{\mathrm{Y}_{\bar{U}_{i}}}^{*}\left(J_{\bar{U}_{i}, ! *} a_{\mathrm{Y}_{\bar{U}_{i}}^{\natural}}^{\natural, *} \mathcal{F}_{Z}^{\prime, \natural}\right)[-1]$ is a perverse sheaf in $D_{c}^{b}\left(\mathrm{Y}_{\bar{U}_{i}^{+}}, \Lambda\right)$ that is isomorphic to the middle perversity extension of its restriction to $\mathrm{Y}_{\bar{U}_{i}^{+}}^{\prime}$.)

Under the assumption that $\Sigma$ is smooth, $E\left(\sigma_{i}\right)^{+}$is a relative normal crossings divisor on the smooth scheme $E\left(\sigma_{i}\right)$ over $\operatorname{Spec}(\mathbb{Z})$, and hence $\Lambda_{E\left(\sigma_{i}\right)_{\dagger}^{+}}\left[d_{E}-1\right]$ is a perverse sheaf over $E\left(\sigma_{i}\right)_{T}^{+}$, by [29, Lemma III.6.5]. Since $\mathcal{F}_{Z}^{\prime, \natural}\left[-d^{Z}\right]$ is a perverse sheaf, under the assumption that $Y_{C}^{\natural} \rightarrow Y_{Z}^{\natural}$ is smooth of relative dimension $d^{Z}-$ $d_{E}$, by the smooth base change theorem (see [3, XVI, 1.2]) and by the $t$-exactness of smooth morphisms up to shifting by the relative dimensions (see $[6,4.2 .5]$ ),

$$
\mathcal{F}_{C}^{\prime, \natural}\left[-d_{E}\right]:=\left(\mathrm{Y}_{C}^{\prime, \natural} \rightarrow \mathrm{Y}_{Z}^{\prime, \natural}\right)^{*} \mathcal{F}_{Z}^{\prime, \natural}\left[-d_{E}\right]
$$


is also a perverse sheaf. Since $a_{\mathrm{Y}_{\bar{U}_{i}}^{\prime}}^{\natural}$ and $a_{\bar{Y}_{\bar{U}_{i}}}^{\natural}$ are pullbacks of the composition of the étale morphism $a_{\bar{U}_{i}, E\left(\sigma_{i}\right)}^{\natural}: \bar{U}_{i} \rightarrow E\left(\sigma_{i}\right) \times_{\operatorname{Spec}(\mathbb{Z})} C$ with the canonical morphism $E\left(\sigma_{i}\right) \times_{\operatorname{Spec}(\mathbb{Z})} C \rightarrow \mathrm{Z}$, and since $i_{Y_{\bar{U}_{i}^{\prime}}^{\prime}}$ and $i_{Y_{\bar{U}_{i}}}$ are pullbacks of the closed immersion $E\left(\sigma_{i}\right)^{+} \rightarrow E(\sigma)$, it follows that $i_{\mathrm{Y}_{\bar{U}_{i}}^{\prime}}^{*} a_{\mathrm{Y}_{\bar{U}_{i}}^{\prime}}^{\text {, ** }} \mathcal{F}_{\mathrm{Z}}^{\prime, 4}[-1]$ and $i_{\mathrm{Y}_{\bar{U}_{i}}}^{*}\left(J_{\bar{U}_{i}, ! *} a_{\mathrm{Y}_{\bar{U}_{i}}^{\natural, *}}^{\bar{F}_{Z}^{*}} \mathcal{F}_{Z}^{\prime, \natural}\right)[-1]$ are perverse sheaves in $D_{c}^{b}\left(\mathrm{Y}_{\bar{U}_{i}^{+}}^{\prime}, \Lambda\right)$ and $D_{c}^{b}\left(\mathrm{Y}_{\bar{U}_{i}^{+}}, \Lambda\right)$, respectively, because they are compatibly isomorphic to the pullbacks under compatible étale morphisms of the perverse sheaves

$$
\left(\Lambda_{E\left(\sigma_{i}\right)_{\top}^{+}}\left[d_{E}-1\right]\right) \otimes_{\mathrm{T}}^{L}\left(\mathcal{F}_{C}^{\prime, \natural}\left[-d_{E}\right]\right)
$$

and

$$
\left(\Lambda_{E\left(\sigma_{i}\right)_{\top}^{+}}\left[d_{E}-1\right]\right) \otimes_{\mathrm{\top}}^{L}\left(J_{C, ! *}\left(\mathcal{F}_{C}^{\prime, \natural}\left[-d_{E}\right]\right)\right)
$$

(see $[6,4.2 .8]$ ), respectively; and that (via these compatible isomorphisms) the composition (4.2.19) can be identified with the pullback of the canonical isomorphism

$$
\begin{aligned}
& \left(\operatorname{Id}_{E\left(\sigma_{i}\right)_{\top}^{+}} \times_{\mathrm{T}} J_{C}\right)^{*}\left(\left(\Lambda_{E\left(\sigma_{i}\right)_{\mathrm{\top}}^{+}}\left[d_{E}-1\right]\right) \bigotimes_{\mathrm{T}}^{L}\left(J_{C, ! *}\left(\mathcal{F}_{C}^{\prime, \natural}\left[-d_{E}\right]\right)\right)\right) \\
& \quad \stackrel{\sim}{\rightarrow}\left(\Lambda_{E\left(\sigma_{i}\right)_{\top}^{+}}\left[d_{E}-1\right]\right) \bigotimes_{\mathrm{T}}^{L}\left(\mathcal{F}_{C}^{\prime, \natural}\left[-d_{E}\right]\right) .
\end{aligned}
$$

Since (4.2.21) extends to the Künneth isomorphism

$$
\begin{aligned}
& \left(\Lambda_{E\left(\sigma_{i}\right)_{\top}^{+}}\left[d_{E}-1\right]\right) \bigotimes_{\mathrm{T}}^{L}\left(J_{C, ! *}\left(\mathcal{F}_{C}^{\prime, \text { घ }}\left[-d_{E}\right]\right)\right) \\
& \quad \stackrel{\sim}{\rightarrow}\left(\operatorname{Id}_{E\left(\sigma_{i}\right)_{\top}^{+}} \times_{\mathrm{T}} J_{C}\right)_{! *}\left(\left(\Lambda_{E\left(\sigma_{i}\right)_{\top}^{+}}\left[d_{E}-1\right]\right) \bigotimes_{\mathrm{T}}^{L}\left(\mathcal{F}_{C}^{\prime, \natural}\left[-d_{E}\right]\right)\right)
\end{aligned}
$$

(see [6, 4.2.8] again), it follows that (4.2.19) also extends to some isomorphism (4.2.20), which can be identified with the pullback of (4.2.22), as desired.

Lemma 4.2.23. Suppose that $\mathrm{S}$ is Henselian, and that $\Lambda=\mathbb{Q}_{\ell}$ or $\overline{\mathbb{Q}}_{\ell}$ for simplicity. Consider the nearby cycle functors $R \Psi_{\mathrm{X}_{\mathcal{H}, \Sigma}^{\text {tor }}}$ and $R \Psi_{\mathrm{Z}}$, for all Z, defined by some compatible choices of geometric points $\bar{\eta}$ and $\bar{s}$ above the generic and special points $\eta$ and $s$ of $\mathrm{S}$, respectively, as in [45, Section 5.1]. Under the assumption (in (4) of Proposition 2.1.2) that $\Sigma$ is smooth, suppose moreover that $C \rightarrow Z$ has connected geometric fibers and is smooth of relative dimension $d^{\mathrm{Z}}-$ $d_{E}$, where $d:=\operatorname{dim}\left(\left(\mathrm{X}_{\mathcal{H}}\right)_{\eta}\right), d_{\mathrm{Z}}:=\operatorname{dim}\left(\mathrm{Z}_{\eta}\right), d^{\mathrm{Z}}:=d-d_{\mathrm{Z}}$, and $d_{E}=\operatorname{dim}\left(E_{\mathrm{T}}\right)$, for each Z, so that Assumption 3.7.7 holds. Suppose that $Y \in \operatorname{Supp}\left(R \Psi_{\mathrm{X}_{\mathcal{H}, \Sigma}^{\text {tor }}}(\Lambda[d])\right)$ and $\mathcal{G} \in \operatorname{Loc}_{Y}\left(R \Psi_{\chi_{\mathcal{H}, \Sigma}^{\text {tor }}}(\Lambda[d])\right)$, with corresponding $Y_{Z}^{\natural} \in \operatorname{Supp}\left(R \Psi_{Z}\left(\Lambda\left[d_{Z}\right]\right)\right)$ and $\mathcal{G}_{Z}^{\natural} \in \operatorname{Loc}_{Y_{Z}^{\natural}}\left(R \Psi_{Z}\left(\Lambda\left[d_{Z}\right]\right)\right)$, for each Z, as in Lemma 3.7.9 and Proposition 3.7.13. Then $Y \cap\left(\mathrm{X}_{\mathcal{H}}\right)_{\bar{s}}$ is a well-positioned subset of $\left(\mathrm{X}_{\mathcal{H}}\right)_{\bar{s}}$, by Proposition 3.7.13, 
with its toroidal compactification $Y$ as in Definition 2.3.1. Moreover, there exist smooth open subschemes $Y^{0}$ and $Y_{\mathrm{Z}}^{\natural, 0}$ of $Y$ and $Y_{\mathrm{Z}}^{\natural}$ (with their reduced subscheme structures), respectively, for each $\mathrm{Z}$, over which $\mathcal{G}$ and $\mathcal{G}_{Z}^{\natural}$ are defined, such that $Y^{0} \cap\left(\mathrm{X}_{\mathcal{H}}\right)_{\bar{s}}$ is a well-positioned subset of $\left(\mathrm{X}_{\mathcal{H}}\right)_{\bar{s}}$, with its toroidal compactification $Y^{0}$ as in Definition 2.3.1. Let $J_{Y}: Y^{0} \hookrightarrow Y$ and $J_{Y_{Z}^{\natural}}: Y_{Z}^{\natural, 0} \hookrightarrow Y_{Z}^{\natural}$ denote the canonical open immersions, for each Z. By [20, Appendix, Theorem 10.1] and [6, 5.3.1, 5.3.2, and 5.3.4], $\underline{\mathcal{I C}}_{Y}(\mathcal{G}):=J_{Y, * *}(\mathcal{G}[\operatorname{dim}(Y)])\left(\right.$ respectively $\underline{\mathcal{I C}}_{Y_{Z}^{\natural}}\left(\mathcal{G}_{Z}^{\natural}\right):=$ $J_{Y_{Z}^{\natural} ! *}\left(\mathcal{G}_{Z}^{\natural}\left[\operatorname{dim}\left(Y_{Z}^{\natural}\right)\right]\right)$, for each $\left.Z\right)$ is a pure perverse sheaf. By [6, 5.3.5 and 5.3.8], the $\underline{\mathcal{I C}}_{Y}(\mathcal{G})$-isotypic subquotient $\mathcal{H}$ of $R \Psi_{\mathrm{X}_{\mathcal{H}, \Sigma}^{\mathrm{tor}}}(\Lambda[d])$ (respectively $\underline{\mathcal{I C}}_{Y_{Z}^{\natural}}\left(\mathcal{G}_{Z}^{\natural}\right)\left[d^{\mathrm{Z}}\right]$ isotypic subquotient $\mathcal{H}_{\mathrm{Z}}^{\natural}$ of $\left.R \Psi_{\mathrm{Z}}(\Lambda[d])\right)$ is defined. Then $\mathcal{H}$ is a well-positioned pure perverse sheaf over $Y$, equipped with a collection $\left\{\left(\mathcal{H}_{\mathrm{Z}}^{\natural}, \iota_{\mathrm{Z}}\right)\right\}_{\mathrm{Z}}$, where $\iota_{\mathrm{Z}}$ is induced by its analogue for nearby cycles as in Lemma 4.2.10, for each Z.

Proof. These follow from Proposition 3.7.13, from Lemmas 2.2.10, 4.2.10, and 4.2.13 and their proofs, and from the following: For each Z, and for each collection $\mathcal{U}_{\mathrm{Z}}=\left\{\left(\bar{U}_{i}, a_{\bar{U}_{i}}, a_{\bar{U}_{i}, E\left(\sigma_{i}\right)}^{\natural}\right)\right\}_{i \in I_{Z}}$ as in Definition 4.1.3, the isomorphisms $\iota_{Z}$ and $\iota_{\bar{U}_{i}}$, for all $i \in I_{Z}$, are induced by their analogues for nearby cycles, because the corresponding isotypical subquotients of nearby cycles match over $\bar{U}_{i}$, for all $i \in I_{\mathrm{Z}}$, and because the fibered category of perverse sheaves is a stack (see [6, 2.2.19]).

EXAMPLE 4.2.24. (This is a more detailed version of a remark we made in the introduction.) Suppose that we are in the setting of Section 3.4. For simplicity, assume that $\mathcal{O} \otimes_{\mathbb{Z}} \mathbb{Q}$ is simple and involves no factor of type $\mathrm{D}$, in the sense of [36, Definition 1.2.1.15], so that $G \otimes_{\mathbb{Z}} \mathbb{Q}$ is connected. Suppose that

$$
\mathcal{H}=\mathcal{U}\left(n_{0} p^{2018}\right):=\operatorname{ker}\left(\mathrm{G}(\hat{\mathbb{Z}}) \rightarrow \mathrm{G}\left(\mathbb{Z} /\left(n_{0} p^{2018} \mathbb{Z}\right)\right)\right),
$$

for some $n_{0} \geqslant 3$. By Lemma 2.1.9, $C \rightarrow Z$ is an abelian scheme, for each Z. Consider any Newton stratum $\left(\mathrm{X}_{\mathcal{H}}\right)_{s}^{b}$, for some $b \in \mathrm{B}\left(\mathrm{G} \otimes_{\mathbb{Z}} \mathbb{Q}_{p}\right)$, as in Proposition 3.3.1, and any Ekedahl-Oort stratum $\left(\mathbf{X}_{\mathcal{H}}\right)_{s}^{w}$, for some $w \in W^{\mathrm{M}_{0}}$, as in Proposition 3.5.1, which are well-positioned subschemes of $\left(\mathbf{X}_{\mathcal{H}}\right)_{s}$, by Propositions 3.3.9 and 3.5.5. By Lemma 2.2.3 and Proposition 2.3.12, any irreducible component $\mathrm{Y}$ of the intersection $\left(\mathbf{X}_{\mathcal{H}}\right)_{s}^{b} \cap\left(\mathbf{X}_{\mathcal{H}}\right)_{s}^{w}$ (with its reduced subscheme structure) is a well-positioned subscheme of $\left(\mathrm{X}_{\mathcal{H}}\right)_{s}$. Thus, the (generally infinite-dimensional) coherent cohomology of its partial toroidal compactification still satisfies our generalization of Koecher's principle, by Theorem 2.5.11. Moreover, by Lemmas 4.2.1, 4.2.2, and 4.2.13, if we denote by $J: \mathrm{Y} \hookrightarrow\left(\mathrm{X}_{\mathcal{H}, \Sigma}^{\text {tor }}\right)_{s}$ the canonical immersion, then $J_{!} \overline{\mathbb{Q}}_{\ell}$ (respectively $R J_{*} \overline{\mathbb{Q}}_{\ell}$, respectively the intersection complex $\left.\underline{\mathcal{I}}_{Y_{\Sigma}^{\text {tor }}}\right)$ is a well-positioned sheaf 
(respectively complex, respectively perverse sheaf) over $\left(\mathrm{X}_{\mathcal{H}, \Sigma}^{\text {tor }}\right)_{s}$, and we have generalizations of Pink's and Morel's formulas for these in Sections 4.3 and 4.5 (see Theorems 4.3.16 and 4.5.26 below).

4.3. Pink's formula. The goal of this subsection is to generalize [72, Theorem 5.3.1]. We first state its original form for automorphic étale sheaves (see Theorem 4.3.10 below), and then its generalization for tensor products with wellpositioned complexes (see Theorem 4.3.16 below).

Let us start with some preparations. For simplicity, in this subsection, let us make the following assumptions (although some of them can be relaxed):

AsSumption 4.3.1.

(1) In all cases, assume that $\mathcal{H}=\mathcal{H}^{\ell, p} \mathcal{U}_{\ell}\left(\ell^{r}\right) \mathcal{H}_{p}$, where $\mathcal{H}^{\ell, p} \subset \mathrm{G}\left(\hat{\mathbb{Z}}^{\ell, p}\right)$ and $\mathcal{H}_{p} \subset \mathrm{G}\left(\mathbb{Z}_{p}\right)$ are open compact subgroups, and where $\mathcal{U}_{\ell}\left(\ell^{r}\right):=$ $\operatorname{ker}\left(\mathrm{G}\left(\mathbb{Z}_{\ell}\right) \rightarrow \mathrm{G}\left(\mathbb{Z} / \ell^{r} \mathbb{Z}\right)\right)$, for some $r \geqslant 1$.

(2) In Cases $(\mathrm{Sm}),(\mathrm{Nm})$, or $(\mathrm{Spl})$, also assume that $\mathcal{O} \otimes_{\mathbb{Z}} \mathbb{Q}$ involves no factor of type $\mathrm{D}$ (as in [36, Definition 1.2.1.15]), so that $\mathrm{G} \otimes_{\mathbb{Z}} \mathbb{Q}$ is connected.

(3) In Case ( $\mathrm{Sm}$ ), assume in addition that $\ell \notin \square$. (This is forced by the condition (1) above when $r>1$.)

(4) In Cases $(\mathrm{Nm}),(\mathrm{Spl})$, and ( $\mathrm{Hdg})$, assume in addition that $C \rightarrow \mathrm{Z}$ is an abelian scheme torsor (of constant relative dimension) over a finite étale cover, for each Z (cf. Remark 2.1.8). In Cases ( $\mathrm{Nm})$ and $(\mathrm{Spl})$, this is true, for example, when we assume that $\mathcal{H}$ is a principal level, and that we are in the setting preceding Lemma 2.1.9. (See also Remark 2.1.11). In Case (Hdg), this is true, for example, when $p>2$ and $\mathcal{H}_{p}$ is a maximal hyperspecial open compact subgroup of $\mathrm{G}\left(\mathbb{Q}_{p}\right)$ (see [50, Sections 4.3 and 5.3]).

LEMMA 4.3.2. Under Assumption 4.3.1, there exists a system of open compact subgroups $\mathcal{H}^{\left(e^{r^{\prime}}\right)} \subset \mathcal{H}$, labeled by integers $r^{\prime} \geqslant r$, such that:

(1) For each $r^{\prime} \geqslant r$, we have $\mathcal{H}^{\left(\ell^{r^{\prime}}\right)}=\mathcal{H}^{\ell, p} \mathcal{U}_{\ell}\left(\ell^{r^{\prime}}\right) \mathcal{H}_{p}$, where $\mathcal{H}^{\ell, p}$ and $\mathcal{H}_{p}$ are as above, and where $\mathcal{U}_{\ell}\left(\ell^{r^{\prime}}\right):=\operatorname{ker}\left(\mathrm{G}\left(\mathbb{Z}_{\ell}\right) \rightarrow \mathrm{G}\left(\mathbb{Z} / \ell^{r^{\prime}} \mathbb{Z}\right)\right)$.

(2) By writing $\mathcal{H}^{\prime}=\mathcal{H}^{\left(\ell^{r^{\prime}}\right)}$ for simplicity, the corresponding canonical morphisms $\mathrm{X}_{\mathcal{H}^{\prime}} \rightarrow \mathrm{X}_{\mathcal{H}}$ and their boundary analogues $\Xi^{\prime} \rightarrow \Xi, C^{\prime} \rightarrow C$ and $\mathbf{Z}^{\prime} \rightarrow \mathbf{Z}$ are finite étale. The morphisms $C \rightarrow \mathbf{Z}$ and $C^{\prime} \rightarrow \mathbf{Z}^{\prime}$ are abelian scheme torsors over finite étale covers of the base schemes, and the morphisms $\Xi \rightarrow C$ and $\Xi^{\prime} \rightarrow C^{\prime}$ are torus torsors. The induced morphism $C^{\prime} \rightarrow C \times{ }_{\mathrm{Z}} \mathrm{Z}^{\prime}$ is étale locally over $\mathrm{Z}^{\prime}$ the multiplication by $\ell^{r^{\prime}-r}$ on an 
abelian scheme, and the canonical morphism $\Xi^{\prime} \rightarrow \Xi \times{ }_{C} C^{\prime}$ is Zariski locally over $C^{\prime}$ the multiplication by $\ell^{r^{\prime}-r}$ on a torus.

Proof. Part (1) of the lemma is just the definition of $\mathcal{H}^{\left(\ell^{r^{\prime}}\right)} \subset \mathcal{H}$, for each $r^{\prime} \geqslant r$. It remains to verify the assertions in part (2) of the lemma. In Case (Sm), these follow from the constructions in [36, Sections 6.2.2-6.2.4; see also the errata]. In Cases $(\mathrm{Nm})$ and $(\mathrm{Spl})$, since $\mathcal{H} / \mathcal{H}^{\prime} \cong \mathcal{U}_{\ell}\left(\ell^{r}\right) / \mathcal{U}_{\ell}\left(\ell^{r^{\prime}}\right)$, we can define relative moduli $C^{\prime \text {,mod }}$ and $\Xi^{\prime \text {,mod }}$ over $C$ and $\Xi$ parameterizing the $\ell$-power parts of the degenerations of level structures, as in [36, Section 6.2.4] (see also [42, Section 1.3.2]), which are finite étale over $C$ and $\Xi$, respectively, and have the desired property as (instead of $C^{\prime}$ and $\Xi^{\prime}$ ) in the last sentence of the lemma. Then the induced morphisms $C^{\prime} \rightarrow C^{\prime \text {,mod }}$ and $\Xi^{\prime} \rightarrow \Xi^{\prime \text {,mod }}$ are isomorphisms by Zariski's main theorem, because they are finite morphisms between normal schemes which induce isomorphisms in characteristic zero. In Case (Hdg), these follow from the boundary descriptions in characteristic zero in [50, Section 2.1] (based on [4] and [71]) and in characteristic $p$ in [50, Sections 4.1-4.2].

\section{Let $Z$ be a stratum of $X_{\mathcal{H}}^{\min }$.}

In Cases $(\mathrm{Sm}),(\mathrm{Nm})$, or $(\mathrm{Spl})$, the stratum $Z$ is associated with some cusp label $\left[\left(Z_{\mathcal{H}}, \Phi_{\mathcal{H}}, \delta_{\mathcal{H}}\right)\right]$, which determines an $\mathcal{H}$-orbit $Z_{\mathcal{H}}$ of a fully symplectic liftable filtration $Z=\left\{Z_{-i}\right\}_{i \in \mathbb{Z}}$ on $L \otimes_{\mathbb{Z}} \hat{\mathbb{Z}}$ (see [36, Definitions 5.2.7.1 and 5.4.2.4]). Then we fix the choices of $P$ and $V=\left\{V_{-i}\right\}_{i \in \mathbb{Z}}$ as in Lemma 3.3.6, where $V_{-2} \otimes_{\mathbb{Q}} \mathbb{A}^{\infty}$ lies in the $\mathcal{H}$-orbit of $Z_{-2} \otimes_{\mathbb{Z}} \mathbb{Q}$, and define various groups as in Definition 3.3.8.

In Case (Hdg), it is the consequence of the characteristic zero theory in [5] and [71] that the stratum $Z$ is associated with some parabolic subgroup $P$ of $G \otimes_{\mathbb{Z}}$ $\mathbb{Q}$ whose image in each simple factor of $\left(G \otimes_{\mathbb{Z}} \mathbb{Q}\right)^{\text {ad }}$ is either the whole factor or a proper maximal parabolic subgroup. Let $\mathrm{U}$ and $\mathrm{M}$ denote the unipotent radical and the Levi quotient of $\mathrm{P}$, respectively. Then we have an exact sequence $1 \rightarrow \mathrm{G}_{h} \rightarrow$ $\mathrm{M} \rightarrow \mathrm{G}_{l} \rightarrow 1$, where $\mathrm{G}_{h}$ defines the boundary analogue of $\mathrm{X}_{\mathcal{H}}$, which is a finite cover of $Z$ in [71] and [50]. (Unlike in PEL cases, we do not have $M \cong G_{l} \times G_{h}$ in general.) In what follows, we will not be as precise as in Cases (Sm), (Nm), and (Spl), but only state the bare minimum for the application of Pink's arguments in [72].

REMARK 4.3.3. The group $\Gamma$ in Proposition 2.1.2 is by definition a subgroup of $\mathrm{G}_{l}(\mathbb{Q})$. Even in Cases $(\mathrm{Sm}),(\mathrm{Nm})$, or $(\mathrm{Spl})$, where $\mathrm{M} \cong \mathrm{G}_{l} \times \mathrm{G}_{h}$, it is generally not true that $\mathcal{H}_{\mathrm{M}}$ coincides with the semidirect product $\mathcal{H}_{l} \rtimes \mathcal{H}_{h}$ or $\mathcal{H}_{l}^{\prime} \ltimes \mathcal{H}_{h}^{\prime}$ (see [36, Definitions 5.3.1.4 and 5.3.1.1, and the errata], and see [42, Definitions 1.2.1.11 and 1.2.1.12]), and $\Gamma^{\prime}:=\Gamma \cap \mathcal{H}_{l}$ is a finite index subgroup of $\Gamma$ which is generally different from $\Gamma$. This discrepancy between $\Gamma$ and $\Gamma^{\prime}$ defines a finite étale cover of $Z$ in characteristic zero, which is consistent with the consideration in 
[71, Section 6.3] (cf. Definition 4.3.4 below). Nevertheless, in Cases (Sm), (Nm), or $(\mathrm{Spl}), \Gamma^{\prime}=\Gamma$ when and exactly when $\mathrm{M} \cong \mathrm{G}_{l} \times \mathrm{G}_{h}$ induces a direct product $\mathcal{H}_{\mathrm{M}} \cong \mathcal{H}_{l} \times \mathcal{H}_{h}$, in which case $\mathcal{H}_{l}=\mathcal{H}_{l}^{\prime}$ and $\mathcal{H}_{h}^{\prime}=\mathcal{H}_{h, \Phi}=\mathcal{H}_{h}$. This is the case, for example, when $\mathcal{H}$ is a principal level (and when the technical assumption [36, Condition 1.4.3.10] is satisfied).

Consider any algebraic representation $\xi$ of $G \otimes_{\mathbb{Z}} \mathbb{Q}$ on a finite-dimensional vector space $V_{\xi}$ over $\overline{\mathbb{Q}}_{\ell}$, with an associated étale sheaf $\mathcal{V}_{\xi}$ over $\mathrm{X}_{\mathcal{H}}$, as explained in [45, Section 3.1] (which is consistent with [72, Sections 1 and 4.9]). The association is functorial and also applies to complexes $V$ of algebraic representations of $G \otimes_{\mathbb{Z}} \mathbb{Q}$ on finite-dimensional vector spaces over $\overline{\mathbb{Q}}_{\ell}$, with associated complexes $\mathcal{V}$ of $\overline{\mathbb{Q}}_{\ell}$-étale sheaves over $\mathbf{X}_{\mathcal{H}}$. For each algebraic group $(\cdot)$ over $\mathbb{Q}$, let $D^{b}\left(\cdot, \overline{\mathbb{Q}}_{\ell}\right)$ denote the bounded derived category of algebraic representations of $(\cdot)$ on finite-dimensional vector spaces over $\overline{\mathbb{Q}}_{\ell}$. Then the above association induces a functor from $D^{b}\left(\mathrm{G} \otimes_{\mathbb{Z}} \mathbb{Q}, \overline{\mathbb{Q}}_{\ell}\right)$ to $D_{c}^{b}\left(\mathrm{X}_{\mathcal{H}}, \overline{\mathbb{Q}}_{\ell}\right)$ (see the beginning of Section 4.1).

Following [72, Section 5.2], let us introduce the following definitions:

Definition 4.3.4. For the group $\Gamma$ in Proposition 2.1.2 associated with Z, consider the Stein factorization

$$
C^{\text {st }}:=\underline{\operatorname{Spec}}_{\mathscr{O}_{\mathrm{Z}}}\left((C \rightarrow \mathrm{Z})_{*} \mathscr{O}_{C}\right) \rightarrow \mathrm{Z},
$$

with an induced action of $\Gamma$. Then we denote by $\Gamma^{\prime}$ the largest (finite index) subgroup of $\Gamma$ which acts trivially on $C^{\text {st }}$. (In Cases (Sm), (Nm), and (Spl), this is the same $\Gamma^{\prime}$ as in Remark 4.3.3.)

Definition 4.3.5. Let $\mathfrak{u}:=\operatorname{Lie} U\left(\mathbb{Q}_{\ell}\right)$ denote the Lie algebra of the $\ell$-adic analytic Lie group $\mathrm{U}\left(\mathbb{Q}_{\ell}\right)$. For each $V \in D^{b}\left(\mathrm{P}, \overline{\mathbb{Q}}_{\ell}\right)$, we denote by $R \operatorname{Inv}(\mathfrak{u}, V)$ the object of $D^{b}\left(\mathrm{M}, \overline{\mathbb{Q}}_{\ell}\right)$ whose cohomology computes the Lie algebra cohomology of $V$.

Definition 4.3.6. For each object $V \in D^{b}\left(\mathrm{M}, \overline{\mathbb{Q}}_{\ell}\right)$, we denote by $R \operatorname{Inv}\left(\Gamma^{\prime}, V\right)$ the object of $D^{b}\left(\mathrm{G}_{h}, \overline{\mathbb{Q}}_{\ell}\right)$ whose cohomology computes the group cohomology of $V$.

REMARK 4.3.7. In Cases $(\mathrm{Sm}),(\mathrm{Nm})$, or $(\mathrm{Spl})$, the boundary stratum $\mathrm{Z}$ and its finite cover $C^{\text {st }}$ are analogues of $\mathrm{X}_{\mathcal{H}}$ associated with $\mathrm{G}_{h}$ at levels $\mathcal{H}_{h, \Phi}$ and $\mathcal{H}_{h}^{\prime}$, respectively, and we have $C^{\text {st }} /\left(\Gamma / \Gamma^{\prime}\right) \stackrel{\sim}{\rightarrow} \mathrm{Z}$. In Case (Hdg), it is only the $C^{\text {st }}$ in Definition 4.3.4 that is associated with $\mathrm{G}_{h}$, but we still have $C^{\text {st }} /\left(\Gamma / \Gamma^{\prime}\right) \stackrel{\sim}{\rightarrow} \mathrm{Z}$. In all cases, the constructions in [45, Section 3.1] and [72, Sections 1 and 4.9] associate an object of $D_{c}^{b}\left(C^{\text {st }}, \overline{\mathbb{Q}}_{\ell}\right)$ with any $R \operatorname{Inv}\left(\Gamma^{\prime}, V\right)$ as in Definition 4.3.6, 
which admits an action of $\Gamma / \Gamma^{\prime}$ and defines an object of $D_{c}^{b}\left(\mathrm{Z}, \overline{\mathbb{Q}}_{\ell}\right)$ (cf. [71, (1.10) and (4.7)]) because $C^{\text {st }} \rightarrow Z$ is étale by assumption (see (4) of Assumption 4.3.1).

Definition 4.3.8. For each object $V \in D^{b}\left(\mathrm{G} \otimes_{\mathbb{Z}} \mathbb{Q}, \overline{\mathbb{Q}}_{\ell}\right)$, we define

$$
V_{\mathrm{Z}}^{\natural}:=R \operatorname{Inv}\left(\Gamma^{\prime}, R \operatorname{Inv}\left(\mathfrak{u},\left.V\right|_{\mathrm{P}}\right)\right)
$$

as in Definition 4.3.6, with an associated object $\mathcal{V}_{Z}^{\natural}$ of $D_{c}^{b}\left(Z, \overline{\mathbb{Q}}_{\ell}\right)$ as in Remark 4.3.7.

When $V$ is represented by an algebraic representation $V_{\xi}$ of $\mathrm{G} \otimes_{\mathbb{Z}} \mathbb{Q}$ on a finitedimensional vector space over $\overline{\mathbb{Q}}_{\ell}$, we denote by $V_{\xi, Z}^{\natural}$ the unique representative of $V_{Z}^{\natural}$ which is a direct sum of shifts of algebraic representations of $\mathrm{G}_{h}$ on finitedimensional vector spaces over $\overline{\mathbb{Q}}_{\ell}$ (which exists because $\mathrm{G}_{h}$ is reductive), and by $\mathcal{V}_{\xi, Z}^{\natural}$ the associated direct sum of shifts of $\overline{\mathbb{Q}}_{\ell}$-étale sheaves over $Z$ representing $\mathcal{V}_{Z}^{\natural}$. As usual, we shall also denote by $V_{\xi}, \mathcal{V}_{\xi}, V_{\xi, Z}^{\natural}$, and $\mathcal{V}_{\xi, Z}^{\natural}$ the induced objects in the derived categories.

Suppose $\mathrm{T}$ is just a point. Let $i^{\mathrm{min}}: \mathrm{Z}_{\mathrm{T}} \rightarrow\left(\mathrm{X}_{\mathcal{H}}^{\min }\right)_{\mathrm{T}}$ and $i^{\text {tor }}: \tilde{Z}_{\mathrm{T}} \rightarrow\left(\mathrm{X}_{\mathcal{H}, \Sigma}^{\text {tor }}\right)_{\mathrm{T}}$ (respectively $j^{\text {min }}:\left(\mathbf{X}_{\mathcal{H}}\right)_{\top} \hookrightarrow\left(\mathrm{X}_{\mathcal{H}}^{\min }\right)_{\mathrm{T}}$ and $\left.j^{\text {tor }}:\left(\mathrm{X}_{\mathcal{H}}\right)_{\mathrm{T}} \hookrightarrow\left(\mathrm{X}_{\mathcal{H}, \Sigma}^{\text {tor }}\right)_{\mathrm{T}}\right)$ denote the canonical locally closed (respectively open) immersions, which satisfy $i^{\text {min }}=$ $\oint \circ i^{\text {tor }}$ and $j^{\min }=\oint \circ j^{\text {tor }}$, where $\oint:=\left(\oint_{\mathcal{H}, \Sigma}\right)_{\mathrm{T}}:\left(\mathbf{X}_{\mathcal{H}, \Sigma}^{\text {tor }}\right)_{\mathrm{T}} \rightarrow\left(\mathbf{X}_{\mathcal{H}}^{\min }\right)_{\mathrm{T}}$ is as in (1) of Theorem 2.3.2.

THEOREM 4.3.10 (Pink). Let $V_{\xi}$ be an algebraic representation of $\mathrm{G} \otimes_{\mathbb{Z}} \mathbb{Q}$ on a finite-dimensional vector space over $\overline{\mathbb{Q}}_{\ell}$, which defines $\mathcal{V}_{\xi}$, and so on, as in Definition 4.3.8. For simplicity, we shall denote by the same symbols the pullbacks of $\mathcal{V}_{\xi}$ and $\mathcal{V}_{\xi, Z}^{\natural}$ under $\mathrm{T} \rightarrow \mathrm{S}$. Then, under Assumption 4.3.1, there exists a canonical isomorphism

$$
i^{\min , *} R j_{*}^{\min } \mathcal{V}_{\xi} \stackrel{\sim}{\rightarrow} \mathcal{V}_{\xi, Z}^{\natural}
$$

in $D_{c}^{b}\left(\mathrm{Z}_{\mathrm{T}}, \overline{\mathbb{Q}}_{\ell}\right)$. More precisely, by the proper base change theorem (see [3, XII, 5.1]), we have a canonical isomorphism

$$
i^{\min , *} R j_{*}^{\min } \mathcal{V}_{\xi} \stackrel{\sim}{\rightarrow} R \oint_{\mathrm{Z}, *} i^{\mathrm{tor}, *} R j_{*}^{\mathrm{tor}} \mathcal{V}_{\xi}
$$

where $\oint_{\mathrm{Z}}: \tilde{Z}_{\mathrm{T}} \rightarrow \mathrm{Z}_{\mathrm{T}}$ is the pullback of $\oint$. Then the upshot is the existence of an isomorphism 


$$
R \oint_{\mathrm{Z}, *} i^{\mathrm{tor}, *} R j_{*}^{\mathrm{tor}} \mathcal{V}_{\xi} \stackrel{\sim}{\rightarrow} \mathcal{V}_{\xi, \mathrm{Z}}^{\natural},
$$

which depends only on $\left.V_{\xi}\right|_{\mathrm{P}}$, such that (4.3.11) is the composition

$$
i^{\min , *} R j_{*}^{\min } \mathcal{V}_{\xi} \stackrel{(4.3 .12)}{\rightarrow} R \oint_{\mathrm{Z}, *} i^{\text {tor, } *} R j_{*}^{\text {tor }} \mathcal{V}_{\xi} \stackrel{(4.3 .13)}{\rightarrow} \mathcal{V}_{\xi, \mathrm{Z}}^{\natural}
$$

As level varies, such isomorphisms are compatible with Hecke actions of elements of $\mathrm{P}\left(\mathbb{A}^{\infty}\right)$ (when defined). (In characteristic $p$, we usually cannot expect the Hecke actions to be defined for all elements of $\mathrm{P}\left(\mathbb{A}^{\infty}\right)$.)

Proof. The existence of the canonical isomorphism (4.3.11) follows from [72, Theorem 4.2.1 and Proposition 5.2.1], while the later explanatory statements are based on the proofs there. (We could have referred to [72, Theorem 5.3.1] when the simplifying assumption $H_{Q}=H_{C} K_{P}$ there is satisfied, which corresponds to the assumption that $\Gamma=\Gamma^{\prime}$ in our setting; cf. [72, paragraph after (3.7.4)], Remark 4.3.3, and Definition 4.3.4.) Although they were stated only over $\operatorname{Spec}\left(F_{0}\right)$, the same arguments there work as long as there are systems of minimal and toroidal compactifications with the same axiomatic properties as in [72, Sections 3.7-3.11; see also Section 4.9], which is the case here, by considering higher principal levels at $\ell$, thanks to Lemma 4.3.2.

REMARK 4.3.15. The proof of Theorem 4.3.10 in [72] is consistent with the one here: In Cases $(\mathrm{Sm}),(\mathrm{Nm})$, and $(\mathrm{Spl})$, this is because of the comparisons in [34, Theorems 4.1.1 and 5.1.1; see also the errata] and in [37]. In Case (Hdg), this is because [50] and [72] were based on the same characteristic zero results in [71].

Let $Y$ be a well-positioned subset of $\left(X_{\mathcal{H}}\right)_{T}$, with associated $Y^{\natural}=\left\{Y_{Z}^{\natural}\right\}_{Z}$ as in Definition 2.2.1. Let $Y^{\min }$ and $Y_{\Sigma}^{\text {tor }}$ be its partial minimal and toroidal compactifications, as in Definition 2.3.1, with the canonical proper surjection $\oint_{Y}:=\oint_{Y, \Sigma}: Y_{\Sigma}^{\text {tor }} \rightarrow Y^{\text {min }}$, as in Theorem 2.3.2. Let $i_{Y}^{\min }: Y_{Z} \rightarrow Y^{\text {min }}$ and $i_{Y}^{\text {tor }}:$ $\mathrm{Y}_{\tilde{Z}} \rightarrow \mathrm{Y}_{\Sigma}^{\text {tor }}$ (respectively $j_{\mathrm{Y}}^{\text {min }}: \mathrm{Y} \hookrightarrow \mathrm{Y}^{\text {min }}$ and $j_{\mathrm{Y}}^{\text {tor }}: \mathrm{Y} \hookrightarrow \mathrm{Y}_{\Sigma}^{\text {tor }}$ ) denote the canonical locally closed (respectively open) immersions, which satisfy $i_{Y}^{\min }=\oint_{Y} \circ i_{Y}^{\text {tor }}$ and $j_{Y}^{\min }=\oint_{Y} \circ j^{\text {tor }}$. Now we can formulate our generalization of Theorem 4.3.10:

THEOREM 4.3.16. Let $\mathcal{F}$ be a well-positioned complex over $\mathrm{Y}_{\Sigma}^{\text {tor }}$, equipped with a collection $\mathcal{F}^{\natural}=\left\{\left(\mathcal{F}_{\mathrm{Z}}^{\natural}, \iota_{\mathrm{Z}}\right)\right\}_{\mathrm{Z}}$ as in Definition 4.1.3. Let $V_{\xi}$ be an algebraic representation of $\mathrm{G} \otimes_{\mathbb{Z}} \mathbb{Q}$ on a finite-dimensional vector space over $\overline{\mathbb{Q}}_{\ell}$. Let $\mathcal{F}_{Y}$, $\mathcal{V}_{\xi, Y}$, and $\mathcal{V}_{\xi, Y_{Z}}^{\natural}$ denote the pullbacks of $\mathcal{F}, \mathcal{V}_{\xi}$, and $\mathcal{V}_{\xi, Z}^{\natural}$ (see Definition 4.3.8) to $\mathrm{Y}$, $\mathrm{Y}$, and $\mathrm{Y}_{\mathrm{Z}}$, respectively. Then, under Assumption 4.3.1, there exists a canonical 
isomorphism

$$
i_{\mathrm{Y}}^{\min , *} R j_{\mathrm{Y}, *}^{\min }\left(\mathcal{V}_{\xi, \mathrm{Y}} \otimes^{L} \mathcal{F}_{\mathrm{Y}}\right) \stackrel{\sim}{\rightarrow} \mathcal{V}_{\xi, Y_{\mathrm{Z}}}^{\natural} \otimes^{L} \mathcal{F}_{\mathrm{Z}}^{\natural}
$$

in $D_{c}^{b}\left(\mathrm{Y}_{\mathrm{Z}}, \overline{\mathbb{Q}}_{\ell}\right)$. As level varies, such isomorphisms are compatible with actions of elements of $\mathrm{P}\left(\mathbb{A}^{\infty}\right)$ when they are defined on some collections of $\mathrm{Y}$ and $\mathcal{F}_{Z}^{\natural}$ (which is possible by Lemma 4.1.12).

In order to prove Theorem 4.3.16, we need some further preparations.

LEMMA 4.3.18. There is a canonical isomorphism

$$
i_{\mathrm{Y}}^{\min , *} R j_{\mathrm{Y}, *}^{\min }\left(\mathcal{V}_{\xi, \mathrm{Y}} \otimes^{L} \mathcal{F}_{\mathrm{Y}}\right) \stackrel{\sim}{\rightarrow} R \oint_{\mathrm{Y}_{\mathrm{Z}, *}} i_{\mathrm{Y}}^{\mathrm{tor}, *} R j_{\mathrm{Y}, *}^{\mathrm{tor}}\left(\mathcal{V}_{\xi, \mathrm{Y}} \otimes^{L} \mathcal{F}_{\mathrm{Y}}\right),
$$

where $\oint_{Y_{Z}}: Y_{\tilde{Z}} \rightarrow Y_{Z}$ is the pullback of $\oint_{Y}$.

Proof. This follows from the proper base change theorem (see [3, XII, 5.1]).

LEMMA 4.3.20. Let $\Lambda$ be as in the beginning of Section 4.1. Suppose $f: T \rightarrow T^{\prime}$ is a morphism between schemes of residue characteristics prime to $\ell$, which we assume to be separated and of finite type over some regular scheme of dimension $\leqslant 1$. Suppose $\mathcal{G} \in D_{c}^{b}(T, \Lambda)$ and $\mathcal{H} \in D_{c}^{b}\left(T^{\prime}, \Lambda\right)$. Then there is a canonical morphism

$$
\left(R f_{*} \mathcal{G}\right) \otimes^{L} \mathcal{H} \rightarrow R f_{*}\left(\mathcal{G} \otimes^{L}\left(f^{*} \mathcal{H}\right)\right)
$$

in $D_{c}^{b}\left(T^{\prime}, \Lambda\right)$.

Proof. By adjunction, we have a canonical morphism $f^{*} R f_{*} \mathcal{G} \rightarrow \mathcal{G}$, which induces a canonical morphism

$$
f^{*}\left(\left(R f_{*} \mathcal{G}\right) \otimes^{L} \mathcal{H}\right) \stackrel{\sim}{\rightarrow}\left(f^{*} R f_{*} \mathcal{G}\right) \otimes^{L}\left(f^{*} \mathcal{H}\right) \rightarrow \mathcal{G} \otimes^{L}\left(f^{*} \mathcal{H}\right),
$$

which in turn induces the desired morphism (4.3.21).

Consequently, we have a canonical morphism

$$
\left(R j_{\mathrm{Y}, *}^{\text {tor }} \mathcal{V}_{\xi, \mathrm{Y}}\right) \otimes^{L} \mathcal{F} \rightarrow R j_{\mathrm{Y}, *}^{\text {tor }}\left(\mathcal{V}_{\xi, \mathrm{Y}} \otimes^{L} \mathcal{F}_{\mathrm{Y}}\right)
$$

LEMMA 4.3.23. In Lemma 4.3.20, suppose that $f: T \rightarrow T^{\prime}$ is a morphism between schemes of finite type over $S_{0}=\operatorname{Spec}(k)$ for some field $k$, which is étale locally of the form $f=g \times{ }_{S_{0}} \operatorname{Id}_{S}: T=E \times \times_{S_{0}} S \hookrightarrow T^{\prime}=E^{\prime} \times_{S_{0}} S$, where $g: E \rightarrow E^{\prime}$ is a morphism between schemes separated and of finite type over $S_{0}$, and where $S$ is of finite type over $S_{0}$. Suppose moreover that such an 
étale local factorization of $f$ can be chosen such that $\mathcal{G}$ and $\mathcal{H}$ are the pullbacks of some $\mathcal{L} \in D_{c}^{b}(E, \Lambda)$ and $\mathcal{M} \in D_{c}^{b}(S, \Lambda)$, respectively. Then (4.3.21) is an isomorphism.

Proof. Up to étale localization, we may identify the morphism (4.3.21) with the Künneth morphism $\left(R g_{*} \mathcal{L}\right) \bigotimes_{S_{0}}^{L} \mathcal{M} \rightarrow R f_{*}\left(\mathcal{L} \otimes_{S_{0}}^{L} \mathcal{M}\right)$, which is an isomorphism by $[6,4.2 .7]$.

REMARK 4.3.24. In Lemma 4.3.20, when $f$ is compactifiable, we have a canonical isomorphism $(R f: \mathcal{G}) \otimes^{L} \mathcal{H} \stackrel{\sim}{\rightarrow} R f_{!}\left(\mathcal{G} \otimes^{L}\left(f^{*} \mathcal{H}\right)\right)$, which is the wellknown projection formula (see [3, XVII, 5.2.9]), by the proper base change theorem (see [3, XVII, 5.2.2]). However, such a formula is false in general for $R f_{*}$ instead of $R f_{!}$.

COROLlARY 4.3.25. The canonical morphism (4.3.22) is an isomorphism.

Proof. For each Z, let $\mathcal{U}_{Z}=\left\{\left(\bar{U}_{i}, a_{\bar{U}_{i}}, a_{\bar{U}_{i}, E\left(\sigma_{i}\right)}^{\natural}\right)\right\}_{i \in I_{Z}}$ be as in Definition 4.1.3. Let $U_{i}$ denote the common preimage of $\mathrm{X}_{\mathcal{H}}$ and $E$ in $\bar{U}_{i}$ (see Corollary 2.1.7), and let $a_{\mathrm{Y}_{U_{i}}}: \mathrm{Y}_{U_{i}}:=U_{i} \times \mathrm{X}_{\mathcal{H}} \mathrm{Y} \rightarrow \mathrm{Y}, a_{\bar{U}_{\bar{U}_{i}}}: \mathrm{Y}_{\bar{U}_{i}}:=\bar{U}_{i} \times_{\chi_{\mathcal{H}, \Sigma}^{\text {tor }}} \mathrm{Y}_{\Sigma}^{\text {tor }} \rightarrow \mathrm{Y}_{\Sigma}^{\text {tor }}, J_{\bar{U}_{i}}: \mathrm{Y}_{U_{i}} \hookrightarrow \mathrm{Y}_{\bar{U}_{i}}$, and $a_{Y_{U_{i}}}^{\natural}: Y_{U_{i}} \rightarrow Y_{Z}^{\natural}$ denote the induced canonical morphisms. In order to show that (4.3.22) is an isomorphism, by étale localization, it suffices to show that, for each $Z$ and each $i \in I_{Z}$ as above, the induced morphism

$$
\left(R J_{\bar{U}_{i}, *} a_{\mathrm{Y}_{U_{i}}}^{*} \mathcal{V}_{\xi, Y}\right) \otimes^{L}\left(a_{\mathrm{Y}_{\bar{U}_{i}}}^{*} \mathcal{F}\right) \rightarrow R J_{\bar{U}_{i}, *} a_{\mathrm{Y}_{U_{i}}}^{*}\left(\mathcal{V}_{\xi, \mathrm{Y}} \otimes^{L} \mathcal{F}_{\mathrm{Y}}\right)
$$

is an isomorphism, for each $i \in I$. Since $\mathcal{V}_{\xi}$ is lisse, by using the isomorphism $\iota_{\bar{U}_{i}}$ : $a_{\mathrm{Y}_{\bar{U}_{i}}}^{*} \mathcal{F} \stackrel{\sim}{\rightarrow} a_{\mathrm{Y}_{\bar{U}_{i}}^{\natural}, *}^{\natural} \mathcal{F}_{Z}^{\natural}$ as in (4.1.5), this follows from Lemma 4.3.23, as desired.

LEMMA 4.3.27. It suffices to prove Theorem 4.3.16 when $\mathrm{Y}=\left(\mathrm{X}_{\mathcal{H}}\right)_{\mathrm{T}}$.

Proof. Let $J: \mathrm{Y} \rightarrow\left(\mathrm{X}_{\mathcal{H}}\right)_{\mathrm{T}}$ and $J_{\mathrm{Z}}: \mathrm{Y}_{\mathrm{Z}} \rightarrow \mathrm{Z}_{\mathrm{T}}$ denote the canonical morphisms. By [3, XVII, 5.1.2 and 5.1.6], we have

$$
J_{Z, !} i_{Y}^{\min , *} R j_{Y, *}^{\min }\left(\mathcal{V}_{\xi, Y} \otimes^{L} \mathcal{F}_{Y}\right) \cong i^{\min , *} R j_{*}^{\min }\left(J_{!}\left(\mathcal{V}_{\xi, Y} \otimes^{L} \mathcal{F}_{Y}\right)\right)
$$

Since $J_{!}\left(\mathcal{V}_{\xi, Y} \otimes^{L} \mathcal{F}_{Y}\right) \cong \mathcal{V}_{\xi} \otimes^{L}\left(J_{!} \mathcal{F}_{Y}\right)$ and $J_{Z, !}\left(\mathcal{V}_{\xi, Y_{Z}}^{\natural} \otimes^{L} \mathcal{F}_{Z}^{\natural}\right) \cong \mathcal{V}_{\xi, Z}^{\natural} \otimes^{L}\left(J_{Z, !} \mathcal{F}_{Z}^{\natural}\right)$ because $\mathcal{V}_{\xi}$ and $\mathcal{V}_{\xi, Z}^{\natural}$ are direct sums of shifted lisse sheaves, we are reduced to constructing (4.3.17) when $\mathrm{Y}, \mathcal{F}$, and $\mathcal{F}_{Z}^{\natural}$ are replaced with $\left(\mathrm{X}_{\mathcal{H}}\right)_{\mathrm{T}}, J_{!} \mathcal{F}$, and $J_{Z, !} \mathcal{F}_{Z}^{\natural}$, respectively. Hence, this lemma follows from (1) of Lemma 4.2.1. 
Proof of Theorem 4.3.16. By Lemma 4.3.27, we may and we shall assume that $\mathrm{Y}=\left(\mathrm{X}_{\mathcal{H}}\right)_{\mathrm{T}}$. In this case, the desired (4.3.17) is the following composition

$$
\begin{aligned}
& i_{\mathrm{Y}}^{\min , *} R j_{\mathrm{Y}, *}^{\min }\left(\mathcal{V}_{\xi, \mathrm{Y}} \otimes^{L} \mathcal{F}_{\mathrm{Y}}\right) \quad \stackrel{(4.3 .19)}{\rightarrow} \quad R \oint_{\mathrm{Y}_{\mathrm{Z}, *}} i_{\mathrm{Y}}^{\text {tor, }, *} R j_{\mathrm{Y}, *}^{\text {tor }}\left(\mathcal{V}_{\xi, \mathrm{Y}} \otimes^{L} \mathcal{F}_{\mathrm{Y}}\right) \\
& R \oint_{\mathrm{Y}_{\mathrm{Z}, *}} i_{\mathrm{Y}}^{\mathrm{tor}, *}(4.3 .22)^{-1} \\
& \stackrel{\sim}{\rightarrow} \quad R \oint_{\mathrm{Y}_{\mathrm{Z}, *}} i_{\mathrm{Y}}^{\mathrm{tor}, *}\left(\left(R j_{\mathrm{Y}, *}^{\mathrm{tor}} \mathcal{V}_{\xi, \mathrm{Y}}\right) \otimes{ }^{L} \mathcal{F}\right) \\
& \stackrel{\text { can. }}{\rightarrow} \quad R \oint_{\mathrm{Y}_{\mathrm{Z}, *}}\left(\left(i_{\mathrm{Y}}^{\mathrm{tor}, *} R j_{\mathrm{Y}, *}^{\mathrm{tor}} \mathcal{V}_{\xi, \mathrm{Y}}\right) \otimes^{L}\left(i_{\mathrm{Y}}^{\mathrm{tor}, *} \mathcal{F}\right)\right)
\end{aligned}
$$

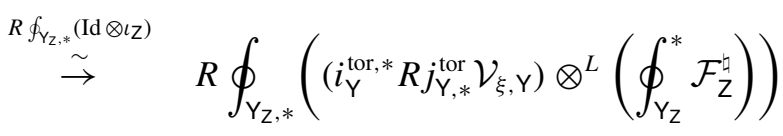

$$
\begin{aligned}
& \stackrel{\text { proj. }}{\rightarrow} \quad\left(R \oint_{\mathrm{Y}_{\mathrm{Z}, *}} i_{\mathrm{Y}}^{\text {tor,* }} R j_{\mathrm{Y}, *}^{\text {tor }} \mathcal{V}_{\xi, \mathrm{Y}}\right) \otimes^{L} \mathcal{F}_{\mathrm{Z}}^{\natural} \\
& \text { (4.3.13) } \\
& \stackrel{\sim}{\rightarrow} \quad \mathcal{V}_{\xi, Y_{Z}}^{\natural} \otimes^{L} \mathcal{F}_{Z}^{\natural},
\end{aligned}
$$

where (4.3.13) is applicable because $Y=\left(X_{\mathcal{H}}\right)_{T}$ and so $Y_{Z}^{\natural}=Z_{T}$; where (4.3.22) is an isomorphism by Corollary 4.3.25; and where the isomorphism denoted 'proj.' is based on the well-known projection formula (see [3, XVII, 5.2.9] and Remark 4.3.24), which is applicable because $\oint_{\mathrm{Y}_{\mathrm{Z}, *}}$ is proper.

REMARK 4.3.29. Instead of using Lemma 4.3.27 and (4.3.13), we could have directly established the isomorphism $R \oint_{\mathrm{Y}_{\mathrm{Z}, *}} i_{\mathrm{Y}}^{\text {tor, } *} R j_{\mathrm{Y}, *}^{\text {tor }} \mathcal{V}_{\xi, \mathrm{Y}} \stackrel{\sim}{\rightarrow} \mathcal{V}_{\xi, Y_{\mathrm{Z}}}^{\natural}$ needed in the last step of (4.3.28), without assuming $Y=\left(X_{\mathcal{H}}\right)_{\mathrm{T}}$, by the same argument as in the proof of Theorem 4.3.10, thanks to Theorem 2.3.2, Proposition 2.4.2, and Lemma 4.3.2.

REMARK 4.3.30. Theorems 4.3.10 and 4.3.16 have analogues over $\mathbb{Z}_{\ell}$-we then need to replace the Lie algebra cohomology in Definitions 4.3 .5 and 4.3 .8 by continuous group cohomology. (See [72, Theorem 4.2.1].)

REMARK 4.3.31. We have seen in Theorem 4.3.16 that $i_{Y}^{\min , *} R j_{\mathrm{Y}, *}^{\min }\left(\mathcal{V}_{\xi, Y} \otimes^{L}\right.$ $\mathcal{F}_{\mathrm{Y}}$ ), for any well-positioned complex $\mathcal{F}$, is canonically isomorphic to the (derived) tensor product of $\mathcal{V}_{\xi, Y_{Z}}^{\natural}$ and $\mathcal{F}_{Z}^{\natural}$, where $\mathcal{V}_{\xi, Y_{Z}}^{\natural}$ is the pullback of the complex $\mathcal{V}_{\xi, Z}^{\natural}$ essentially defined in [72]. This is intriguing because the minimal compactification is far from being a product near the boundary. As a consequence of this sheaf-theoretic product structure, we see that the following problems are, at least intuitively, unrelated: 
(1) Studying the bad reduction of a Shimura variety at $p$, or studying its nearby cycles, or studying the constant sheaves or intersection complexes on natural stratifications such as the Newton or Ekedahl-Oort ones.

(2) Studying the behavior of such complexes near the boundary strata of the minimal compactification.

REMARK 4.3.32. By Theorem 4.3.16, under Assumption 4.3.1, $\mathcal{V}_{\xi, Y_{Z}}^{\natural} \otimes^{L} \mathcal{F}_{Z}^{\natural}$ is uniquely determined by $\mathcal{F}$ and $V_{\xi}$, for an arbitrary $V_{\xi}$. When $V_{\xi}=\overline{\mathbb{Q}}_{\ell}$ is the trivial representation, $V_{\xi, Z}^{\natural}$ has a direct summand $\overline{\mathbb{Q}}_{\ell}$ in degree zero, by 'Lieberman's trick'. (This is a representation-theoretic statement independent of the consideration of integral models.) Therefore, $\mathcal{F}_{Z}^{\natural}$ is uniquely determined by $\mathcal{V}_{\xi, Y_{Z}}^{\natural} \otimes^{L} \mathcal{F}_{Z}^{\natural}$ in this case, and also by $\mathcal{F}$ (cf. Remark 4.1.10).

4.4. Mantovan's formula with boundary terms. The goal of this subsection is to present a generalization of [51, Section 8, Theorem 22] and [52, Theorem $3.1]$ in a way different from our previous one [45, Theorem 6.32], which also shows certain boundary terms. (See Theorem 4.4.7 and Remark 4.4.13 below.)

Let us resume the context of Section 3.3. Assume moreover that $p$ is a good prime for the integral PEL datum $\left(\mathcal{O}, \star, L,\langle\cdot, \cdot\rangle, h_{0}\right)$ as in [36, Definition 1.4.1.1]; that we are in Case $(\mathrm{Nm})$ with the trivial collection $\mathrm{J}=\left\{\mathrm{j}_{0}\right\}$ with $\left\{\left(g_{\mathrm{j}_{0}}\right.\right.$, $\left.\left.L_{\mathrm{j}_{0}},\langle\cdot, \cdot\rangle_{\mathrm{j}_{0}}\right)\right\}=\{(1, L,\langle\cdot, \cdot\rangle)\}$, as in [38, Example 2.3]; and that $\mathcal{O} \otimes_{\mathbb{Z}} \mathbb{Q}$ is simple and involves no factor of type $\mathrm{D}$, in the sense of [36, Definition 1.2.1.15]. (This is the setting in [45, Section 6.3].) In this case, $\left(G \otimes_{\mathbb{Z}} \mathbb{Q}\right)^{\text {ad }}$ is also simple over $\mathbb{Q}$. Let us also import Assumption 4.3.1 from Section 4.3. For the sake of concreteness, let us fulfil the requirement in Assumption 4.3.1 that $C \rightarrow \mathrm{Z}$ is an abelian scheme torsor for each $Z$ by assuming that $\mathcal{H}$ is a principal level, by Lemma 2.1.9. This will be harmless for our purpose in this subsection, because principal levels are cofinal among all choices of levels.

Suppose $\mathrm{S}=\operatorname{Spec}\left(\mathcal{O}_{K}\right)$, where $K$ is the $v$-adic completion of $F_{0}$ at a place $v \mid p$. Consider the nearby cycle functors $R \Psi_{\mathrm{X}_{\mathcal{H}}}$, and others, defined by some compatible choices of geometric points $\bar{\eta}=\operatorname{Spec}(\bar{K})$ and $\bar{s}$ above the generic and special points $\eta=\operatorname{Spec}(K)$ and $s$ of S, respectively, as in [45, Section 5.1]. For each $b \in \mathrm{B}\left(\mathrm{G} \otimes_{\mathbb{Z}} \mathbb{Q}_{p}\right)$, and for each irreducible algebraic representation $\xi$ of $\mathrm{G} \otimes_{\mathbb{Z}} \mathbb{Q}$ on a finite-dimensional vector space $V_{\xi}$ over $\overline{\mathbb{Q}}_{\ell}$, recall that Mantovan proved the following formula (see [45, Theorem 6.26], with $\mathcal{H}$ running over a cofinal system of higher levels, such as all higher principal levels)

$$
\sum_{i}(-1)^{i}\left[H_{\mathrm{et}, c}^{i}\left(\mathrm{X}_{\bar{s}}^{b},\left.\left(R \Psi_{\mathrm{X}}\left(\mathcal{V}_{\xi}\right)\right)\right|_{\mathrm{X}_{\bar{s}}^{b}}\right)\right]=\sum_{j}(-1)^{j} \mathcal{E}_{b}\left(\left[H_{\mathrm{et}, c}^{j}\left(\lg ^{b}, \mathcal{V}_{\xi}\right)\right]\right)
$$


between virtual representations of $\mathrm{G}\left(\mathbb{A}^{\infty, p}\right) \times \mathrm{G}\left(\mathbb{Q}_{p}\right)^{+} \times W_{K}$, where

$$
\mathrm{G}\left(\mathbb{Q}_{p}\right)^{+}:=\left\{g_{p} \in \mathrm{G}\left(\mathbb{Q}_{p}\right): g_{p}^{-1}\left(L \otimes_{\mathbb{Z}} \mathbb{Z}_{p}\right) \subset L \otimes_{\mathbb{Z}} \mathbb{Z}_{p}\right\}
$$

is a submonoid of $\mathrm{G}\left(\mathbb{Q}_{p}\right)$ (cf. [51, page 599]), where $\lg ^{b}$ is the limit of the socalled Igusa varieties over certain central leaf of $\mathrm{X}_{\bar{s}}^{b}$, and where $\mathcal{E}_{b}$ is the so-called Mantovan functor. (For more details, see [45, Section 6.3] and the references there to [51], [52], and other works.)

Let $Z$ be a stratum of $X_{\mathcal{H}}^{\min }$, which is associated with some parabolic subgroup $\mathrm{P}$ of $G \otimes_{\mathbb{Z}} \mathbb{Q}$, as in Lemma 3.3.6, with its various quotients of subgroups defined in Definition 3.3.8. Since $\mathcal{O} \otimes_{\mathbb{Z}} \mathbb{Q}$ is simple by assumption, P is either all of $G \otimes_{\mathbb{Z}} \mathbb{Q}$, or a proper maximal parabolic subgroup of $\mathrm{G} \otimes_{\mathbb{Z}} \mathbb{Q}$. For each $b \in \mathrm{B}\left(\mathrm{G} \otimes_{\mathbb{Z}} \mathbb{Q}_{p}\right)$, by Proposition 3.3.9 and its proof, $\mathrm{Y}:=\left(\mathrm{X}_{\mathcal{H}}\right)_{s}^{b}$ is a well-positioned subset of $\left(\mathrm{X}_{\mathcal{H}}\right)_{s}$, associated with the collection $\mathrm{Y}^{\natural}:=\left\{Y_{Z}^{\natural}:=Z_{s}^{b}\right\}_{Z}$, where $Z_{s}^{b}$ is either the empty subset or the Newton stratum $Z_{s}^{b_{h}}$ of $Z_{s}$. In the latter case, $\left(\mathbf{X}_{\mathcal{H}}\right)_{s}^{b} \neq \emptyset$, and $b$ is the image of some $b_{\mathrm{P}} \in \mathrm{B}\left(\mathrm{P} \otimes_{\mathbb{Q}} \mathbb{Q}_{p}\right)$, with image $b_{\mathrm{M}}=\left(b_{l}, b_{h}\right)$ in $\mathrm{B}\left(\mathrm{M} \otimes_{\mathbb{Q}}\right.$ $\left.\mathbb{Q}_{p}\right) \cong \mathrm{B}\left(\mathrm{G}_{l} \otimes_{\mathbb{Q}} \mathbb{Q}_{p}\right) \times \mathrm{B}\left(\mathrm{G}_{h} \otimes_{\mathbb{Q}} \mathbb{Q}_{p}\right)$. Conversely, by the construction of Mumford families, every $b_{h} \in \mathrm{B}\left(\mathrm{G}_{h} \otimes_{\mathbb{Q}} \mathbb{Q}_{p}\right)$ such that $Z_{s}^{b_{h}}$ is nonempty comes from some $b \in \mathrm{B}\left(\mathrm{G} \otimes_{\mathbb{Z}} \mathbb{Q}_{p}\right)$ as above. For such $\mathrm{Y}$ and $\mathrm{Y}^{\natural}$, let $\left(\mathrm{X}_{\mathcal{H}, \Sigma}^{\text {tor }}\right)_{s}^{b}:=\mathrm{Y}_{\Sigma}^{\text {tor }}$ be defined as in Definition 2.3.1.

By Lemmas 4.2.1 and 4.2.10, $\left.R \Psi_{\mathrm{X}_{\mathcal{H}, \Sigma}^{\text {tor }}}\left(\overline{\mathbb{Q}}_{\ell}\right)\right|_{\left(\mathrm{X}_{\mathcal{H}, \Sigma}^{\text {tor }}\right)_{s}^{\frac{b}{s}}}$ is a well-positioned complex over $\left(\mathrm{X}_{\mathcal{H}, \Sigma}^{\text {tor }}\right)_{\bar{s}}$, equipped with the collection $\left\{\left.R \Psi_{Z}\left(\overline{\mathbb{Q}}_{\ell}\right)\right|_{Z_{\bar{s}}^{b}}\right\}_{Z}$. By abuse of notation, we shall still denote their extensions by zero to the whole schemes $\left(X_{\mathcal{H}, \Sigma}^{\text {tor }}\right)_{\bar{s}}$ and $Z_{\bar{s}}$, respectively, by the same symbols. Therefore, by Theorem 4.3.16, we have an isomorphism

$$
i_{\bar{s}}^{\min , *} R j_{\bar{s}, *}^{\min }\left(\left.\left.\mathcal{V}_{\xi} \otimes^{L} R \Psi_{\mathrm{X}_{\mathcal{H}}}\left(\overline{\mathbb{Q}}_{\ell}\right)\right|_{\left(\mathrm{X}_{\mathcal{H}}\right)_{\bar{s}}^{b}} \stackrel{\sim}{\rightarrow} \mathcal{V}_{\xi, Z}^{\natural} \otimes^{L} R \Psi_{\mathrm{Z}}\left(\overline{\mathbb{Q}}_{\ell}\right)\right|_{Z_{\bar{s}}^{b}}\right.
$$

in $D_{c}^{b}\left(Z_{\bar{s}}, \overline{\mathbb{Q}}_{\ell}\right)$, where $\mathcal{V}_{\xi}$ and $\mathcal{V}_{\xi, Z}^{\natural}$ also denote their pullbacks to $\left(\mathbf{X}_{\mathcal{H}}\right)_{\bar{s}}$ and $Z_{\bar{s}}$, respectively. Note that (4.4.2) is not just an isomorphism in $D_{c}^{b}\left(Z_{\bar{s}}, \overline{\mathbb{Q}}_{\ell}\right)$, but also an isomorphism in $D_{c}^{b}\left(Z_{\bar{s}} \times \bar{\eta}, \overline{\mathbb{Q}}_{\ell}\right)$, because the proof of Theorem 4.3.16 only uses canonical isomorphisms which are compatible with the actions of $\operatorname{Gal}(k(\bar{\eta}) / k(\eta))=\operatorname{Gal}(\bar{K} / K)$ (cf. Lemma 4.2.10 and its proof).

Since $\mathcal{V}_{\xi}$ is lisse, the canonical morphism $\mathcal{V}_{\xi} \otimes^{L} R \Psi_{\mathrm{X}_{\mathcal{H}}}\left(\overline{\mathbb{Q}}_{\ell}\right) \rightarrow R \Psi_{\mathrm{X}_{\mathcal{H}}}\left(\mathcal{V}_{\xi}\right)$ is an isomorphism in $D_{c}^{b}\left(\left(\mathbf{X}_{\mathcal{H}}\right)_{\bar{s}} \times \bar{\eta}, \overline{\mathbb{Q}}_{\ell}\right)$. Similarly, since the complex $\mathcal{V}_{\xi, Z}^{\natural}$ is a direct sum of shifted lisse sheaves (see Definition 4.3.8), the canonical morphism $\mathcal{V}_{\xi, Z}^{\natural} \otimes^{L} R \Psi_{Z}\left(\overline{\mathbb{Q}}_{\ell}\right) \rightarrow R \Psi_{Z}\left(\mathcal{V}_{\xi, Z}^{\natural}\right)$ is an isomorphism in $D_{c}^{b}\left(Z_{\bar{s}} \times \bar{\eta}, \overline{\mathbb{Q}}_{\ell}\right)$. Hence, (4.4.2) can be rewritten as an isomorphism

$$
i_{\bar{s}}^{\min , *} R j_{\bar{s}, *}^{\min }\left(\left.\left.R \Psi_{\mathrm{X}_{\mathcal{H}}}\left(\mathcal{V}_{\xi}\right)\right|_{\left(\mathrm{X}_{\mathcal{H}}\right)_{\bar{s}}^{b}} \stackrel{\sim}{\rightarrow} R \Psi_{\mathrm{Z}}\left(\mathcal{V}_{\xi, Z}^{\natural}\right)\right|_{Z_{\bar{s}}^{b}}\right.
$$


in $D_{c}^{b}\left(Z_{\bar{s}}, \overline{\mathbb{Q}}_{\ell}\right)$, where $\left.R \Psi_{\mathrm{X}_{\mathcal{H}}}\left(\mathcal{V}_{\xi}\right)\right|_{\left(\mathrm{X}_{\mathcal{H}}\right)_{\bar{s}}^{b}}$ and $\left.R \Psi_{\mathrm{Z}}\left(\mathcal{V}_{\xi, Z}^{\natural}\right)\right|_{Z_{\bar{s}}^{b}}$ abusively denote their extensions by zero to the whole schemes $\left(\mathrm{X}_{\mathcal{H}}\right)_{\bar{s}}$ and $Z_{\bar{s}}$.

As $\mathcal{H} \subset \mathrm{G}(\hat{\mathbb{Z}})$ varies among principal levels, the attached $\mathcal{H}_{\mathrm{P}}:=\mathcal{H} \cap \mathrm{P}\left(\mathbb{A}^{\infty}\right)$, $\mathcal{H}_{\mathrm{U}}:=\mathcal{H} \cap \mathrm{U}\left(\mathbb{A}^{\infty}\right), \mathcal{H}_{\mathrm{M}}:=\mathcal{H}_{\mathrm{P}} / \mathcal{H}_{\mathrm{U}}$, and $\mathcal{H}_{h}:=\left(\mathrm{M}\left(\mathbb{A}^{\infty}\right) \rightarrow \mathrm{G}_{h}\left(\mathbb{A}^{\infty}\right)\right)\left(\mathcal{H}_{\mathrm{M}}\right)$ (see Definition 3.3.8 and Remark 4.3.3) vary accordingly among principal levels in the respective ambient groups. We can also consider a projective system of higher level analogues of $Z$, as $\mathcal{H}_{h}^{\prime}=\mathcal{H}_{h, \Phi}=\mathcal{H}_{h} \subset \mathrm{G}_{h}\left(\mathbb{A}^{\infty}\right)$ vary among principal levels, which we abusively denote as $\widehat{Z}$.

However, to formulate the boundary version of (4.4.1), it is better to consider the $G_{l}\left(\mathbb{A}^{\infty}\right)$-orbit of $\widehat{Z}$ (which is a disjoint union of locally closed subsets in the limit of the projective system $X^{\min }=\left\{X_{\mathcal{H}}^{\min }\right\}_{\mathcal{H}}$ parameterized by principal levels $\mathcal{H}$ ), which we abusively denote as $\mathrm{X}_{\mathrm{P}}$. The elements of $\mathrm{G}_{l}\left(\mathbb{A}^{\infty}\right)$ act by twisting the torus arguments in the cusp labels, without changing the associated filtration $Z$. The finite level object $X_{P, \mathcal{H}}$ is the disjoint union of all $Z$ (the strata of $X_{\mathcal{H}}^{\text {min }}$ ) parameterized by cusp labels $\left[\left(\mathrm{Z}_{\mathcal{H}}, \Phi_{\mathcal{H}}, \delta_{\mathcal{H}}\right)\right]$ with the same underlying $\mathcal{H}$-orbit $\mathrm{Z}_{\mathcal{H}}$, where $\mathrm{Z}_{\mathcal{H}} \otimes_{\mathbb{Z}} \mathbb{Q}$ has stabilizer $\mathrm{P} \otimes_{\mathbb{Q}} \mathbb{A}^{\infty}$. Such cusp labels $\left[\left(\mathrm{Z}_{\mathcal{H}}, \Phi_{\mathcal{H}}, \delta_{\mathcal{H}}\right)\right]$ are in bijection with $\mathrm{G}_{l}(\mathbb{Q}) \backslash \mathrm{G}_{l}\left(\mathbb{A}^{\infty}\right) / \mathcal{H}_{l}^{\prime}=\mathrm{G}_{l}(\mathbb{Q}) \backslash \mathrm{G}_{l}\left(\mathbb{A}^{\infty}\right) / \mathcal{H}_{l}$. The generic fiber $\left(\mathrm{X}_{\mathrm{P}}\right)_{\eta}$ admits an action of $\mathrm{P}\left(\mathbb{A}^{\infty}\right)$ with trivial restriction to $\mathrm{U}\left(\mathbb{A}^{\infty}\right)$, and the induced action of $\mathrm{M}\left(\mathbb{A}^{\infty}\right) \cong \mathrm{P}\left(\mathbb{A}^{\infty}\right) / \mathrm{U}\left(\mathbb{A}^{\infty}\right) \cong \mathrm{G}_{l}\left(\mathbb{A}^{\infty}\right) \times \mathrm{G}_{h}\left(\mathbb{A}^{\infty}\right)$ is the combination of the action of $G_{l}\left(\mathbb{A}^{\infty}\right)$ as above and the action of $G_{h}\left(\mathbb{A}^{\infty}\right)$ on $\widehat{Z}_{\eta}$.

Since $\mathbf{P}^{+} / \mathbb{R}_{>0}^{\times} \cong \mathrm{G}_{l}(\mathbb{R}) / \mathcal{U}_{l, \infty}$ (where $\mathcal{U}_{l, \infty}$ is the stabilizer of some element of $\mathbf{P}^{+}$) is a Riemannian symmetric space associated with $G_{l}(\mathbb{R})$, the group cohomology of $\Gamma$ can be computed using the cohomology of the manifold $\Gamma \backslash \mathbf{P}^{+} / \mathbb{R}_{>0}^{\times}$. By putting together the $\mathcal{V}_{\xi, Z}^{\natural}$ over each $Z$ at level $\mathcal{H}$, each of which being a cohomology complex of $\Gamma$, we obtain the cohomology complex over $\mathrm{G}_{l}(\mathbb{Q}) \backslash \mathrm{G}_{l}(\mathbb{A}) / \mathcal{U}_{l, \infty} \mathcal{H}_{l}$. Thus, by taking limit over all principal levels $\mathcal{H}$, we obtain a limit $\mathcal{V}_{\xi, \mathrm{P}}^{\natural}$ over $\mathrm{X}_{\mathrm{P}}$, which admits an admissible action of $\mathrm{G}_{l}\left(\mathbb{A}^{\infty}\right)$.

Suppose that $b \in \mathrm{B}\left(\mathrm{G} \otimes_{\mathbb{Z}} \mathbb{Q}_{p}\right)$ is the image of some $b_{\mathrm{P}} \in \mathrm{B}\left(\mathrm{P} \otimes_{\mathbb{Q}} \mathbb{Q}_{p}\right)$ with image $b_{\mathrm{M}}=\left(b_{l}, b_{h}\right)$ in $\mathrm{B}\left(\mathrm{M} \otimes_{\mathbb{Q}} \mathbb{Q}_{p}\right) \cong \mathrm{B}\left(\mathrm{G}_{l} \otimes_{\mathbb{Q}} \mathbb{Q}_{p}\right) \times \mathrm{B}\left(\mathrm{G}_{h} \otimes_{\mathbb{Q}} \mathbb{Q}_{p}\right)$. Then we can define $\left(\mathrm{X}_{\mathrm{P}}\right)_{s}^{b_{h}}$ to be the $\mathrm{G}_{l}\left(\mathbb{A}^{\infty}\right)$-orbit of $\widehat{Z}_{s}^{b_{h}}$, the preimage of $Z_{s}^{b_{h}}$, and write symbolically

$$
\left.i_{\mathrm{P}, \bar{s}}^{\min , *} R j_{\bar{s}, *}^{\min }\left(\left.R \Psi_{\mathrm{X}}\left(\mathcal{V}_{\xi}\right)\right|_{\mathrm{X}_{\bar{s}}^{b}}\right) \stackrel{\sim}{\rightarrow} R \Psi_{\mathrm{X}_{\mathrm{P}}}\left(\mathcal{V}_{\xi, \mathrm{P}}^{\natural}\right)\right|_{\left(\mathrm{X}_{\mathrm{P}}\right)_{\bar{s}}^{b_{h}}} .
$$

By applying the analogue of (4.4.1) to $X_{P}$, we obtain the following equality

$$
\begin{aligned}
& \sum_{i}(-1)^{i}\left[H_{\mathrm{et}, c}^{i}\left(\left(\mathrm{X}_{\mathrm{P}}\right)_{\bar{s}}, i_{\mathrm{P}, \bar{s}}^{\min , *} R j_{\bar{s}, *}^{\min }\left(\left.R \Psi_{\mathrm{X}}\left(\mathcal{V}_{\xi}\right)\right|_{\mathrm{X}_{\bar{s}}^{b}}\right)\right)\right] \\
& =\sum_{j}(-1)^{j} \mathcal{E}_{b_{h}}\left(\left[H_{\mathrm{et}, c}^{j}\left(\lg _{\mathrm{P}}^{b_{h}}, \mathcal{V}_{\xi, \mathrm{P}}^{\natural}\right)\right]\right)
\end{aligned}
$$


between virtual representations of $\mathrm{G}_{l}\left(\mathbb{A}^{\infty}\right) \times \mathrm{G}_{h}\left(\mathbb{A}^{\infty, p}\right) \times \mathrm{G}_{h}\left(\mathbb{Q}_{p}\right)^{+} \times W_{K}$, where $\mathrm{G}_{h}\left(\mathbb{Q}_{p}\right)^{+}$is the submonoid of $\mathrm{G}_{h}\left(\mathbb{Q}_{p}\right)$ analogous to the submonoid $\mathrm{G}\left(\mathbb{Q}_{p}\right)^{+}$of $\mathrm{G}\left(\mathbb{Q}_{p}\right)$, where $\lg _{\mathrm{P}}^{b_{h}}$ is the analogous limit of Igusa varieties over $\left(\mathrm{X}_{\mathrm{P}}\right)_{\bar{s}}^{b_{h}}$ (whose pullback to $\widehat{Z}_{s}$ is the usual one associated with $\mathrm{G}_{h}$, over some chosen central leaf at the bottom level), where each $H_{\text {et, } c}^{j}\left(\lg _{\mathrm{P}}^{b_{h}}, \mathcal{V}_{\xi, \mathrm{P}}^{\natural}\right)$ admits an admissible/continuous action of $\mathrm{G}_{l}\left(\mathbb{A}^{\infty}\right) \times \mathrm{G}_{h}\left(\mathbb{A}^{\infty, p}\right) \times J_{b_{h}}\left(\mathbb{Q}_{p}\right) \times W_{K}$, and where

$\mathcal{E}_{b_{h}}: \operatorname{Groth}\left(\mathrm{G}_{l}\left(\mathbb{A}^{\infty}\right) \times \mathrm{G}_{h}\left(\mathbb{A}^{\infty, p}\right) \times J_{b_{h}}\left(\mathbb{Q}_{p}\right)\right) \rightarrow \operatorname{Groth}\left(\mathrm{G}_{l}\left(\mathbb{A}^{\infty}\right) \times \mathrm{G}_{h}\left(\mathbb{A}^{\infty}\right) \times W_{K}\right)$.

is the combination of the identity functor on $\operatorname{Groth}\left(\mathrm{G}_{l}\left(\mathbb{A}^{\infty}\right)\right)$ and the corresponding Mantovan functor for $\mathrm{G}_{h}$ (cf. [45, (6.20)]).

ThEOREM 4.4.7 (Cf. [51, Section 8, Theorem 22] and [52, Theorem 3.1], and also [45, Theorems 6.26 and 6.32]). For each $b \in \mathrm{B}\left(\mathrm{G} \otimes_{\mathbb{Z}} \mathbb{Q}_{p}\right)$, we have an equality

$$
\begin{aligned}
& \sum_{i}(-1)^{i}\left[H_{\mathrm{et}}^{i}\left(\mathrm{X}_{\bar{s}},\left.\left(R \Psi_{\mathrm{X}}\left(\mathcal{V}_{\xi}\right)\right)\right|_{\mathrm{X}_{\bar{s}}^{b}}\right)\right] \\
& =\sum_{\mathrm{P}} \operatorname{Ind}_{\mathrm{P}\left(\mathbb{A}^{\infty}\right)}^{\mathrm{G}\left(\mathbb{A}^{\infty}\right)}\left(\sum_{j}(-1)^{j} \mathcal{E}_{b_{h}}\left(\left[H_{\mathrm{et}, c}^{j}\left(\lg _{\mathrm{P}}^{b_{h}}, \mathcal{V}_{\xi, \mathrm{P}}^{\natural}\right)\right]\right)\right)
\end{aligned}
$$

between virtual representations of $\mathrm{G}\left(\mathbb{A}^{\infty, p}\right) \times \mathrm{G}\left(\mathbb{Q}_{p}\right)^{+} \times W_{K}$, where the sum over $\mathrm{P}$ runs over a complete (finite) set of representatives of conjugacy classes of (rational) parabolic subgroups of $\mathrm{G} \otimes_{\mathbb{Z}} \mathbb{Q}$ that is either (proper) maximal or $\mathrm{G} \otimes_{\mathbb{Z}} \mathbb{Q}$ itself such that $b \in \mathrm{B}\left(\mathrm{G} \otimes_{\mathbb{Z}} \mathbb{Q}_{p}\right)$ is the image of some $b_{\mathrm{P}} \in \mathrm{B}\left(\mathrm{P} \otimes_{\mathbb{Q}} \mathbb{Q}_{p}\right)$ with image $b_{\mathrm{M}}=\left(b_{l}, b_{h}\right)$ in $\mathrm{B}\left(\mathrm{M} \otimes_{\mathbb{Q}} \mathbb{Q}_{p}\right) \cong \mathrm{B}\left(\mathrm{G}_{l} \otimes_{\mathbb{Q}} \mathbb{Q}_{p}\right) \times \mathrm{B}\left(\mathrm{G}_{h} \otimes_{\mathbb{Q}} \mathbb{Q}_{p}\right)$. (Note that $b_{h}$ depends on $\mathrm{P}$.)

Overall, we have an equality

$$
\begin{aligned}
& \sum_{i}(-1)^{i}\left[H_{\mathrm{et}}^{i}\left(\mathrm{X}_{\bar{s}}, R \Psi_{\mathrm{X}}\left(\mathcal{V}_{\xi}\right)\right)\right] \\
& =\sum_{\mathrm{P}} \operatorname{Ind}_{\mathrm{P}\left(\mathbb{A}^{\infty}\right)}^{\mathrm{G}\left(\mathbb{A}^{\infty}\right)}\left(\sum_{b_{h} \in \mathrm{B}\left(\mathrm{G}_{h} \otimes \mathbb{Q} \mathbb{Q}_{p}\right)} \sum_{j}(-1)^{j} \mathcal{E}_{b_{h}}\left(\left[H_{\mathrm{et}, c}^{j}\left(\lg _{\mathrm{P}}^{b_{h}}, \mathcal{V}_{\xi, \mathrm{P}}^{\natural}\right)\right]\right)\right)
\end{aligned}
$$

between virtual representations of $\mathrm{G}\left(\mathbb{A}^{\infty, p}\right) \times \mathrm{G}\left(\mathbb{Q}_{p}\right)^{+} \times W_{K}$, and an equality

$$
\begin{aligned}
& \sum_{i}(-1)^{i}\left[H_{\mathrm{et}}^{i}\left(\mathrm{X}_{\bar{\eta}}, \mathcal{V}_{\xi}\right)\right] \\
& =\sum_{\mathrm{P}} \operatorname{Ind}_{\mathrm{P}\left(\mathbb{A}^{\infty}\right)}^{\mathrm{G}\left(\mathbb{A}^{\infty}\right)}\left(\sum_{b_{h} \in \mathrm{B}\left(\mathrm{G}_{h} \otimes_{\mathbb{Q}} \mathbb{Q}_{p}\right)} \sum_{j}(-1)^{j} \mathcal{E}_{b_{h}}\left(\left[H_{\mathrm{et}, c}^{j}\left(\lg _{\mathrm{P}}^{b_{h}}, \mathcal{V}_{\xi, \mathrm{P}}^{\natural}\right)\right]\right)\right)
\end{aligned}
$$


between virtual representations of $\mathrm{G}\left(\mathbb{A}^{\infty}\right) \times W_{K}$, where the sums over $\mathrm{P}$ run over a complete (finite) set of representatives of conjugacy classes of (rational) parabolic subgroups of $\mathrm{G} \otimes_{\mathbb{Z}} \mathbb{Q}$ that is either (proper) maximal or $\mathrm{G} \otimes_{\mathbb{Z}} \mathbb{Q}$ itself.

Proof. Let $\mathrm{X}^{\mathrm{min}}$ denote the limit of $\mathrm{X}_{\mathcal{H}}^{\min }$ over principal levels $\mathcal{H} \subset \mathrm{G}(\hat{\mathbb{Z}})$. By [37, Propositions A.5.8 and A.5.9], we have a stratification $\bigsqcup_{P}\left(G\left(\mathbb{A}^{\infty}\right) \cdot X_{P}\right)$ of $\mathrm{X}^{\mathrm{min}}$, where the disjoint union is over the same $\mathrm{P}$ 's as in the second paragraph of the statement of the theorem, and where $P\left(\mathbb{A}^{\infty}\right)$ is the stabilizer of $X_{P}$ in $\mathrm{G}\left(\mathbb{A}^{\infty}\right)$. By (2) of Proposition 2.1.2, the same are true over the fibers over $\bar{s}$. By Proposition 3.3.9, $\left(\mathrm{X}_{\mathrm{P}}\right)_{\bar{s}}$ contains some nonempty $\mathrm{Z}_{\bar{s}}^{b}$ only when $b \in \mathrm{B}\left(\mathrm{G} \otimes_{\mathbb{Z}} \mathbb{Q}_{p}\right)$ is the image of some $b_{\mathrm{P}} \in \mathrm{B}\left(\mathrm{P} \otimes_{\mathbb{Q}} \mathbb{Q}_{p}\right)$. Thus, we obtain the equality (4.4.8) between virtual representations of $\mathrm{G}\left(\mathbb{A}^{\infty, p}\right) \times \mathrm{G}\left(\mathbb{Q}_{p}\right)^{+} \times W_{K}$ by summing the parabolic inductions of (4.4.5) over the same P's as in the first paragraph of the statement of the theorem.

As in $[45,(6.24)]$, we have an equality

$$
\begin{aligned}
\sum_{i} & (-1)^{i}\left[H_{\mathrm{et}}^{i}\left(\mathrm{X}_{\bar{s}}, R \Psi_{\mathrm{X}}\left(\mathcal{V}_{\xi}\right)\right)\right] \\
= & \sum_{b \in \mathrm{B}\left(\mathrm{G} \otimes_{\mathbb{Q}} \mathbb{Q}_{p}\right)} \sum_{i}(-1)^{i}\left[H_{\mathrm{et}}^{i}\left(\mathrm{X}_{\bar{s}}^{b},\left.\left(R \Psi_{\mathrm{X}}\left(\mathcal{V}_{\xi}\right)\right)\right|_{\mathrm{X}_{\bar{s}}^{b}}\right)\right]
\end{aligned}
$$

between virtual representations of $\mathrm{G}\left(\mathbb{A}^{\infty}\right) \times W_{K}$. By combining the contributions from all $b \in \mathrm{B}\left(\mathrm{G} \otimes_{\mathbb{Z}} \mathbb{Q}_{p}\right)$, we obtain the equality (4.4.9) between virtual representations of $\mathrm{G}\left(\mathbb{A}^{\infty, p}\right) \times \mathrm{G}\left(\mathbb{Q}_{p}\right)^{+} \times W_{K}$. Thus, by [45, Corollary 5.20 and Remark 5.35], we obtain (4.4.10) as an equality between virtual representations of $\mathrm{G}\left(\mathbb{A}^{\infty, p}\right) \times \mathrm{G}\left(\mathbb{Q}_{p}\right)^{+} \times W_{K}$, which then extends to the desired equality between virtual representations of $\mathrm{G}\left(\mathbb{A}^{\infty}\right) \times W_{K}$ (cf. [51, Section 8]).

REMARK 4.4.12. The term $H_{\mathrm{et}}^{i}\left(\mathrm{X}_{\bar{s}},\left.\left(R \Psi_{\mathrm{X}}\left(\mathcal{V}_{\xi}\right)\right)\right|_{X_{\bar{s}}^{b}}\right)$ in (4.4.9) differs from both $H_{\mathrm{ett}}^{i}\left(\mathrm{X}_{\bar{s}}^{b},\left.\left(R \Psi_{\mathrm{X}}\left(\mathcal{V}_{\xi}\right)\right)\right|_{\mathrm{X}_{\bar{s}}^{b}}\right)$ and $H_{\mathrm{et}, c}^{i}\left(\mathrm{X}_{\bar{s}}^{b},\left.\left(R \Psi_{\mathrm{X}}\left(\mathcal{V}_{\xi}\right)\right)\right|_{\mathrm{X}_{\bar{s}}^{b}}\right)$ in general.

REMARK 4.4.13. Compared with its analogue for $\sum_{i}(-1)^{i}\left[H_{\mathrm{et}, c}^{i}\left(\mathrm{X}_{\bar{\eta}}, \mathcal{V}_{\xi}\right)\right]$ in $[45,(6.33)]$, the formula (4.4.10) is more elaborate and contains the additional (boundary cohomology) terms parameterized by all proper maximal parabolic subgroups $\mathrm{P}$ of $\mathrm{G} \otimes_{\mathbb{Z}} \mathbb{Q}$, although (in theory) these two formulas imply each other by duality.

REMARK 4.4.14. Here is an alternative argument for establishing the equality (4.4.10) (which does not rely on Proposition 3.3.9 and Theorem 4.3.16): By applying the analogues of [45, Corollary 5.20, Remark 5.35, and (6.24)] 
and (4.4.1) for $X_{P}$, we obtain an equality

$$
\begin{aligned}
& \sum_{i}(-1)^{i}\left[H_{\text {et }, c}^{i}\left(\left(\mathrm{X}_{\mathrm{P}}\right)_{\bar{\eta}}, \mathcal{V}_{\xi, \mathrm{P}}^{\natural}\right)\right] \\
& =\sum_{b_{h} \in \mathrm{B}\left(\mathrm{G}_{h} \otimes_{\mathbb{Q}} \mathbb{Q}_{p}\right)} \sum_{j}(-1)^{j} \mathcal{E}_{b_{h}}\left(\left[H_{\text {ét }, c}^{j}\left(\lg _{\mathrm{P}}^{b_{h}}, \mathcal{V}_{\xi, \mathrm{P}}^{\natural}\right)\right]\right)
\end{aligned}
$$

between virtual representations of $\mathrm{G}_{l}\left(\mathbb{A}^{\infty}\right) \times \mathrm{G}_{h}\left(\mathbb{A}^{\infty, p}\right) \times \mathrm{G}_{h}\left(\mathbb{Q}_{p}\right)^{+} \times W_{K}$, which extends to an equality between virtual representations of $\mathrm{G}_{l}\left(\mathbb{A}^{\infty}\right) \times \mathrm{G}_{h}\left(\mathbb{A}^{\infty}\right) \times W_{K}$, by the same explanation as in [51, Section 8]. By Theorem 4.3.10 (the original theorem of Pink's), we can rewrite (4.4.15) as an equality

$$
\begin{aligned}
& \sum_{i}(-1)^{i}\left[H_{\text {ét, } c}^{i}\left(\left(\mathrm{X}_{\mathrm{P}}\right)_{\bar{\eta}}, i_{\mathrm{P}, \bar{\eta}}^{\min , *} R j_{\bar{\eta}, *}^{\min } \mathcal{V}_{\xi}\right)\right] \\
& =\sum_{b_{h} \in \mathrm{B}\left(\mathrm{G}_{h} \otimes_{\mathbb{Q}} \mathbb{Q}_{p}\right)} \sum_{j}(-1)^{j} \mathcal{E}_{b_{h}}\left(\left[H_{\text {ét, }, c}^{j}\left(\lg _{\mathrm{P}}^{b_{h}}, \mathcal{V}_{\xi, \mathrm{P}}^{\natural}\right)\right]\right)
\end{aligned}
$$

between virtual representations of $\mathrm{G}_{l}\left(\mathbb{A}^{\infty}\right) \times \mathrm{G}_{h}\left(\mathbb{A}^{\infty}\right) \times W_{K}$. Thus, we obtain the desired equality (4.4.10) by summing the parabolic inductions of (4.4.16) over the same P's as in the second paragraph of Theorem 4.4.7. However, such an argument cannot isolate the contribution of a single Newton stratum as in (4.4.8).

4.5. Morel's formula. The goal of this subsection is to generalize Morel's results concerning restrictions to boundary strata of intersection complexes of automorphic étale sheaves in [58, Section 5.2], [59, Section 4.2], and [61, Section 1.4] (see also [60]), and their variants in [80, Section 9], [81], and [82], to the case of general tensor products of automorphic étale sheaves with well-positioned pure perverse sheaves. (See Theorems 4.5.26 and 4.5.37 below.)

We shall resume the context of Section 4.3, with Assumption 4.3.1, because our generalization of Morel's results depends on our generalization of Pink's formula there, just as the original results of Morel's depend on the original formula of Pink's. For simplicity, we shall make the following assumption in this subsection:

Assumption 4.5.1. We are in Cases $(\mathrm{Sm}),(\mathrm{Nm})$, or $(\mathrm{Spl})$, and $C \rightarrow \mathrm{Z}$ is an abelian scheme torsor for each Z.

Recall that, in Section 4.3, we have extended the construction in [45, Proposition 3.2] to a functor from $D^{b}\left(\mathrm{G} \otimes_{\mathbb{Z}} \mathbb{Q}, \overline{\mathbb{Q}}_{\ell}\right)$ to $D_{c}^{b}\left(\mathbf{X}_{\mathcal{H}}, \overline{\mathbb{Q}}_{\ell}\right)$, and we have similar constructions over each stratum $\mathbf{Z}$ of $\mathbf{X}_{\mathcal{H}}^{\min }$.

Let $\overline{\mathbb{Q}}$ denote the algebraic closure of $\mathbb{Q}$ in $\mathbb{C}$, and fix a choice of a field homomorphism $\overline{\mathbb{Q}} \hookrightarrow \overline{\mathbb{Q}}_{\ell}$. Since we will be working with weights, it is more 
convenient to work with $D^{b}\left(\mathrm{G} \otimes_{\mathbb{Z}} \overline{\mathbb{Q}}, \overline{\mathbb{Q}}_{\ell}\right)$, which is isomorphic to $D^{b}\left(\mathrm{G} \otimes_{\mathbb{Z}} \mathbb{Q}\right.$, $\overline{\mathbb{Q}}_{\ell}$ ) (via the above choice of $\overline{\mathbb{Q}} \hookrightarrow \overline{\mathbb{Q}}_{\ell}$ ).

The $h_{0}: \mathbb{C} \rightarrow \operatorname{End}_{\mathcal{O} \otimes_{\mathbb{Z}} \mathbb{R}}\left(L \otimes_{\mathbb{Z}} \mathbb{R}\right)$ in Assumption 2.1.1 (cf. [36, Definition 1.2.1.3]) induces by restriction a central cocharacter $\mathbf{G}_{\mathrm{m}} \otimes_{\mathbb{Z}} \mathbb{R} \rightarrow \mathrm{G} \otimes_{\mathbb{Z}} \mathbb{R}$, whose reciprocal descends to a cocharacter $w: \mathbf{G}_{\mathrm{m}} \otimes_{\mathbb{Z}} \mathbb{Q} \rightarrow \mathrm{G} \otimes_{\mathbb{Z}} \mathbb{Q}$ (cf. [14, 1.1.1] for the general definition). For simplicity, we shall also denote by $w$ the base change $\mathbf{G}_{\mathrm{m}} \otimes_{\mathbb{Z}} \overline{\mathbb{Q}} \rightarrow \mathrm{G} \otimes_{\mathbb{Z}} \overline{\mathbb{Q}}$.

Definition 4.5.2. We say a complex $V \in D^{b}\left(\mathrm{G} \otimes_{\mathbb{Z}} \overline{\mathbb{Q}}, \overline{\mathbb{Q}}_{\ell}\right)$ is pure of weight $-a \in \mathbb{Z}$ if, by pullback under the cocharacter $w$, the group $\mathbf{G}_{\mathrm{m}} \otimes_{\mathbb{Z}} \overline{\mathbb{Q}}$ acts on $\mathcal{H}^{n}(V)$ via the character $x \mapsto x^{a+n}$, for each $n \in \mathbb{Z}$.

EXAMPLE 4.5.3. The representation $L \otimes_{\mathbb{Z}} \overline{\mathbb{Q}}_{\ell}$ of $G \otimes_{\mathbb{Z}} \overline{\mathbb{Q}}$ (with its tautological action, placed in degree zero) is pure of weight 1.

Lemma 4.5.4. Suppose $V \in D^{b}\left(\mathrm{G} \otimes_{\mathbb{Z}} \overline{\mathbb{Q}}, \overline{\mathbb{Q}}_{\ell}\right)$ is pure of weight $-a \in \mathbb{Z}$ as in Definition 4.5.2, with associated complex $\mathcal{V} \in D_{c}^{b}\left(\mathbf{X}_{\mathcal{H}}, \overline{\mathbb{Q}}_{\ell}\right)$. For each functorial point $\bar{s}=\operatorname{Spec}(k) \rightarrow \mathrm{S}$, where $k$ is a perfect field of characteristic $p$, the pullback $\mathcal{V}_{\bar{s}}$ of $\mathcal{V}$ to $\left(\mathrm{X}_{\mathcal{H}}\right)_{\bar{s}}$ is mixed of weight a (cf. [15, Definition 6.2.2]). More precisely, $\mathcal{H}^{n}\left(\mathcal{V}_{\bar{s}}\right)$ is lisse and pointwise pure of weight $a+n$, for each $n \in \mathbb{Z}$ (see $[15$, Definition 1.2.2]).

Proof. This follows from [45, Proposition 3.2] and [72, Proposition 5.6.2 and Lemma 5.6.6].

REMARK 4.5.5. Beware that in general $\mathcal{V}$ is not pure (as in [15, Definition 6.2.4]) when $\left(\mathrm{X}_{\mathcal{H}}\right)_{\bar{s}}$ is not smooth (over $\left.\bar{s}\right)$. Nevertheless, the restriction of $\mathcal{V}_{\bar{s}}$ to every smooth subscheme of $\left(\mathrm{X}_{\mathcal{H}}\right)_{\bar{s}}$ is pure of weight $a$ (see [15, Example 6.2.5(b)]).

LEMMA 4.5.6. Consider any $t=\operatorname{Spec}(k) \rightarrow \mathrm{S}$, where $k$ is a field of characteristic $p$ that is either algebraically closed or finite. Let $V \in D^{b}\left(\mathrm{G} \otimes_{\mathbb{Z}} \overline{\mathbb{Q}}\right.$, $\left.\overline{\mathbb{Q}}_{\ell}\right)$ be pure of weight $-a \in \mathbb{Z}$, with associated $\mathcal{V} \in D_{c}^{b}\left(\mathbf{X}_{\mathcal{H}}, \overline{\mathbb{Q}}_{\ell}\right)$, whose pullback to $\left(\mathrm{X}_{\mathcal{H}}\right)_{t}$ we abusively denote by the same symbols. Let $\mathcal{F}$ be a pure shifted perverse sheaf of weight $b \in \mathbb{Z}$ over $\left(\mathrm{X}_{\mathcal{H}}\right)_{t}$. Then $\mathcal{V} \otimes^{L} \mathcal{F}$ is pure of weight $a+b$, and is isomorphic to a direct sum of shifted perverse sheaves. If $V$ is concentrated in degree zero, and if $\mathcal{F}$ is a perverse sheaf, then $\mathcal{V} \otimes^{L} \mathcal{F}$ is also a perverse sheaf.

Proof. The tensor product $\mathcal{V} \otimes^{L} \mathcal{F}$ is isomorphic to a direct sum of shifted perverse sheaves because the fibered category of perverse sheaves is a category module over the symmetric monoidal fibered category of lisse sheaves. Consider $V^{\vee}:=\operatorname{RHom}\left(V, \overline{\mathbb{Q}}_{\ell}\right)$ in $D^{b}\left(\mathrm{G} \otimes_{\mathbb{Z}} \overline{\mathbb{Q}}, \overline{\mathbb{Q}}_{\ell}\right)$, with associated 
$\mathcal{V}^{\vee}:=R \mathcal{H} \operatorname{om}\left(\mathcal{V}, \overline{\mathbb{Q}}_{\ell}\right)$ in $D_{c}^{b}\left(\mathbf{X}_{\mathcal{H}}, \overline{\mathbb{Q}}_{\ell}\right)$. In order to show that $\mathcal{V} \otimes^{L} \mathcal{F}$ is pure of weight $a+b$, it suffices to note that $\mathcal{V} \otimes^{L} \mathcal{F}$ is mixed of weight $\leqslant a+b$ (by Lemma 4.5.4), and that

$$
\begin{aligned}
D_{\left(\mathrm{X}_{\mathcal{H}}\right)_{t}}\left(\mathcal{V} \otimes^{L} \mathcal{F}\right) & \cong R \mathcal{H o m}\left(\mathcal{V} \otimes^{L} \mathcal{F}, \mathcal{K}_{\left(\mathrm{X}_{\mathcal{H})_{t}}\right)}\right. \\
& \cong \mathcal{V}^{\vee} \otimes^{L} R \mathcal{H} \operatorname{Hom}\left(\mathcal{F}, \mathcal{K}_{\left(\mathrm{X}_{\mathcal{H}}\right)_{t}}\right) \cong \mathcal{V}^{\vee} \otimes^{L} D_{\left(\mathrm{X}_{\mathcal{H}}\right)_{t}}(\mathcal{F})
\end{aligned}
$$

is mixed of weight $\leqslant-a-b$. (See [6, 5.1.13 and 5.1.14].) If $V$ is concentrated in degree zero, then $\mathcal{V}$ is a lisse sheaf concentrated in degree zero, and hence $\mathcal{V} \otimes^{L} \mathcal{F}$ is a perverse sheaf if $\mathcal{F}$ is.

Let $Z$ be a stratum of $X_{\mathcal{H}}^{\min }$, which is associated with some (rational) parabolic subgroup $\mathrm{P}$ of $\mathrm{G} \otimes_{\mathbb{Z}} \mathbb{Q}$, as in Lemma 3.3.6, which is the stabilizer of a symplectic filtration $\mathrm{V}=\left\{\mathrm{V}_{-i}\right\}_{i \in \mathbb{Z}}$ of $L \otimes_{\mathbb{Z}} \mathbb{Q}$ such that $\mathrm{V}_{-2} \otimes_{\mathbb{Q}} \mathbb{A}^{\infty}$ lies in the $\mathcal{H}$-orbit of $Z_{-2} \otimes_{\mathbb{Z}} \mathbb{Q}$ for some representative $\mathrm{Z}=\left\{Z_{-i}\right\}_{i \in \mathbb{Z}}$ of $Z_{\mathcal{H}}$.

Definition 4.5.7. Consider a collection $\mathscr{C}=\left\{Z^{(c)}\right\}_{c}$ of strata of $X_{\mathcal{H}}^{\min }$ containing the open stratum $X_{\mathcal{H}}$. Then the incidence relation among the strata of $X_{\mathcal{H}}^{\min }$ induces a partial order on the indices $c$ by declaring that $c \leqslant c^{\prime}$ whenever $\bar{Z}^{(c)} \subset \bar{Z}^{\left(c^{\prime}\right)}$. We say that $\mathscr{C}$ is a chain if such an induced partial ordering is a total ordering. We say that a chain $\mathscr{C}$ ends with Z, in which case we write $\mathscr{C} \triangleright Z$, if $Z$ is the (unique) minimal element in $\mathscr{C}$.

LEMMA 4.5.8. Suppose $\mathrm{V}_{-2}$ and $\mathrm{Z}_{-2}$ are as above, and suppose $\mathscr{C}=\left\{\mathrm{Z}^{(c)}\right\}_{c} \triangleright \mathbf{Z}$ as in Definition 4.5.7. Let $c_{0}>c_{1}>\cdots>c_{r}$ be a total ordering of all the indices of $\mathscr{C}$, so that $\mathrm{X}_{\mathcal{H}}=\mathrm{Z}^{\left(c_{0}\right)}$ and $\mathrm{Z}=\mathrm{Z}^{\left(c_{r}\right)}$. Then there exists a filtration

$$
0=\mathrm{V}_{-2}^{\left(c_{0}\right)} \subsetneq \mathrm{V}_{-2}^{\left(c_{1}\right)} \subsetneq \cdots \subsetneq \mathrm{V}_{-2}^{\left(c_{r}\right)}=\mathrm{V}_{-2},
$$

which induces by base change a filtration

$0=V_{-2}^{\left(c_{0}\right)} \otimes_{\mathbb{Q}} \mathbb{A}^{\infty} \subsetneq \mathrm{V}_{-2}^{\left(c_{1}\right)} \otimes_{\mathbb{Q}} \mathbb{A}^{\infty} \subsetneq \cdots \subsetneq \mathrm{V}_{-2}^{\left(c_{r}\right)} \otimes_{\mathbb{Q}} \mathbb{A}^{\infty}=\mathrm{V}_{-2} \otimes_{\mathbb{Q}} \mathbb{A}^{\infty}=\mathrm{Z}_{-2} \otimes_{\mathbb{Z}} \mathbb{Q}$,

whose $\mathcal{H}$-orbit depends only on $\mathscr{C}$. The stabilizer $\mathrm{Q}$ of (4.5.9) in $\mathrm{G} \otimes_{\mathbb{Z}} \mathbb{Q}$ is a parabolic subgroup satisfying the following conditions (cf. Definition 3.3.8):

(1) $\mathrm{U} \subset \mathrm{Q} \subset \mathrm{P}$, and so the unipotent radical $\mathrm{U}_{\mathrm{Q}}$ of $\mathrm{Q}$ contains $\mathrm{U}$, and the Levi quotient $\mathrm{M}_{\mathrm{Q}}:=\mathrm{Q} / \mathrm{U}_{\mathrm{Q}}$ can be identified with the Levi quotient of the parabolic subgroup $\mathrm{Q} / \mathrm{U}$ of $\mathrm{M}$.

(2) There is a canonical homomorphism $\mathrm{Q} / \mathrm{U} \rightarrow \mathrm{G}_{h}$, which is surjective and canonically splits. 
(3) $\mathrm{Q}_{l}:=\operatorname{ker}\left((\mathrm{Q} / \mathrm{U}) \rightarrow \mathrm{G}_{h}\right)$ is a (rational) parabolic subgroup of $\mathrm{G}_{l}$, with unipotent radical $\mathrm{U}_{\mathrm{Q}_{l}} \cong \mathrm{U}_{\mathrm{Q}} / \mathrm{U}$ and Levi quotient $\mathrm{M}_{\mathrm{Q}_{l}}:=\mathrm{Q}_{l} / \mathrm{U}_{\mathrm{Q}_{l}}$.

Conversely, any (rational) parabolic subgroup of $G \otimes_{\mathbb{Z}} \mathbb{Q}$ satisfying these conditions define a chain linking $\mathbf{X}_{\mathcal{H}}$ and $\mathrm{Z}$ as in Definition 4.5.7.

Proof. Since the incidence relations among the strata of $Z^{\mathrm{min}}$ are the same as their restrictions to the characteristic zero fibers, the existence of the filtrations (4.5.9) and (4.5.10) follows from [36, Theorem 7.2.4.1(4) and Lemma 5.4.2.11]. The bijection between the filtrations as in (4.5.9) and the parabolic subgroups Q of $\mathrm{G} \otimes_{\mathbb{Z}} \mathbb{Q}$ satisfying the above conditions then follows from [37, Proposition A.5.8 and Lemma A.4.3].

REMARK 4.5.11. By Lemma 4.5.8, such chains correspond to the strata of the reductive Borel-Serre compactification of (the analytification of) $\mathrm{X}_{\mathcal{H}} \otimes_{R_{0}} \mathbb{C}$ above the stratum $Z \otimes_{R_{0}} \mathbb{C}$ of $X_{\mathcal{H}}^{\min } \otimes_{R_{0}} \mathbb{C}$, where the base changes are defined by any ring homomorphism $R_{0} \rightarrow \mathbb{C}$. (See [9, Sections III.6 and III.10, and Propositions III.15.2 and III.15.4].)

DEFINITION 4.5.12. For each $\mathscr{C}$ and $\mathrm{Q}$ as in Lemma 4.5.8, we define

$$
r(\mathrm{Q}):=r(\mathscr{C}):=(\# \mathscr{C})-1 .
$$

We also define $\Gamma_{\mathrm{Q}}$ to be the image of $\Gamma \cap \mathrm{Q}_{l}(\mathbb{Q})$ (see Definition 4.3.4) under the canonical morphism $\mathrm{Q}_{l}(\mathbb{Q}) \rightarrow \mathrm{M}_{\mathrm{Q}_{l}}(\mathbb{Q})$, and $\mathfrak{u}_{\mathrm{Q}}:=\operatorname{Lie} \mathrm{U}_{\mathbf{Q}}\left(\mathbb{Q}_{\ell}\right)$.

LEMMA 4.5.14. For any other choice $\mathrm{Q}^{\prime}$ instead of $\mathrm{Q}$ as in Lemma 4.5.8, with $\Gamma_{\mathrm{Q}^{\prime}}$ and $\mathfrak{u}_{\mathrm{Q}^{\prime}}$ defined as in Definition 4.5.12, there are isomorphisms $\Gamma_{\mathrm{Q}} \stackrel{\sim}{\rightarrow} \Gamma_{\mathrm{Q}^{\prime}}$ and $\mathfrak{u}_{\mathrm{Q}} \stackrel{\sim}{\rightarrow} \mathfrak{u}_{\mathrm{Q}^{\prime}}$ of groups and $\ell$-adic analytic Lie groups, respectively, inducing isomorphisms between their cohomology.

Proof. This is because (4.5.9) determines and is determined by (4.5.10), and the $\mathcal{H}$-orbit of the latter depends only on $\mathscr{C}$.

Let $\mathscr{C}$ and $\mathrm{Q}$ be as in Lemma 4.5.8. For each $0 \leqslant i \leqslant r(\mathrm{Q})$, the stabilizer $\mathrm{P}^{(i)}$ of $\mathrm{V}_{-2}^{(i)}$ in $\mathrm{G} \otimes_{\mathbb{Z}} \mathbb{Q}$ is a parabolic subgroup associated with $Z^{\left(c_{i}\right)}$ as in Lemma 3.3.6. By definition, we have $\mathrm{Q}=\bigcap_{0 \leqslant i \leqslant r(\mathrm{Q})} \mathrm{P}^{(i)}$. Moreover, there exists a homomorphism

$$
s^{\mathrm{Q}}: \prod_{0 \leqslant i \leqslant r(\mathrm{Q})}\left(\mathbf{G}_{\mathrm{m}} \otimes_{\mathbb{Z}} \overline{\mathbb{Q}}\right) \rightarrow \mathrm{Q} \otimes_{\mathbb{Q}} \overline{\mathbb{Q}}
$$

which induces a cocharacter

$$
s^{\mathrm{Q},(i)}: \mathbf{G}_{\mathrm{m}} \otimes_{\mathbb{Z}} \overline{\mathbb{Q}} \rightarrow \mathrm{Q} \otimes_{\mathbb{Q}} \overline{\mathbb{Q}}
$$


from the $i$ th factor such that $s^{\mathrm{Q},(i)}$ acts:

(1) on $\mathrm{V}_{-2}^{\left(c_{i}\right)} \otimes_{\mathbb{Q}} \overline{\mathbb{Q}}$ by $x \mapsto x^{2}$;

(2) on $\left(\left(\mathrm{V}_{-2}^{\left(c_{i}\right)}\right)^{\perp} / \mathrm{V}_{-2}^{\left(c_{i}\right)}\right) \otimes_{\mathbb{Q}} \overline{\mathbb{Q}}$ by $x \mapsto x$; and

(3) on $\left(\left(L \otimes_{\mathbb{Z}} \mathbb{Q}\right) /\left(\mathrm{V}_{-2}^{\left(c_{i}\right)}\right)^{\perp}\right) \otimes_{\mathbb{Q}} \overline{\mathbb{Q}}$ by $x \mapsto 1$;

for each $0 \leqslant i \leqslant r(\mathrm{Q})$. (These are base changes of the graded pieces for the filtration $0=\mathrm{V}_{-3}^{\left(c_{i}\right)} \subset \mathrm{V}_{-2}^{\left(c_{i}\right)} \subset\left(\mathrm{V}_{-2}^{\left(c_{i}\right)}\right)^{\perp}=\mathrm{V}_{-1}^{\left(c_{i}\right)} \subset \mathrm{V}_{0}^{\left(c_{i}\right)}=L \otimes_{\mathbb{Z}} \mathbb{Q}$.) Such homomorphisms (4.5.15) and (4.5.16) exist because the filtration (4.5.9) splits. Moreover, the Levi quotient $\mathrm{Q} \rightarrow \mathrm{M}_{\mathrm{Q}}$ admits a splitting with image in the centralizer of the image of the homomorphism (4.5.15), or equivalently the centralizer of the images of the cocharacters (4.5.16), for all $0 \leqslant i \leqslant r(\mathrm{Q})$. From now on, we shall also view $\mathrm{M}_{\mathrm{Q}}$ as a Levi subgroup of $\mathrm{Q}$ using such a splitting.

For each $a \in \mathbb{Z}$, for each $0 \leqslant i \leqslant r(\mathrm{Q})$, and for each $V \in D^{b}\left(\mathrm{M}_{\mathrm{Q}} \otimes_{\mathbb{Z}} \overline{\mathbb{Q}}, \overline{\mathbb{Q}}_{\ell}\right)$, which we canonically view as an object in $D^{b}\left(\mathbf{Q} \otimes_{\mathbb{Q}} \overline{\mathbb{Q}}, \overline{\mathbb{Q}}_{\ell}\right)$ by pullback under the canonical homomorphism $\mathrm{Q} \rightarrow \mathrm{M}_{\mathrm{Q}}$, we define

$$
w_{\geqslant a}^{\mathrm{Q},(i)}(V):=\sum_{a^{\prime} \geqslant a}\left(\text { subcomplex of } V \text { on which } s^{\mathrm{Q},(i)} \text { acts by } x \mapsto x^{a^{\prime}}\right)
$$

and

$$
w_{<a}^{\mathrm{Q},(i)}(V):=\sum_{a^{\prime}<a}\left(\text { subcomplex of } V \text { on which } s^{\mathrm{Q},(i)} \text { acts by } x \mapsto x^{a^{\prime}}\right),
$$

which are again objects of $D^{b}\left(\mathrm{M}_{\mathrm{Q}} \otimes_{\mathbb{Z}} \overline{\mathbb{Q}}, \overline{\mathbb{Q}}_{\ell}\right)$, because $\mathrm{M}_{\mathrm{Q}}$ (as a Levi subgroup of Q) lies in the centralizer of the image of $s^{\mathrm{Q},(i)}$. Since (4.5.15) is the product of (4.5.16) as $i$ varies, the truncation operators as in (4.5.17) and (4.5.18) also commute with each other as $i$ varies.

For each $0 \leqslant i \leqslant r(\mathrm{Q})$, consider

$$
d_{\mathrm{Q},(i)}:=\operatorname{dim}\left(Z_{\eta}^{\left(c_{i}\right)}\right)
$$

(which does not depend on the choice of S). For each $a \in \mathbb{Z}$ and each $V \in$ $D^{b}\left(\mathrm{M}_{\mathrm{Q}} \otimes_{\mathbb{Q}} \overline{\mathbb{Q}}, \overline{\mathbb{Q}}_{\ell}\right)$, consider the subcomplex

$$
V_{\mathrm{Q}}^{\leqslant a}:=w_{\geqslant d_{\mathrm{Q},(r(\mathrm{Q}))}-a}^{\mathrm{Q},(r(\mathrm{Q}))} w_{<d_{\mathrm{Q},(r(\mathrm{Q})-1)}-a}^{\mathrm{Q},(r(\mathrm{Q})-1)} \cdots w_{<d_{\mathrm{Q},(2)}-a}^{\mathrm{Q},(2)} w_{<d_{\mathrm{Q},(1)}-a}^{\mathrm{Q},(1)}(V)
$$

in $D^{b}\left(\mathrm{M}_{\mathrm{Q}} \otimes_{\mathbb{Z}} \overline{\mathbb{Q}}, \overline{\mathbb{Q}}_{\ell}\right)$. The assignment of $V_{\mathrm{Q}}^{\leqslant a}$ to $V$ defines an exact functor

$$
(\cdot)_{\mathrm{Q}}^{\leqslant a}: D^{b}\left(\mathrm{M}_{\mathrm{Q}} \otimes_{\mathbb{Z}} \overline{\mathbb{Q}}, \overline{\mathbb{Q}}_{\ell}\right) \rightarrow D^{b}\left(\mathrm{M}_{\mathrm{Q}} \otimes_{\mathbb{Z}} \overline{\mathbb{Q}}, \overline{\mathbb{Q}}_{\ell}\right) .
$$


Definition 4.5.22. Let $\mathscr{C}$ and $\mathrm{Q}$ be as in Lemma 4.5.8, and let $\Gamma_{\mathrm{Q}}$ and $\mathfrak{u}_{\mathrm{Q}}$ be as in Definition 4.5.12. For each $a \in \mathbb{Z}$, and for each object $V$ in $D^{b}\left(\mathrm{G} \otimes_{\mathbb{Z}} \mathbb{Q}, \overline{\mathbb{Q}}_{\ell}\right)$, we define

$$
V_{\mathscr{C}}^{\natural, \leqslant a}:=R \operatorname{Inv}\left(\Gamma_{\mathrm{Q}},\left(R \operatorname{Inv}\left(\mathfrak{u}_{\mathrm{Q}},\left.V\right|_{\mathrm{Q}}\right)\right)_{\mathrm{Q}}^{\leqslant a}\right),
$$

which is a complex in $D^{b}\left(\mathrm{G}_{h} \otimes_{\mathbb{Z}} \overline{\mathbb{Q}}, \overline{\mathbb{Q}}_{\ell}\right)$, whose isomorphism class depends only on $\mathscr{C}$, but not on the choice of $\mathrm{Q}$, by Lemma 4.5.14. Then the constructions in [45, Section 3.1] and [72, Sections 1 and 4.9] associate a complex $\mathcal{V}_{\mathscr{C}}^{\natural, \leqslant a}$ in $D_{c}^{b}\left(Z, \overline{\mathbb{Q}}_{\ell}\right)$ (cf. Remark 4.3.7 and Definition 4.3.8) with $V_{\mathscr{C}}^{\natural, \leqslant a}$.

We need one last technical preparation:

LEMMA 4.5.24. Suppose $\mathrm{T}=\operatorname{Spec}(k)$, where $k$ is a field that is either algebraically closed or finite. Let $\mathrm{Y}$ be a well-positioned subset of $\left(\mathrm{X}_{\mathcal{H}}\right)_{\mathrm{T}}$, with associated $Y^{\natural}=\left\{Y_{Z}^{\natural}\right\}_{Z}$ as in Definition 2.2.1, and with partial minimal and toroidal compactifications $\mathrm{Y}^{\mathrm{min}}$ and $\mathrm{Y}_{\Sigma}^{\text {tor }}$ as in Definition 2.3.1 and Theorem 2.3.2. Suppose that $\mathcal{F}$ is a perverse sheaf over $\mathrm{Y}_{\Sigma}^{\text {tor }}$, equipped with a collection $\mathcal{F}^{\natural}=\left\{\left(\mathcal{F}_{Z}^{\natural}, \iota_{Z}\right)\right\}_{Z}$ as in Definition 4.1.3, such that $\mathcal{F}_{Z}^{\natural}\left[d^{Z}\right]$ is a perverse sheaf over $\mathrm{Z}$, where $d^{\mathrm{Z}}:=\operatorname{dim}\left(\left(\mathrm{X}_{\mathcal{H}}\right)_{\mathrm{T}}\right)-\operatorname{dim}\left(\mathrm{Z}_{\mathrm{T}}\right)$, for each $\mathrm{Z}$.

Now let us fix the choice of $\mathrm{Z}$. Suppose we have the morphisms

$$
\mathrm{Z}^{\mathrm{tor}} \rightarrow \mathrm{Z}^{\min } \stackrel{\sim}{\rightarrow} \overline{\mathrm{Z}} \rightarrow \mathrm{X}_{\mathcal{H}}^{\min }
$$

which induce

$$
\mathrm{Y}_{\mathrm{Z}}^{\text {tor }} \rightarrow \mathrm{Y}_{\mathrm{Z}}^{\min } \stackrel{\sim}{\rightarrow} \overline{\mathrm{Y}}_{\mathrm{Z}} \rightarrow \mathrm{Y}^{\min }
$$

as in Lemma 2.3.16 and its proof. Then, under the assumption (see Assumptions 4.3.1 and 4.5.1) that $Y_{C^{\prime}}^{\natural} \rightarrow Y_{Z^{\prime}}^{\natural}$ is an abelian scheme torsor (of constant relative dimension) for each stratum $Z^{\prime}$ of $Z^{\min }$, there exists a wellpositioned perverse sheaf $\mathcal{G}$ over $Z^{\text {tor }}$, equipped with a collection $\mathcal{G}^{\natural}=\left\{\left(\mathcal{G}_{Z^{\prime}}^{\natural}\right.\right.$, $\left.\left.\iota_{Z^{\prime}}^{\prime}\right)\right\}_{Z^{\prime}}$ indexed by the strata $Z^{\prime}$ in $\bar{Z}$, such that $\mathcal{G}_{Z^{\prime}}^{\natural}=\mathcal{F}_{Z^{\prime}}^{\natural}\left[d^{Z}\right]$ for each $Z^{\prime}$, and such that the pullbacks of $\iota_{Z^{\prime}}\left[d^{Z}\right]$ and $\iota_{Z^{\prime}}^{\prime}$ to $Z^{\prime} \times_{\bar{Z}} Z_{[\sigma]}^{\text {tor }}$, for some top-dimensional $\sigma \in \Sigma_{Z}^{+}$as in the proof of Lemma 2.3.16, coincide with each other.

Proof. As in the proof of Lemma 4.2.13, since the fibered category of perverse sheaves is a stack (see [6, 2.2.19]), it suffices to construct $\mathcal{G}$ and $\iota_{Z^{\prime}}^{\prime}$ over the étale boundary charts of $Z^{\text {tor }}$. Let $Z^{\prime}$ be any stratum of $\bar{Z}$. Suppose that we have already defined $\mathcal{G}$ over the largest open subscheme of $Z^{\text {tor }}$ containing all stratum $Z^{\prime \prime}$ of $\bar{Z}$ that contain $Z^{\prime}$ in its closure but differ from $Z^{\prime}$, and defined $\iota^{\prime} Z^{\prime \prime}$ and its extensions over some $\mathcal{U}_{Z^{\prime \prime}}^{\prime}$ as in Definition 4.1.3, for all such $Z^{\prime \prime}$. (When $Z^{\prime}=Z$, we simply have $\left.\mathcal{G}\right|_{Z}=\mathcal{F}_{Z}^{\natural}\left[d^{Z}\right]$, with $\iota_{Z}^{\prime}$ and its extensions being the identity morphisms.) 
Let $x$ be a point of a stratum $Z_{\left[\tau^{\prime \prime}\right]}^{\prime}$ of $Z_{[\sigma]}^{\text {tor }}$, which lies above a point $y$ of a stratum $Z_{\left[\tau^{\prime}\right]}^{\prime}$ of $Z^{\text {tor }}$ above $Z^{\prime}$. By using nested approximation as in the proof of [45, Proposition 4.3], there exist a torus homomorphism $E^{\prime \prime} \rightarrow E^{\prime}$ and an abelian scheme torsor $C^{\prime \prime} \rightarrow C^{\prime}$, and representatives $\tau^{\prime \prime}$ and $\tau^{\prime}$ of $\left[\tau^{\prime \prime}\right]$ and $\left[\tau^{\prime}\right]$, respectively, such that there exists a commutative diagram

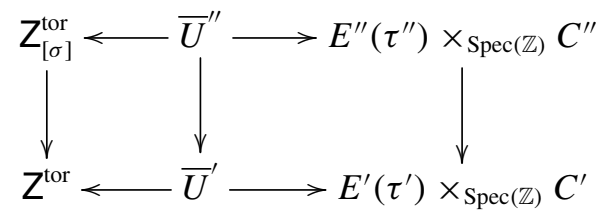

in which the squares are both Cartesian, and in which the horizontal morphisms define compatible étale neighborhoods of $x$ and $y$ such that the preimages of $Z$ and $E^{\prime} \times_{\mathrm{Spec}(\mathbb{Z})} C^{\prime}$ coincide as an open subscheme $U^{\prime}$ of $\bar{U}^{\prime}$; such that the preimages of $Z_{[\sigma]}, E^{\prime \prime} \times_{\operatorname{Spec}(\mathbb{Z})} C^{\prime \prime}$, and $U^{\prime}$ coincide as an open subscheme $U^{\prime \prime}$ of $\bar{U}^{\prime \prime}$; and such that the pullbacks of the horizontal étale morphisms under the canonical morphisms $Z^{\prime} \times_{\bar{Z}} Z^{\text {tor }} \rightarrow Z^{\text {tor }}$ and $E^{\prime}\left(\tau^{\prime}\right)^{+} \times_{\operatorname{Spec}(\mathbb{Z})} C^{\prime} \rightarrow E^{\prime}\left(\tau^{\prime}\right) \times_{\operatorname{Spec}(\mathbb{Z})} C^{\prime}$ (when defined) are open immersions. Up to replacing $\bar{U}^{\prime}$ and $\bar{U}^{\prime \prime}$ with open subschemes, we may assume that all étale morphisms above have connected geometric fibers. Then there exists an étale morphism $\bar{U}^{\prime \prime \prime} \rightarrow \bar{U}^{\prime \prime}$ with connected geometric fibers such that the pullback of $\iota_{Z}$ and $\iota_{Z^{\prime}}$ extend to isomorphisms

$$
\iota_{U^{\prime \prime \prime}}:\left(\mathrm{Y}_{U^{\prime \prime \prime}} \rightarrow \mathrm{Y}_{\Sigma}^{\text {tor }}\right)^{*} \mathcal{F} \stackrel{\sim}{\rightarrow}\left(\mathrm{Y}_{U^{\prime \prime \prime}} \rightarrow \mathrm{Y}_{Z}^{\natural}\right)^{*} \mathcal{F}_{Z}^{\natural}
$$

and

$$
\iota_{\bar{U}^{\prime \prime \prime}}:\left(\mathrm{Y}_{\bar{U}^{\prime \prime \prime}} \rightarrow \mathrm{Y}_{\Sigma}^{\mathrm{tor}}\right)^{*} \mathcal{F} \stackrel{\sim}{\rightarrow}\left(\mathrm{Y}_{\bar{U}^{\prime \prime \prime}} \rightarrow \mathrm{Y}_{\mathrm{Z}^{\prime}}^{\natural}\right)^{*} \mathcal{F}_{\mathrm{Z}^{\prime}}^{\natural}
$$

(see Definition 4.1.3), where $U^{\prime \prime \prime}$ denotes the preimage of $U^{\prime \prime}$ in $\bar{U}^{\prime \prime \prime}$, and where $\mathrm{Y}_{U^{\prime \prime \prime}}:=U^{\prime \prime \prime} \times_{\mathrm{X}_{\mathcal{H}, \Sigma}^{\text {tor }}} \mathrm{Y}_{\Sigma}^{\text {tor }}$ and $\mathrm{Y}_{\bar{U}^{\prime \prime \prime}}:=\bar{U}^{\prime \prime \prime} \times_{\mathrm{X}_{\mathcal{H}, \Sigma}^{\text {tor }}} \mathrm{Y}_{\Sigma}^{\text {tor }}$

Let $\mathcal{G}_{Z^{\prime}, U^{\prime}}^{\natural}$ denote the pullback of $\mathcal{G}_{Z^{\prime}}^{\natural}=\mathcal{F}_{Z^{\prime}}^{\natural}\left[d^{Z}\right]$ to $Y_{Z, U^{\prime}}:=U^{\prime} \times{ }_{Z^{\text {tor }}} Y_{Z}^{\text {tor }}$, which is a shifted perverse sheaf because $U^{\prime} \rightarrow Z^{\prime}$ is smooth of constant relative dimension. Since $\mathrm{Z}_{[\sigma]} \cong C \rightarrow \mathrm{Z}$ is smooth of constant relative dimension, the further pullback $\mathcal{G}_{Z^{\prime}, U^{\prime \prime \prime}}^{\natural}$ of $\mathcal{G}_{Z^{\prime}, U^{\prime}}^{\natural}$ to $Y_{U^{\prime \prime \prime}}$, which coincides with the pullback of $\mathcal{G}_{Z^{\prime}}^{\natural}$ under $Y_{U^{\prime \prime \prime}} \rightarrow Y_{Z^{\prime}}$, is also a shifted perverse sheaf. Using the isomorphisms $\iota_{U^{\prime \prime \prime}}$ and $\iota_{\bar{U}^{\prime \prime \prime}}$ above up to shifting, the pullback of $\mathcal{F}_{Z}^{\natural}\left[d^{Z}\right]$ under $Y_{U^{\prime \prime \prime}} \rightarrow Y_{Z}^{\natural}$ is isomorphic to $\mathcal{G}_{Z^{\prime}, U^{\prime \prime \prime}}^{\natural}$. Since $U^{\prime \prime \prime} \rightarrow U^{\prime}$ is smooth and has connected geometric fibers, by [6, 4.2.5 and 4.2.6.2], this uniquely descends to an isomorphism between $\mathcal{F}_{Z^{\prime}}^{\natural}\left[d^{Z}\right]$ and $\mathcal{G}_{Z^{\prime}, U^{\prime}}^{\natural}$ over the image of $Y_{U^{\prime \prime \prime}}$ in $Y_{Z^{\prime}}$. Hence, the pullback $\mathcal{G}_{Z^{\prime}, \bar{U}^{\prime}}^{\natural}$ of $\mathcal{G}_{Z^{\prime}}^{\natural}$ to $Y_{Z, \bar{U}^{\prime}}:=\bar{U}^{\prime} \times_{Z^{\text {tor }}} Y_{Z}^{\text {tor }}$, which necessarily has no support in $Y_{Z, \bar{U}^{\prime}}-Y_{Z, U^{\prime}}$, defines 
an extension of the partially defined $\mathcal{G}$ over the image of $\mathrm{Y}_{\bar{U}^{\prime \prime \prime}} \rightarrow \mathrm{Y}_{Z, \bar{U}^{\prime}}$. By varying $x$ (and $y$ ), we obtain étale neighborhoods which induce an open covering of the preimage of $Z^{\prime}$ in $Z^{\text {tor }}$, and hence by descent an extension of $\mathcal{G}$ over a larger open subscheme containing this preimage, with the desired $\iota_{Z^{\prime}}^{\prime}$ and its extensions over some collection $\overline{\mathcal{U}}_{Z^{\prime}}$ as in Definition 4.1.3. Thus, by inductively repeating this process until we exhaust all strata $Z^{\prime}$ of $\bar{Z}$, the lemma follows.

Now we are ready to state and prove our generalizations of Morel's results:

THEOREM 4.5.26. Suppose $\mathrm{T}=\operatorname{Spec}(k)$, where $k$ is a finite field of characteristic p. Let $\mathrm{Y}$ be a well-positioned subset of $\left(\mathrm{X}_{\mathcal{H}}\right)_{\mathrm{T}}$, with associated $\mathrm{Y}^{\natural}=\left\{\mathrm{Y}_{\mathrm{Z}}^{\natural}\right\}_{\mathrm{Z}}$ as in Definition 2.2.1, and with partial minimal and toroidal compactifications $\mathrm{Y}^{\mathrm{min}}$ and $\mathrm{Y}_{\Sigma}^{\mathrm{tor}}$ as in Definition 2.3.1 and Theorem 2.3.2.

Suppose that $V=V_{\xi}$ is an algebraic representation of $\mathrm{G} \otimes_{\mathbb{Z}} \mathbb{Q}$ on a finitedimensional vector space over $\overline{\mathbb{Q}}_{\ell}$ that is pure of weight $-a \in \mathbb{Z}$ (as in Definition 4.5.2), and that $\mathcal{F}$ is a well-positioned pure perverse sheaf of weight $b \in \mathbb{Z}$ over $Y_{\Sigma}^{\text {tor }}$, equipped with a collection $\mathcal{F}^{\natural}=\left\{\left(\mathcal{F}_{Z}^{\natural}, \iota_{Z}\right)\right\}_{Z}$ as in Definition 4.1.3 such that $\mathcal{F}_{Z}^{\natural}\left[-d^{Z}\right]$ is a pure perverse sheaf of weight $b-d^{Z}$ over $Y_{Z}^{\natural}$, where $d^{Z}:=\operatorname{dim}\left(\left(\mathbf{X}_{\mathcal{H}}\right)_{\mathrm{T}}\right)-\operatorname{dim}\left(Z_{\mathrm{T}}\right)$, for each $\mathbf{Z}$.

Let $\mathrm{Z}$ be a stratum of $\mathrm{X}_{\mathcal{H}}^{\min }$. Let $i^{\mathrm{min}}, i_{\mathrm{Y}}^{\min }$, and so forth be defined as in the paragraphs preceding Theorems 4.3 .10 and 4.3.16. Let $\mathcal{F}_{\mathrm{Y}}, \mathcal{V}_{\mathrm{Y}}$, and $\mathcal{V}_{\mathscr{C}, \mathrm{Y}_{\mathrm{Z}}}^{\natural, \leqslant a}$ denote the pullbacks of $\mathcal{F}, \mathcal{V}$, and $\mathcal{V}_{\mathscr{C}}^{\natural, \leqslant a}$ (see Definition 4.5.22) to $\mathrm{Y}, \mathrm{Y}$, and $\mathrm{Y}_{\mathrm{Z}}$, respectively. Then, under Assumptions 4.3.1 and 4.5.1, we have an equality

$$
\left[i_{\mathrm{Y}}^{\min , *} j_{\mathrm{Y}, ! *}^{\min }\left(\mathcal{V}_{\mathrm{Y}} \otimes^{L} \mathcal{F}_{\mathrm{Y}}\right)\right]=\sum_{\mathscr{C} \triangleright Z}(-1)^{r(\mathscr{C})-1}\left[\mathcal{V}_{\mathscr{C}, Y_{\mathrm{Z}}}^{\natural, \leqslant a} \otimes^{L} \mathcal{F}_{\mathrm{Z}}^{\natural}\right]
$$

in the Grothendieck group of $D_{c}^{b}\left(\mathrm{Y}_{\mathrm{Z}}, \overline{\mathbb{Q}}_{\ell}\right)$. (See Definitions 4.5.7, 4.5.12, and 4.5.22.)

Proof. We shall closely follow the arguments in [59, especially the proofs of 4.2.1 and 4.2.3]. Let $w_{\leqslant a+b}$ and $w_{>a+b}$ denote the weight truncation functors defined in [59, Section 3.1, page 36]. By Lemma 4.5.6, $\mathcal{V}_{Y} \otimes^{L} \mathcal{F}_{Y}$ is a pure perverse sheaf of weight $a+b$. Therefore, by [59, 3.1.4], there is a canonical isomorphism

$$
j_{\mathrm{Y}, ! *}^{\min }\left(\mathcal{V}_{\mathrm{Y}} \otimes^{L} \mathcal{F}_{\mathrm{Y}}\right) \stackrel{\sim}{\rightarrow} w_{\leqslant a+b} R j_{\mathrm{Y}, *}^{\min }\left(\mathcal{V}_{\mathrm{Y}} \otimes^{L} \mathcal{F}_{\mathrm{Y}}\right)
$$

in $D_{c}^{b}\left(\mathrm{Y}^{\min }, \overline{\mathbb{Q}}_{\ell}\right)$. For each $\mathscr{C}=\left\{\mathrm{Z}^{(c)}\right\}_{c}$ with indices ordered as

$$
c_{0}>c_{1}>\cdots>c_{r(\mathscr{C})}
$$


as in Lemma 4.5.8, and for each $0 \leqslant i \leqslant r(\mathscr{C})$, let $i_{\mathscr{C}}^{(i)}: \mathrm{Y}_{Z^{\left(c_{i}\right)}} \rightarrow \mathrm{Y}^{\min }$ denote the canonical morphism, so that $i_{\mathscr{C}}^{(0)}=j_{\mathrm{Y}}^{\min }$ and $i_{\mathscr{C}}^{(r(\mathscr{C}))}=i_{\mathrm{Y}}^{\min }$. By [59, 3.1.3 and 3.3.4; cf. 3.3.5], we have an equality

$$
\begin{aligned}
& {\left[i_{\mathrm{Y}}^{\min , *} w_{\leqslant a+b} R j_{\mathrm{Y}, *}^{\min }\left(\mathcal{V}_{\mathrm{Y}} \otimes^{L} \mathcal{F}_{\mathrm{Y}}\right)\right]=\sum_{\mathscr{C} \triangleright \mathrm{Z}}(-1)^{r(\mathscr{C})-1}\left[w_{\leqslant a+b} i_{\mathscr{C}}^{(r(\mathscr{C})), *} R i_{\mathscr{C}, *}^{(r(\mathscr{C})-1)}\right.} \\
& \left.w_{>a+b} i_{\mathscr{C}}^{(r(\mathscr{C})-1), *} \ldots R i_{\mathscr{C}, *}^{(2)} w_{>a+b} i_{\mathscr{C}}^{(2), *} R i_{\mathscr{C}, *}^{(1)} w_{>a+b} i_{\mathscr{C}}^{(1), *} R j_{\mathrm{Y}, *}^{\min }\left(\mathcal{V}_{\mathrm{Y}} \otimes^{L} \mathcal{F}_{\mathrm{Y}}\right)\right]
\end{aligned}
$$

in the Grothendieck group of $D_{c}^{b}\left(\mathrm{Y}_{\mathrm{Z}}, \overline{\mathbb{Q}}_{\ell}\right)$.

Let $\mathrm{Q}$ be as in Lemma 4.5.8, which is $\bigcap_{0 \leqslant i \leqslant r(\mathscr{C})} \mathrm{P}^{(i)}$ as in the paragraph following Lemma 4.5.14. For each $0 \leqslant i \leqslant r(\mathscr{C})$, let $\mathrm{U}^{(i)}, \mathrm{M}^{(i)}, \mathrm{G}_{l}^{(i)}$, and $\mathrm{G}_{h}^{(i)}$ be defined as in Definition 3.3.8 for $\mathrm{P}^{(i)}$, with $\Gamma_{\mathrm{P}^{(i)}} \subset \mathrm{G}_{l}^{(i)}(\mathbb{Q})$ defined by the corresponding $Z^{\left(c_{i}\right)}$ as in Definition 4.3.4, and let $\mathfrak{u}^{(i)}:=\operatorname{Lie} U^{(i)}\left(\mathbb{Q}_{\ell}\right)$. Let $\mathrm{Q}^{(i)}:=\bigcap_{0 \leqslant j \leqslant i} \mathrm{P}^{(j)}$, let $\mathrm{U}_{\mathrm{Q}^{(i)}}$ denote the unipotent radical of $\mathrm{Q}^{(i)}$, and let $\mathfrak{u}_{\mathrm{Q}^{(i)}}:=\operatorname{Lie} \mathrm{U}_{\mathrm{Q}^{(i)}}\left(\mathbb{Q}_{\ell}\right)$. Let $\mathrm{Q}_{l}^{(i)}:=\operatorname{ker}\left(\left(\mathrm{Q}^{(i)} / \mathrm{U}^{(i)}\right) \rightarrow \mathrm{G}_{h}^{(i)}\right)$, which is a parabolic subgroup of $\mathrm{G}_{l}^{(i)}$, with unipotent radical $\mathrm{U}_{\mathrm{Q}_{l}^{(i)}}:=\mathrm{U}_{\mathrm{Q}^{(i)}} / \mathrm{U}^{(i)}$ and Levi quotient $\mathrm{M}_{\mathrm{Q}_{l}^{(i)}}:=\mathrm{Q}_{l}^{(i)} / \mathrm{U}_{\mathrm{Q}_{l}^{(i)}}$ (cf. Lemma 4.5.8). Let $\Gamma_{\mathrm{Q}^{(i)}}$ be the image of $\Gamma_{\mathrm{P}^{(i)}} \cap \mathrm{Q}_{l}^{(i)}(\mathbb{Q})$ under the canonical homomorphism $\mathrm{Q}_{l}^{(i)}(\mathbb{Q}) \rightarrow \mathrm{M}_{\mathbf{Q}_{l}^{(i)}}(\mathbb{Q})$ (cf. Definition 4.5.12). When $i \geqslant 1$, also consider the canonical homomorphism $\mathrm{Q}^{(i)} / \mathrm{U}^{(i-1)} \rightarrow \mathrm{G}_{h}^{(i-1)}$, whose kernel is $\mathrm{Q}_{l}^{(i-1)}$, and whose image is a parabolic subgroup $\mathrm{P}_{h}^{(i)}$ of $\mathrm{G}_{h}^{(i-1)}$, with unipotent radical $\mathrm{U}_{h}^{(i)} \cong \mathrm{U}^{(i)} /\left(\mathrm{U}^{(i)} \cap \mathrm{U}^{(i-1)}\right) \cong \mathrm{U}_{\mathrm{Q}^{(i)}} / \mathrm{U}_{\mathrm{Q}^{(i-1)}}$ and Levi quotient $\mathrm{M}_{h}^{(i)} \cong \mathrm{G}_{h, l}^{(i)} \times \mathrm{G}_{h}^{(i)}$, where $\mathrm{G}_{h, l}^{(i)}:=\mathrm{M}_{\mathrm{Q}_{l}^{(i)}} / \mathrm{M}_{\mathrm{Q}_{l}^{(i-1)}}$. (Note that we have $\mathrm{U}^{(0)}=1$, $\mathrm{G}_{h}^{(0)}=\mathrm{G} \otimes_{\mathbb{Z}} \mathbb{Q}, \mathrm{Q}_{l}^{(0)}=1, \mathrm{U}_{\mathrm{Q}_{l}^{(0)}}=1$, and $\mathrm{M}_{\mathrm{Q}_{l}^{(0)}}=1$.) Let $\Gamma_{\mathrm{P}_{h}^{(i)}}$ be the image of $\Gamma_{\mathrm{Q}^{(i)}}$ under the canonical morphism $\mathrm{M}_{\mathrm{Q}_{l}^{(i)}} \rightarrow \mathrm{G}_{h, l}^{(i)}$, and let $\mathfrak{u}_{h}^{(i)}:=\operatorname{Lie}_{h}^{(i)}\left(\mathbb{Q}_{\ell}\right)$. By definition, we have $\Gamma_{\mathrm{P}_{h}^{(i)}} \cong \Gamma_{\mathrm{Q}^{(i)}} / \Gamma_{\mathrm{Q}^{(i-1)}}$ and $\mathfrak{u}_{h}^{(i)} \cong \mathfrak{u}_{\mathrm{Q}^{(i)}} / \mathfrak{u}_{\mathrm{Q}^{(i-1)}}$. We also have $\mathrm{M}_{\mathrm{Q}_{l}^{(i)}} \cong \prod_{1 \leqslant j \leqslant i} \mathrm{G}_{h, l}^{(j)}$ and $\mathrm{M}_{\mathrm{Q}_{l}} \cong \prod_{1 \leqslant i \leqslant r(\mathscr{C})} \mathrm{G}_{h, l}^{(i)}$.

Suppose that, for some $1 \leqslant i<r(\mathscr{C})$, we have a canonical isomorphism

$$
\begin{aligned}
& i_{\mathscr{C}}^{(i), *} R i_{\mathscr{C}, *}^{(i-1)} w_{>a+b} \cdots R i_{\mathscr{C}, *}^{(1)} w_{>a+b} i_{\mathscr{C}}^{(1), *} R i_{\mathscr{C}, *}^{(0)}\left(\mathcal{V}_{\mathrm{Y}} \otimes^{L} \mathcal{F}_{\mathrm{Y}}\right) \\
& \quad \cong \mathcal{V}_{\mathscr{C}, Y_{Z^{\left(c_{i}\right)}}^{\natural, \leqslant a \text {,re }}} \otimes^{L} \mathcal{F}_{Z^{\left(c_{i}\right)}}^{\natural}
\end{aligned}
$$

in $D_{c}^{b}\left(\mathrm{Y}_{Z^{\left(c_{i}\right)}}, \overline{\mathbb{Q}}_{\ell}\right)$, where $\mathcal{V}_{\mathscr{C}, \mathrm{Y}_{Z^{\left(c_{i}\right)}}^{\natural, \leqslant a} \text {, pre }}$ is associated with

$$
R \operatorname{Inv}\left(\Gamma_{\mathrm{Q}^{(i)}}, w_{<d_{\mathrm{Q},(i-1)}-a}^{\mathrm{Q}(i-1)} \cdots w_{<d_{\mathrm{Q},(1)}-a}^{\mathrm{Q},(1)}\left(R \operatorname{Inv}\left(\mathfrak{u}_{\mathrm{Q}^{(i)}},\left.V\right|_{\mathrm{Q}^{(i)}}\right)\right)\right)
$$

in $D^{b}\left(\mathrm{G}_{h}^{(i)} \otimes_{\mathbb{Z}} \overline{\mathbb{Q}}, \overline{\mathbb{Q}}_{\ell}\right)$ (cf. Definition 4.3.8 and (4.5.20)). When $i=1$, this is just Theorem 4.3.16 (with no truncation needed in (4.5.31)). 
Since $\mathcal{F}_{Z^{\left(c_{i}\right)}}^{\natural}\left[-d^{Z^{\left(c_{i}\right)}}\right]$ is a pure perverse sheaf of weight $b-d^{Z^{\left(c_{i}\right)}}$ over $Y_{Z^{\left(c_{i}\right)}}$, and since $d_{\mathrm{Q},(i)}=d_{\mathrm{Z}^{\left(c_{i}\right)}}$, by the analogue of Lemma 4.5.6 for $Z^{\left(c_{1}\right)}$, and by [59, the proof of 4.1.2, based on 2.1.4] (cf. Lemma 4.5.4), we have a canonical isomorphism

$$
w_{>a+b}\left(\mathcal{V}_{\mathscr{C}, \mathrm{Y}_{Z^{\left(c_{i}\right)}}^{\natural, \leqslant a, \text { pre }}}^{L} \otimes^{L} \mathcal{F}_{Z^{\left(c_{i}\right)}}^{\natural}\right) \cong \mathcal{V}_{\mathscr{C}, \mathrm{Y}_{Z^{\left(c_{i}\right)}}^{\natural, \leqslant a}}^{\otimes^{L}} \mathcal{F}_{\mathrm{Z}^{\left(c_{i}\right)}}^{\natural}
$$

in $D_{c}^{b}\left(\mathrm{Y}_{\mathrm{Z}^{\left(c_{i}\right)}}, \overline{\mathbb{Q}}_{\ell}\right)$, where $\mathcal{V}_{\mathscr{C}, \mathrm{Y}_{\mathrm{Z}^{\left(c_{1}\right)}}^{\natural, \leqslant a}}$ is associated with

$$
R \operatorname{Inv}\left(\Gamma_{\mathrm{Q}^{(i)}}, w_{<d_{\mathrm{Q},(i)}-a}^{\mathrm{Q},(i)} \cdots w_{<d_{\mathrm{Q},(1)}-a}^{\mathrm{Q},(1)}\left(R \operatorname{Inv}\left(\mathfrak{u}_{\mathrm{Q}^{(i)}},\left.V\right|_{\mathrm{Q}^{(i)}}\right)\right)\right)
$$

in $D^{b}\left(\mathrm{G}_{h}^{(i)} \otimes_{\mathbb{Z}} \overline{\mathbb{Q}}, \overline{\mathbb{Q}}_{\ell}\right)$.

Since $\Gamma_{\mathrm{P}_{h}^{(i+1)}} \cong \Gamma_{\mathrm{Q}^{(i+1)}} / \Gamma_{\mathrm{Q}^{(i)}}$, since $\Gamma_{\mathrm{Q}^{(i)}} \subset \mathrm{M}_{\mathrm{Q}_{l}^{(i)}}(\mathbb{Q})$, since $\mathrm{M}_{\mathrm{Q}_{l}^{(i)}}$ commutes with $\mathrm{G}_{h}^{(i)}$ and hence also with $\mathrm{U}_{h}^{(i+1)}$, and since the cocharacters $s^{\mathrm{Q},(1)}, \ldots, s^{\mathrm{Q},(i)}$ act trivially on $\mathfrak{u}_{h}^{(i+1)} \cong \mathfrak{u}_{\mathrm{Q}^{(i+1)}} / \mathfrak{u}_{\mathrm{Q}^{(i)}}$, we have a canonical isomorphism

$$
\begin{aligned}
& R \operatorname{Inv}\left(\Gamma_{\mathrm{P}_{h}^{(i+1)}}, w_{<d_{\mathrm{Q},(i)}-a}^{\mathrm{Q},(i)} R \operatorname{Inv}\left(\mathfrak{u}_{h}^{(i+1)},\right.\right. \\
& \left.\left.\left.\left(R \operatorname{Inv}\left(\Gamma_{\mathrm{Q}^{(i)}}, w_{<d_{\mathrm{Q},(i-1)}-a}^{\mathrm{Q},(i)} \cdots w_{<d_{\mathrm{Q},(1)}-a}^{\mathrm{Q},(1)}\left(R \operatorname{Inv}\left(\mathfrak{u}_{\mathrm{Q}^{(i)}},\left.V\right|_{\mathrm{Q}^{(i)}}\right)\right)\right)\right)\right|_{\mathrm{P}_{h}^{(i+1)}}\right)\right) \\
& \quad \cong R \operatorname{Inv}\left(\Gamma_{\mathrm{Q}^{(i+1)}}, w_{<d_{\mathrm{Q},(i)}-a}^{\mathrm{Q},(i)} \cdots w_{<d_{\mathrm{Q},(1)}-a}^{\mathrm{Q},(1)}\left(R \operatorname{Inv}\left(\mathfrak{u}_{\mathrm{Q}^{(i+1)}},\left.V\right|_{\mathrm{Q}^{(i+1)}}\right)\right)\right) .
\end{aligned}
$$

By Lemmas 2.3.16 and 4.5.24, and by applying Theorem 4.3.16 to the pullback to $\mathrm{Y}_{Z^{\left(c_{i+1}\right)}}$ of $R\left(\mathrm{Y}_{Z^{\left(c_{i}\right)}} \hookrightarrow \mathrm{Y}_{Z^{\left(c_{i}\right)}}^{\min }\right)_{*}\left(\mathcal{V}_{\mathscr{C}, \mathrm{Y}^{\left(c_{i}\right)}}^{\natural, a a} \otimes^{L} \mathcal{F}_{Z^{\left(c_{i}\right)}}^{\natural}\right)$, we obtain (4.5.30) with $i$ replaced with $i+1$. In the final step, when $i+1=r(\mathscr{C})$, so that $\mathrm{Z}^{\left(c_{r(\mathscr{C})}\right)}=\mathrm{Z}$, the same argument for showing (4.5.32) gives a canonical isomorphism

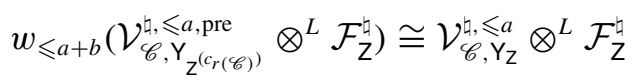

in $D_{c}^{b}\left(\mathrm{Y}_{\mathrm{Z}}, \overline{\mathbb{Q}}_{\ell}\right)$, and hence we obtain a canonical isomorphism

$$
\begin{aligned}
& w_{\leqslant a+b} i_{\mathscr{C}}^{(r(\mathscr{C})), *} R i_{\mathscr{C}, *}^{(r(\mathscr{C})-1)} w_{>a+b} \cdots R i_{\mathscr{C}, *}^{(1)} w_{>a+b} i_{\mathscr{C}}^{(1), *} R i_{\mathscr{C}, *}^{(0)}\left(\mathcal{V}_{Y} \otimes^{L} \mathcal{F}_{Y}\right) \\
& \simeq \mathcal{V}_{\mathscr{C}, Y_{Z}}^{\natural, \leqslant a} \otimes^{L} \mathcal{F}_{Z}^{\natural} .
\end{aligned}
$$

in $D_{c}^{b}\left(\mathrm{Y}_{\mathrm{Z}}, \overline{\mathbb{Q}}_{\ell}\right)$. Now, just combine (4.5.28), (4.5.29), and (4.5.36).

THEOREM 4.5.37. Suppose that $V=V_{\xi}$ and $-a \in \mathbb{Z}$ are as in Theorem 4.5.26. Let $d:=\operatorname{dim}\left(\left(\mathrm{X}_{\mathcal{H}}\right)_{\eta}\right)$. Consider the nearby cycle functors $R \Psi_{\mathrm{X}_{\mathcal{H}}}$, and others, defined by some compatible choices of geometric points $\bar{\eta}$ and $\bar{s}$ above the generic and special points $\eta$ and $s$ of S, respectively, as in [45, Section 5.1], where 
$\mathrm{S}=\operatorname{Spec}\left(\mathcal{O}_{K}\right)$, and where $K$ is the $v$-adic completion of $F_{0}$ at some place $v \mid p$, as in Section 4.4. Then, under Assumptions 4.3.1 and 4.5.1, we have an equality

$$
\left[i_{\bar{s}}^{\min , *} j_{\bar{s}_{, ! *}}^{\min } R \Psi_{\mathrm{X}_{\mathcal{H}}}(\mathcal{V}[d])\right]=\sum_{\mathscr{C} \triangleright Z}(-1)^{r(\mathscr{C})-1}\left[\mathcal{V}_{\mathscr{C}, Z_{\bar{s}}^{\natural, ~}} \otimes^{L} R \Psi_{Z}\left(\overline{\mathbb{Q}}_{\ell}\right)[d]\right]
$$

in the Grothendieck group of $D_{c}^{b}\left(\mathrm{Z}_{\bar{s}} \times \bar{\eta}, \overline{\mathbb{Q}}_{\ell}\right)$.

Proof. We shall closely follow [81], with additional inputs from [62] and [45, Section 5], and from this article. Since the argument is global, let us consider $\mathrm{M}_{\mathcal{H}}$, $\mathrm{M}_{\mathcal{H}}^{\min }$, and so forth over $\operatorname{Spec}\left(F_{0}\right)$ as in Assumption 2.1.1. Note that these are the generic fibers for the analogues of $X_{\mathcal{H}}, X_{\mathcal{H}}^{\min }$, and so forth, with the base scheme $S$ replaced with some analogue over $\operatorname{Spec}\left(\mathcal{O}_{F_{0},(q)}\right)$ for any good prime $q \neq p$ for $\left(\mathcal{O}, \star, L,\langle\cdot, \cdot\rangle, h_{0}\right)$ and $\mathcal{H}$ (in the sense that $\mathcal{H}$ is maximal hyperspecial at $q$ ).

Consider the categories $\mathcal{M}\left(\mathrm{M}_{\mathcal{H}}\right), \mathcal{M}\left(\mathrm{M}_{\mathcal{H}}^{\min }\right)$, and so forth of horizontal perverse sheaves which admit weight filtrations, as in $[62,6.1]$. For $V=V_{\xi}$ as in Theorem 4.5.26, the associated sheaf $\mathcal{V}[d]$ over $\mathrm{M}_{\mathcal{H}}$ (which can be defined using any analogues of $\mathrm{X}_{\mathcal{H}}$ as above over $\operatorname{Spec}\left(\mathcal{O}_{F_{0},(q)}\right)$ for some good $\left.q \neq p\right)$ is an object in $\mathcal{M}\left(\mathrm{X}_{\mathcal{H}}\right)$, and it follows from $[62,8.1 .4]$ and the same argument of the proof of Theorem 4.5 .26 (with $\mathcal{F}$ and $\mathcal{F}_{Z}^{\natural}$ being $\overline{\mathbb{Q}}_{\ell}[d]$ for each Z) that, after pullback from $\operatorname{Spec}\left(F_{0}\right)$ to $\eta=\operatorname{Spec}(K)$, we have an equality

$$
\left[i_{\eta}^{\min , *} j_{\eta, ! *}^{\min }(\mathcal{V}[d])\right]=\sum_{\mathscr{C} \triangleright Z}(-1)^{r(\mathscr{C})-1}\left[\mathcal{V}_{\mathscr{C}, Z_{\eta}}^{\natural, \leqslant a}[d]\right]
$$

in the Grothendieck group of $D_{c}^{b}\left(Z_{\eta}, \overline{\mathbb{Q}}_{\ell}\right)$, and hence also an equality

$$
\left[j_{\eta, ! *}^{\min }(\mathcal{V}[d])\right]=\sum_{Z^{\prime}} \sum_{\mathscr{C}^{\prime} \triangleright Z^{\prime}}(-1)^{r\left(\mathscr{C}^{\prime}\right)-1}\left[i_{\eta, !}^{\prime, \min } \mathcal{V}_{\mathscr{C}^{\prime}, Z_{\eta}^{\prime}}^{\natural, \leqslant a}[d]\right]
$$

in the Grothendieck group of $D_{c}^{b}\left(\left(\mathrm{X}_{\mathcal{H}}^{\mathrm{min}}\right)_{\eta}, \overline{\mathbb{Q}}_{\ell}\right)$, where the objects associated with $Z^{\prime}$ are denoted with an additional prime.

By [45, Theorem 5.26], we have a canonical isomorphism

$$
R \Psi_{\mathrm{X}_{\mathcal{H}}} j_{\eta, ! *}^{\min }(\mathcal{V}[d]) \stackrel{\sim}{\rightarrow} j_{\bar{s}, ! *}^{\min } R \Psi_{\mathrm{X}_{\mathcal{H}}}(\mathcal{V}[d])
$$

in $D_{c}^{b}\left(\left(\mathbf{X}_{\mathcal{H}}^{\min }\right)_{\bar{s}} \times \bar{\eta}, \overline{\mathbb{Q}}_{\ell}\right)$. For each $\mathbf{Z}^{\prime}$, by decomposing the canonical morphism $i^{\prime \text {,min }}: Z^{\prime} \rightarrow X_{\mathcal{H}}^{\min }$ as a composition of the open immersion $Z^{\prime} \hookrightarrow Z^{\prime \text {,min }}$ and the closed immersion $Z^{\prime}$,min $\rightarrow X_{\mathcal{H}}^{\text {min }}$ using Lemma 2.3.16, by applying [45, Theorem 5.23] to $Z^{\prime} \hookrightarrow Z^{\prime \prime \text { min }}$, and by the proper base change theorem (cf. [3, XII, 5.1] and [16, XIII, (2.1.7.1)]), we have a canonical isomorphism

$$
i_{\bar{s}, !}^{\prime, \min } R \Psi_{\mathrm{Z}^{\prime}}\left(\mathcal{V}_{\mathscr{C}^{\prime}, \mathrm{Z}_{\eta}^{\prime}}^{\natural, \leqslant a}\right) \stackrel{\sim}{\rightarrow} R \Psi_{\mathrm{X}_{\mathcal{H}}^{\min }}\left(i_{\eta, !}^{\prime, \min } \mathcal{V}_{\mathscr{C}^{\prime}, \mathrm{Z}_{\eta}^{\prime}}^{\natural, \leqslant a}\right)
$$


in $D_{c}^{b}\left(\left(\mathbf{X}_{\mathcal{H}}^{\min }\right)_{\bar{s}} \times \bar{\eta}, \overline{\mathbb{Q}}_{\ell}\right)$. On the other hand, since $\mathcal{V}_{\mathscr{C}^{\prime}, Z_{\eta}^{\prime}}^{\natural, \leqslant a}$ is quasi-isomorphic to a direct sum of shifted lisse sheaves, we have a canonical isomorphism

$$
\mathcal{V}_{\mathscr{C}^{\prime}, Z_{\bar{s}}}^{\natural, a a} \otimes^{L} R \Psi_{Z^{\prime}}\left(\overline{\mathbb{Q}}_{\ell}\right) \stackrel{\sim}{\rightarrow} R \Psi_{Z^{\prime}}\left(\mathcal{V}_{\mathscr{C}^{\prime}, Z_{\eta}^{\prime}}^{\natural, \leqslant a}\right)
$$

in $D_{c}^{b}\left(Z_{\bar{s}}^{\prime} \times \bar{\eta}, \overline{\mathbb{Q}}_{\ell}\right)$ (as we have seen in Section 4.4). Thus, we obtain the equality

$$
\left[j_{\bar{s}, ! *}^{\min } R \Psi_{\mathrm{X}_{\mathcal{H}}}(\mathcal{V}[d])\right]=\sum_{Z^{\prime}} \sum_{\mathscr{C}^{\prime} \triangleright Z^{\prime}}(-1)^{r\left(\mathscr{C}^{\prime}\right)-1}\left[i_{\bar{s}, !}^{\prime, \min }\left(\mathcal{V}_{\mathscr{C}^{\prime}, Z_{\bar{s}}^{\prime}}^{\natural, \leqslant a} \otimes^{L} R \Psi_{Z^{\prime}}\left(\overline{\mathbb{Q}}_{\ell}\right)[d]\right)\right]
$$

in the Grothendieck group of $D_{c}^{b}\left(\left(\mathrm{X}_{\mathcal{H}}^{\min }\right)_{\bar{s}} \times \bar{\eta}, \overline{\mathbb{Q}}_{\ell}\right)$ by combining (4.5.39), (4.5.41), (4.5.42), and (4.5.43). By applying $i_{\bar{s}}^{\min , *}$ to (4.5.44), we also obtain the equality (4.5.38) in the Grothendieck group of $D_{c}^{b}\left(Z_{\bar{s}} \times \bar{\eta}, \overline{\mathbb{Q}}_{\ell}\right)$, as desired.

REMARK 4.5.45. In order to establish (4.5.39) and (4.5.40), instead of resorting to results in [62], we may proceed as in the proof of [82, 1.4.8] and resort to Laumon's Cebotarev density theorem for perverse sheaves as in $[47,1.1 .2]$ (or rather an analogue of it), by applying Theorem 4.5.26 to $\mathrm{Y}=\left(\mathrm{X}_{\mathcal{H}}\right)_{s}$ and $\mathcal{F}=\Lambda_{\left(X_{\mathcal{H}, \Sigma}^{\text {tor })_{s}}\right.}[d]$ (for some smooth $\Sigma$ ), after replacing $\mathrm{X}_{\mathcal{H}}$, and others, with their analogues over $\operatorname{Spec}\left(\mathcal{O}_{F_{0},(q)}\right)$, for all good primes $q \nmid p \ell$.

COROLlaRY 4.5.46. With the same setting as in Theorem 4.5.37, the perverse sheaf $j_{\bar{s} ! * *}^{\min }\left(R \Psi_{\mathrm{X}_{\mathcal{H}}}(\mathcal{V}[d])\right)$ has no support contained in the boundary of $\left(\mathrm{X}_{\mathcal{H}}^{\min }\right)_{\bar{s}}$.

Proof. As in the case of (4.5.43), we have a canonical isomorphism

$$
\mathcal{V} \otimes{ }^{L} R \Psi_{\mathrm{X}_{\mathcal{H}}}\left(\overline{\mathbb{Q}}_{\ell}[d]\right) \stackrel{\sim}{\rightarrow} R \Psi_{\mathrm{X}_{\mathcal{H}}}(\mathcal{V}[d])
$$

in $D_{c}^{b}\left(\left(\mathbf{X}_{\mathcal{H}}\right)_{\bar{s}} \times \bar{\eta}, \overline{\mathbb{Q}}_{\ell}\right)$. Since Assumption 4.5.1 implies Assumption 3.7.7, by Lemma 3.7.9, Proposition 3.7.13, and Lemma 4.2.23, we have the following facts: The pure isotypic subquotients of $R \Psi_{\mathrm{X}_{\mathcal{H}}}\left(\overline{\mathbb{Q}}_{\ell}[d]\right)$ extend to well-positioned perverse sheaves $\mathcal{F}_{\alpha}$ over $\left(\mathrm{X}_{\mathcal{H}, \Sigma}^{\text {tor }}\right)_{\bar{s}}$, labeled by some index $\alpha$. Each such $\mathcal{F}_{\alpha}$ is equipped with a collection $\mathcal{F}_{\alpha}^{\natural}=\left\{\left(\mathcal{F}_{\alpha, \mathrm{Z}}^{\natural}, \iota_{\alpha, \mathrm{Z}}\right)\right\}_{\mathrm{Z}}$, where $\mathcal{F}_{\alpha, \mathrm{Z}}^{\natural}\left[-d^{\mathrm{Z}}\right]$ is a pure isotypic subquotient of $R \Psi_{Z}\left(\overline{\mathbb{Q}}_{\ell}\left[d_{Z}\right]\right)$, with $d_{Z}:=\operatorname{dim}\left(Z_{\eta}\right)$ and $d^{Z}:=d-d_{Z}$ as usual, for each Z. Since $\mathcal{F}_{\alpha}$ and $\mathcal{F}_{\alpha, Z}^{\natural}$ have isomorphic pullbacks to schemes $U$ smooth over $Z$ of relative dimension $d^{Z}$, they have the same weight. Moreover, pure isotypic subquotients of $R \Psi_{\mathrm{Z}}\left(\overline{\mathbb{Q}}_{\ell}\left[d_{\mathrm{Z}}\right]\right)$ are exactly such $\mathcal{F}_{\alpha, \mathrm{Z}}^{\natural}\left[-d^{\mathrm{Z}}\right]$, over all $\alpha$. By applying Theorem 4.5.26 to such $\mathcal{F}_{\alpha}$ and $\mathcal{F}_{\alpha, Z}^{\natural}$, by summing up identities like (4.5.27) over all $Z$ and $\alpha$ (which have the same form regardless of the possibly 
varying weights of $\mathcal{F}_{\alpha}$ ), and by (4.5.44) and (4.5.47), we obtain an identity

$$
\left[j_{\bar{s}, ! *}^{\min }\left(R \Psi_{\mathrm{X}_{\mathcal{H}}}(\mathcal{V}[d])\right)\right]=\sum_{\alpha}\left[j_{\bar{s}, ! *}^{\min }\left(\mathcal{V} \otimes^{L}\left(\left.\mathcal{F}_{\alpha}\right|_{\left(\mathrm{X}_{\mathcal{H}}\right)_{s}}\right)\right)\right]
$$

in the Grothendieck group of $D_{c}^{b}\left(\left(\mathrm{X}_{\mathcal{H}}^{\min }\right)_{\bar{s}} \times \bar{\eta}, \overline{\mathbb{Q}}_{\ell}\right)$. For each $\alpha$, since $\mathcal{V}$ is quasi-isomorphic to a direct sum of shifted lisse sheaves, and since $\mathcal{F}_{\alpha}$ is the middle perversity extension of a shifted (pure isotypic) lisse étale sheaf over an irreducible smooth scheme, $j_{! *}^{\min }\left(\mathcal{V} \otimes^{L}\left(\left.\mathcal{F}_{\alpha}\right|_{\left(X_{\mathcal{H}}\right)_{s}}\right)\right)$ has no support contained in the boundary of $\left(\mathrm{X}_{\mathcal{H}}^{\min }\right)_{\bar{s}}$. Thus, the corollary follows from (4.5.48), as desired.

\section{Acknowledgments}

The first author is partially supported by the National Science Foundation under agreement no. DMS-1352216, by an Alfred P. Sloan Research Fellowship, by the Université Paris 13, and by the University of Minnesota. The second author is partially supported by the A.N.R. (Agence Nationale de la Recherche) under the program ANR-14-CE25-0002.

\section{References}

[1] F. Andreatta, A. Iovita and V. Pilloni, ' $p$-adic families of Siegel modular cuspforms', Ann. of Math. (2) 181(2) (2015), 623-697.

[2] M. Artin, 'Algebraic approximation of structures over complete local rings', Publ. Math. Inst. Hautes Études Sci. 36 (1969), 23-58.

[3] M. Artin, A. Grothendieck and J.-L. Verdier (Eds.), Théorie des topos et cohomologie étale des schémas (SGA 4), Tome 3, Lecture Notes in Mathematics, 305 (Springer, Berlin, Heidelberg, New York, 1973).

[4] A. Ash, D. Mumford, M. Rapoport and Y. Tai, Smooth Compactification of Locally Symmetric Varieties, 2nd edn, Cambridge Mathematical Library (Cambridge University Press, Cambridge, New York, 2010).

[5] W. L. Baily Jr. and A. Borel, 'Compactification of arithmetic quotients of bounded symmetric domains', Ann. of Math. (2) 84(3) (1966), 442-528.

[6] A. Beilinson, J. Bernstein, P. Deligne and O. Gabber, Faisceaux Pervers, 2nd edn, Astérisque, 100 (Société Mathématique de France, Paris, 2018).

[7] P. Berthelot, L. Breen and W. Messing, Théorie de Dieudonné cristalline II, Lecture Notes in Mathematics, 930 (Springer, Berlin, Heidelberg, New York, 1982).

[8] A. Borel and W. Casselman (Eds.), 'Automorphic forms, representations and $L$-functions', in Proceedings of Symposia in Pure Mathematics, Vol. 33, Part 2, held at Oregon State University, Corvallis, OR, July 11-August 5, 1977 (American Mathematical Society, Providence, Rhode Island, 1979).

[9] A. Borel and L. Ji, Compactifications of Symmetric and Locally Symmetric Spaces, Mathematics: Theory and Applications (Birkhäuser, Boston, 2006).

[10] S. Bosch, W. Lütkebohmert and M. Raybaud, Néron Models, Ergebnisse der Mathematik und ihrer Grenzgebiete, 3. Folge, 21 (Springer, Berlin, Heidelberg, New York, 1990). 
[11] J.-B. Bost, P. Boyer, A. Genestier, L. Lafforgue, S. Lysenko, S. Morel and B. C. Ngô (Eds.), De la géometrie algébrique aux formes automorphes (II): Une collection d'articles en l'honneur du soixantième anniversaire de Gérard Laumon, Astérisque, 370 (Société Mathématique de France, Paris, 2015).

[12] G. A. Boxer, 'Torsion in the coherent cohomology of Shimura varieties and Galois representations', PhD Thesis, Harvard University, Cambridge, Massachusetts, 2015.

[13] P. Cartier, L. Illusie, N. M. Katz, G. Laumon, Y. Manin and K. A. Ribet (Eds.), The Grothendieck festschrift: A Collection of Articles Written in Honer of the 60th Birthday of Alexander Grothendieck, Vol. 2 (Birkhäuser, Boston, 1990).

[14] P. Deligne, Variétés de Shimura: Interprétation modulaire, et techniques de construction de modèles canoniques, in Borel and Casselman [8], 247-290.

[15] P. Deligne, 'La conjecture de Weil. II', Publ. Math. Inst. Hautes Études Sci. 52 (1980), 137-252.

[16] P. Deligne and N. Katz (Eds.), Groupes de monodromie en géométrie algébrique (SGA 7 II), Lecture Notes in Mathematics, 340 (Springer, Berlin, Heidelberg, New York, 1973).

[17] T. Ekedahl, On the Adic Formalism, in Cartier et al. [13], 197-218.

[18] C. Faber, G. van der Geer and F. Oort (Eds.), Moduli of Abelian Varieties, Progress in Mathematics, 195 (Birkhäuser, Boston, 2001).

[19] G. Faltings and C.-L. Chai, Degeneration of Abelian Varieties, Ergebnisse der Mathematik und ihrer Grenzgebiete, 3. Folge, 22 (Springer, Berlin, Heidelberg, New York, 1990).

[20] U. Görtz and T. J. Haines, 'The Jordan-Hölder series for nearby cycles on some Shimura varieties and affine flag varieties', J. Reine Angew. Math. 609 (2007), 161-213.

[21] A. Grothendieck and J. Dieudonné, Eléments de géométrie algébrique, Publications Mathématiques de l'I.H.E.S., 4, 8, 11, 17, 20, 24, 28, 32 (Institut des Hautes Etudes Scientifiques, Paris, 1960, 1961, 1961, 1963, 1964, 1965, 1966, 1967).

[22] P. Hamacher, 'The $p$-rank stratification on the Siegel moduli space with Iwahori level structure', Manuscripta Math. 143(1-2) (2014), 51-80.

[23] P. Hamacher, 'The geometry of Newton strata in the reduction moduli $p$ of Shimura varieties of PEL type', Duke Math. J. 164(15) (2015), 2809-2895.

[24] M. Harris, K.-W. Lan, R. Taylor and J. Thorne, 'On the rigid cohomology of certain Shimura varieties', Res. Math. Sci. 3 (2016), article no. 37, 308 pp.

[25] P. Hartwig, 'Kottwitz-Rapoport and $p$-rank strata in the reduction of Shimura varieties of PEL type', Ann. Inst. Fourier (Grenoble) 65(3) (2015), 1031-1103.

[26] X. He and M. Rapoport, 'Stratifications in the reduction of Shimura varieties', Manuscripta Math. 152(3-4) (2018), 317-343.

[27] L. Illusie, Autour du théorème de monodromie locale, Périodes p-adiques (Bures-sur-Yvette, 1988), Astérisque 223 (Société Mathématique de France, Paris, 1994), 9-57.

[28] International Congress of Mathematicians, August 19-27, 2010, Hyderabad, India, Proceedings of the International Congress of Mathematicians, 2, Hindustan Book Agency, New Delhi; distributed by World Scientific, Singapore, 2010.

[29] R. Kiehl and R. Weissauer, Weil Conjectures, Perverse Sheaves, and l'adic Fourier Transform, Ergebnisse der Mathematik und ihrer Grenzgebiete, 3. Folge, 42 (Springer, Berlin, Heidelberg, New York, 2001).

[30] N. Koblitz, ' $p$-adic variation of the zeta-function over families of varieties defined over finite fields', Compos. Math. 31(2) (1975), 119-218.

[31] R. E. Kottwitz, 'Isocrystals with additional structure', Compos. Math. 56(2) (1985), 201-220.

[32] R. E. Kottwitz, 'Points on some Shimura varieties over finite fields', J. Amer. Math. Soc. 5(2) (1992), 373-444. 
[33] R. E. Kottwitz, 'Isocrystals with additional structure. II', Compos. Math. 109 (1997), 255-339.

[34] K.-W. Lan, 'Comparison between analytic and algebraic constructions of toroidal compactifications of PEL-type Shimura varieties', J. Reine Angew. Math. 664 (2012), 163-228.

[35] K.-W. Lan, 'Toroidal compactifications of PEL-type Kuga families', Algebra Number Theory 6(5) (2012), 885-966.

[36] K.-W. Lan, Arithmetic Compactification of PEL-type Shimura Varieties, London Mathematical Society Monographs, 36 (Princeton University Press, Princeton, 2013), errata and revision available online at the author's website.

[37] K.-W. Lan, 'Boundary strata of connected components in positive characteristics', Algebra Number Theory 9(9) (2015), 2035-2054, an appendix to the article 'Families of nearly ordinary Eisenstein series on unitary groups' by Xin Wan.

[38] K.-W. Lan, 'Compactifications of PEL-type Shimura varieties in ramified characteristics', Forum Math. Sigma 4 (2016), e1, 98 pp.

[39] K.-W. Lan, 'Higher Koecher's principle', Math. Res. Lett. 23(1) (2016), 163-199.

[40] K.-W. Lan, 'Vanishing theorems for coherent automorphic cohomology', Res. Math. Sci. 3 (2016), article no. 39, 43 pp.

[41] K.-W. Lan, 'Integral models of toroidal compactifications with projective cone decompositions', Int. Math. Res. Not. IMRN 2017(11) (2017), 3237-3280.

[42] K.-W. Lan, Compactifications of PEL-type Shimura Varieties and Kuga Families with Ordinary Loci (World Scientific, Singapore, 2018).

[43] K.-W. Lan, 'Compactifications of splitting models of PEL-type Shimura varieties', Trans. Amer. Math. Soc. 370(4) (2018), 2463-2515.

[44] K.-W. Lan and B. Stroh, 'Relative cohomology of cuspidal forms on PEL-type Shimura varieties', Algebra Number Theory 8(8) (2014), 1787-1799.

[45] K.-W. Lan and B. Stroh, 'Nearby cycles of automorphic étale sheaves', Compos. Math. 154(1) (2018), 80-119.

[46] K.-W. Lan and B. Stroh, Nearby Cycles of Automorphic étale Sheaves, II, Cohomology of Arithmetic Groups: On the Occasion of Joachim Schwermer's 66th Birthday, Bonn, Germany, June 2016 (eds. J. Cogdell, G. Harder, S. Kudla and F. Shahidi), Springer Proceedings in Mathematics \& Statistics, 245 (Springer International Publishing, 2018), 83-106.

[47] G. Laumon, 'Transformation de Fourier, constantes d'équations fonctionnelles et conjecture de Weil', Publ. Math. Inst. Hautes Études Sci. 65 (1987), 131-210.

[48] D. U. Lee, 'Non-emptiness of Newton strata of Shimura varieties of Hodge type', Algebra Number Theory 12(2) (2018), 259-283.

[49] Y. Liu and W. Zheng, 'Enhanced adic formalism and perverse $t$-structures for higher Artin stacks'. Preprint, 2012.

[50] K. Madapusi Pera, 'Toroidal compactifications of integral models of Shimura varieties of Hodge type'. Preprint, 2018.

[51] E. Mantovan, 'On the cohomology of certain PEL-type Shimura varieties', Duke Math. J. 129(3) (2005), 573-610.

[52] E. Mantovan, ' $\ell$-adic étale cohomology of PEL type Shimura varieties with non-trivial coefficients', in WIN-Woman in Numbers, Fields Institute Communications, 60 (American Mathematical Society, Providence, Rhode Island, 2011), 61-83.

[53] B. Mazur and W. Messing, Universal Extensions and One Dimensional Crystalline Cohomology, Lecture Notes in Mathematics, 370 (Springer, Berlin, Heidelberg, New York, 1974). 
[54] W. Messing, The Crystals Associated to Barsotti-Tate Groups: With Applications to Abelian Schemes, Lecture Notes in Mathematics, 264 (Springer, Berlin, Heidelberg, New York, 1972).

[55] B. Moonen, Group Schemes with Additional Structures and Weyl Group Cosets, in Faber et al. [18], 255-298.

[56] B. Moonen, 'A dimension formula for Ekedahl-Oort strata', Ann. Inst. Fourier (Grenoble) 54(3) (2004), 666-698.

[57] B. Moonen and T. Wedhorn, 'Discrete invariants of varieties in positive characteristic', Int. Math. Res. Not. IMRN 2004(72) (2004), 3855-3903.

[58] S. Morel, 'Complexes d'intersection des compactifications de Baily-Borel. Le cas des groupes unitaires sur $\mathbb{Q}^{\prime}$, PhD Thesis, Université Paris-Sud, Orsay, France, 2005.

[59] S. Morel, 'Complexes pondérés sur les compactifications de Baily-Borel: le cas des variétés de Siegel', J. Amer. Math. Soc. 21(1) (2008), 23-61.

[60] S. Morel, 'The intersection complex as a weight truncation and an application to Shimura varieties', in Proceedings of the International Congress of Mathematicians [28], 312-334.

[61] S. Morel, On the Cohomology of Certain Non-compact Shimura Varieties, Annals of Mathematics Studies, 173 (Princeton University Press, Princeton, 2010).

[62] S. Morel, 'Complexes mixtes sur un schéma de type fini sur $\mathbb{Q}$ '. Preprint, 2012.

[63] D. Mumford, Abelian Varieties, Tata Institute of Fundamental Research Studies in Mathematics, 5 (Oxford University Press, Oxford, 1970), with appendices by C. P. Ramanujam and Yuri Manin.

[64] B. C. Ngô and A. Genestier, 'Alcôves et p-rang des variétés abéliennes', Ann. Inst. Fourier (Grenoble) 52(6) (2002), 1665-1680.

[65] F. Oort, A Stratification of a Moduli Space of Abelian Varieties, in Faber et al. [18], $345-416$.

[66] F. Oort, 'Foliations in moduli spaces of abelian varieties', J. Amer. Math. Soc. 17(2) (2004), 267-296.

[67] G. Pappas, 'On the arithmetic moduli schemes of PEL Shimura varieties', J. Algebraic Geom. 9(3) (2000), 577-605.

[68] G. Pappas and M. Rapoport, 'Local models in the ramified case, II. Splitting models', Duke Math. J. 127(2) (2005), 193-250.

[69] G. Pappas and X. Zhu, 'Local models of Shimura varieties and a conjecture of Kottwitz', Invent. Math. 194 (2013), 147-254.

[70] V. Pilloni and B. Stroh, 'Cohomologie cohérente et représentations Galoisiennes', Ann. Math. Qué. 40(1) (2016), 167-202.

[71] R. Pink, 'Arithmetic compactification of mixed Shimura varieties', PhD Thesis, Rheinischen Friedrich-Wilhelms-Universität, Bonn, 1989.

[72] R. Pink, 'On $\ell$-adic sheaves on Shimura varieties and their higher direct images in the BailyBorel compactification', Math. Ann. 292 (1992), 197-240.

[73] R. Pink, T. Wedhorn and P. Ziegler, 'Algebraic zip data', Doc. Math. 16 (2011), 253-300.

[74] M. Rapoport and M. Richartz, 'On the classification and specialization of $F$-isocrystals with additional structure', Compos. Math. 103(2) (1996), 153-181.

[75] M. Rapoport and T. Zink, Period Spaces for p-divisible Groups, Annals of Mathematics Studies, 141 (Princeton University Press, Princeton, 1996).

[76] T. A. Springer, Linear Algebraic Groups, 2nd edn, Progress in Mathematics, 9 (Birkhäuser, Boston, 1998).

[77] H. Stamm, 'On the reduction of the Hilbert-Blumenthal-moduli scheme with $\Gamma_{0}(p)$-level structure', Forum Math. 9(4) (1997), 405-455. 
[78] B. Stroh, 'Compactification de variétés de Siegel aux places de mauvaise réduction', Bull. Soc. Math. France 138(2) (2010), 259-315.

[79] B. Stroh, 'Compactification minimale et mauvaise réduction', Ann. Inst. Fourier (Grenoble) 60(3) (2010), 1035-1055.

[80] B. Stroh, 'Sur une conjecture de Kottwitz au bord', Ann. Sci. Éc. Norm. Supér. (4) 45(1) (2012), 143-165.

[81] B. Stroh, 'Erratum à 'sur une conjecture de Kottwitz au bord”, Ann. Sci. Éc. Norm. Supér. (4) 46(6) (2013), 1023-1024.

[82] B. Stroh, Mauvaise réduction au bord, in Bost et al. [11], 269-304.

[83] E. Viehmann and T. Wedhorn, 'Ekedahl-Oort and Newton strata for Shimura varieties of PEL type', Math. Ann. 356(4) (2013), 1493-1550.

[84] T. Wedhorn, The Dimension of Oort Strata of Shimura Varieties of PEL-Type, in Faber et al. [18], 441-471.

[85] C. Zhang, 'Ekedahl-Oort strata for good reductions of Shimura varieties of Hodge type', Canad. J. Math. 70(2) (2018), 451-480. 